\title{
STRUCTURE BASED PREDICTIVE MODEL FOR COAL CHAR COMBUSTION
}

\section{FINAL TECHNICAL REPORT}

Principal authors:

Robert Hurt

Joseph Calo

Robert Essenhigh

Christopher Hadad

Report Issue Date: June 21, 2001

DOE Award Number: $\quad$ DE-FG22-96PC96249

Submitting Organization(s): Brown University (R. Hurt)

Division of Engineering, Box D

Providence, RI 02912

Ohio State University

Depts. of Mechanical Engineering

and Chemistry (Hadad).

206 W. 18th Avenue

Columbus, OH 43210-1107

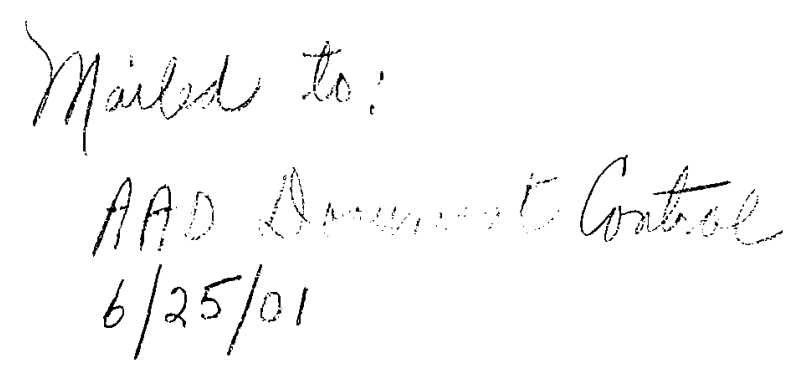




\section{Disclaimer}

This report was prepared as an account of work sponsored by an agency of the United States Government. Neither the United States Government nor any agency thereof, nor any of their employees, makes any warranty, express of implied, or assumes any legal liability or responsibility for the accuracy, completeness, or usefulness of any information, apparatus, product, or process disclosed, or represents that its use would not infringe privately owned rights. Reference herein to any specific commercial product, process, or service by trade name, trademark, manufacturer, or otherwise does not necessarily constitute or imply its endorsement, recommendation, or favoring by the United States Government or any agency thereof. The views and opinions of authors expressed herein do not necessarily state or reflect those of the United States Government or any agency thereof. 


\section{SELECTED TECHNICAL RESULTS}

\section{Chapter 1: Introduction, Overview of Results, Significance}

This unique collaborative project has taken a very fundamental look at the origin of structure, and combustion reactivity of coal chars. It was a combined experimental and theoretical effort involving three universities and collaborators from universities outside the U.S. and from U.S. National Laboratories and contract research companies. The project goal was to improve our understanding of char structure and behavior by examining the fundamental chemistry of its polyaromatic building blocks. The project team investigated the elementary oxidative attack on polyaromatic systems, and coupled with a study of the assembly processes that convert these polyaromatic clusters to mature carbon materials (or chars). We believe that the work done in this project has defined a powerful new science-based approach to the understanding of char behavior.

The work on aromatic oxidation pathways made extensive use of computational chemistry, and was led by Professor Christopher Hadad in the Department of Chemistry at Ohio State University. Laboratory experiments on char structure, properties, and combustion reactivity were carried out at both OSU and Brown, led by Principle Investigators Joseph Calo, Robert Essenhigh, and Robert Hurt. Modeling activities were divided into two parts: first unique models of crystal structure development were formulated by the tream at Brown (PI's Hurt and Calo) with input from Boston University and significant collaboration with Dr. Alan Kerstein at Sandia and with Dr. Zhong-Ying chen at SAIC. Secondly, new combustion models were developed and tested, led by Professor Essenhigh at OSU, Dieter Foertsch (a collaborator at the University of Stuttgart), and Professor Hurt at Brown. One product of this work is the CBK8 model of carbon burnout, which has already found practical use in CFD codes and in other numerical models of pulverized fuel combustion processes, such as EPRI's NOxLOI Predictor.

The remainder of the report consists of detailed technical discussion organized into chapters whose organization is dictated by the nature of the research performed. Chapter 2 is entitled "Experimental Work on Char Structure, Properties, and Reactivity," and focuses on fundamental structural studies at Brown using both phenol/formaldehyde resin chars as model carbons and real coal chars. This work includes the first known in site high resolution TEM studies of carbonization processes, and some intriguing work on "memory loss," a form of interaction between annealing and oxidation phenomena in chars.

Chapter 3 entitled "Computational Chemistry of Aromatic Oxidation Pathways" presents in detail the OSU work targeted at understanding the elementary molecular pathways of aromatic oxidation. Chapter 4 describes the "Mesoscale Structural Models," using a combination of thermodynamic (equilibrium) approaches based on liquid crystal theory and kinetic simulations accounting for the effects of limited layer mobility in many fossil fuel derived carbons containing cross-linking agents. Chapter 5 entitled "Combustion Modeling" presents work on extinction in the late stages of combustion and the development and features of the CBK8 model.

The project has given rise to a large number of scientific publications and presentations, with contributions from each of the main project tasks. Selected publications and presentations from 
Hurt, R.H., "Designing Carbon Materials by Polyaromatic Self Assembly" presented at the Department of Chemical Engineering, The Technical University of Denmark, April, 2001.

Hurt, R.H., "Self Assembled Nanostructures in Carbon Materials" presented at the Department of Energy and Geoenvironmental Engineering, The Pennsylvania State University, February, 2001.

Shim, H-S., Hurt, R.H., Crawford, G., Woodward, C., and Bernstein, S. "Investigation of Surface Anchoring in Mesophase Pitch by In Situ and Ex Situ Optical Microscopy" Proceedings of Eurocarbon 2000: The First World Conference on Carbon, Berlin, July, 2000.

Sun, J.K., Hurt, R.H., "Mechanisms of Extinction and Near-Extinction in Pulverized Solid Fuel Combustion," Proceedings of the Combustion Institute, Vol. 28, The Combustion Institute, 2000, pp. 2205-221. 


\section{Chapter 2: Experimental Work on Char Structure, Properties, and Reactivity}

In this task, experimental work was conducted in support of the development of new models that describe coal char structure during combustion on a more fundamental basis. This work was focused on laboratory-scale experiments designed to establish link between char structure and oxidation reactivity. The specific subtasks included:

- Char Structure via HRTEM Fringe Imaging

- The effect of char "age"

- Hot-stage fringe imaging

- The effects of mineral matter

- Char Reactivity

- Model and coal chars

- The effect of char burn-off on reactivity

- Char Porosity Characterization

- Using small angle scattering and gas adsorption

- As a function of burn-off

- Mechanisms of Nanoscale Order Development in Carbons

- Interfacial ordering by liquid crystal surface anchoring.

\subsection{The Development of Carbon Structure From HRTEM Fringe Imaging.}

The objectives of the HRTEM experiments were to monitor structure development during ordering and graphitization,, in order to improve the understanding of these processes, identify mechanisms, and provide data for model development and validation.

Phenolic resin char samples of with different heat treatment histories (i.e., char "age") were investigated at ambient temperature. In addition, real-time, hot-stage HRTEM fringe imaging experiments were attempted.

2.1-1. Preparation of Phenol Resin Char (PRC) Model Compound. The phenolic resin char (PRC) model compound was prepared from resin synthesized in our laboratory. ${ }^{1}$ Phenol and formaldehyde are mixed in a molar ratio of 1:4.33. Ammonium hydroxide is used as a catalyst, rather than sodium hydroxide in order to minimize the final metals content. The resultant mixture is heated in a flask attached to a reflux condenser in a hot water bath until condensation occurs. The sample is then cooled down in an argon atmosphere and left to settle for 24h or until two distinct phases are clearly present. The two phases are separated, and the resin is neutralized with glacial acetic acid and washed with distilled water. The resultant material is then dissolved in acetone. The resin material is then cured in vacuo, first for $2 \mathrm{~h} @ 60^{\circ} \mathrm{C}$, and then $12 \mathrm{~h} @ 120^{\circ} \mathrm{C}$, and finally in atmospheric pressure helium for $2 \mathrm{~h} @ 300^{\circ} \mathrm{C}$. The overall reaction for the formation of the phenolformaldehyde resin is: 

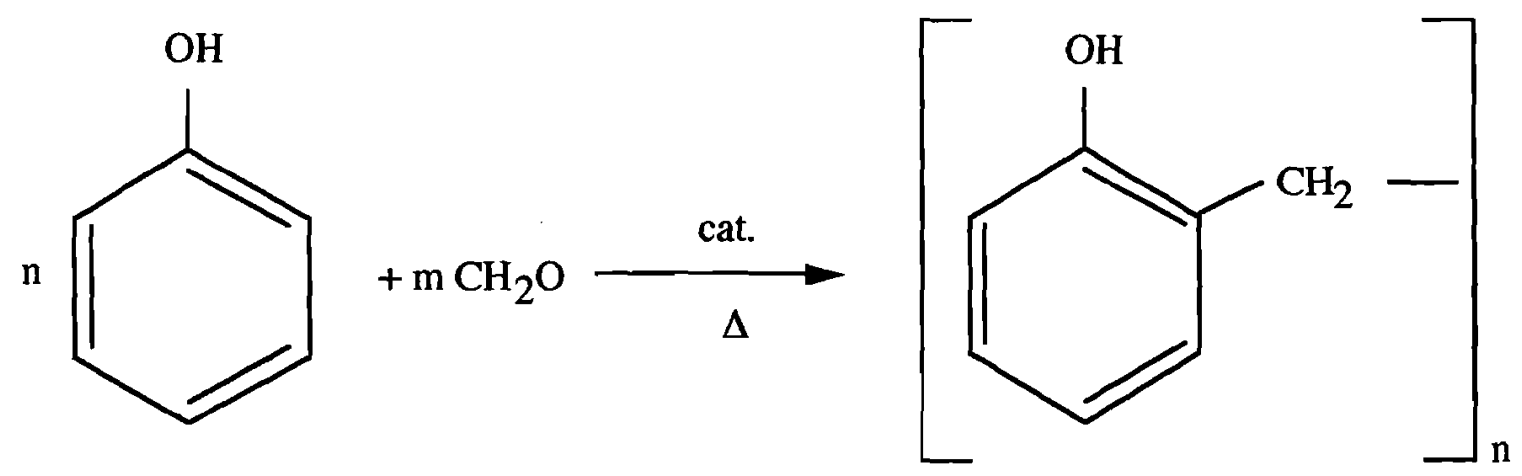

Char is then prepared from the resin by pyrolysis in flowing helium. Typical resultant elemental compositions for PRCs produced at four different pyrolysis temperatures for one hour are presented below in Table 2.1-1.

Table 2.1-1. Elemental composition of phenolic resin char.

\begin{tabular}{|r|r|r|r|r|r|r|r|}
\hline $\mathrm{T}_{\mathrm{p}}\left[{ }^{\circ} \mathrm{C}\right]$ & \multicolumn{4}{|c|}{ Elemental Composition (wt \%) } & \multicolumn{3}{|c|}{ Atomic Ratio x 100 } \\
\hline & $\mathrm{N}$ & \multicolumn{1}{c|}{$\mathrm{C}$} & $\mathrm{H}$ & \multicolumn{1}{c|}{$\mathrm{O}$} & \multicolumn{1}{c|}{$\mathrm{O} / \mathrm{C}$} & \multicolumn{1}{c|}{$\mathrm{N} / \mathrm{C}$} & \multicolumn{1}{c|}{$\mathrm{H} / \mathrm{C}$} \\
\hline 1000 & 0.4 & 97.1 & 0.8 & 1.7 & 1.3 & 0.4 & 9.9 \\
\hline 1111 & 0.2 & 97.9 & 0.7 & 1.2 & 0.9 & 0.2 & 8.6 \\
\hline 1243 & 0.2 & 98.5 & 0.3 & 1.0 & 0.8 & 0.2 & 3.7 \\
\hline 1400 & 0.1 & 98.8 & 0.3 & 0.8 & 0.6 & 0.1 & 3.6 \\
\hline
\end{tabular}

A schematic of an average aromatic cluster constructed from the measured elemental composition presented in Table 2-1 is given below in Figure 2.1-1.

2.1-2. Experimental Procedures. Phenol-formaldehyde resin was ground and sieved into 250 $149 \mu \mathrm{m}$ and $149-75 \mu \mathrm{m}$ size ranges. Two char samples that differed significantly in "age" were prepared via pyrolysis at $900^{\circ} \mathrm{C}$ for $1 \mathrm{~h}$, and at $1300^{\circ} \mathrm{C}$ for $4 \mathrm{~h}$ in helium. Oxygen $\left(500^{\circ} \mathrm{C}\right) \mathrm{TGA}$ reactivities of these two samples differed by over an order of magnitude. This difference is due almost entirely to structure differences between the two samples.

For the hot-stage experiments, the samples were set on molybdenum grids with an amorphous carbon coating. The sample-loaded grid was placed in the HRTEM and left to degas overnight. An experiment was initiated by examining the sample at ambient temperature. The temperature of the sample was then raised to about $800^{\circ} \mathrm{C}$, while continuously recording images on videotape. This procedure was then repeated at $100^{\circ} \mathrm{C}$ temperature intervals up to a maximum temperature of $1200^{\circ} \mathrm{C}$. 


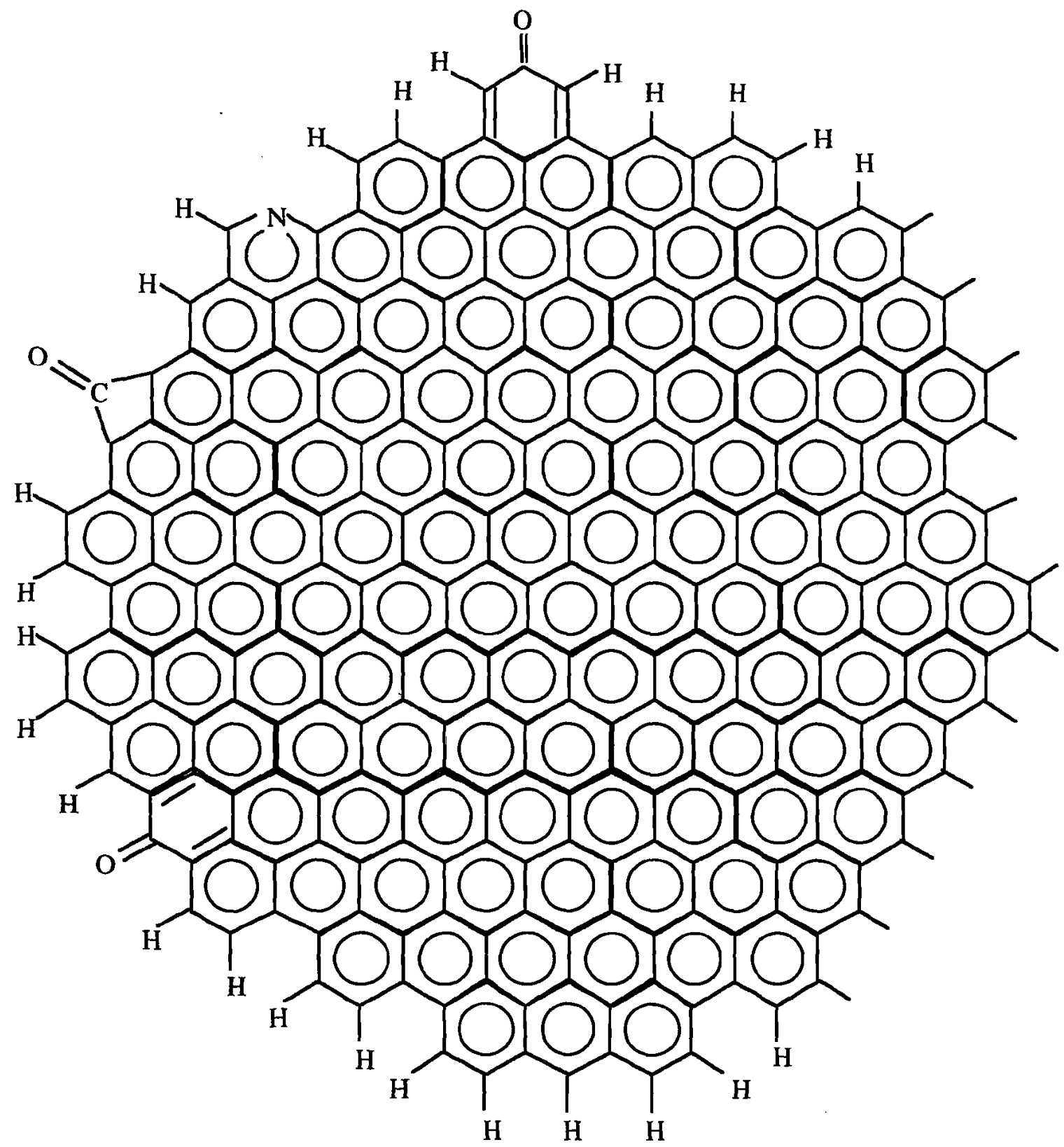

Figure 2.1-1. Schematic of average aromatic cluster for $1000^{\circ} \mathrm{C} \mathrm{PRC}$, as deduced from elemental composition.

2.1-2. Results. HRTEM micrographs of the $900^{\circ} \mathrm{C}$ and $1300^{\circ} \mathrm{C}$ char samples are presented in Figures 2.1-2 and 2.1-3, respectively. Presented along with each of the images are the corresponding digitally-processed images according to the procedure of Shim and Hurt ${ }^{2}$. As clearly shown, the $900^{\circ} \mathrm{C}$ char sample is much more disordered than the $1300^{\circ} \mathrm{C}$ char sample. This is reflected in the correlation coefficient, which is much less for the former than for the latter sample. Therefore, it is concluded that the ordering of PRC occurs to a considerable degree over the temperature range accessible with the hot-stage of the HRTEM. 
The results of these experiments suggested that the hot-stage approach may be useful for observing and identifying growth mechanisms of ordered carbon structures, particularly in "real time."

The initial experiments with real-time, hot-stage HRTEM were conducted with samples of unactivated, $900^{\circ} \mathrm{C}$ PRC (i.e., $0 \%$ burn-off), and the same material burned-off to 5.4\%. The videotaped images were of particularly good quality for the first ( $0 \%$ burn-off) experiment. One frame from this experiment is presented as Figure $2.1-4$ at $1177^{\circ} \mathrm{C}$ where a band of ten or so ordered graphitic planes appeared to grow from an interface between two adjacent regions. For the most part, however, the hot-stage approach proved to be difficult experimentally and did not yield very useful information. To be sure, fringe image micrographs are certainly useful for quantitative analysis of final ordered structures following thermal annealing. However, capturing the ordering process at temperature and in real time was quite difficult. This was attributed to several factors. One important experimental issue was that the quality of the $\mathrm{Mo}(\mathrm{C})$ grids that were used varied significantly from grid-to-grid. Since fringe imaging is difficult to perform even with a stable grid, when it becomes unstable at high temperatures, the resultant images tend to be inconclusive at best. Another recurring problem was that of impurities which tended to appear at higher temperatures. These may be related to metals from the grid and/or from the hot-stage apparatus. This point is related to the potential effects of mineral matter impurities in ordering carbon structure, which may be important in coal chars at combustion temperatures. However, ultimately it was concluded that despite the experimental difficulties, it is inherently difficult to capture the ordering process on the requisite time scales because of the nature of the wide distribution of activation energies for the many processes responsible for ordering. The processes with very low activation energies occur more rapidly than the time scale over which the temperature can be varied and stabilized; and the processes with very large activation energies occur on very long time scales that are impractical to observe with respect to the requisite microscope time and long-term sample and temperature stability. For the reasons, this approach was not pursued further for the intended purpose.

\subsection{The Effects of Mineral Matter on Carbon Structure.}

An important issue in developing models of structure development in coal chars is the role of mineral matter impurities. It has often been suspected and there is some evidence that the presence of mineral matter may affect the structure and ordering of carbon. ${ }^{3,4}$ Lunden et al. ${ }^{5}$ observed a considerable amount of inorganic mineral matter in "solid solution" with the carbonaceous matrix from Pittsburgh \#8 (PSOC 1451) samples obtained from a laminar flow, flame-supported entrained flow reactor. It was hypothesized in this work that the intimate mixing of carbonaceous and inorganic constituents may affect char reactivity by both blocking oxygen access to active carbon sites and influencing microscopic carbon structure that evolves during combustion.

A common approach used to explore the effects of mineral matter impurities on reactivity has been to demineralize coals and chars by washing in strong acids (e.g., see Morgan and Jenkins ${ }^{6}$ ). One of the drawbacks of this technique is that it almost certainly causes significant alterations of the structure of the resultant material. Consequently, an "inverse" approach was used here; that is doping of a "pure" carbon with mineral matter. The carbon material was derived from a high carbon content fly ash (Fly Ash 23 from the Salem Harbor Power Plant. 

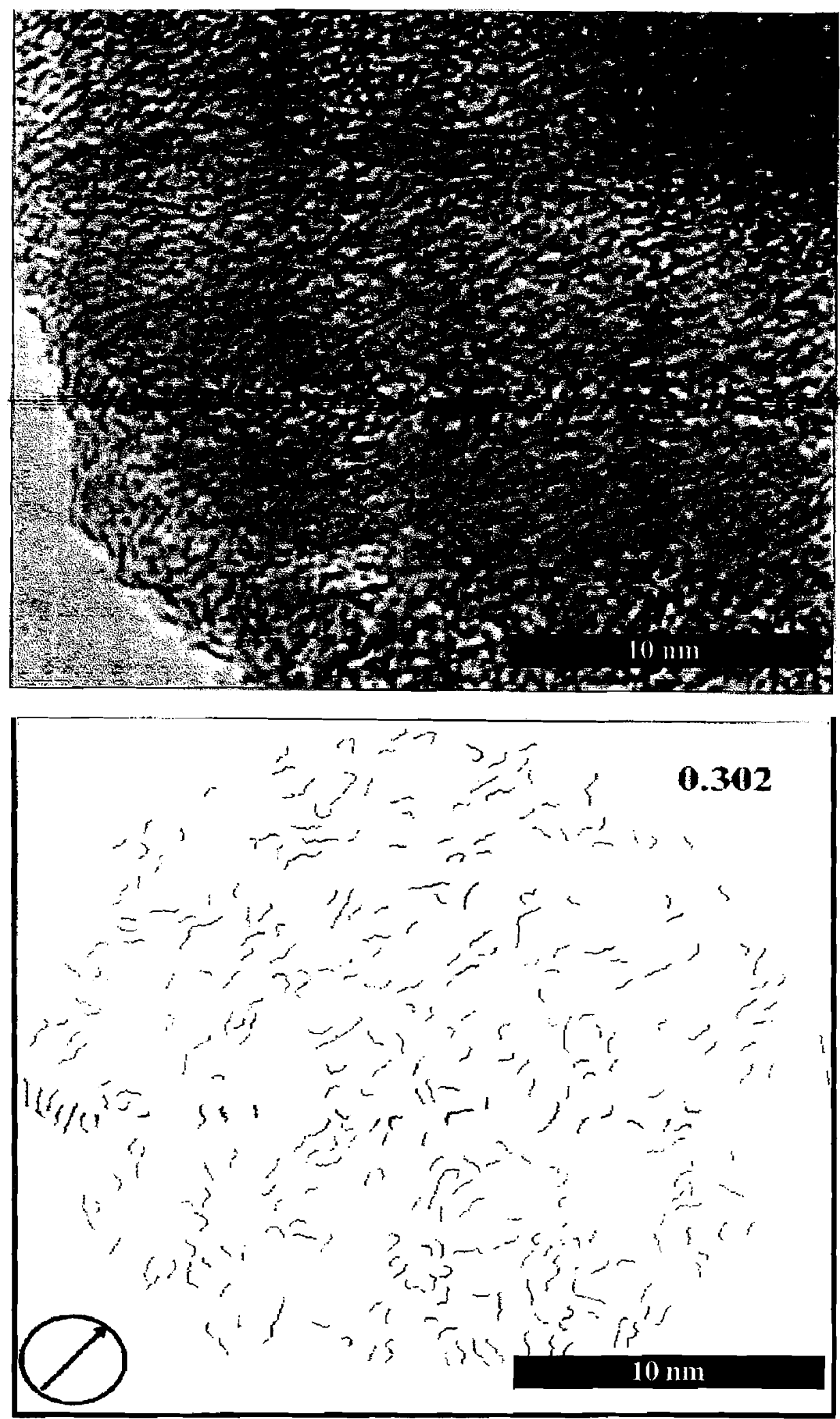

Figure 2.1-2. HRTEM fringe image of $900^{\circ} \mathrm{C}$ resin char, and the corresponding digitallyprocessed image (Shim and Hurt, 1997). 

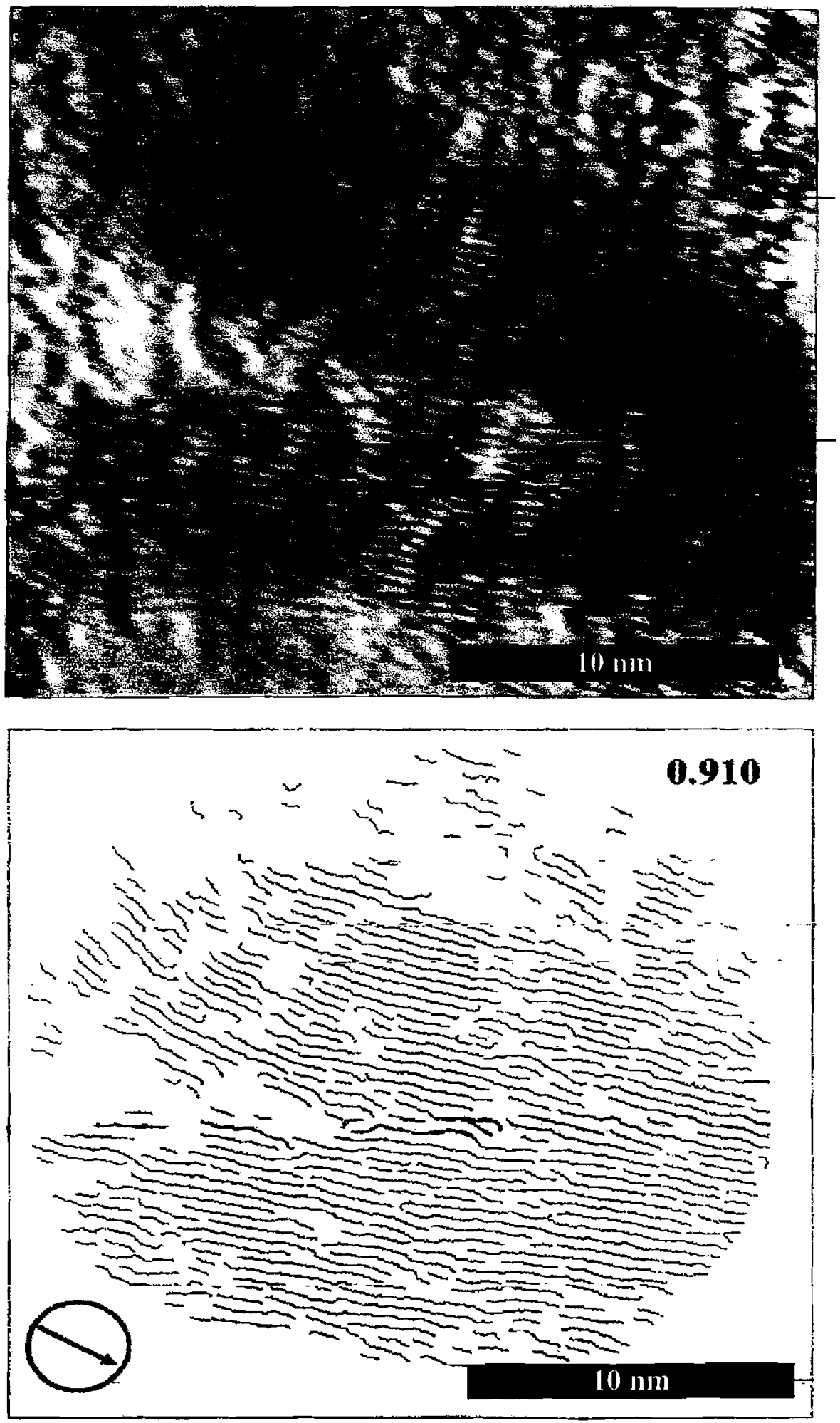

Figure 2.1-3. HRTEM fringe image of $1300^{\circ} \mathrm{C}$ resin char, and the corresponding digitallyprocessed (Shim and Hurt, 1997). 


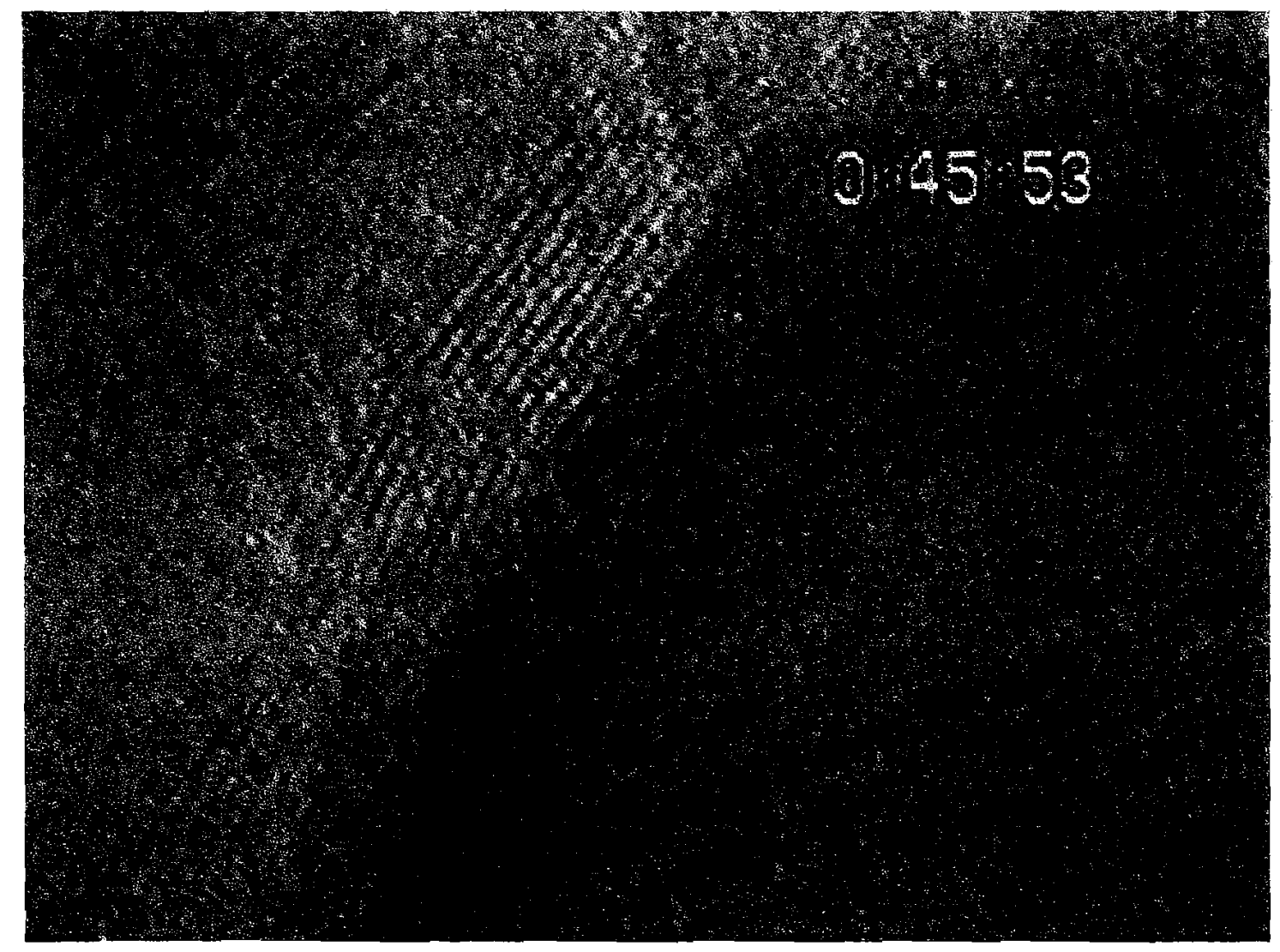

Figure 2.1-4. Frame from HRTEM fringe imaging videotape of ordering of $900^{\circ} \mathrm{C}(1 \mathrm{~h}$ in helium; $0 \%$ burn-off) phenol-formaldehyde resin char at $1177^{\circ} \mathrm{C}$.

The first step in the preparation of the samples was to increase the carbon content in the fly ash. This was done by sieving the fly ash while it was being shaken mechanically. In this manner it was possible to collect an optimal particle size for use in the high temperature flame reactor for reactivity measurements, while at the same time comminution of the sample due to shaking tended to loosen fine ash particles, resulting in a sample that was more enriched in carbon. The resultant sieved material was found to be $84 \%$ carbon. The mineral matter content of $16 \%$ is comparable to that found in typical bituminous coal chars.

The ash was obtained from Pittsburgh \#8 coal (PSOC 1451). The coal was first pyrolyzed up $700^{\circ} \mathrm{C}$ until gas evolution was observed to cease. Following cooldown, it was crushed and then ashed in pure oxygen at $500^{\circ} \mathrm{C}$. The resultant particle size fraction $<40 \mu \mathrm{m}$ was used to dope the carbon samples.

SEM micrographs of the ash material and the carbon are presented in Figures 2.2-1 and 2.2-2, respectively. As shown, the ash particles are sufficiency small to fit in the void spaces available in the carbon. 


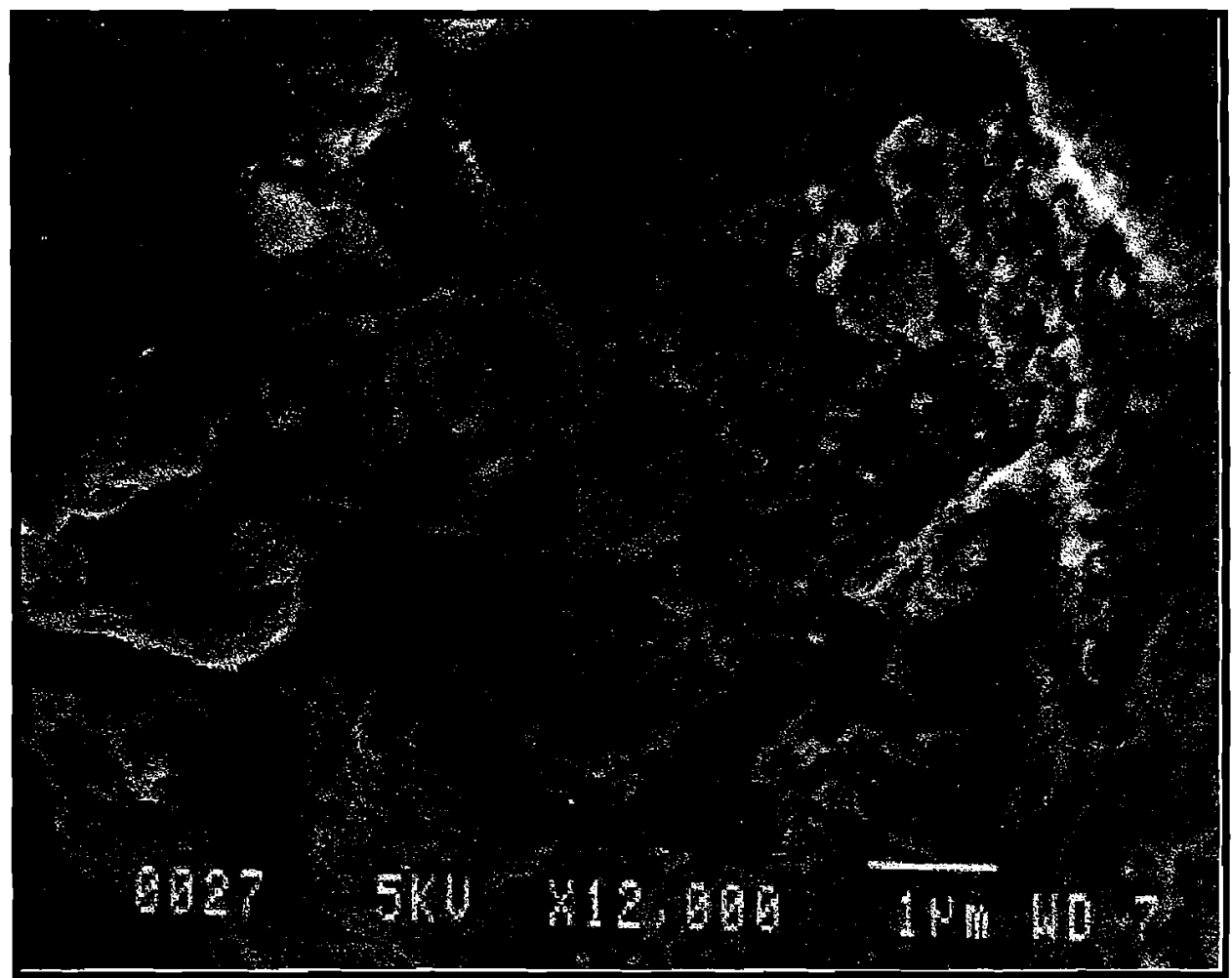

Figure 2.2-1. Ash from Pittsburgh \#8 coal. Pyrolyzed at $700^{\circ} \mathrm{C}$ in a tube furnace, and ashed at $500^{\circ} \mathrm{C}$ in $\mathrm{O}_{2}$.

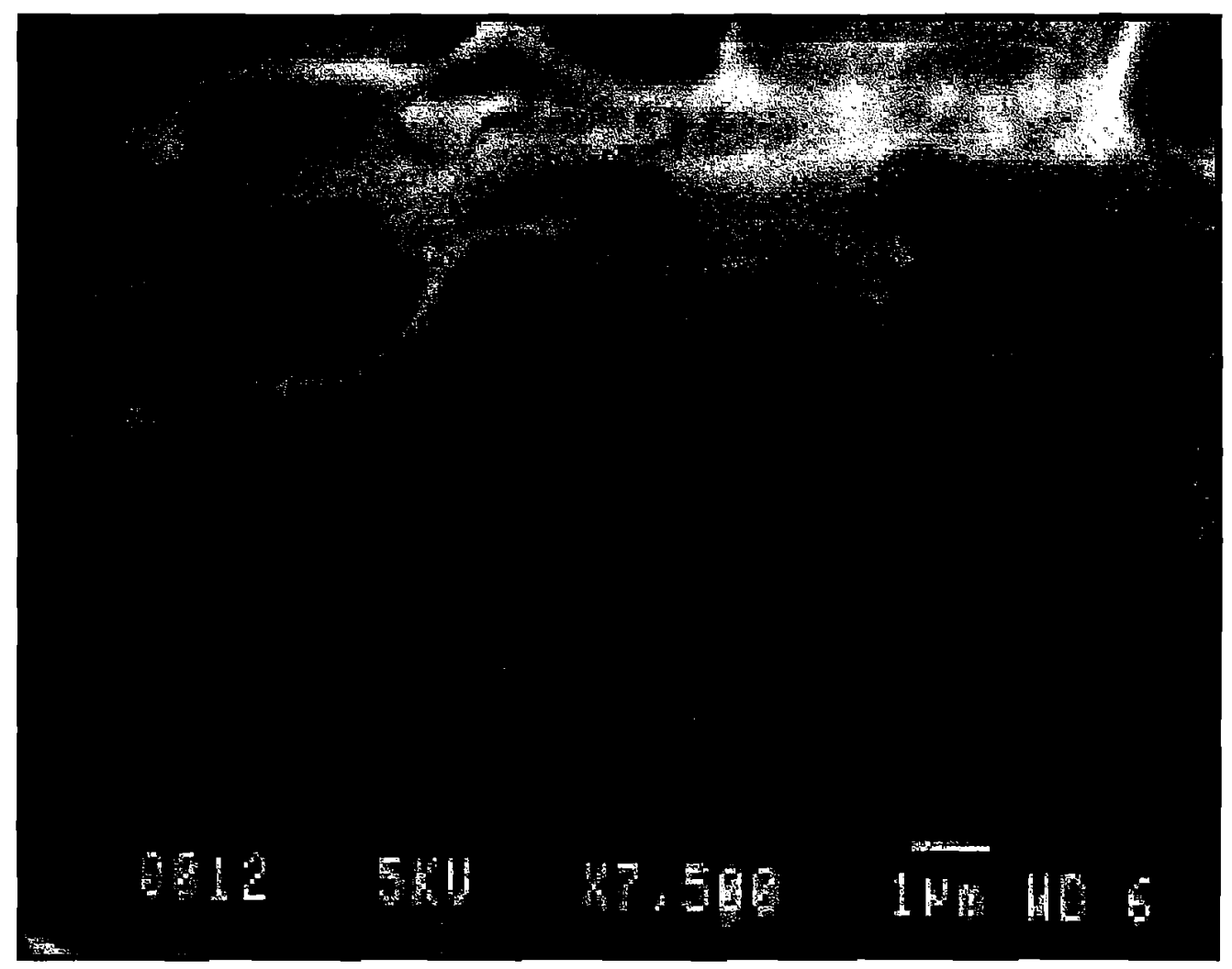




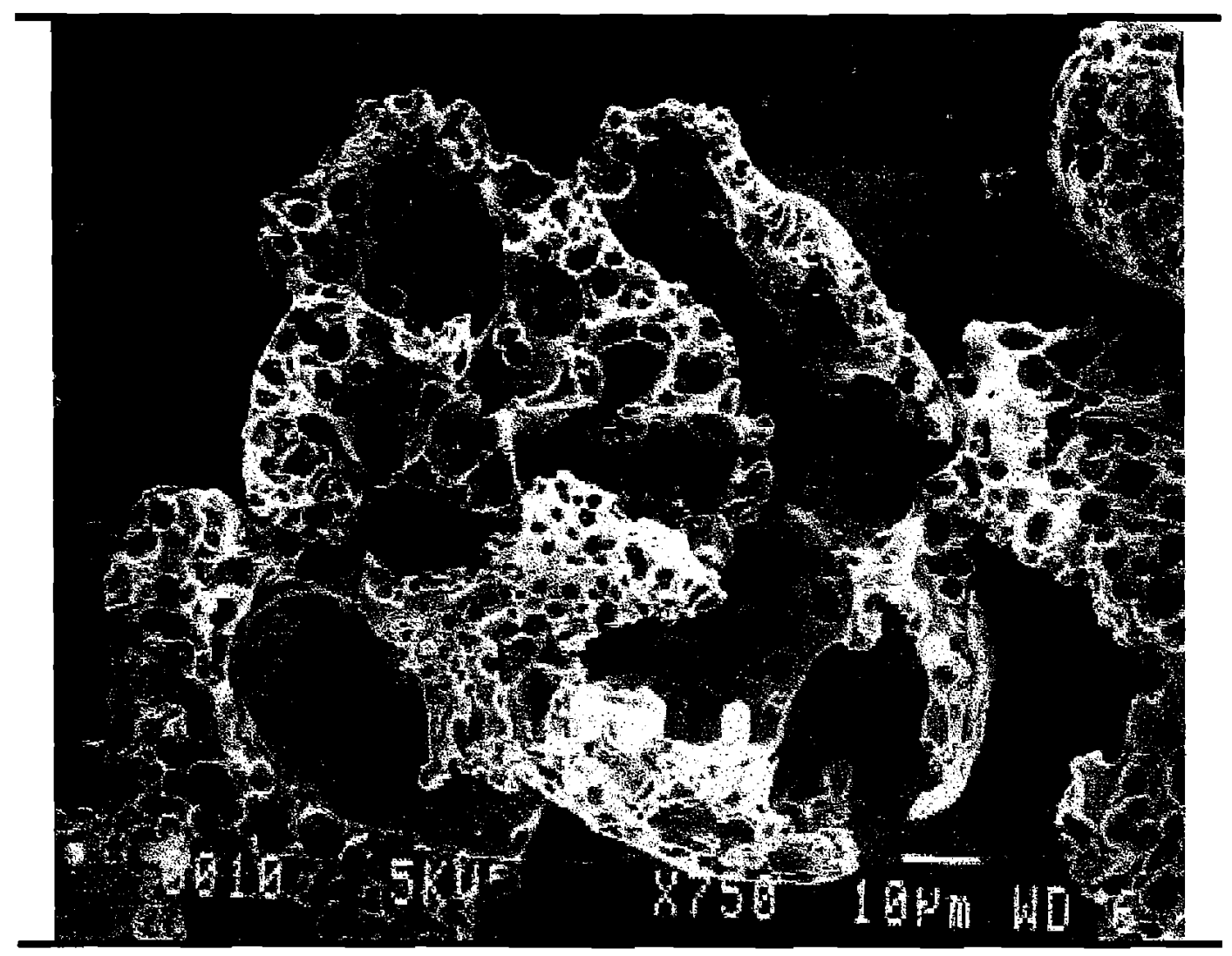

Figure 2.2-2. SEM micrographs of Salem Harbor residual carbon (Fly Ash 23; 16\% Ash).

The final doped samples were dried at $383 \mathrm{~K}$ for $2 \mathrm{~h}$, and then burned in a high temperature flame reactor fitted with rapid quench extractive sampling. ${ }^{7}$ The reactor was fed by a Hencken burner fueled with a $\mathrm{CH}_{4} / \mathrm{O}_{2} / \mathrm{Ar}$ mixture, producing a post-flame oxygen concentration of $14 \mathrm{~mol} \%$. The mean residence time was $140 \mathrm{~ms}$, and the centerline temperature was $1280 \mathrm{~K}$. Particle temperatures were estimated to range from $1650-1800 \mathrm{~K}$. The sample feed rate was less than $1 \mathrm{~g} / \mathrm{h}$. Additional details of the apparatus are described elsewhere. ${ }^{6} 4$ schematic of the apparatus is presented in Figure 2.2-3.

The average reaction rate on a carbon basis in the flame reactor was determined by dividing the mass loss by the initial carbon content and the mean residence time. In Figure 2.2-4 are presented values of this average reaction rate for the doped samples as a function of initial ash content. As shown, the specific reaction rate decreases with increasing ash content by about an order of magnitude over the ash content range investigated. It is also interesting to note that the shape of this curve is reminiscent of isothermal TGA reactivity curves for Pittsburgh \#8 coal char (pyrolyzed at $1573 \mathrm{~K}$ for $4 \mathrm{~h}$ ) in oxygen at lower temperatures.

The SEM micrographs for the undoped sample ( $8 \%$ total ash content), before and after the flame reactor are presented in Figures 2.2-5 (a) and (b), respectively. From these figures, it is apparent that the mean carbon particle size was reduced due to reaction, while the ash particles appear to 
have agglomerated and coalesced, creating larger particles of about 2-3 $\mu \mathrm{m}$ in size. A closer examination reveals that the carbon has been reacted away, creating more irregularly shaped openings and a rougher texture than for the previously smooth carbon surfaces.

In Figures 2.2-6 (a) and (b) are presented the corresponding SEM micrographs for the doped sample (68\% total ash content). The micrograph for the freshly prepared sample shows that the added ash particles effectively cover the surfaces of the carbon particles. The added ash can be distinguished from the smooth, pearl-like ash particles original to the fly ash by their irregular shape. The micrograph in Figure 2.2-6 (b), taken after reaction, shows not only an increase in the relative amount of ash, but that some of the ash has fused and covered carbon particles. This latter mechanism may be the primary reason for the observed decrease in specific reaction rate with increasing ash content; i.e., an increasing amount of carbon becomes inaccessible to oxygen by being covered with a fused, "protective," ash layer. 


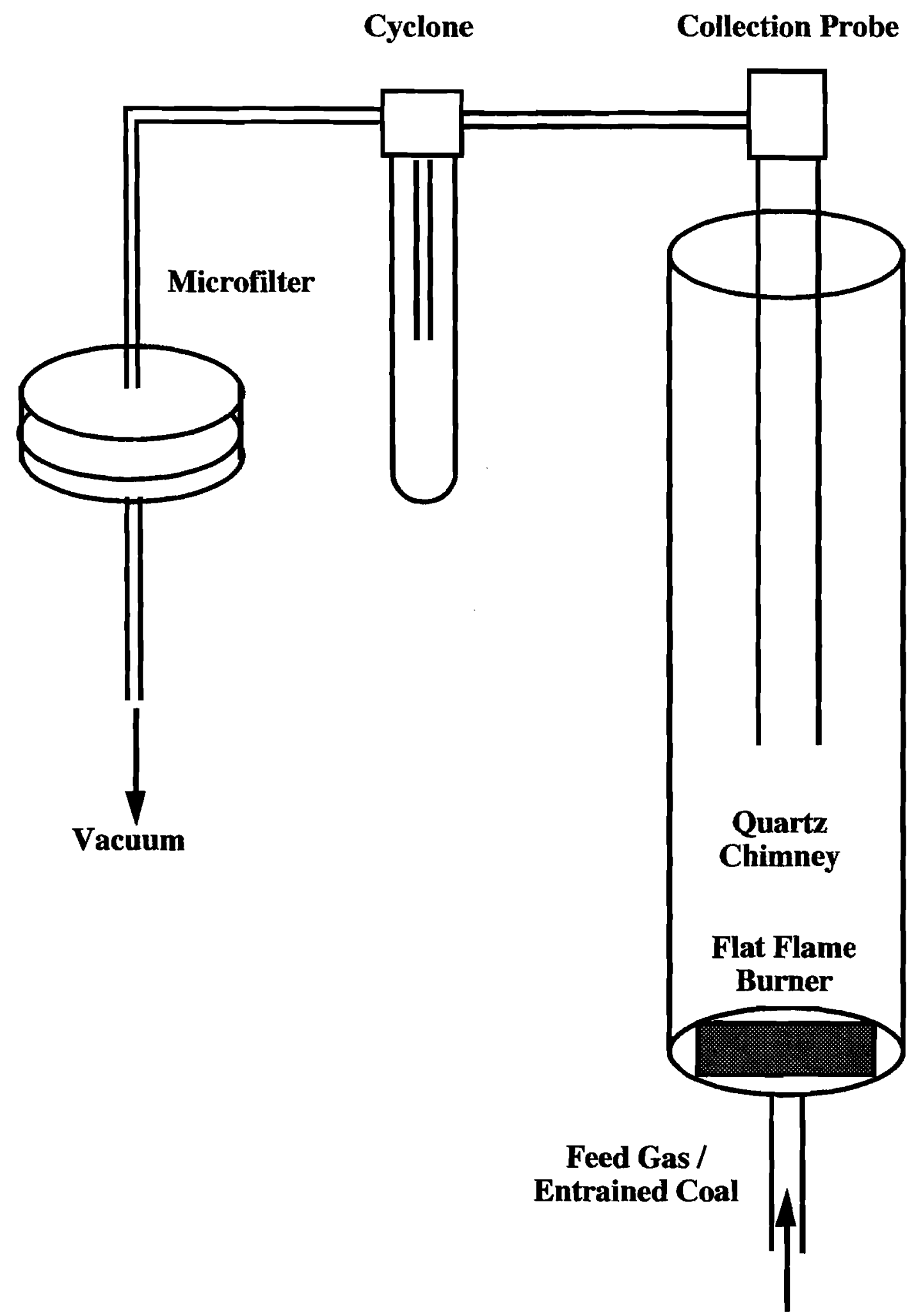

Figure 2.2-3. Schematic of Hencken burner/reactor system. 


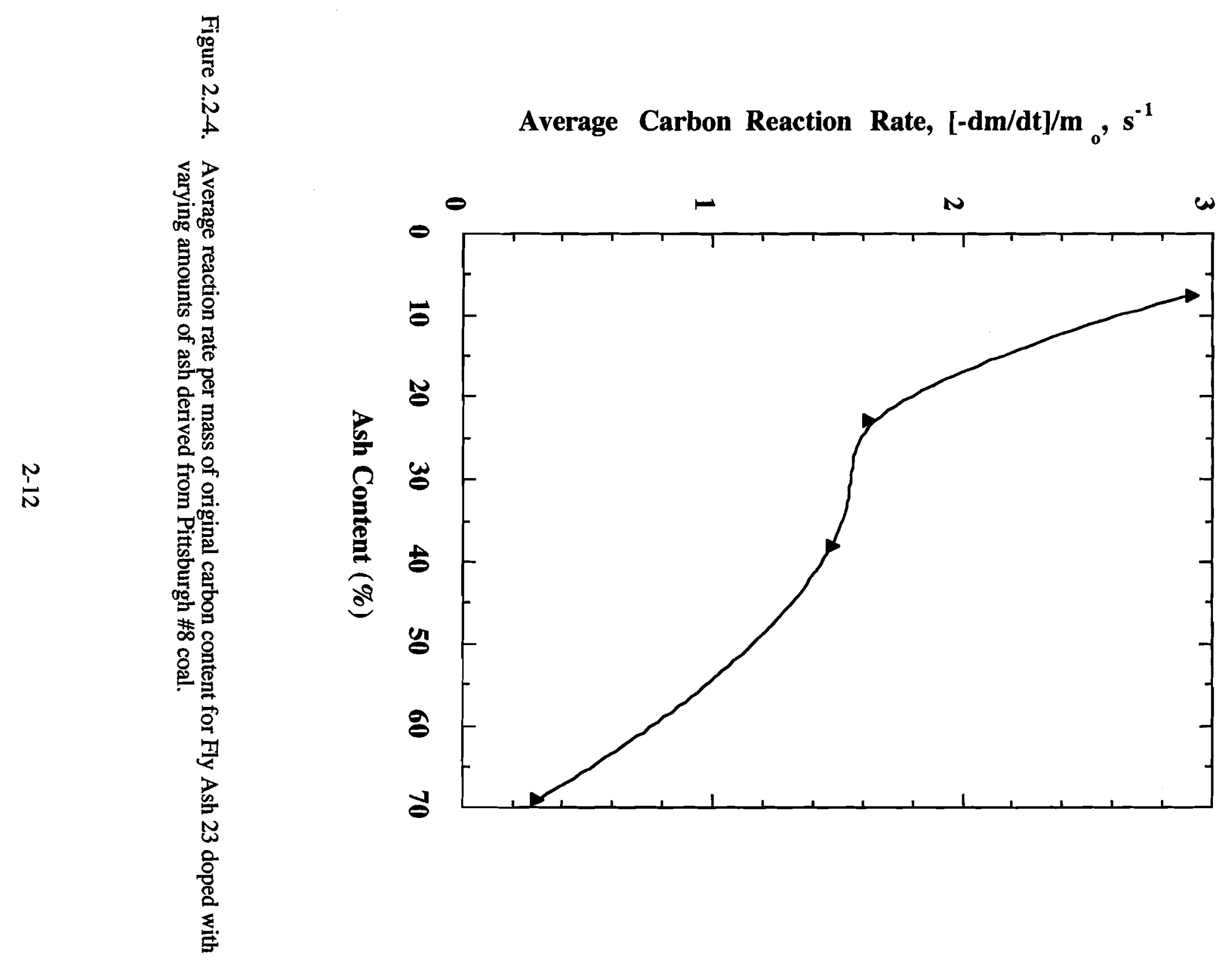




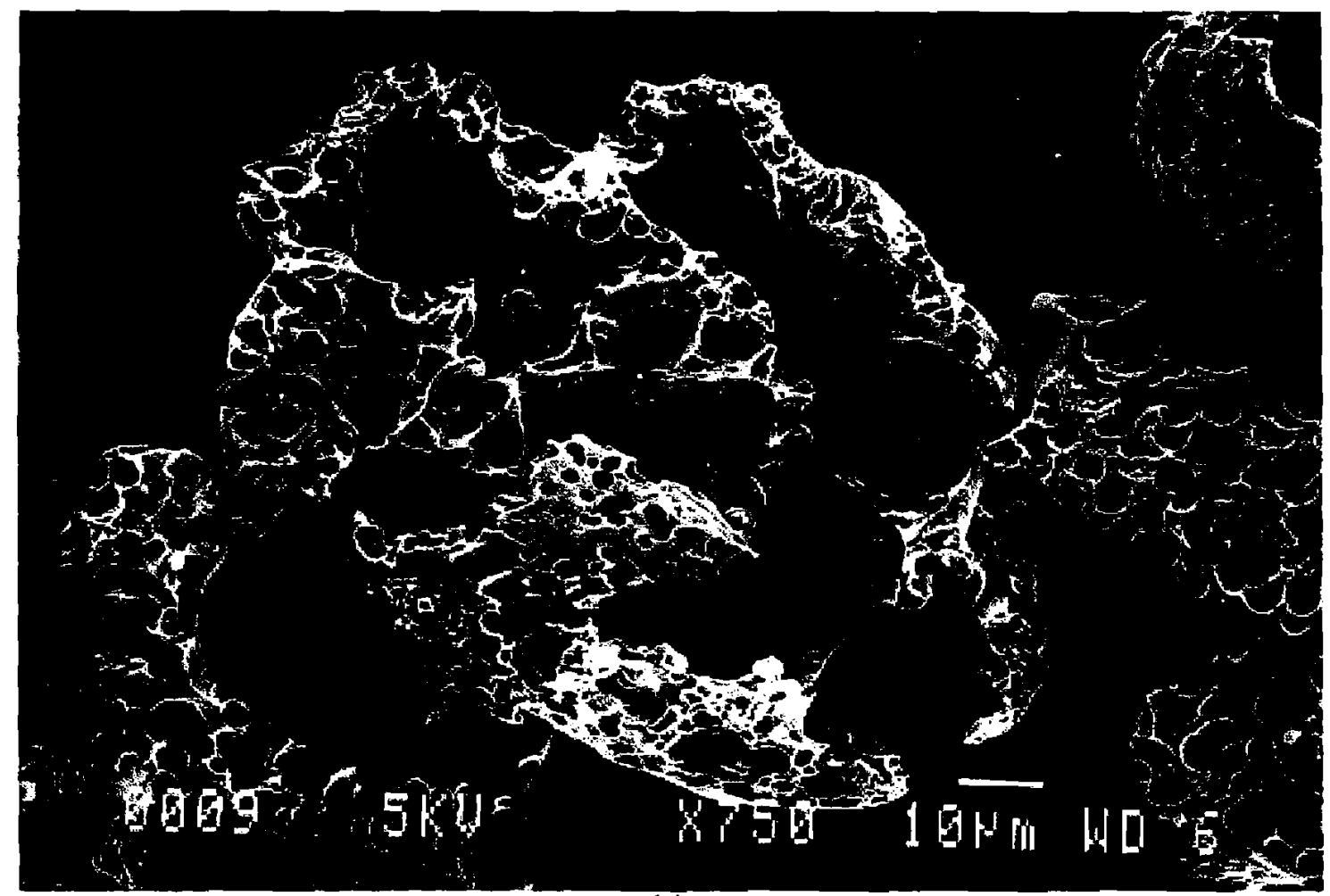

(a)

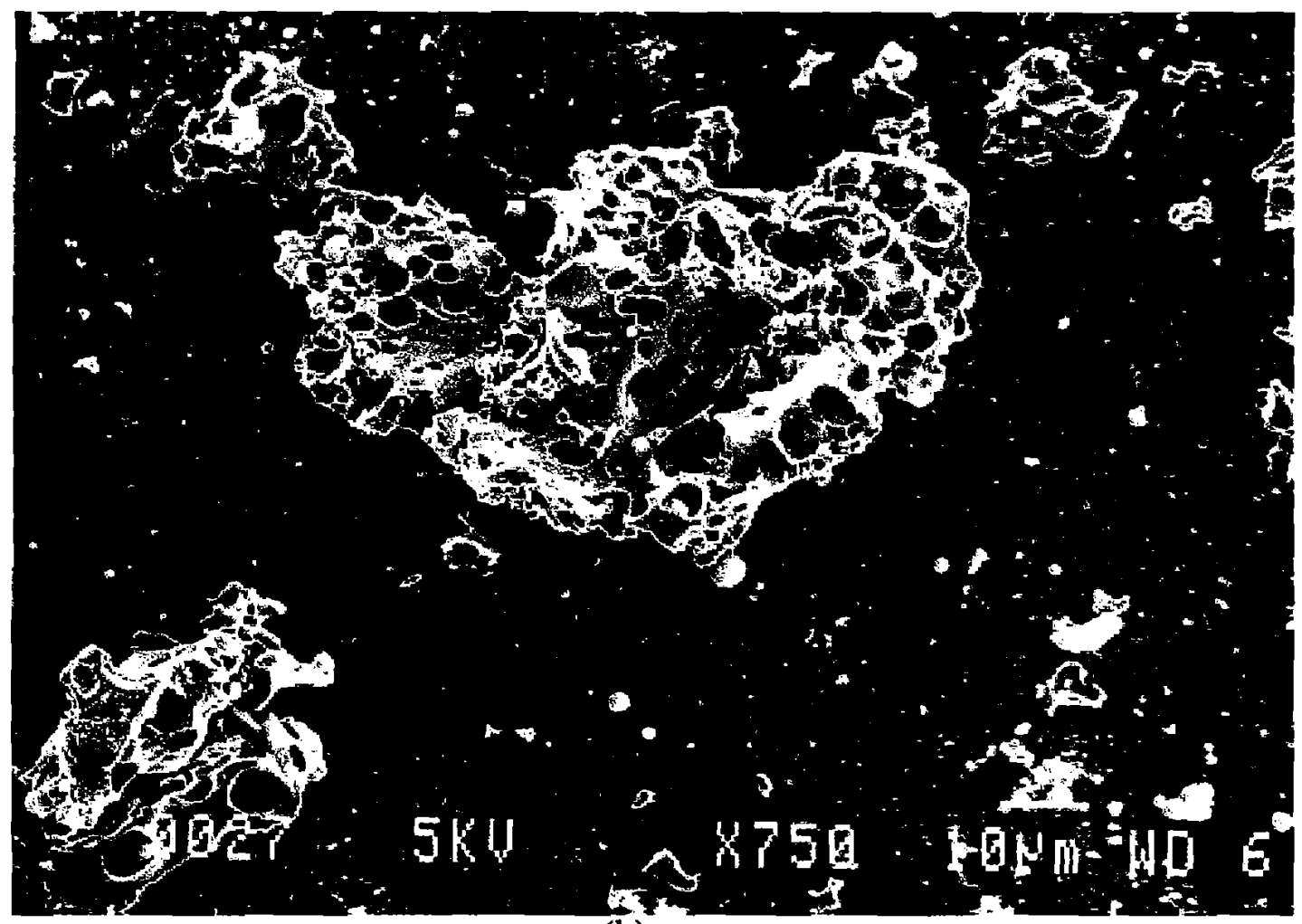

(b)

Figure 2.2-5. SEM micrographs of undoped sample FA1.1 (8\% ash), before (a) and after (b) reaction. 


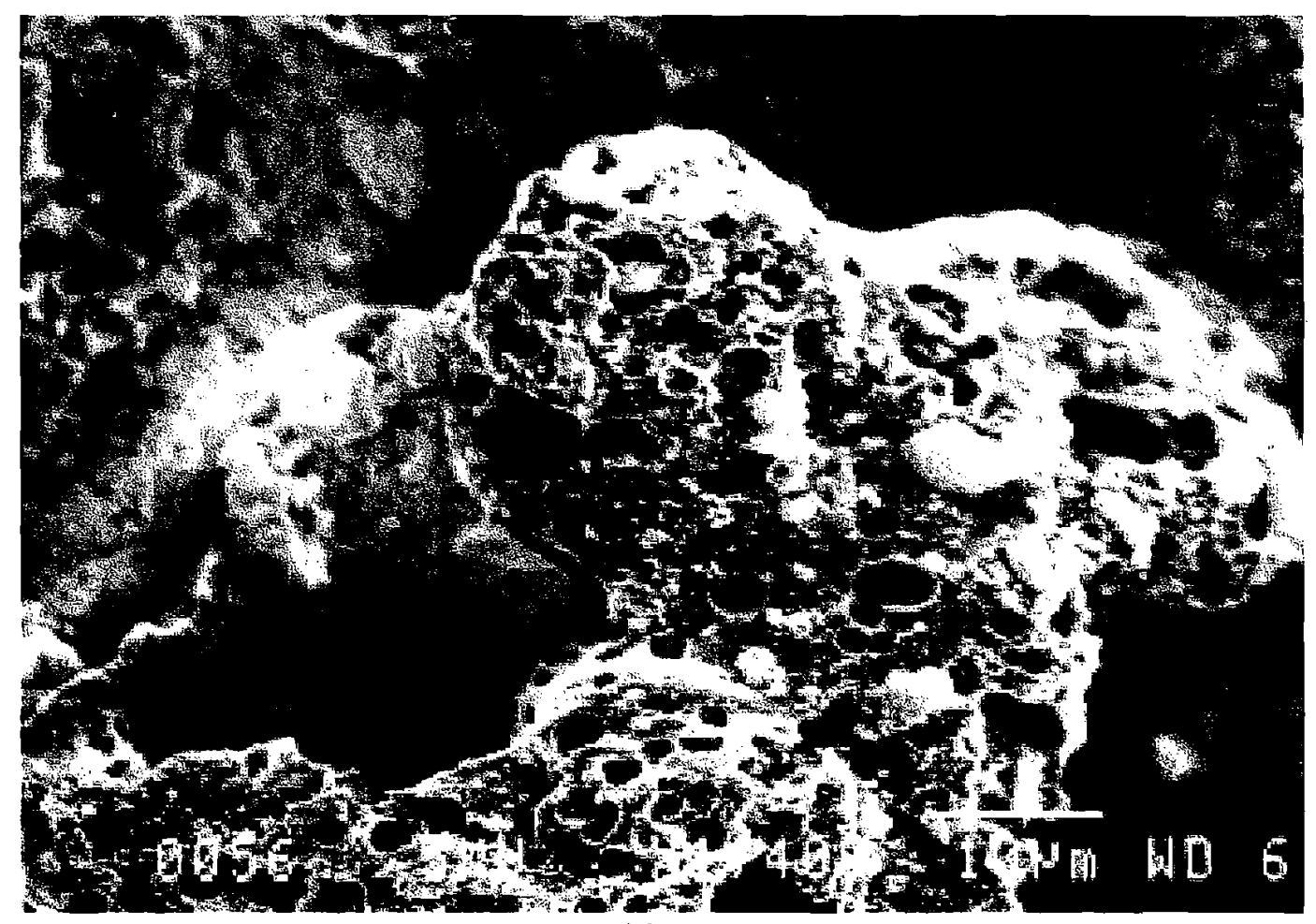

(a)

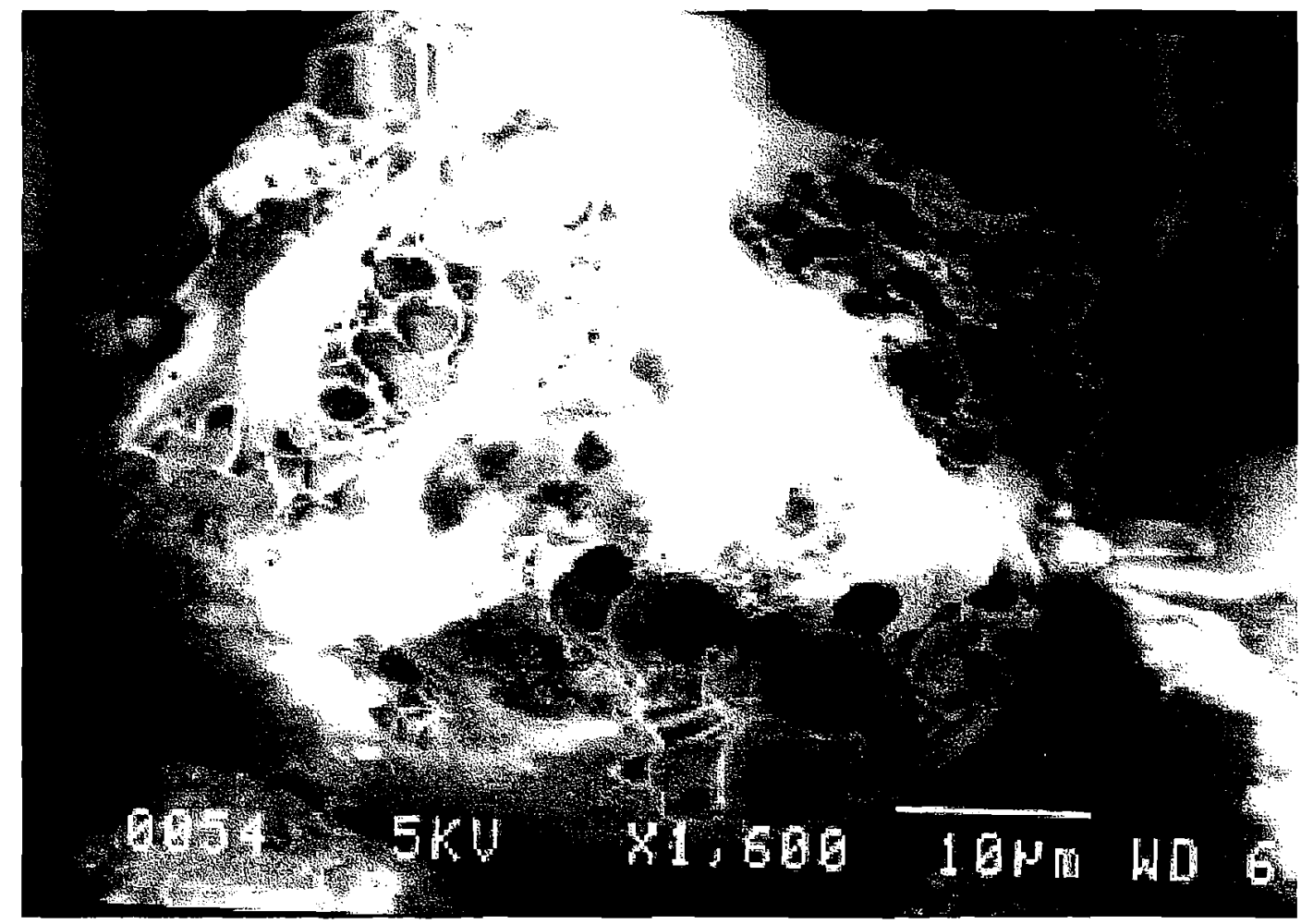

(b)

Figure 2.2-6. SEM micrographs of sample FA1.4 (68\% total ash) before (c) and after (d) reaction. 


\subsection{The Reactivity Behavior of ${ }^{13}$ C-Labeled Phenol-Formaldehyde Resin Char.}

A number of studies have examined the dependence of the morphology of phenol-formaldehyde resin chars (PRC) on pyrolysis conditions. ${ }^{8,9,10,11,12}$ The objective of the current work is to examine the manner in which gasification occurs in these materials as a function of pyrolysis regimen, char burn-off, and char structure. To this end, a phenol formaldehyde resin was synthesized using ${ }^{13} \mathrm{C}$-labeled formaldehyde. Since the labeled carbons are concentrated in the methylene bridges in the polymer, and there is evidence that these decompose first during pyrolysis, it is hypothesized that a larger proportion of the labeled carbon would reside in the amorphous carbon material than in the aromatic rings which are primarily formed from the unlabeled phenol. The evolution of labeled and unlabeled carbon as $\mathrm{CO}$ and $\mathrm{CO}_{2}$ was monitored during gasification and post-gasification desorption experiments in order to investigate this hypothesis.

2.3-1. Experimental. The synthesis procedure for the resin is as described above. ${ }^{1}$ Two char samples, Sample 1 and Sample 2, were prepared at $850^{\circ} \mathrm{C}$ and $1000^{\circ} \mathrm{C}$ for 1.5 and 3 hours, respectively, in a helium atmosphere. The particles were 30-100 Tyler mesh. The samples were heated in a TGA system to $850^{\circ} \mathrm{C}$ and $1000^{\circ} \mathrm{C}$, respectively, to desorb surface oxygen until gas evolution ceased, as indicated by the mass spectrometer (MS). Gasification was performed in the same TGA at $400^{\circ} \mathrm{C}$ in one atmosphere of pure $\mathrm{O}_{2}$. Post-gasification temperature programmed desorption (TPD) was performed on the samples in the same apparatus in flowing helium at $50 \mathrm{~K} / \mathrm{min}$ to $1100^{\circ} \mathrm{C}$. This entire procedure was repeated a number of times to obtain data at several burn-offs. For Sample 1, the entire procedure, including pyrolysis, was performed in the TGA. Sample 2 was pyrolyzed in a tube furnace and subsequently placed in the TGA.

2.3-2. Results and Discussion. The initial ${ }^{13} \mathrm{C} /{ }^{12} \mathrm{C}$ ratios for Samples 1 and 2 were determined by burning them completely in oxygen and integrating the corresponding $\mathrm{CO}$ and $\mathrm{CO}_{2}$ signals, as measured with the MS. (The labeled oxides are designated with an asterisk.) The resultant value of ${ }^{13} \mathrm{C} /{ }^{12} \mathrm{C}$ was about 0.12 for both samples. (The corresponding ratio for the natural abundance is 0.011 .) In the current discussion, $\mathrm{CO}$ denotes ${ }^{12} \mathrm{CO}$ and $\mathrm{CO} *$ denotes ${ }^{13} \mathrm{CO}$. In Figure 2.3-1 are presented the resultant reactivities of the two samples as a function of burn-off. As expected, the younger sample, Sample 1, shows consistently higher reactivity over the entire range of burn-off, especially at low burn-off. This is presumably due to the larger amount of amorphous material in the carbon structure. 


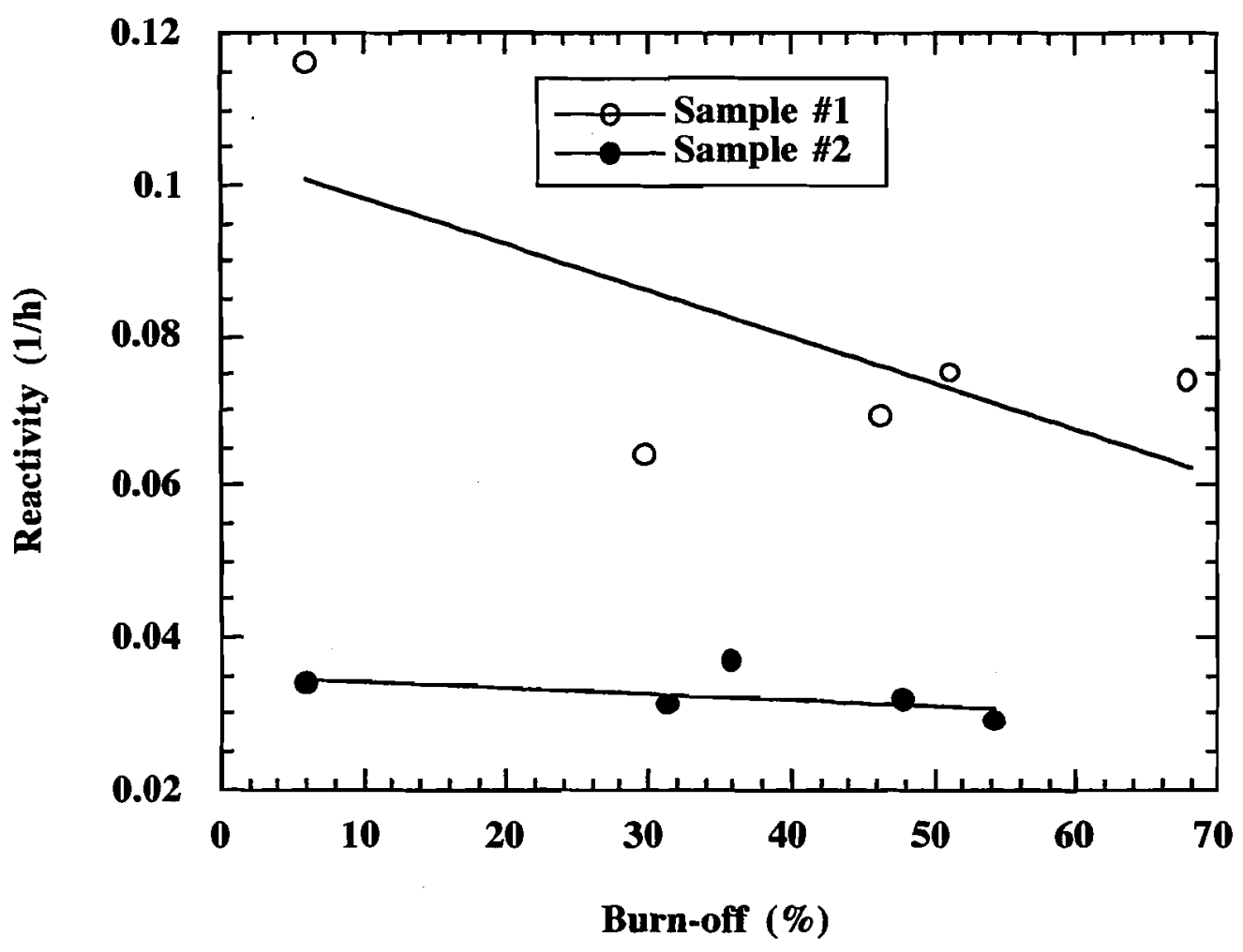

Figure 2.3-1. Oxygen reactivities at $400^{\circ} \mathrm{C}$ for the two resin char samples.

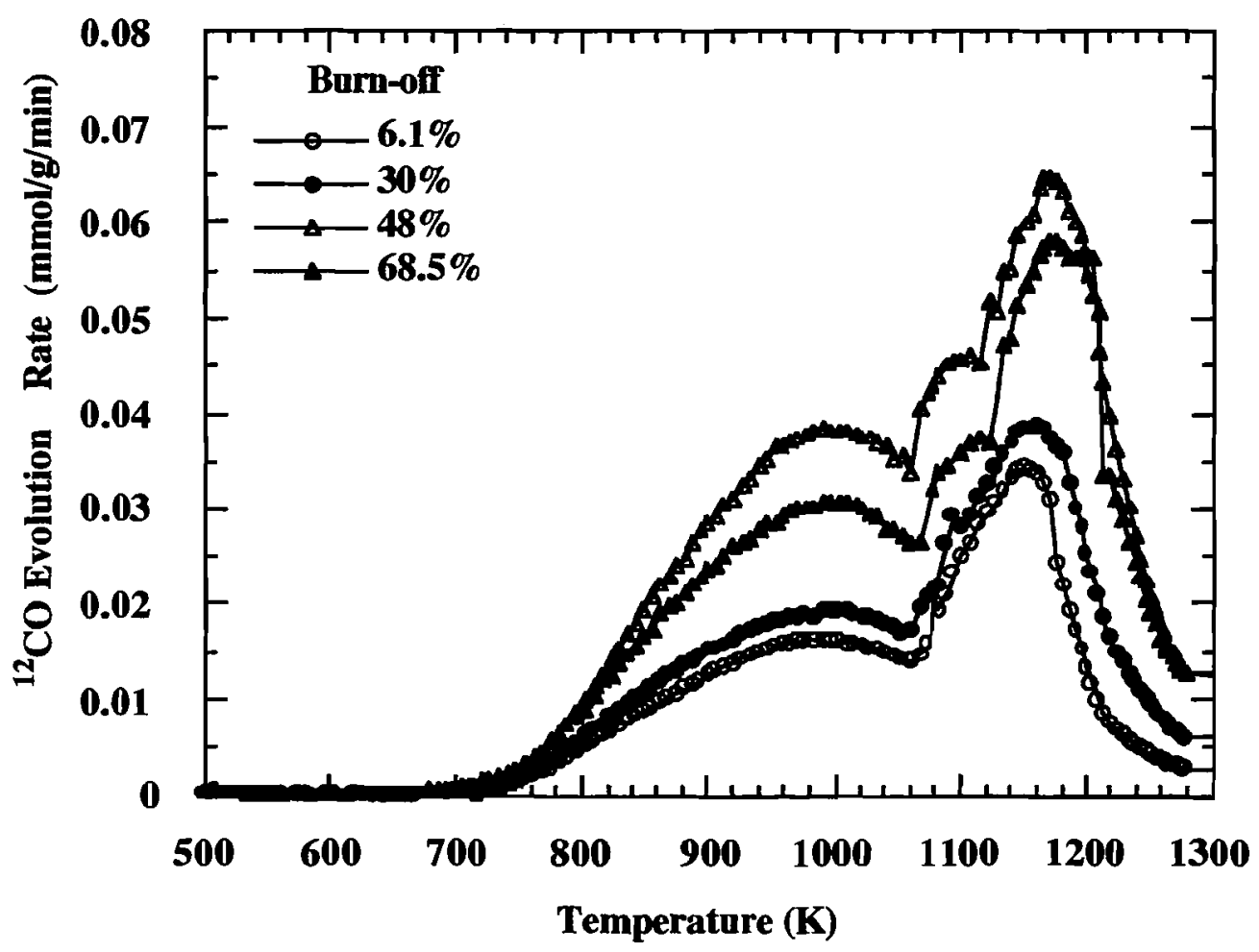

Figure 2.3-2. CO evolution rate from Sample 1 as a function of burn-off. 
In Figure 2.3-2 are presented the CO TPD spectra from Sample 1 as a function of burn-off. These results are somewhat different than others found in the literature, ${ }^{11}$ insofar as multiple desorption peaks were observed instead of the single broad peak obtained in the latter work. The TPD spectra in Figure 2.3-2 were deconvoluted to estimate the total integrated amount of surface complexes involved in each peak, as well as the peak temperatures. These results are presented in Table 2.3-1 as a function of burn-off for Sample 1. As shown, at low burn-offs, the spectra exhibit two peaks, which subsequently become three at higher burn-offs.

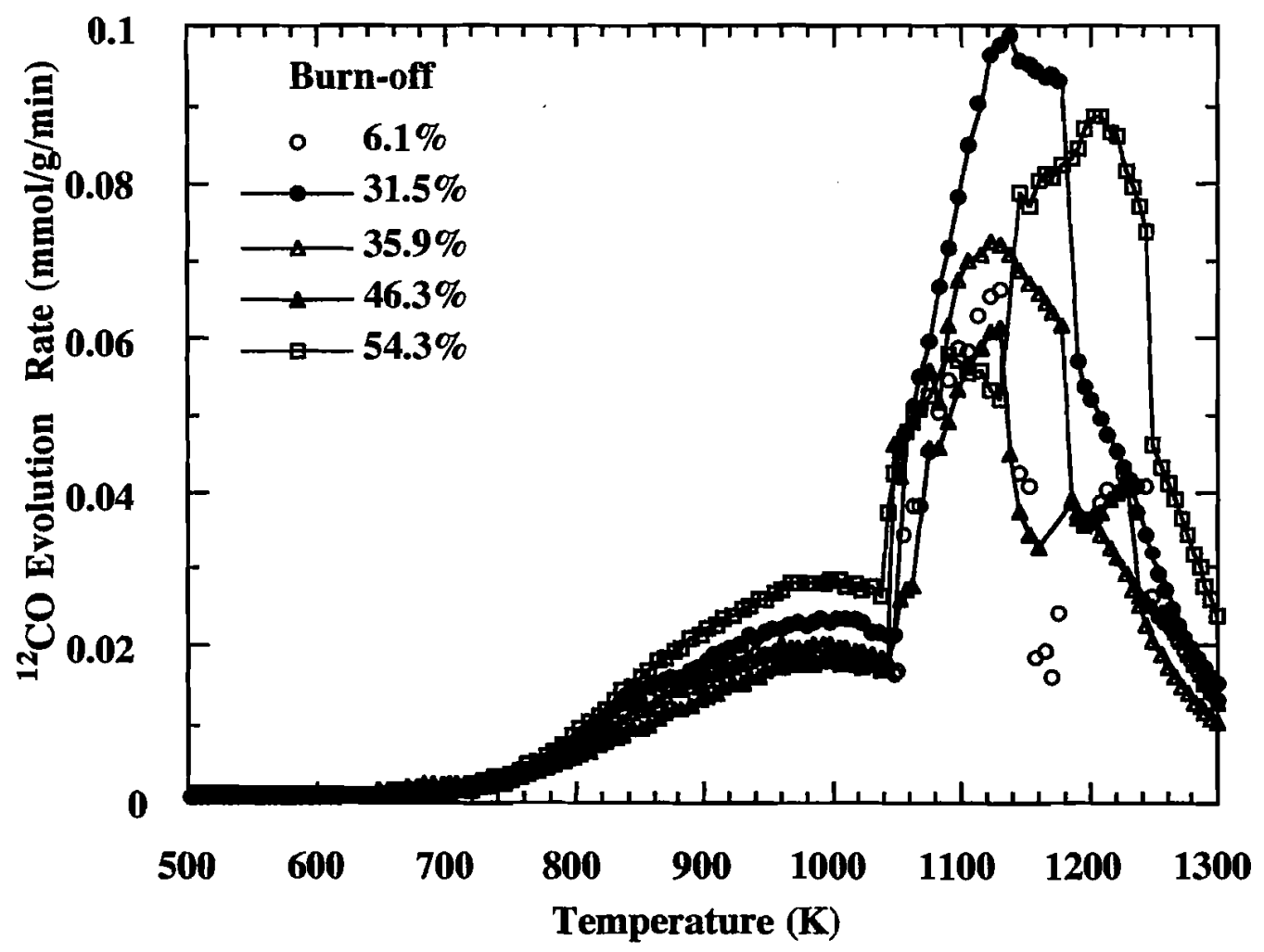

Figure 2.3-3. CO evolution rate from Sample 2 as a function of burn-off.

Figure 2.3-3 presents CO TPD spectra from Sample 2 as a function of burn-off. As shown, for Sample 2, three peaks were observed at all burn-offs. Companion experiments with an unlabeled resin char, prepared in the same fashion as Sample 2, revealed just two peaks at all burn-offs, similar to the results for Sample 1 at low burn-offs, and also to the results of Zhang with the same resin char. ${ }^{13}$ It appears that the two peaks observed for the unlabeled resin char and Sample 1 at early stages of burn-off are the same, and that the third peak observed for Sample 2 and during the later stages of gasification of Sample 1 occurs between the two original peaks. This third peak could be caused by a change in structure of the char due to heat treatment, since by the time the third peak appears for Sample 1, the char sample had been heated to $1400 \mathrm{~K}$ twice during TPD. This is also consistent with the fact that the third peak is present from the outset for Sample 2, which had been prepared at the higher temperature of $1300 \mathrm{~K}$. 
In Figures 2.3-4 and 2.3-5 are presented the corresponding evolution rate data of $\mathrm{CO}^{*}$ from Samples 1 and 2, respectively. As would be expected, a similar number of peaks is observed, but they are shifted to slightly higher peak temperatures. For Sample 1, both CO* and CO exhibit their first peak maxima at $1000 \mathrm{~K}$ and $993 \mathrm{~K}$, respectively. This is in agreement with the values obtained by Zhang ${ }^{13}$, who also observed two peaks at about the same temperatures for unlabeled resin char.

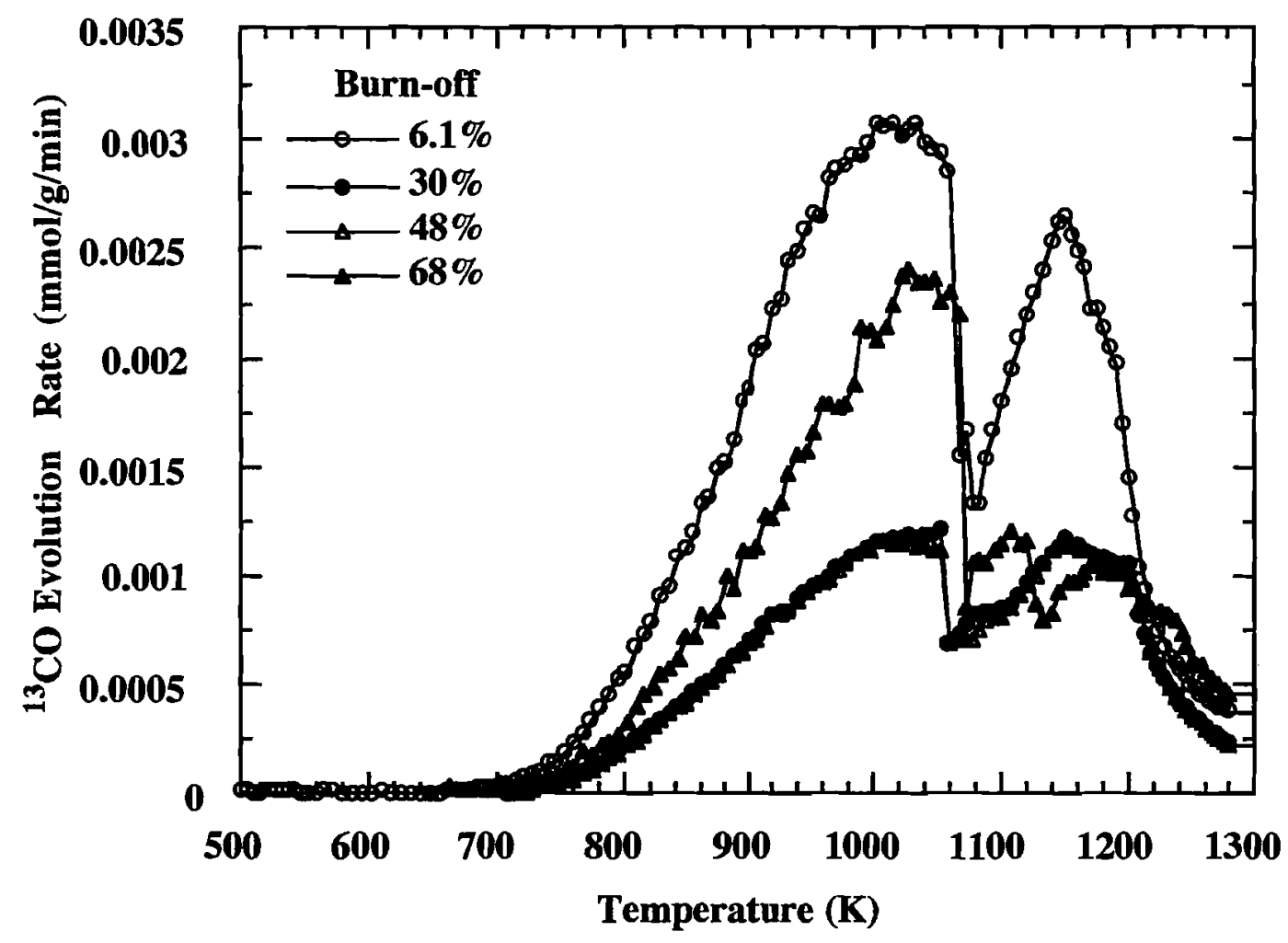

Figure 2.3-4. CO* evolution rate from Sample 1 as a function of burn-off.

Even more importantly, however, while the amount of evolved $\mathrm{CO}$ increases monotonically with burn-off until about $40 \%$ burn-off, and decreases thereafter (as was also observed by Zhang ${ }^{13}$ for unlabeled resin char), the trend is quite different for $\mathrm{CO}^{*}$. As presented in Table 2-2, the largest amount of total evolved $\mathrm{CO}^{*}$ occurs following the first burn-off. From this table, the corresponding $\mathrm{CO} * / \mathrm{CO}$ ratio is 0.15 for $6.1 \%$ burn-off, and it gradually decreases monotonically to 0.04 for $68.5 \%$ burn-off. This is consistent with the hypothesis that the ${ }^{13} \mathrm{CO}$ methylene bridge carbons in the original phenol-formaldehyde resin, which arise from the labeled formaldehyde, are preferentially enriched in the amorphous carbon material which burns off first before the more crystalline regions. The latter regions, on the other hand, arise primarily from the unlabeled aromatic phenol rings. 


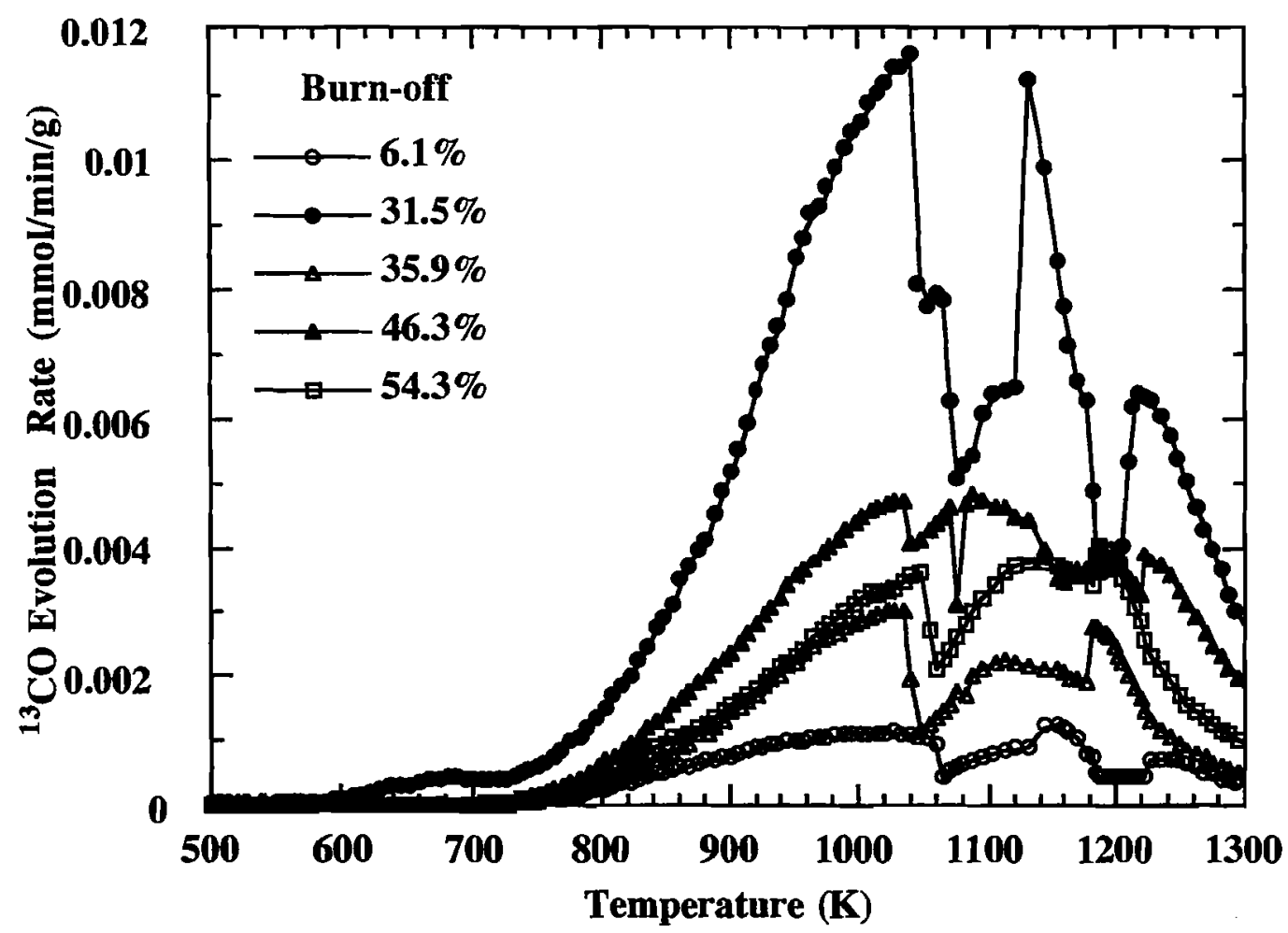

Figure 2.3-5. CO* evolution rate from Sample 2 as a function of burn-off.

The current char samples were observed to undergo large mass losses following TPD, which tended to decrease somewhat with progressive burn-off. In order to explore this behavior further, surface areas were determined by single point nitrogen BET at a relative pressure of 0.3 . The freshly pyrolyzed resin char exhibited a surface area of $372 \mathrm{~m}^{2} / \mathrm{g}$. Following $8 \%$ burn-off, the surface area increased to $444 \mathrm{~m}^{2} / \mathrm{g}$. However, following TPD of this same sample, the surface area increased markedly to $1233 \mathrm{~m}^{2} / \mathrm{g}$. The corresponding mass loss following TPD was $24 \%$. Both the surface area and mass loss are unusually large in comparison to those that have been more typically found for phenol-formaldehyde resin chars of this type in our laboratory. ${ }^{1,13}$ These results are consistent with a very high degree of microporosity which is blocked by oxygen surface complexes during gasification such that nitrogen at $77 \mathrm{~K}$ cannot penetrate. This microporosity then becomes accessible to nitrogen when these complexes are subsequently evolved during TPD. It is speculated that the larger than typical microporosity of these samples arose due to significantly greater cross linking of the resin structure during curing.

2.3-3. Conclusions. The process of gasification of a carbonaceous material has been investigated using a ${ }^{13} \mathrm{C}$-labeled phenol-formaldehyde resin as a model compound. The evolution patterns of CO upon post-reaction TPD are consistent with the early burn-off of amorphous carbon regions where ${ }^{13} \mathrm{C}$ originating from methylene bridges in the resin had concentrated, followed by attack of more crystalline aromatic regions. The unusually large amount of mass loss upon post-reaction TPD from these chars has been attributed to a well developed microporosity arising from a highly cross linked resin. 
Table 2.3-1. Peak temperatures (TP) and total integrated amounts of $\mathrm{CO}$ and $\mathrm{CO}^{*}$ desorbed (AP) in each peak for Sample 1.

\begin{tabular}{|c|c|c|c|c|c|c|c|c|c|c|c|c|}
\hline & $\mathrm{CO}$ & $\mathrm{CO}^{*}$ & $\mathrm{CO}$ & $\mathrm{CO}^{*}$ & $\mathrm{CO}$ & $\mathrm{CO}^{*}$ & $\mathrm{CO}$ & $\mathrm{CO}^{*}$ & $\mathrm{CO}$ & $\mathrm{CO}^{*}$ & $\mathrm{CO}$ & $\mathrm{CO}^{*}$ \\
\hline $\begin{array}{c}\text { Burn-off } \\
(\%)\end{array}$ & $\begin{array}{c}\mathrm{TP}(\text { Peak \#1) } \\
(\mathrm{K})\end{array}$ & $\begin{array}{c}\mathrm{AP}(\text { Peak } \\
\# 1) \\
(\mathrm{mmol} / \mathrm{g})\end{array}$ & \multicolumn{2}{|c|}{$\begin{array}{c}\mathrm{TP} \text { (Peak \#2) } \\
(\mathrm{K})\end{array}$} & $\begin{array}{c}\text { AP (Peak } \\
\# 2) \\
(\mathrm{mmol} / \mathrm{g})\end{array}$ & \multicolumn{2}{|c|}{$\begin{array}{c}\text { TP (Peak \#3) } \\
(\mathrm{K})\end{array}$} & $\begin{array}{c}\text { AP (Peak } \\
\# 3) \\
(\mathrm{mmol} / \mathrm{g})\end{array}$ \\
\hline 6.1 & 993 & 1000 & 93.5 & 16.8 & 1152 & 1170 & 53.0 & 5.0 & - & - & - & - \\
\hline 30 & 993 & 1000 & 112.4 & 6.9 & 1155 & 1180 & 63.8 & 2.9 & - & - & - & - \\
\hline 48 & 993 & 1000 & 235.4 & 11.6 & 1105 & 1100 & 25.9 & 5.5 & 1180 & 1205 & 106.3 & 5.8 \\
\hline
\end{tabular}

\subsection{The "Loss of Memory" Effect on Char Reactivity.}

One of the fundamental premises of the current project is that char reactivity is significantly affected by structural changes in the carbon structure as a consequence of heat treatment and annealing. It is also suspected, however, that reaction itself can also play a role in determining the structure of the carbon. For example, it has been well established that kinetically-limited gasification does not always occur at constant particle diameter, but rather may be accompanied by gasification-induced atomic rearrangements, which in some chars result in loss of pore volume and carbon particle shrinkage. ${ }^{14,15}$ Breaking bonds, removing cross links, and removing carbon atoms, increases the driving force for, and facilitates char densification via rearrangements analogous to those occurring spontaneously at somewhat higher temperatures via thermal annealing. If the net effect of this mechanism is significant enough, it can cause the reactivity of carbon materials which initially may be considerably different to ultimately be quite similarly; that is, they can lose the memory of their precursor state.

2.4-1. Experimental. Coal samples were obtained from the Penn State Coal Sample Bank, and the phenol-formaldehyde resin was synthesized in our laboratory. ${ }^{1}$ Chars were prepared in a tube furnace in flowing ultra-high purity helium at $900^{\circ} \mathrm{C}$ and $1300^{\circ} \mathrm{C}$ for 1.5 and 4 hours, respectively, for each material. The final particles used were 30-100 Tyler mesh. Gasification was performed in a TGA in one atmosphere of pure $\mathrm{O}_{2}$ at the indicated temperatures. The samples were burned out completely, and the amount of residual ash (for the coal chars) was used to determine fractional conversion on a carbon basis. Samples were characterized for pore size distributions and surface areas from $77 \mathrm{~K}$ nitrogen isotherms.

Selected samples were subjected to thermal desorption following reaction in order to investigate the behavior of the population of intermediate oxygen surface complexes with respect to elucidating the mechanisms responsible for the observed reactivity behavior. These experiments were performed in the same apparatus at a heating rate of $50 \mathrm{~K} / \mathrm{min}$ following a reactivity determination. The evolution rates of $\mathrm{CO}$ and $\mathrm{CO}_{2}$ upon thermal desorption were recorded with a quadrupole mass spectrometer. 
2.4-2. Results. In Figure 2.4-1 are presented the reactivity and reaction rate behavior of the two Pocahontas \#3 coal char (POC) samples. As shown, both rate measures approach one another asymptotically at high burn-offs. Pocahontas \#3 (DECS-19), is a low volatile bituminous coal with about $4.55 \%$ ash (AR basis). Consequently, it is possible that the approach of the rate curves to one another may be due to improvement in the carbon-mineral matter interfacial contact due to progressive concentration of mineral matter with burn-off, and eventual domination of catalytic effects in controlling sample reactivity. As shown in Figure 2.4-2, however, the phenolic resin char (PRC) samples exhibit the same qualitative behavior as the POC samples. In this latter case, however, the behavior cannot be ascribed to catalysis, since the mineral matter content of this material is insignificant.

Figure 2.4-3 also exhibits the same qualitative trend for Beulah coal char (BEU) samples. Beulah (DECS-11) is a lignite coal with about 6.37\% ash (AR basis). Although Figures 2.42 and 2.4-3 are qualitatively similar, the rate curves for the BEU char do not merge as rapidly as for the PRC at high burn-offs. In Figure 2.4-4 are presented similar results for Pittsburgh coal char. Pittsburgh coal (DECS-23) is a high volatile bituminous coal with about $9.25 \%$ mineral matter (AR basis). Just as for the BEU char, the rate curves do not merge as rapidly as for the PRC and POC chars. Pittsburgh coal undergoes a fluid phase upon slow pyrolysis such that most of the porosity of the original coal is lost, which explains the very similar reactivities at low-burn-offs. As shown, the reactivity curves subsequently diverge quite considerably, however, due to different rates of porosity evolution, presumably controlled by the different morphologies of the two samples.

Post-reaction, CO thermal desorption spectra are presented in Figure 2.4-5 for selected PRC samples, and Figure 2.4-6 for selected POC samples. These reveal some interesting trends. For both types of chars, the oxygen surface coverage is considerably greater for the $900^{\circ} \mathrm{C}$ ("younger") samples than for the $1300^{\circ} \mathrm{C}$ ("older') samples. This is consistent with other indications that the "younger" chars have more disordered carbon associated with them, and, consequently, a greater number of sites for oxygen chemisorption. However, it is well-known ${ }^{16}$ that the overwhelming majority of these oxygen surface complexes are not active in the gasification reaction; rather it is only a small fraction of the lowest temperature complexes with the lowest desorption activation energies that effectively contribute to reactivity. In this regard, it is noted that the desorption spectra for the high burn-off cases for both chars appear to converge more closely at low temperatures than the low burn-off samples. This is evident in the "blow-up" of the low temperature region in Figure 2.45 (b) and also in Figure 2.47. This is consistent with the reactivities being more similar at high burn-offs than at low burn-offs. 


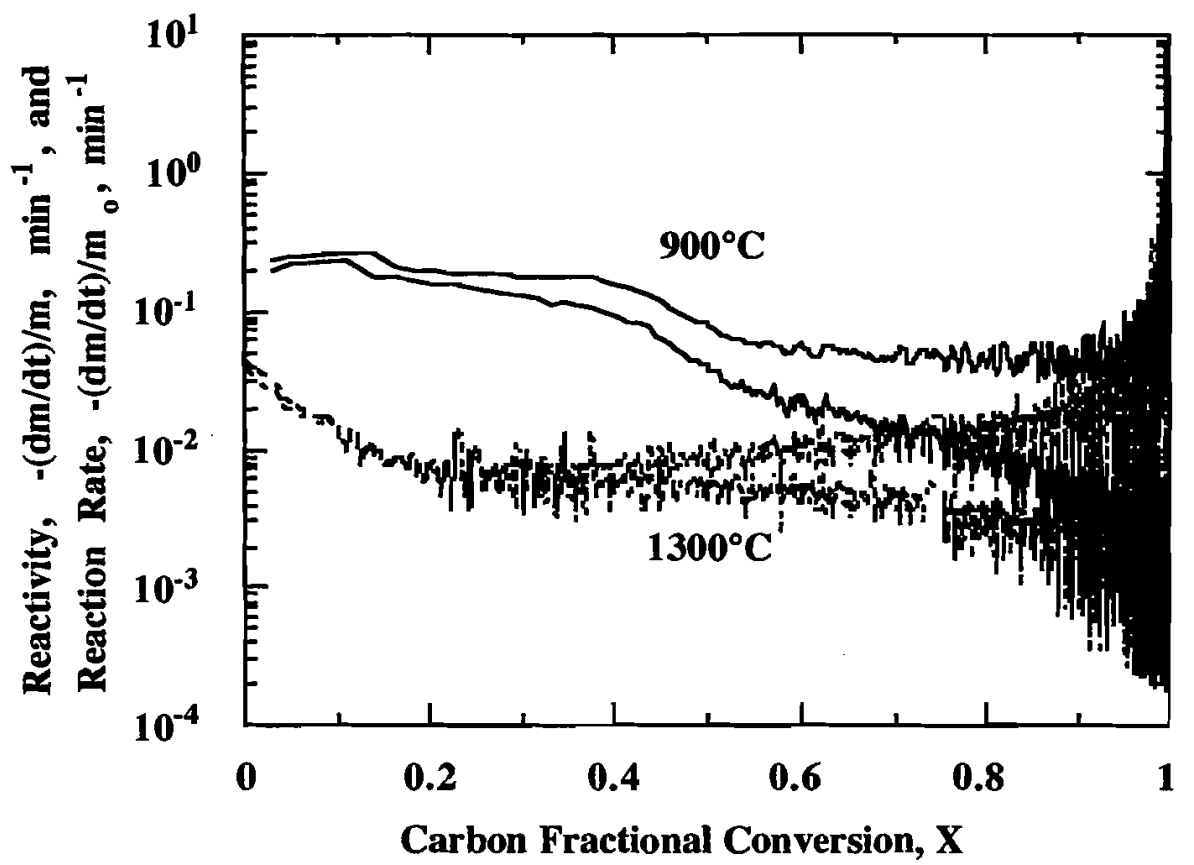

Figure 2.4-1. Reactivities of $900^{\circ} \mathrm{C}$ and $1300^{\circ} \mathrm{C}$ Pocahontas coal char samples in $\mathrm{O}_{2}$ at $650^{\circ} \mathrm{C}$.

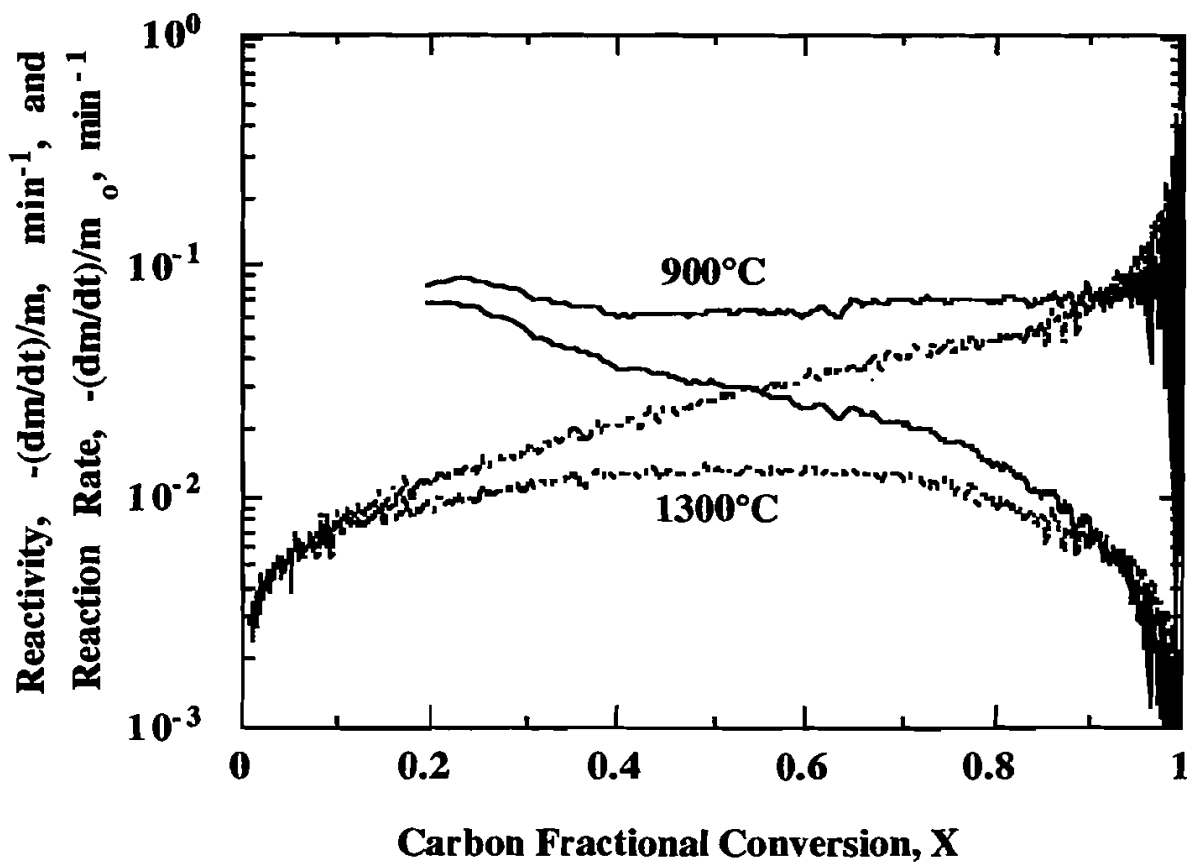

Figure 2.42. Reactivities of $900^{\circ} \mathrm{C}$ and $1300^{\circ} \mathrm{C}$ phenolic resin char samples in $\mathrm{O}_{2}$ at $500^{\circ} \mathrm{C}$. 
Potentially, there are at least three major mechanisms that may be involved in determining the observed behavior; i.e., the effects of (1) porosity and surface area evolution; (2) degree of carbon ordering; and (3) mineral matter impurities. As already indicated, in the case of the PRC and POC samples, catalytic effects do not provide a suitable explanation. Normalization of the reactivities for surface area also does not account for the observed differences at low to moderate burn-offs. For example, the surface areas for the two POC samples are practically the same at low burn-offs and yet the reactivity of the $900^{\circ} \mathrm{C}$ sample is about five times that of the $1300^{\circ} \mathrm{C}$ sample. The surface areas of the two PRC samples are almost the same throughout the entire burn-off history, yet the reactivity of the $900^{\circ} \mathrm{C}$ sample is as much as an order of magnitude greater than that of the $1300^{\circ} \mathrm{C}$ at low burn-offs. And finally, the surface areas of the $1300^{\circ} \mathrm{C} \mathrm{BEU}$ char are about twice as large as those of the $900^{\circ} \mathrm{C}$ sample over most of the burn-off range, and yet the reactivity of the $900^{\circ} \mathrm{C}$ sample is considerably greater.

In view of the preceding, it is proposed that the observed behavior is largely attributable to the evolution of the structural morphology of the samples. HRTEM fringe image analysis ${ }^{2}$ indicates that although in each case the samples are quite different at low burn-offs, they become more similar at high burn-offs. Although this is a complex process, it appears that a major factor is selective burn-off of less ordered material at the expense of more highly ordered structures ${ }^{17}$, such that eventually the morphology becomes similar enough that reactivity differences are minimized. This is supported by the thermal desorption data which indicate that the portion of the active sites responsible for reactivity appear to become more similar at high burn-offs for both the "younger": and "older" char samples. These effects may also be related to recent observations of Salatino and co-workers ${ }^{18}$ at higher temperatures where continued growth of ordered regions may also contribute to this effect. 

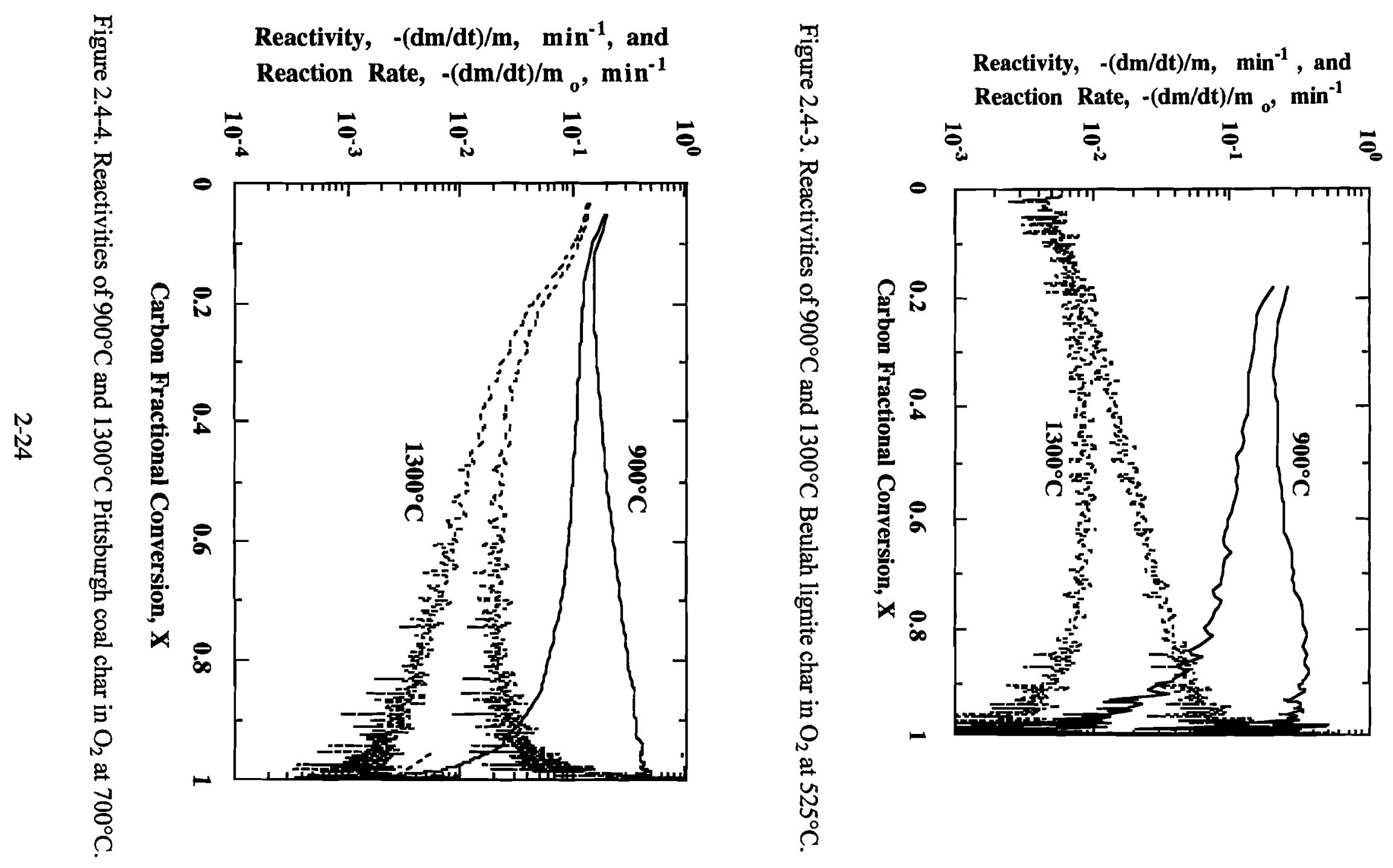


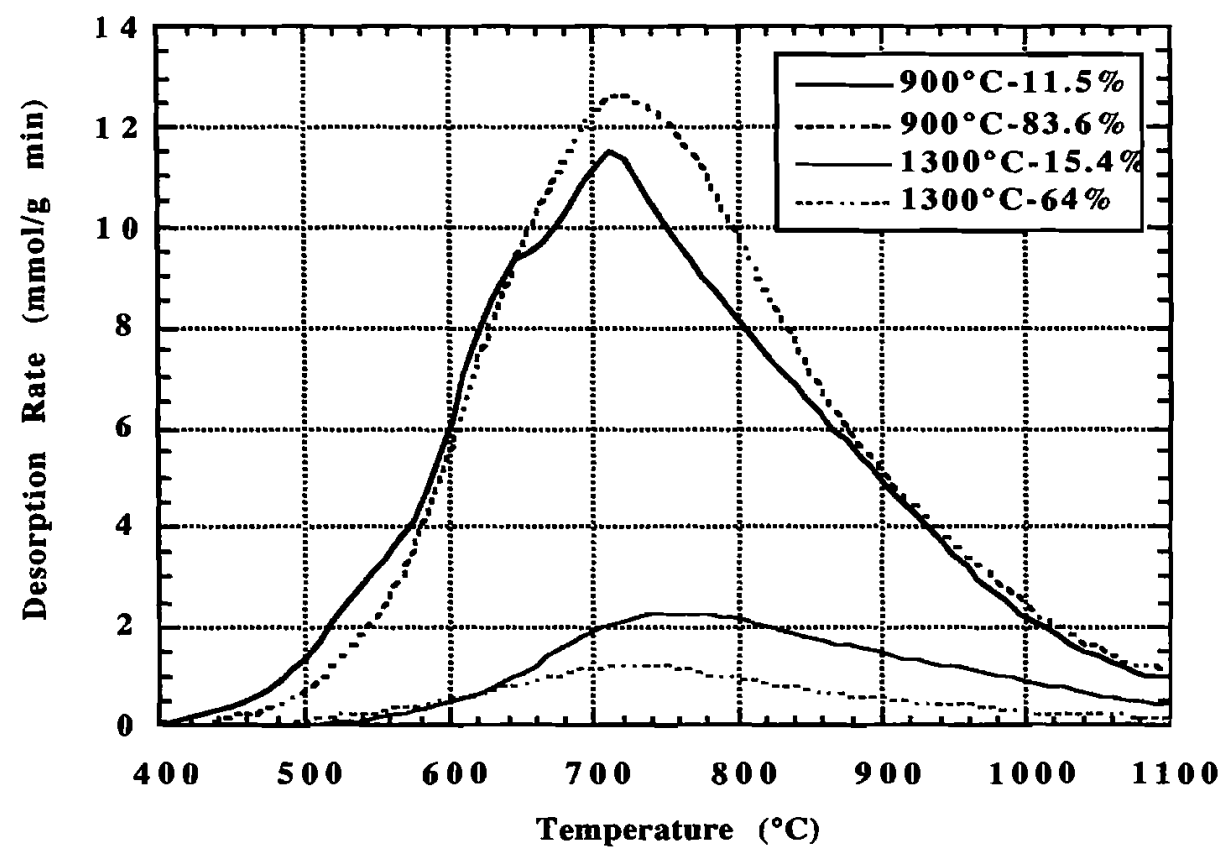

Figure 2.4-5 (a). CO desorption spectra $(50 \mathrm{~K} / \mathrm{min})$ of selected phenolic resin char samples. The corresponding pyrolysis temperatures $\left(900^{\circ} \mathrm{C} / 1.5 \mathrm{~h} ; 1300^{\circ} \mathrm{C} / 4 \mathrm{~h}\right)$ and burn-off at $500^{\circ} \mathrm{C}$ in oxygen are indicated in the legend.

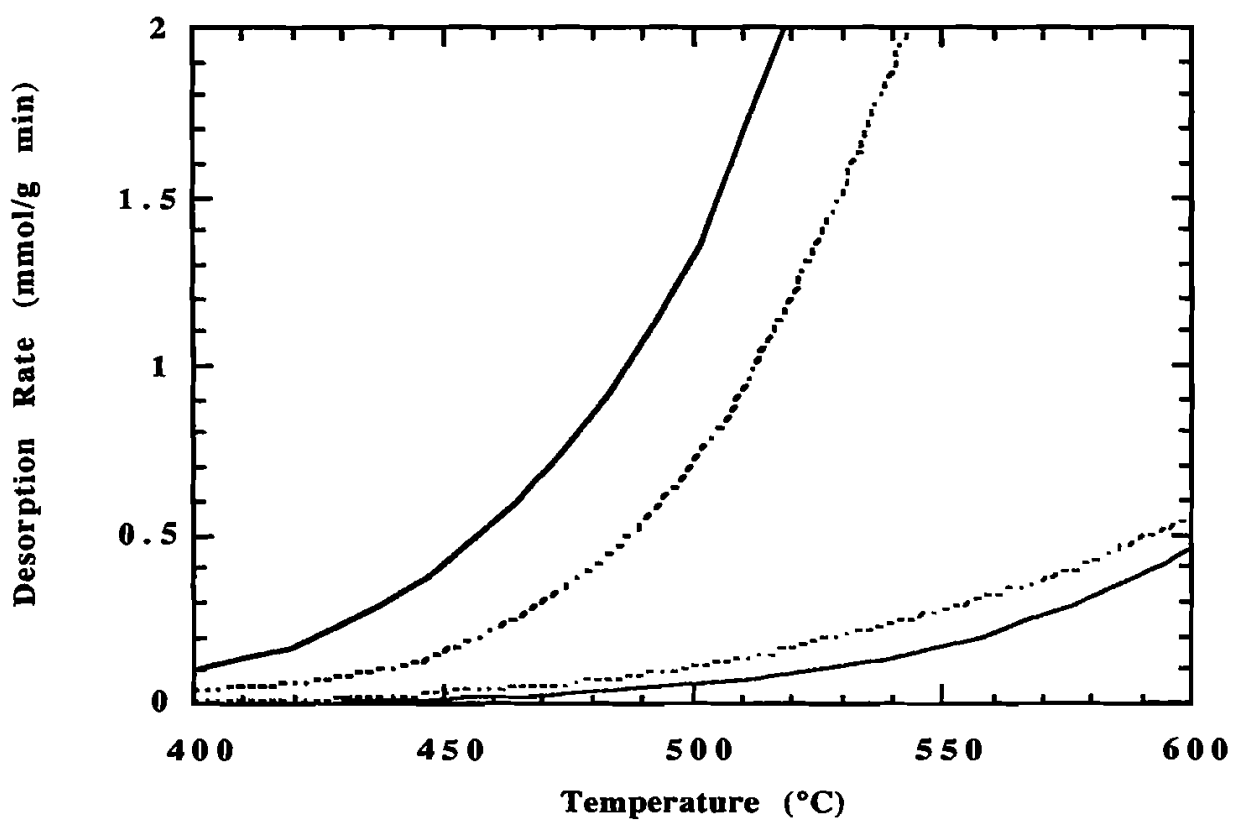

Figure 2.4-5 (b). Blow-up of low temperature region in Figure 2.4-5 (a). 


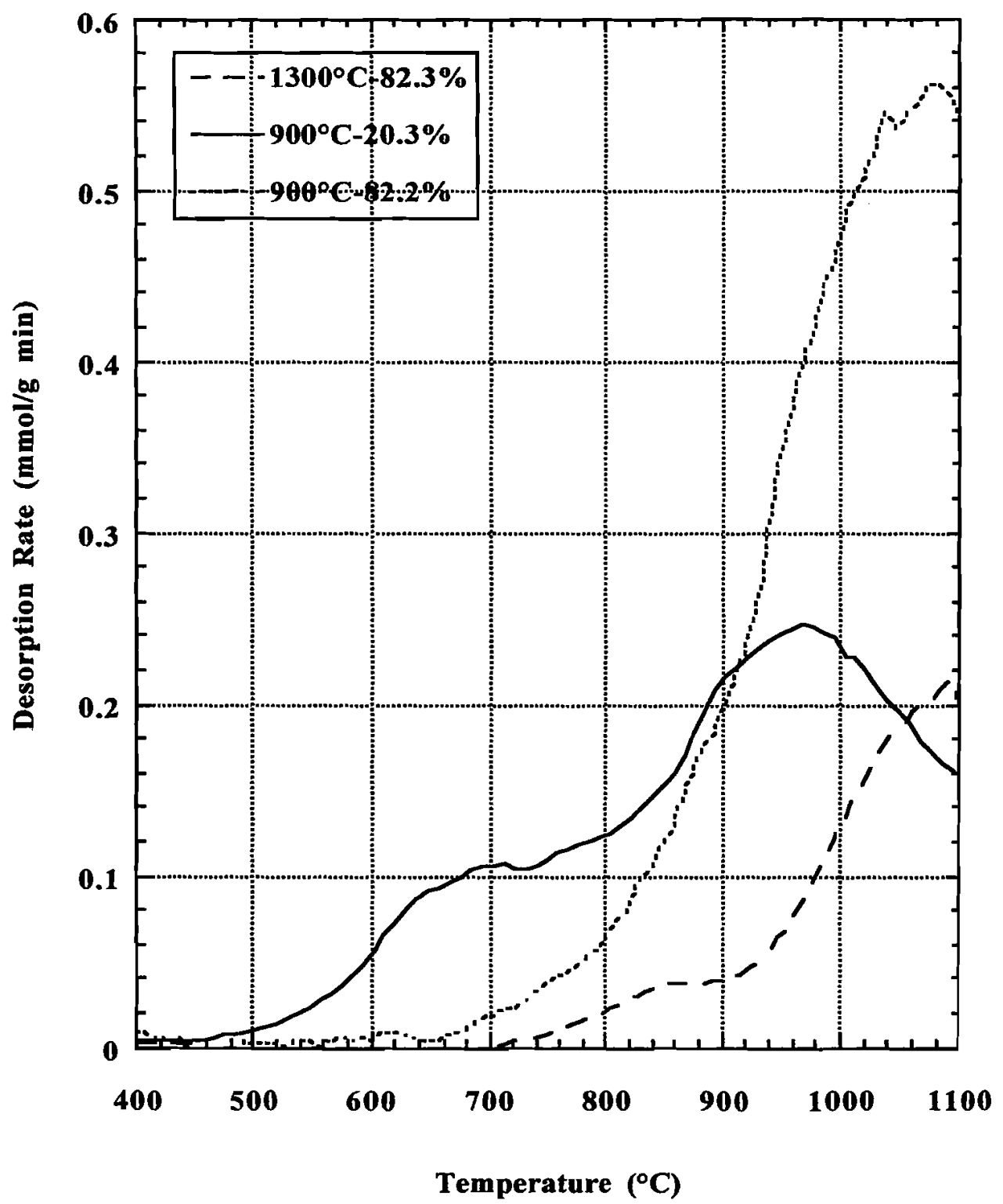

Figure 2.4-6. CO desorption spectra (50K/min) of selected Pocahontas coal char samples. The corresponding pyrolysis temperatures $\left(900^{\circ} \mathrm{C} / 1.5 \mathrm{~h} ; 1300^{\circ} \mathrm{C} / 4 \mathrm{~h}\right)$ and burn-off at $500^{\circ} \mathrm{C}$ in oxygen are indicated in the legend. 


\subsection{Char Porosity Characterization Via Small Angle Neutron Scattering.}

2.5.1. Introduction. Porosity and porosity development are intimately involved in determining the resultant reactivity of coal chars. ${ }^{19}$ In particular, accessibility of gases to the porosity ${ }^{20,21}$ during combustion and/or gasification ${ }^{22}$ are critical. In this context, there is an important distinction between porosity that is connected to the exterior of the particles, and porosity that is effectively inaccessible to fluid transport from the exterior. During the gasification process, accessible porosity may be widened, previously inaccessible porosity may be opened, and new porosity may be developed. ${ }^{23}$ Thus, the presence of "closed" or inaccessible porosity can have a significant effect on how porosity develops. Despite its importance, however, this process is still not well understood, primarily because there are only a limited number of available techniques that can be used to accurately characterize "closed" porosity.

A number of techniques have been applied to the investigation of porosity in carbons in general. These include gas adsorption ${ }^{24,25,26}$, mercury intrusion porosimetry, $\mathrm{TEM}^{27}$, and small angle scattering techniques ${ }^{28,29}$. By their very nature, gas adsorption and mercury intrusion porosimetry do not provide information about "closed" porosity, and TEM only gives limited information concerning pore connectivity ${ }^{30}$. Small angle $\mathrm{X}$-ray scattering provides scattering data from both "open" and "closed" porosity, which cannot be decoupled.

We have applied contrast matching small angle neutron scattering (CM-SANS) to investigations of "closed" porosity in a variety of materials ${ }^{31,32,33}$. The technique consists of performing SANS on "dry" samples where the total scattering is from all the porosity, including both accessible ("open") and inaccessible ("closed") pores. The pores accessible from the exterior of the sample are then filled with a liquid that has the same neutron scattering cross section as the solid matrix, such that when SANS is performed on the "wet" sample, scattering from the "open" porosity is eliminated. Consequently, scattering from the contrast-matched sample yields information about the remaining, inaccessible porosity in these materials. Moreover, the difference in scattering between the corresponding dry and contrast-matched samples provides information about the "open" porosity that may be present.

Here we focus on contrast matching studies of two very different chars produced by the pyrolysis of Wyodak subbituminous coal and a phenolic resin. Unactivated Wyodak coal char is known to exhibit some microporosity without activation, but its porosity development history, and the role of closed porosity is not well known. Phenolic resin forms a low density and highly microporous char. The surface area of the unactivated char is generally very low $\left(<2 \mathrm{~m}^{2} / \mathrm{g}\right)$. It has been noted, however, that low degrees of activation $c a$. 1-2\% significantly increase the BET surface area of these materials. In order to account for this behavior, it has been hypothesized that phenolic resin char (PRC) has a large amount of closed porosity in its unactivated form that is opened during subsequent activation.

2.5-2. Background. Analysis of small angle scattering yields a number of useful parameters that can be used for porosity characterization; one of these is the Porod Invariant $(Q)$, which is defined $\mathrm{as}^{34}$ : 


$$
Q=\int_{0}^{\infty} q^{2} I(q) d q,
$$

where $I(q)$ is the scattering intensity at a value of the scattering wave vector, $q$,

$$
q=4 \pi \sin (q) / \lambda,
$$

$\lambda$ is the neutron wavelength and $\theta$ is the scattering half-angle. These are related to material parameters via:

$$
2 \pi^{2} b_{v}^{2} \phi_{s}\left(1-\phi_{s}\right)=(1 / V) \int_{o}^{\infty} q^{2} I(q) d q,
$$

where $\phi_{s}$ is the volume fraction of the solid material, $b_{v}$ is the contrast factor between the solid material and the void (pores) per unit volume, and $V$ is the sample volume under consideration. The relationship between $\phi_{\mathrm{s}}$ and the interfacial scattering area depends upon the assumption of a pore geometry and a size distribution. In general, this relationship is complex, especially if the scatterers are polydisperse.

A number of models have been used to interpret scattering data from porous materials. Hall et al. ${ }^{35}$ used a combined fractal and fully penetrable polydisperse spheres (FPPS) model to describe the porosity of microporous polymers. The fractal portion of the model was used to describe scattering in the low $q$ range attributable to meso- and macroporosity, while a unimodal distribution of FPPS was used to describe the contribution from microporosity at larger $q$ values. Foster and Jensen ${ }^{36}$ used a unimodal FPPS model to describe the porosity of a commercial alumina catalyst support.

For fractal models, Texeira ${ }^{37}$ and Schmidt et al ${ }^{38}$ have shown that the scattering intensity is given by:

$$
I(q)=I_{0} \Gamma(5-D) \sin [\pi(D-1) / 2] q^{-(6-D)}
$$

where $I_{o}$ is a constant, $\Gamma$ is the gamma function, and $D$ is the surface fractal dimension. For perfectly smooth scattering surfaces, the scattering intensity will follow a Porod distribution (I(q) $\alpha$ $\left.q^{-4}\right),{ }^{39,40}$ and the resultant fractal dimension will be $D=2$. Consequently, the more fractal the scattering surface, the less steep the slope of the scattering curve.

The total scattering intensity is given by:

$$
\mathrm{I}(\mathrm{q})=\mathrm{V}\left(\mathrm{b}_{\mathrm{v}}{ }^{2}\right) \phi_{\mathrm{s}}\left(1-\phi_{\mathrm{s}}\right) \int_{\mathrm{o}}^{\infty} \gamma(\mathrm{r}, \mathrm{p})(\sin (\mathrm{qr}) / \mathrm{q} r) 4 \pi \mathrm{r}^{2} \mathrm{dr},
$$

where the correlation function, $\gamma(r, p)$ for a model with parameter values $p$ represents the probability that a point at distance $r$ in an arbitrary direction from a point in a given phase will also be in that phase. The correlation function is closely related to the two-point conditional probability function $\mathrm{S}_{2}(\mathrm{r} ; \mathbf{p})$ :

$$
\gamma(r, p)=\left[S_{2}(r ; p)-\phi_{s}{ }^{2}\right] /\left[\phi_{s}\left(1-\phi_{s}\right)\right] .
$$

$S_{2}\left(r ; f(R), \phi_{s}\right)$ has been derived for a general, unimodal distribution [16]. The evaluation of $S_{2}(r$; $\left.f(R), \phi_{s}\right)$ involves integrations over the frequency distribution, $f(R)$. Hence, the application of Eq. [5] to calculate the scattering intensity requires a double integration over r. One useful frequency distribution for the sphere sizes, examined by Foster and Jensen [16], is the Schulz distribution:

$$
f(R)=(1 / \Gamma(b))\left(b / R_{o}\right)^{b} R^{b-1} \exp \left(-b R / R_{o}\right)
$$


where $R_{o}$ is the mean sphere radius and $b$ is a parameter that increases with the sharpness of the distribution; e.g., an inverse variance. The requisite numerical integration for the evaluation of $S_{2}(r$; $\left.f(R), \phi_{s}\right)$ can be simplified by making use of the fact that the integrals in the two-point conditional probability function for the Schulz distribution can be represented analytically by incomplete gamma functions which are related to one another by simple recursion relationships, such that only one incomplete gamma function, $P(b, x)$, (where $x=b r / 2 R_{o}$ ) and $\Gamma(b)$ need be evaluated for each value of $x$. This essentially eliminates the numerical quadratures for the inner integrations required to calculate $S_{2}(r)$.

In addition to the fractal-unimodal FPPS model, a bimodal FPPS model can also be used to interpret the scattering intensity data in terms of larger (e.g., macro/meso-) and smaller (e.g., micro-) porosity. The latter model assumes that the distribution of FPPS is represented by a linear combination of two modes ${ }^{41}$; i.e.,

$$
f(R)=\varepsilon_{1} f_{1}(R)+\varepsilon_{2} f_{2}(R)
$$

where the $\varepsilon_{\mathrm{i}}$ are the relative weights of the two modes, and $\Sigma \varepsilon_{1}=1$. In the current work, we also fit some of the data to this latter model using a correlation function constructed from a bimodal Schulz distribution.

In general, the model prediction of $\mathrm{I}(\mathrm{q})$ must be determined numerically and the reconciliation of the experimental and calculated scattering intensities then requires nonlinear regression. Although various objective functions can be formulated, in order to more equally weight evenly spaced data (in q), Foster and Jensen ${ }^{42}$ suggested an objective function based on the logarithms of the intensities:

$$
\begin{gathered}
\mathrm{F}=\sum_{\mathrm{i}=1}\left[\ln \left(I^{*}(\mathrm{q})\right)-\ln (\mathrm{I}(\mathrm{q}))\right]^{2}, \\
\text {, }
\end{gathered}
$$

where the $I^{*}(q)$ and the $I(q)$ are the experimental and calculated scattering intensities, respectively, and $\mathrm{N}$ is the total number of experimental values. A nonlinear least squares fitting algorithm can then be used to evaluate the model parameter set which minimizes the objective function, F. For the current purposes, a Levenberg-Marquardt, or Marquardt method, was selected . ${ }^{43}$

2.5-3. Experimental. The SANS data were collected at the Intense Pulsed Neutron Source (IPNS) of the Argonne National Laboratory using the small angle diffractometer (SAD) ${ }^{44} \mathrm{~A} \quad 64 \times 64$, position sensitive multidetector was used to detect scattered neutrons, and the wavelength of the neutrons was determined by time-of-flight. The sample holders were made of Suprasil with a pathlength of $0.2 \mathrm{~cm}$. Scattering data were corrected for scattering from the sample holder and other instrumental backgrounds. Normalization for the sample thickness and transmission was made, and the data were scaled to yield absolute calibrations. Transmission measurements indicated no multiple scattering effects with the $2 \mathrm{~mm}$ cells for none of the samples. The entire available $\mathrm{q}$ range $\left(0.005<q<0.35 \AA^{-1}\right)$ of the small angle diffractometer was used.

Wyodak subbituminous coal samples were obtained from the Argonne Premium Coal Sample Bank. ${ }^{45}$ Phenolic resin was produced by the method described in detail by Sandler and Karo. ${ }^{46}$ 
Char samples were prepared from these precursors by pyrolysis under a slight positive pressure of nitrogen. Samples were heated at a rate of $10 \mathrm{~K} / \mathrm{min}$ to $1173 \mathrm{~K}$ for the phenolic resin, and $1273 \mathrm{~K}$ for the coal, with a heat soak time of 1 hour. The resultant char samples were ground to between 60 and 100 Tyler mesh. Activation of the coal char was accomplished in air in a tube furnace at $673 \mathrm{~K}$, and in oxygen at $743 \mathrm{~K}$ for the phenolic resin char.

The surface areas of the chars were determined via single point BET analysis of nitrogen adsorption data at $77 \mathrm{~K}$ in a Quantachrome Quantasorb apparatus. The burn-offs and corresponding single point nitrogen BET surface areas are presented in Table 2.5-1 for the six Wyodak coal char samples, and Table 2.5-2 for the four phenolic resin chars samples.

It is noted that the surface areas are relatively low for a resin char produced at $900^{\circ} \mathrm{C}$. They are actually more typical of a PRC produced at much higher temperatures (e.g., at $1800-2400^{\circ} \mathrm{C}^{47}$ ). The curing process used for the precursor resin, however, produced a highly cross-linked material, which is consistent with the behavior of the BET surface area. Moreover, a highly crosslinked precursor is also consistent with a significant amount of disordered carbon in the resultant char (see below).

The neutron scattering density of carbon is $5.6 \times 10^{10} \mathrm{~cm}^{-2}$, which is very close to that of perdeuterated toluene. Consequently, it is used for contrast matching in the current experiments. Essentially, char samples were mixed in powdered form with perdeuterated toluene and allowed to come to equilibrium by immersion in an ultrasonic bath for four hours.

\section{5-4. Results and Discussion.}

2.5-4.1. Wyodak Coal Char. In Figure 2.5-1 are presented the scattering intensity curves from the six "dry" Wyodak char samples. As shown, the total scattering increases with activation up to 58\% burn-off, and then decreases at $68 \%$ burn-off over the entire $\mathrm{q}$ range, but especially in the micropore region for $q>0.1 \AA^{-1}$. These data are similar to SAXS data from Carbosieve-S gasified in $\mathrm{CO}_{2}$, which exhibited increased scattering intensity with progressive activation up to $30 \%$ burn-off, but then decreased with further gasification . $^{48}$

Two regimes of behavior are apparent in Figure 2.5-1: one at low burn-off $(0,7.4$, and 9.2\%), which all appear to be quite similar; and the other at high burn-off $(32.7,58$, and $68 \%)$. The three lowburn-off data sets were fit to the fractal/FPPS single mode Schulz distribution model presented above. The resultant slopes of the linear region at low $q$ for the three samples were all very close to 4, which is characteristic of Porod scattering ${ }^{49}$; i.e., a fractal dimension of $D=2.0$. The corresponding Schulz distribution parameters from the high q data changed from a mean of $24.5 \AA$ to $18.2 \AA$, both with a variance $\left(R_{o} / b\right)$ of $4 \AA$.

The contrast matched and "difference" curves, corresponding to the same samples in Figure 2.5-1, are presented in Figure 2.5-2. At low q this regime of behavior is typified by the "difference" curves all lying above their corresponding contrast matched curves. Moreover, the "difference" curves are all practically the same, and the contrast-matched curves are quite similar as well. This 
indicates that at low burn-offs the larger porosity is mostly open, and that it does not change very much with activation. In this region, the "difference" curves are also well described by Porod

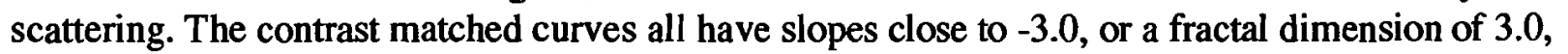
as from very convoluted surfaces.

At high q, however, the situation is reversed, with the contrast matched curve lying above the "difference" curve in each case. Moreover, the contrast matched curve first increases and then decreases, while the "difference" curve first decreases and then increases with burn-off, making the absolute difference between contrast matched and "difference" curves greatest for the $7.4 \%$ burnoff sample. This is interpreted in terms of three processes occurring simultaneously in the microporosity: creation, consumption via gasification, and conversion of initially closed to open microporosity via consumption of carbon blocking pores. This is consistent with the following qualitative picture of the activation process with respect to the micropores. Initially there is more closed than open microporosity. With activation, however, there is additional growth of closed porosity, and a net decrease in open porosity. This may be due to some residual pyrolysis that creates some amorphous carbon that blocks pores that were already open; and/or some open pores were consumed while residual pyrolysis created some additional closed porosity. The actual situation may be a combination of both. However, the fact that the contrast matched curve increases significantly at low $\mathrm{q}$ from the unactivated to the $7.4 \%$ burn-off sample, is consistent with the creation of large scatterers of amorphous carbon due to residual pyrolysis (see the discussion below for phenolic resin char). In any case, by $9.2 \%$ burn-off, the two curves are closer together, almost to where they were in the unactivated sample, suggesting that there is still some closed microporosity but that most of it is open.

For the unactivated sample, fits of the scattering curves in Figure 2.5-2 to the fractal/single mode FPPS model resulted in micropore Schulz distributions with a mean at $24.5 \AA$ with a variance of $4 \AA$ for the "difference" curve, and $10.9 \AA$ with a variance of $2 \AA$ for the contrast matched curve. For the 9.2\% burn-off sample, the "difference" curve resulted in a Schulz distribution with a mean at $18.2 \AA$ with variance of $4 \AA$, and $12.3 \AA$ with a variance of $2 \AA$ for the contrast matched curve. This increase in the "closed" mean micropore size, accompanied by a decrease in the "open" mean micropore size, from the unactivated sample to the $9.2 \%$ burn-off sample, is consistent with a propensity for preferential opening up of the smaller micropores first.

The contrast matched and "difference" curves for the high burn-off samples are presented in Figure 2.5-3. In this regime of behavior, all the "difference" curves lie above their corresponding contrast matched curves over the entire $q$ range. This indicates that most of the porosity over the entire size range is open. The contrast matched curves are quite similar to one another at low $\mathrm{q}$, indicating little difference in closed porosity. At high $\mathrm{q}$, however, there is an indication that closed microporosity is still being opened up between $32.7 \%$ and $58 \%$ burn-off.

The "difference" curves in Figure 2.5-3 first increase and then decrease. This corresponds quite well to the behavior of the BET surface areas of 270,397 , and $268 \mathrm{~m}^{2} / \mathrm{g}$ reported in Table $2.5-1$ for the $32.7 \%, 58 \%$, and $68 \%$ burn-off samples, respectively. The increase in open porosity over the entire pore size range suggests the creation of additional porosity, as well as pore widening due to 
gasification. At the highest burn-off of $68 \%$, the "difference" scattering decreases, indicating consumption of porosity over the entire size range, which is most marked in the micropore region. This latter effect is most probably due to pore wall collapse as a result of carbon consumption.

It was not possible to fit the scattering data for the "difference" curves in the high burn-off regime to a fractal/single mode FPPS model. It was possible, however, to identify two linear regions with a slope of -2.9 at low q, and -2.4 at high $q$ for the $32.7 \%$ burn-off sample. These correspond to scattering from mass fractals. Since fractal behavior is associated with wide pore size distributions, the picture that emerges is that of the activation considerably widening the original microporosity. That is, the fairly narrow Schultz distribution for the micropores in the low burn-off regime has been broadened into a fractal. Similar scattering behavior has been observed by Benedetti et al $^{50}$ for scattering from a partially crystalline zirconium oxide aerogel.

For the 58\% burn-off sample with the highest BET surface area, the "difference" curve is almost linear over the entire $q$ range with a slope of -2.6 , indicating that scattering is, therefore, from a mass fractal. The "difference" curve for the $68 \%$ burn-off sample also has two linear sections, similar to that for the $32.7 \%$ burn-off sample; one of slope -2.4 at low $q$, and the other of slope -3.3 at high $q$. The former is scattering from a mass fractal, and the latter is scattering from a surface fractal. This is consistent with the decrease in surface area between the $58 \%$ and $68 \%$ burn-off samples being due to the breakdown of the structure of the char as the carbon pore walls due to gasification.

The Porod invariants (calculated according to Eq. [1]) of the data presented in Figures 2.5-1-3 provide a compact representation of the behavior of all the scattering data. In Figure 2.5-4 are presented the Porod invariant values for all the data in the form of bar graphs. In this figure, the total heights of the bars represent the total scattering from the dry samples, and the relative contributions of the contrast matched and "difference" scattering intensities are indicated by the corresponding areas in each case. The data in Figures 2.5-1 and 2.5-4 indicate that the total scattering changes relatively little in the low burn-off regime. As shown in Figure 2.5-4, and as already discussed above, the contribution from the contrast matched scattering decreases at the expense of the "difference" scattering. These data are consistent with some previously closed or inaccessible porosity being opened up by gasification of carbon blocking this porosity. From Figure 2.5-2, it is noted that most of the porosity that has been opened up lies in the micropore region for $q>0.1 \AA^{-1}$.

Wyodak coal contains a significant amount of mineral matter $6.31 \%$ (as received) ${ }^{51}$. Following pyrolysis, the mineral matter content increases to about $15.9 \%$ on a dry basis. The principal constituents of the ash are alumina, iron oxide, calcium oxide, silica, and sulfite. It is expected that the contrast difference between these minerals and the carbon/deuterated toluene also contributes to the contrast matched scattering intensity. Therefore, it is assumed that the scattering intensity from the contrast matched samples results primarily from two sources: inaccessible porosity and mineral matter contrast differences. The data in Figure 2.5-4 indicate that the contrast matched scattering intensity decreases progressively and monotonically with burn-off up to $58 \%$ burn-off. If it is assumed that there is no residual inaccessible porosity by $58 \%$ burn-off, then the contrast matched contribution at this burn-off may be attributed almost completely to mineral matter contrast differences. From the Porod invariants for the $58 \%$ burn-off sample this means that $\sim 11 \%$ of the total scattering is due to mineral matter contrast differences. For the unactivated sample, this 
increases to $\sim 40 \%$. Consequently, the effects of mineral matter on neutron scattering intensities can be significant for coal chars, and thus should be taken into account in quantitative assessments.

An estimate of the original closed porosity can be obtained by subtraction of the $58 \%$ burn-off contrast matched curve in Figure 2.5-3, assuming it is mostly due to mineral matter contrast effects, from that of the unactivated sample in Figure 2.5-2. As may be expected, this yields a well-defined microporosity region at high $\mathrm{q}$, and a curve with a fractal dimension of 2.6 at low $q$.

2.5-4.2 Phenolic Resin Char. The burn-offs, BET surface areas, and Porod invariants of the four phenolic resin samples are presented in Table 2.5-2. The scattering intensity results from the four "dry" PRC are presented in Figure 2.5-5. Perhaps the most notable feature in this figure is that the total scattering decreases with increasing activation, except between the $12.6 \%$ and $21 \%$ bumoff samples, which are both quite similar. This differs from what is shown in Figure 1 for the Wyodak coal char, which is more typical behavior for carbons and chars, such as that indicated by the X-ray scattering from Carbosieve-S gasified in $\mathrm{CO}_{2}$, ${ }^{52}$ as discussed above.

It is well known that non-graphitizing carbons are generally composed of a mixture of ordered aromatic regions and disordered regions. It has been observed that during oxidation of carbon blacks and partially graphitized carbons, the disordered regions are the most reactive and are attacked most rapidly..$^{53}$ This behavior also applies to PRC. It has been reported that the crystallite dimensions of the ordered regions in a $1000^{\circ} \mathrm{C}$ PRC did not change appreciably up to $10 \%$ burnoff; and up to $40 \%$ burn-off for a $1400^{\circ} \mathrm{C} \mathrm{PRC} .^{54}$ This strongly implies that the burn-off must have proceeded primarily in the disordered carbon regions. It has been hypothesized that the disordered regions arise primarily from the methylene bridge carbons in the phenolic resin precursor, according to studies employing ${ }^{13} \mathrm{C}$-labeling of the resin via the formaldehyde reagent ..$^{55,56}$

As demonstrated below, in the current work the primary mechanism of porosity development at low burn-off in the PRC samples is via the opening of "closed" or inaccessible porosity, as opposed to the development of new porosity. The mechanism of opening the "closed" porosity is by gasification of carbon that initially "blocks" the intrinsic porosity. The preferential nature of the gasification suggests that this carbon is primarily disordered. It is also reasonable to assume that much of the interfacial scattering surface between the carbon and the voids (i.e., the porosity) is composed of carbon crystallites that typically form the walls of the "slitlike" micropores that constitute a large fraction of the inherent porosity. This model predicts that total scattering will decrease with burn-off if the removal of carbon does not appreciably alter the existing pore structure. This hypothesis is believed to account for the behavior evident in Figure 2.5-5.

The contrast matched spectra corresponding to the samples in Figure 2.5-5 are presented in Figure 2.5-6. As shown, the $0 \%$ and $1.5 \%$ burn-off samples exhibit very similar, high scattering intensities; while the $12.6 \%$ and $21 \%$ burn-off samples exhibit very similar, but significantly lower scattering intensities. This is consistent with a considerable loss of inaccessible porosity between $1.5 \%$ and $12.6 \%$ burn-off, as it is opened and converted to accessible porosity. This trend is also evident in the "difference" scattering intensities presented in Figure 2.5-7, which are indicative of the "open" or accessible porosity. As shown, the $0 \%$ and $1.5 \%$ burn-off samples exhibit very low "difference" scattering intensities, especially in the micropore size region; while the $12.6 \%$ and $21 \%$ burn-off samples exhibit very similar, high "difference" scattering intensities, especially in 
the micropore region. Moreover, it is important to note that although the scattering intensity of the $1.5 \%$ burn-off sample is less than for the unactivated sample over the entire q-range, it appears that this decrease is much more marked in the "difference" (i.e., Figure 2.5-7) than in the contrast matched (i.e., Figure 2.5-6) spectra in the region of larger scatterers. This is consistent with the hypothesis that the consumption of disordered carbon is causing the decrease in scattering, since this must occur primarily via the accessible (i.e., "difference"), rather than the inaccessible (i.e., contrast matched) porosity.

The Porod invariants of the data presented in Figures 2.5-5, 6, and 7, are presented in the form of bar graphs in Figure 2.5-8, as was done previously for the Wyodak coal char scattering results. As shown, these values reveal the same qualitative behavior discussed above. That is, the bar graphs indicate that total scattering decreases the most initially, and the relative contribution from contrast matched scattering, which is indicative of the "closed" or inaccessible porosity, decreases precipitously with burn-off, while the "difference" scattering exhibits decidedly inverse behavior. These data are also consistent with the bulk of the porosity being present initially as "blind" or inaccessible porosity that is "opened" up by gasification, such that the majority of the inaccessible porosity has been converted to accessible somewhere between $1.5 \%$ and $12.6 \%$ burn-off.

In Figure 2.5-9 are presented differences in total scattering intensities between the unactivated $(0 \%$ burn-off) sample and those that have been burned-off, $\Delta(0 \%-\mathrm{x} \%)$. These curves should be representative of the carbon that was burned-off up until that point. As shown, for all three burnoffs most of the scattering arises from larger scatterers in the $50 \AA-200 \AA$ size range. The $\Delta(0 \%$ 12.6\%) is reproduced in Figure 2.5-10. As shown, a unimodal FPPS Schulz distribution with a mean radius of $51 \AA$ and $b=1.01$ fits the data very well. The corresponding Schulz distribution of scatterers is reproduced in Figure 11, with a mean scatterer size of $102 \AA$. As shown in Figure 10 , a power-law fit to the same data is not as good as the unimodal Schulz distribution fit, but it produces a fractal dimension of 2.6 , which is the same as that found for all the contrast matched scattering. This suggests that the carbon that has been burned off is more convoluted or disordered than that which is left behind, and that it initially exists in association with the inaccessible porosity, and is preferentially burned off as the inaccessible porosity is opened up. It is most probably this carbon that initially "blocks" the inaccessible porosity.

A corollary of the preceding hypothesis is that the intrinsic underlying porosity which is opened up with increasing burn-off should be approximately represented by the total scattering curve from the dry $12.6 \%$ burn-off sample, from which much of the contribution from disordered carbon has disappeared. Another independent test of this conclusion is that the contrast matched scattering curve for the unactivated sample should also be representative of the same porosity since hardly any of the underlying porosity is accessible in the unactivated sample. As shown in Figure 2.5-8, the two curves are indeed practically the same, except for a slight discrepancy at low q. Consequently, the hypothesis is consistent with these data as well. The slight difference at low $q$ values is attributed to larger scatterers associated with the disordered carbon, which do not contribute to the scattering from the contrast matched unactivated sample presumably because most of the interfaces with this material are largely in contact with the contrast matching perdeuterated toluene. It is reasonable to assume that the smaller scatterers in the disordered carbon will react more rapidly than the larger ones, which would then account for the remaining large scatterers at $12.6 \%$ burn-off, as indicated by the data in Figure 2.5-9. The resultant fit to the contrast matched scattering intensity 
curve for the unactivated sample (CM-0\%) using a bimodal FPPS Schulz distribution is also presented in Figure 2.5-12. The corresponding bimodal Schulz distribution is presented in Figure 2.5-13. As shown, the underlying intrinsic porosity of the PRC is well described by a narrow distribution of micropores $\left(\mathrm{a}_{01}=4.7 \AA, \mathrm{b}_{1}=23\right)$, superimposed on a much wider distribution of scatterers $\left(\mathrm{a}_{02}=7.0 \AA, \mathrm{b}_{2}=0.082\right)$.

The $\Delta$ curves for the $12.6 \%$ and $21 \%$ burn-off are very similar over the entire $q$ range. The data also indicate ( $c f$. Figures 2.5-7 and 8) that these two samples still exhibit significant, although reduced, inaccessible porosity. Differences between the closed and open porosity for these two burn-offs are presented in Figure 2.5-14. As shown, scattering from the accessible porosity for the $12.6 \%$ burnoff sample is slightly greater than that from the $21 \%$ burn-off sample, and the reverse is true regarding the inaccessible porosity. It is also noted that the nature of these differences in scattering is very similar to that caused by the disordered carbon, which is also plotted in Figure 2.5-14 for comparison. Therefore, it is unlikely that new porosity is being developed yet in these samples. It is also noted that it is primarily the very largest, accessible scatterers that disappear between $12.6 \%$ and $21 \%$ burn-off, while it is intermediate size scatterers that appear in the inaccessible spectra for the $21 \%$ burn-off sample. One possible explanation of this behavior is that at this stage of burn-off it is primarily the largest scatterers from the disordered carbon that remain, and that continued oxygen attack not only consumes them, but also results in their fragmentation. The resultant fragments would be smaller in size and the new scattering interfaces that are created by this process apparently are not all accessible to the perdeuterated toluene until (presumably) more burn-off occurs. As noted previously, that the larger scatterers tend to survive longer than the smaller scatterers is supported by the trend in the "difference" curves at the very lowest q values in Figure 2.5-9.

In a study on the structural properties of a phenol-formaldehyde resin char, Román-Martínez et al. ${ }^{57}$ observed by SEM, globular structures made up of graphitic planes with a large number of defects. These structures varied in size between 100 and $300 \AA$, which is quite similar to the size range indicated by the disordered carbon distribution presented in Figure 7. In another study, Gupta and Harrison [30] found that during carbonization of phenolic resins at $723 \mathrm{~K}$ and $1023 \mathrm{~K}$, the destruction of crosslinks leads to the formation of clusters of aromatic units. The latter are probably the same as the globular structures observed by Román-Martínez et al. ${ }^{58}$ Gupta and Harrison ${ }^{59}$ also found that their char was conductive, suggesting the presence of large ordered graphitic planes as well. These observations are completely consistent with the hypothesis formulated above that the PRC consists of both disordered (i.e., globular structures) and ordered (i.e., large graphitic layers), and that the burn-off of the larger disordered carbon structures opens up the blind porosity which they originally "block."

The data from the unactivated contrast matched sample (i.e., approximately equivalent to the intrinsic porosity) were also fit to the fractal-unimodal FPPS model. The resultant mean micropore size was $9.1 \AA$, which is in very good agreement with the value of $9.4 \AA$ obtained with the bimodal FPPS Schulz model for the smaller pores, as presented in Figure 13. A summary of the resultant fractal dimensions from the power law fits at low $q$ values for all the data is presented in Table 3 . These values reveal an interesting pattern that is consistent with the preceding interpretation of the scattering data. The unactivated sample exhibits a constant high fractal dimension (i.e., surface roughness) for both the accessible and inaccessible porosity. This is consistent with disordered 
material initially being associated with practically all the intrinsic porosity throughout the sample; i.e., essentially all the intrinsic porosity is initially "blocked." For the three activated samples, however, the fractal dimension of the accessible ("difference") porosity is reduced to 2.2 , while that for the inaccessible porosity remains at the initial value of 2.6, just as for the unactivated sample. This suggests that the accessible porosity has been rendered somehow smoother, most probably due to consumption of disordered material.

2.5-5. Summary \& Conclusions. It has been shown that the analysis of small angle neutron scattering data employing contrast matching can provide a considerable amount of information concerning the details of porosity development, activation mechanisms, and the nature of carbon materials. This was demonstrated with two significantly different carbons.

In the case of Wyodak coal char, it was shown that total scattering generally increased with activation. The unactivated sample exhibited considerable closed porosity, especially in the micropore size range at high q. Results at increasing levels of activation show the contributions from unblocking this closed porosity and developing new porosity. Finally, the effects of pore wall collapse are quite evident in the high burn-off data. In addition, contrast matching was able to yield an estimate of the relative effects of mineral matter on the scattering intensities.

The activation behavior of the phenolic resin char was different. Unlike other carbons which are activated primarily via developing new porosity and widening existing porosity with increasing burn-off, the glassy carbon evolves during initial burn-off primarily by preferential consumption of disordered carbon which initially "blocks" the underlying intrinsic porosity. The nature of this intrinsic porosity does not appear to be affected very much in this regime of behavior, other than being progressively exposed by this process. The disordered carbon material that is removed by reaction appears to exhibit a broad range of sizes with a mean scatterer size of about $102 \AA$. This is only an estimate, however, since it seems that the smaller scatterers in the disordered carbon material are consumed before the larger ones. The distribution of the underlying, intrinsic porosity was found to be well fit by a bimodal Schulz distribution . of fully penetrable polydisperse spheres (FPPS), comprised of a narrow distribution in the micropore size range with a mean of about $9.4 \AA$, superimposed on another broad distribution of pore sizes. The primary effects of burn-off on this carbon material are progressive removal of disordered carbon, resulting in the conversion of initially inaccessible to accessible porosity. There was no evidence of significant widening or alteration of the intrinsic underlying porosity, or of the development of new porosity up to $21 \%$ burn-off. 
Table 2.5-1. BET surface areas and Porod invariants for Wyodak coal char samples.

\begin{tabular}{|c|c|c|c|c|}
\hline Burn-off (\%) & $\begin{array}{c}\text { BET Surface Area } \\
\left(\mathrm{m}^{2} / \mathrm{g}\right)\end{array}$ & \multicolumn{3}{|c|}{ Porod Invariant } \\
& & $\mathbf{x ~ 1 0 ^ { 1 1 } \AA ^ { - 4 } )}$ \\
\cline { 2 - 5 } & 12 & Dry & $\mathbf{M}$ & Difference \\
\hline 0 & - & 3.13 & 2.12 & 1.01 \\
\hline 7.4 & 140 & 3.10 & 1.60 & 1.50 \\
\hline 9.2 & 270 & 3.35 & 1.64 & 1.71 \\
\hline 33.7 & 397 & 5.16 & 1.48 & 3.68 \\
\hline 58 & 268 & 11.58 & 1.25 & 10.33 \\
\hline 68 & & 6.16 & 1.32 & 4.84 \\
\hline
\end{tabular}

Table 2.5-2. BET surface areas and Porod invariants for phenolic resin char samples.

\begin{tabular}{|c|c|c|c|c|}
\hline Burn-off (\%) & $\begin{array}{c}\text { BET Surface Area } \\
\left(\mathrm{m}^{2} / \mathrm{g}\right)\end{array}$ & \multicolumn{3}{|c|}{$\begin{array}{c}\text { Porod Invariant } \\
\left(\times \mathbf{1 0}^{11} \AA^{-4}\right)\end{array}$} \\
\cline { 3 - 5 } & & Dry & C-M & Difference \\
\hline 0 & 2.2 & 2.15 & 1.52 & 0.63 \\
\hline 1.5 & 5.0 & 1.89 & 1.34 & 0.55 \\
\hline 13 & 15.7 & 1.86 & 0.60 & 1.26 \\
\hline 21 & 27.7 & 1.75 & 0.67 & 1.08 \\
\hline
\end{tabular}

Table 2.5-3. Fractal dimensions at low $q$ for phenolic resin char samples.

\begin{tabular}{|c|c|c|c|c|}
\hline Burn-off (\%) & $\begin{array}{c}\text { BET Surface Area } \\
\left(\mathrm{m}^{2} / \mathrm{g}\right)\end{array}$ & \multicolumn{3}{|c|}{ Fractal Dimension, D } \\
\cline { 3 - 5 } & & Dry & C-M & Difference \\
\hline 0 & 2.2 & 2.6 & 2.6 & 2.5 \\
\hline 1.5 & 5.0 & 2.6 & 2.6 & 2.2 \\
\hline 13 & 15.7 & 2.5 & 2.6 & 2.2 \\
\hline 21 & 27.7 & 2.4 & 2.6 & 2.2 \\
\hline
\end{tabular}




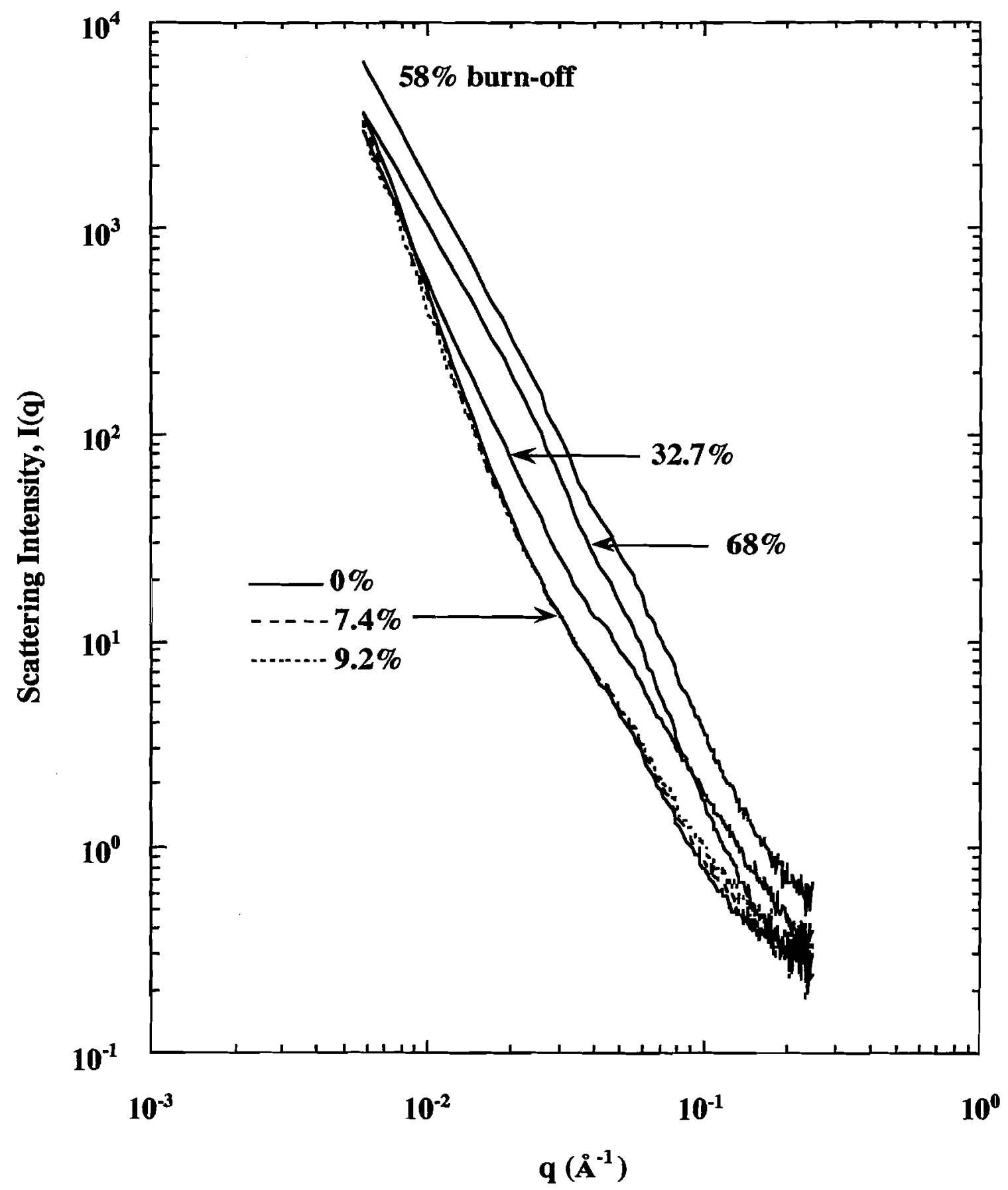

Figure 2.5-1. Small angle neutron scattering intensities from "dry" samples of Wyodak coal char as a function of extent of activation. 


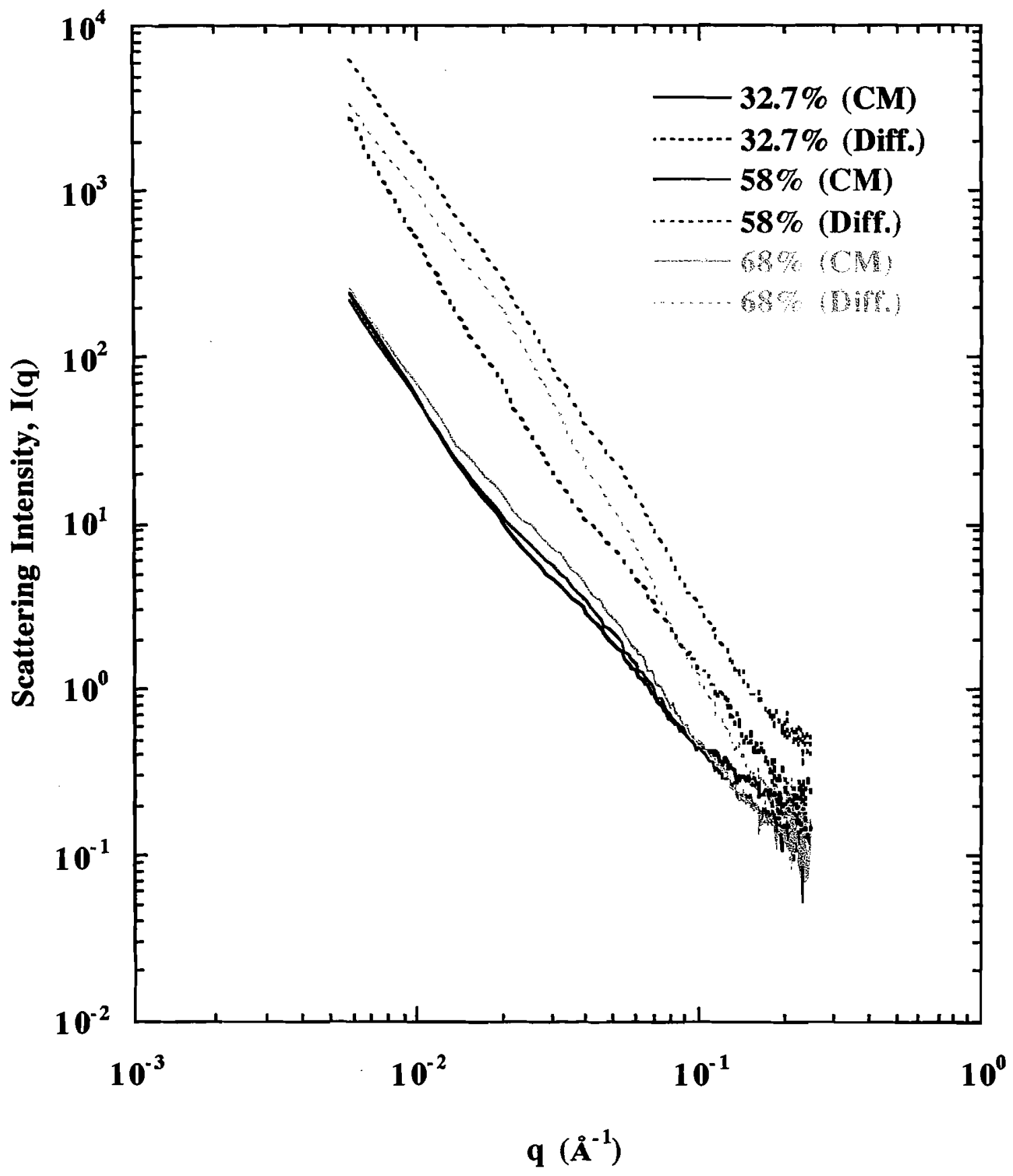

Figure 2.5-2. Small angle neutron scattering intensities from the low bum-off contrast matched (i.e., with perdeuterated toluene) samples and their corresponding "difference" scattering intensity curves. 


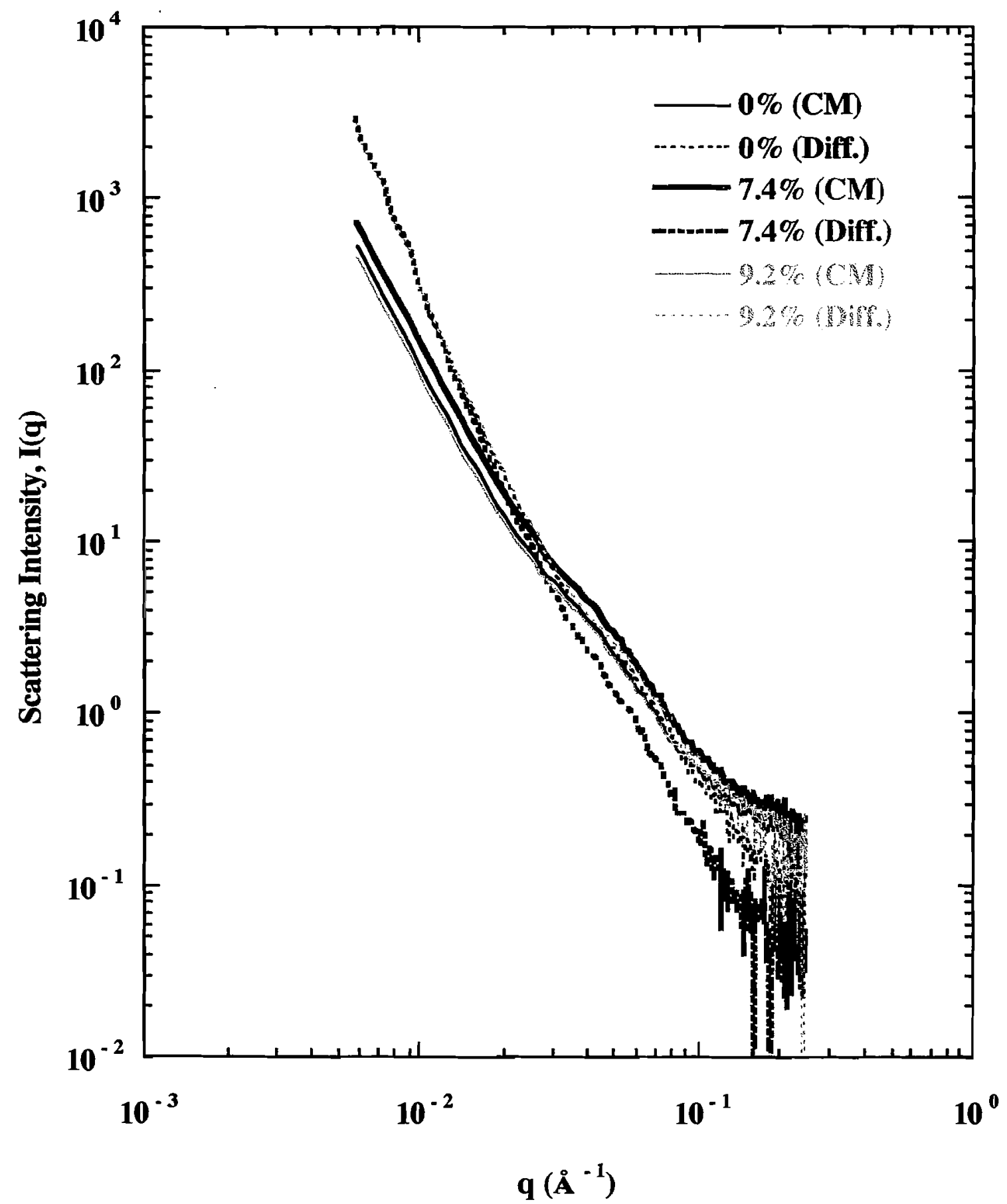

Figure 2.5-3. Small angle neutron scattering intensities from the high burn-off contrast matched (i.e., with perdeuterated toluene) samples and their corresponding "difference" scattering intensity curves. 


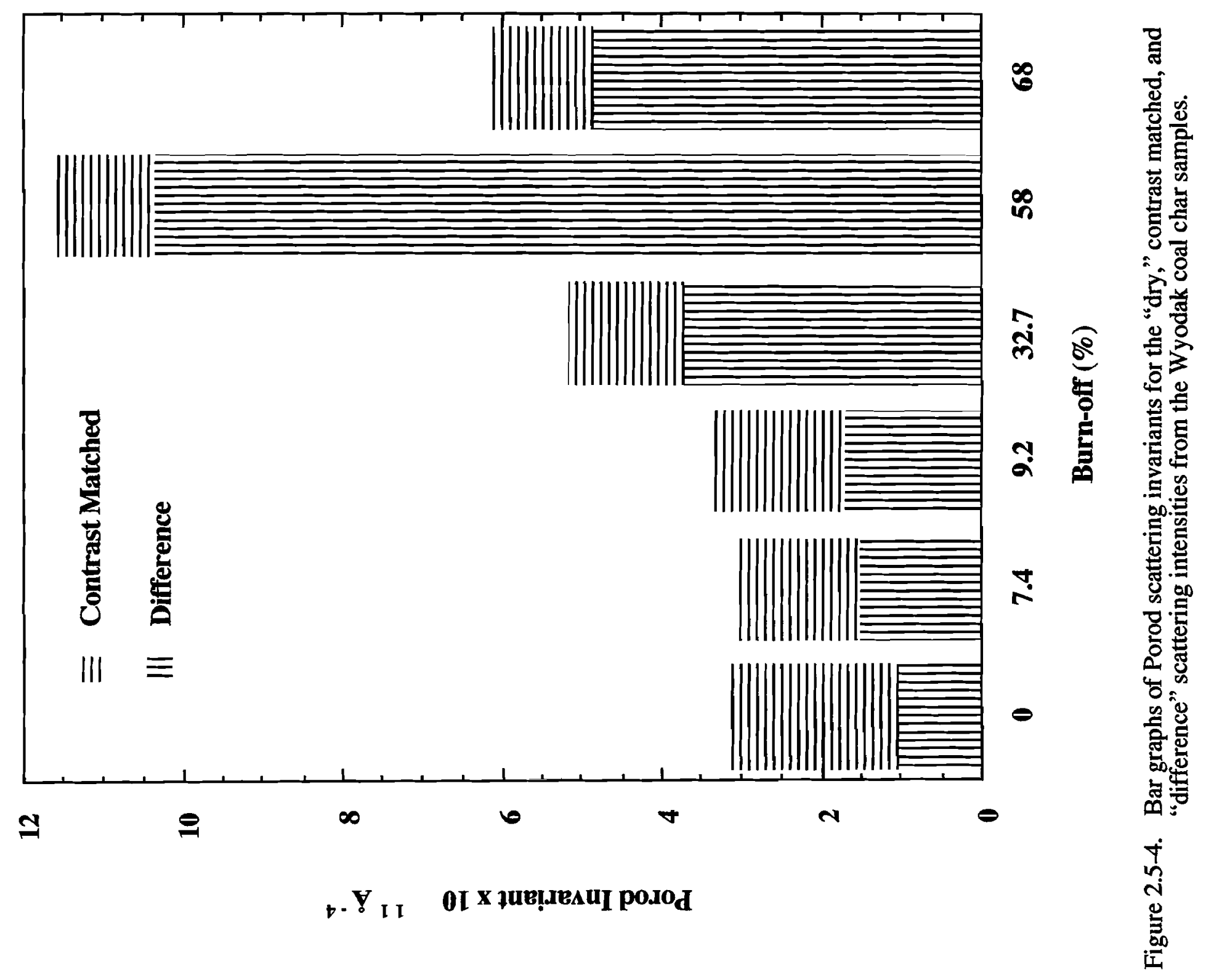




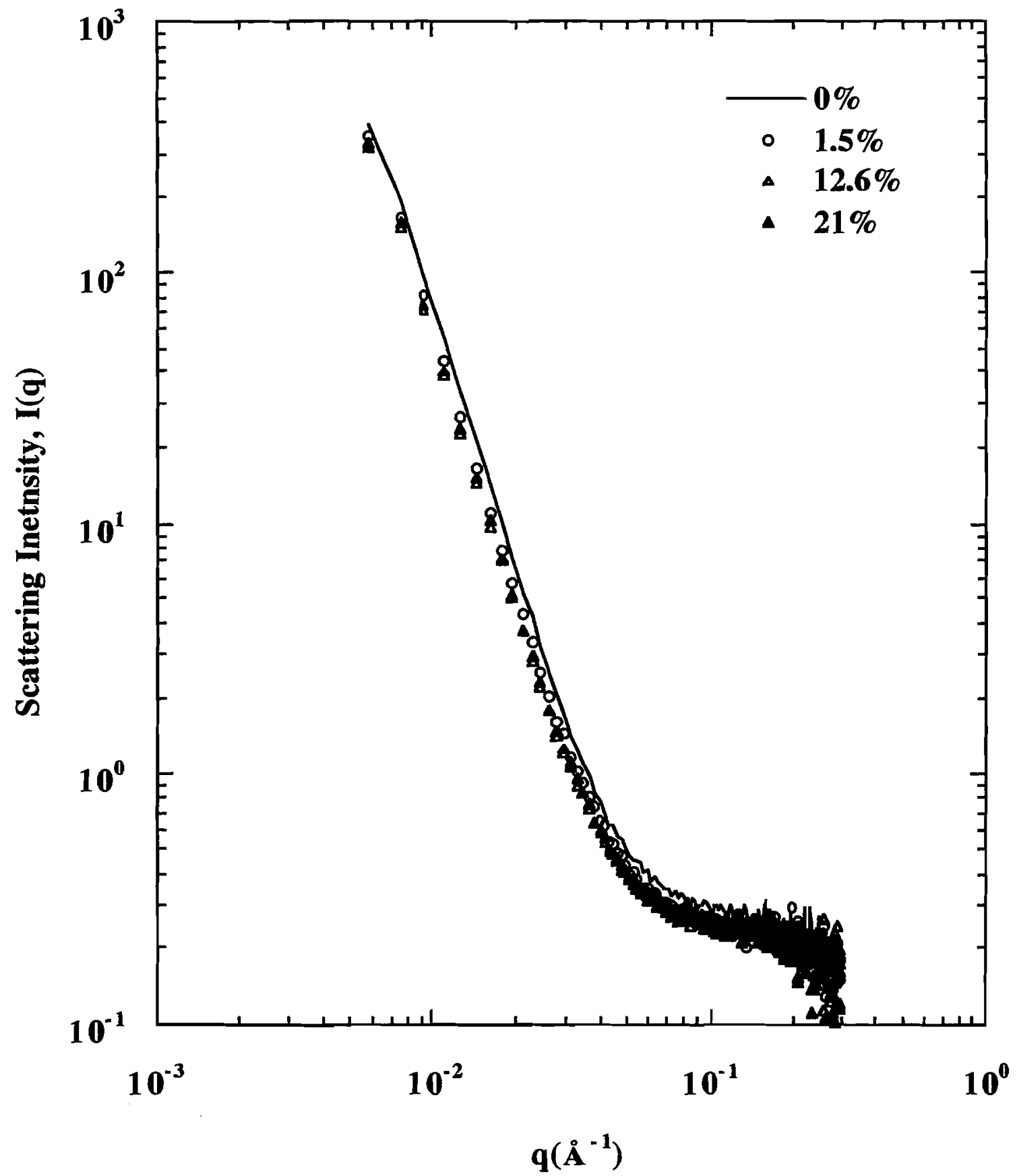

Figure 2.5-5. Small angle neutron scattering intensities from "dry" samples of phenolic resin char as a function of extent of activation. 


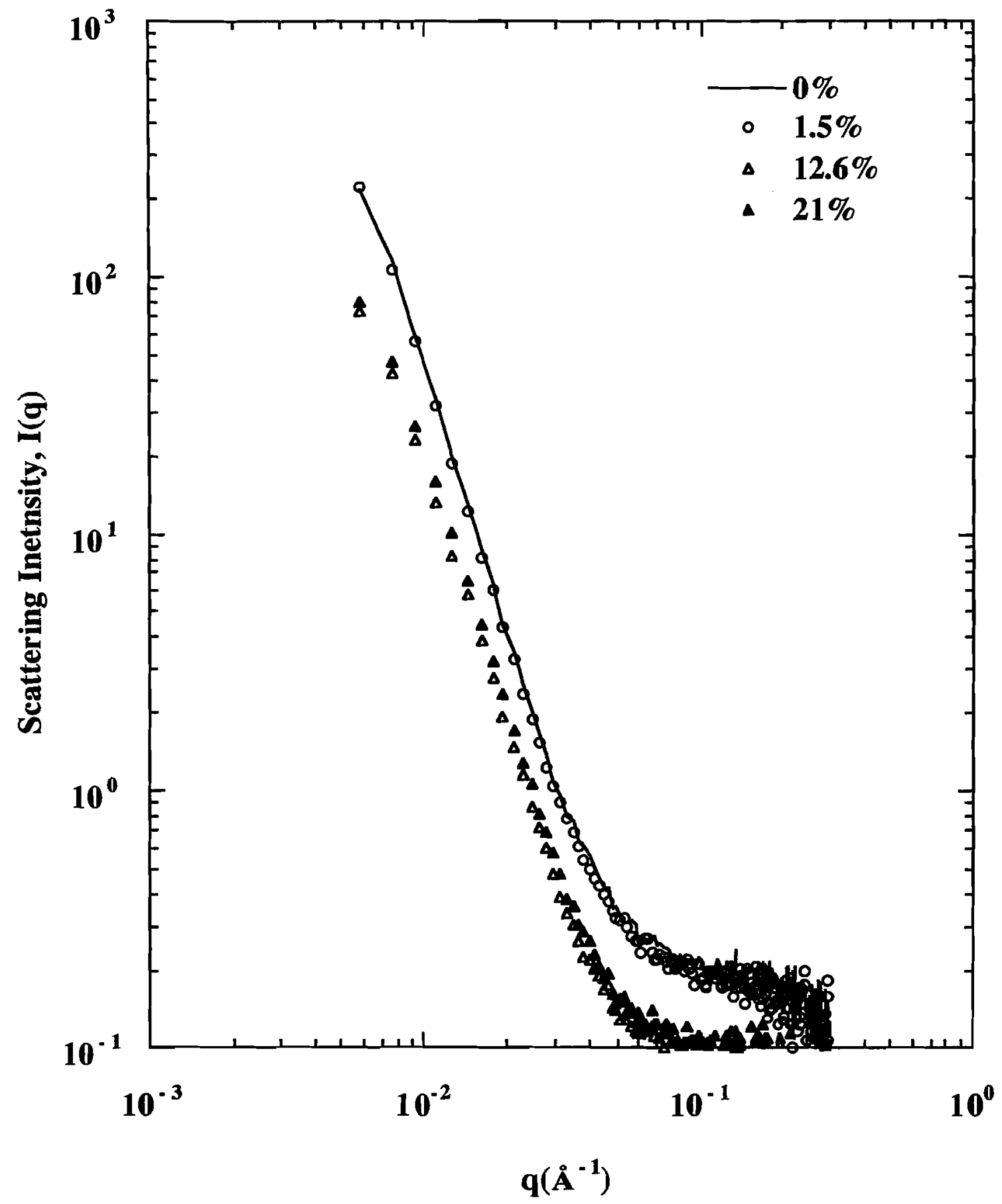

Figure 2.5-6. Small angle neutron scattering intensities from contrast matched (i.e., with perdeuterated toluene) samples corresponding to those in Figure 2.5-5. 


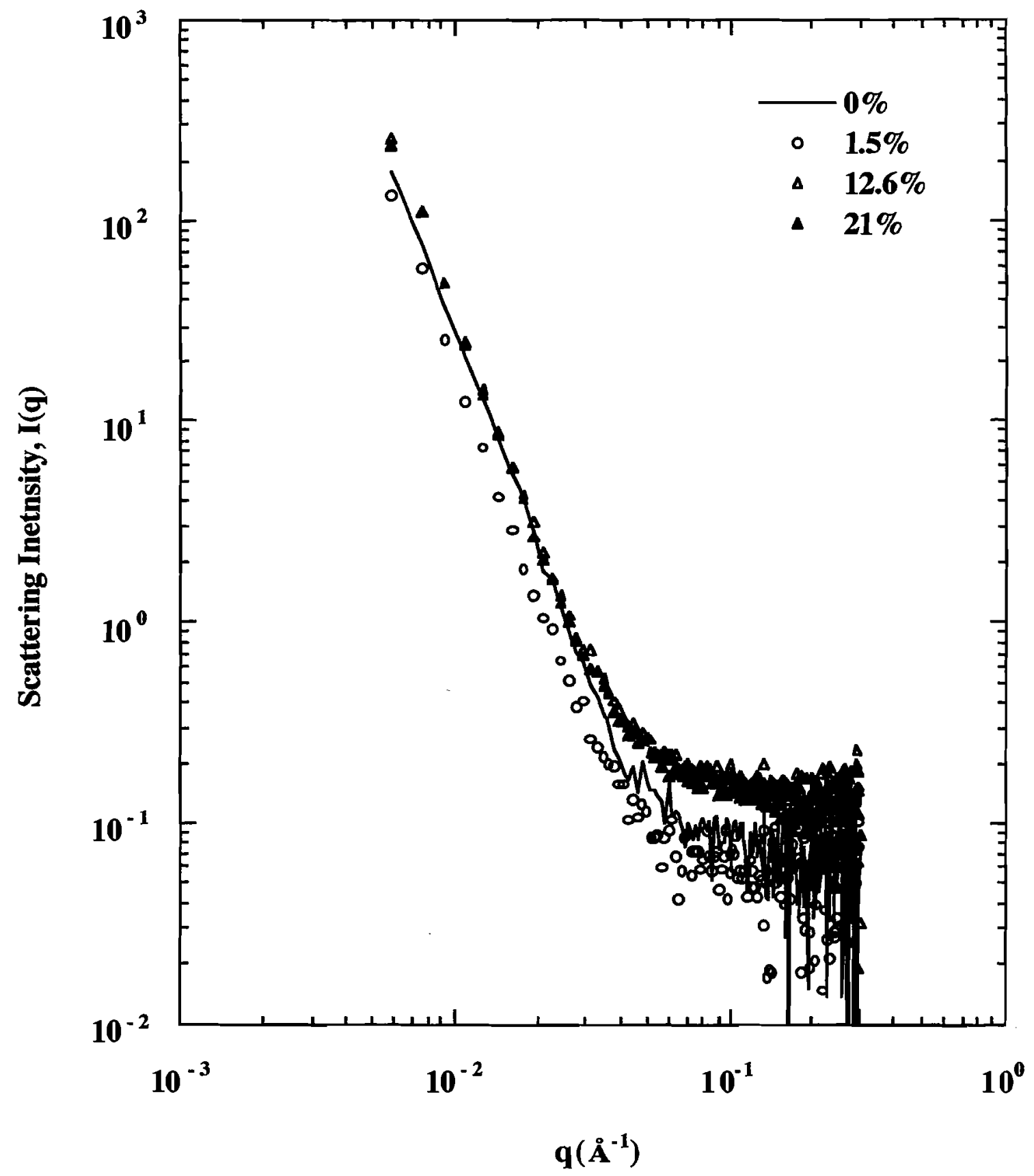

Figure 2.5-7. "Difference" spectra determined by the difference between the corresponding scattering curves presented in Figures 5 and 6 (i.e., "dry" - contrast matched). 


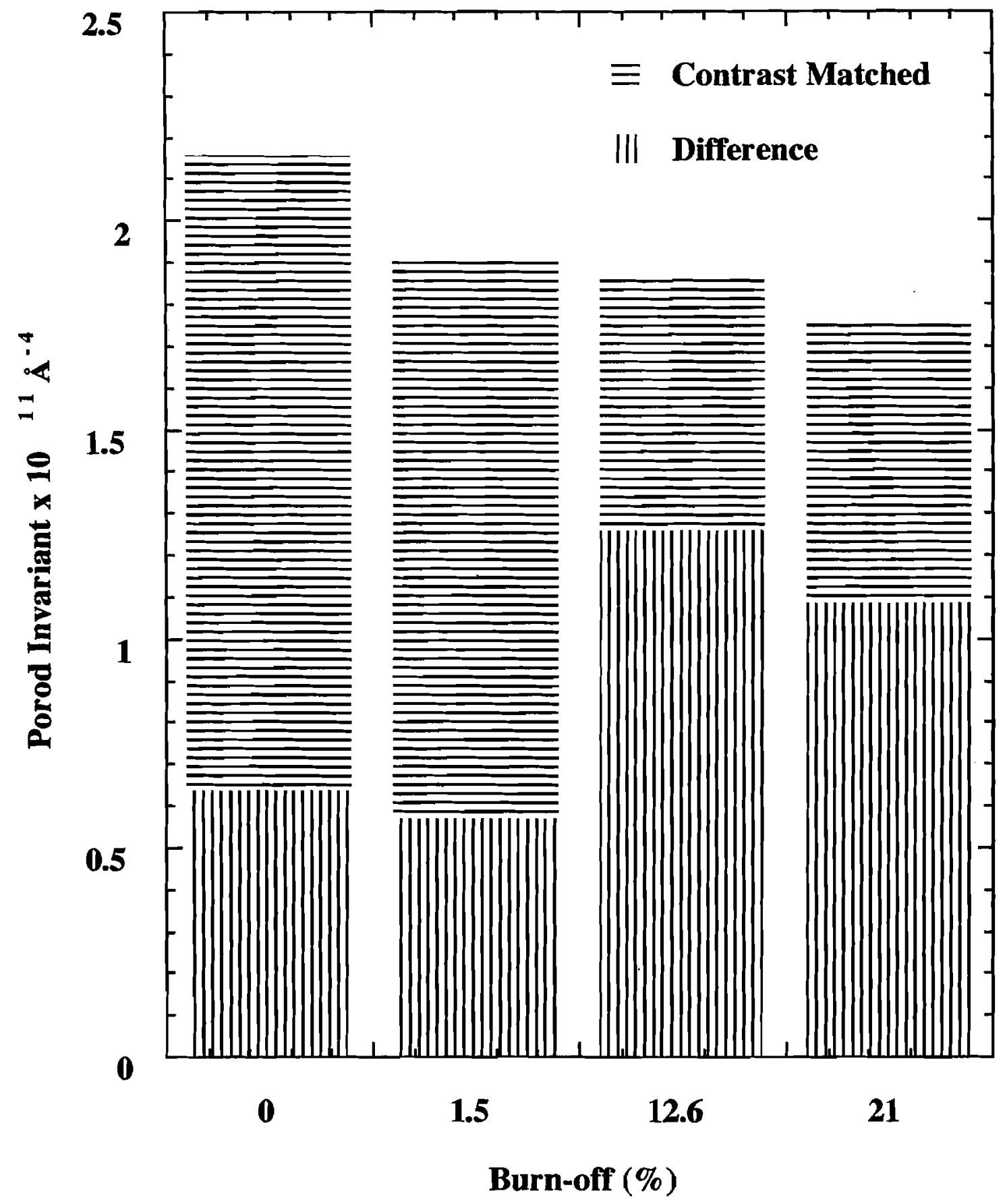

Figure 2.5-8. Bar graphs of Porod scattering invariants for the "dry," contrast matched, and "difference" scattering intensities from the phenolic resin char samples. 


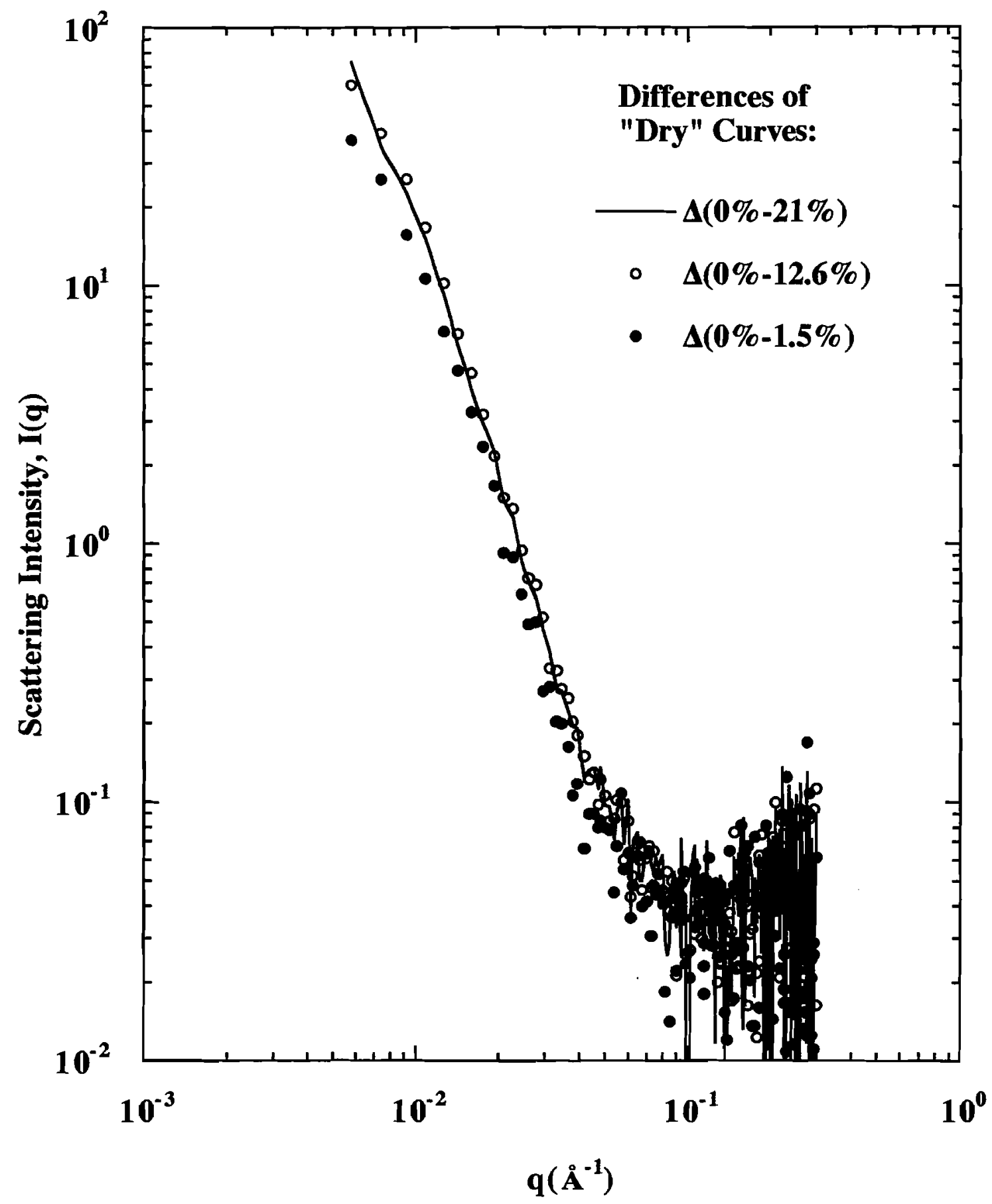

Figure 2.5-9. Differences in total scattering intensities between the unactivated sample and the three burned-off samples, $\Delta(0 \%$-x\%). The resultant scattering intensities are due to the burned-off carbon. 


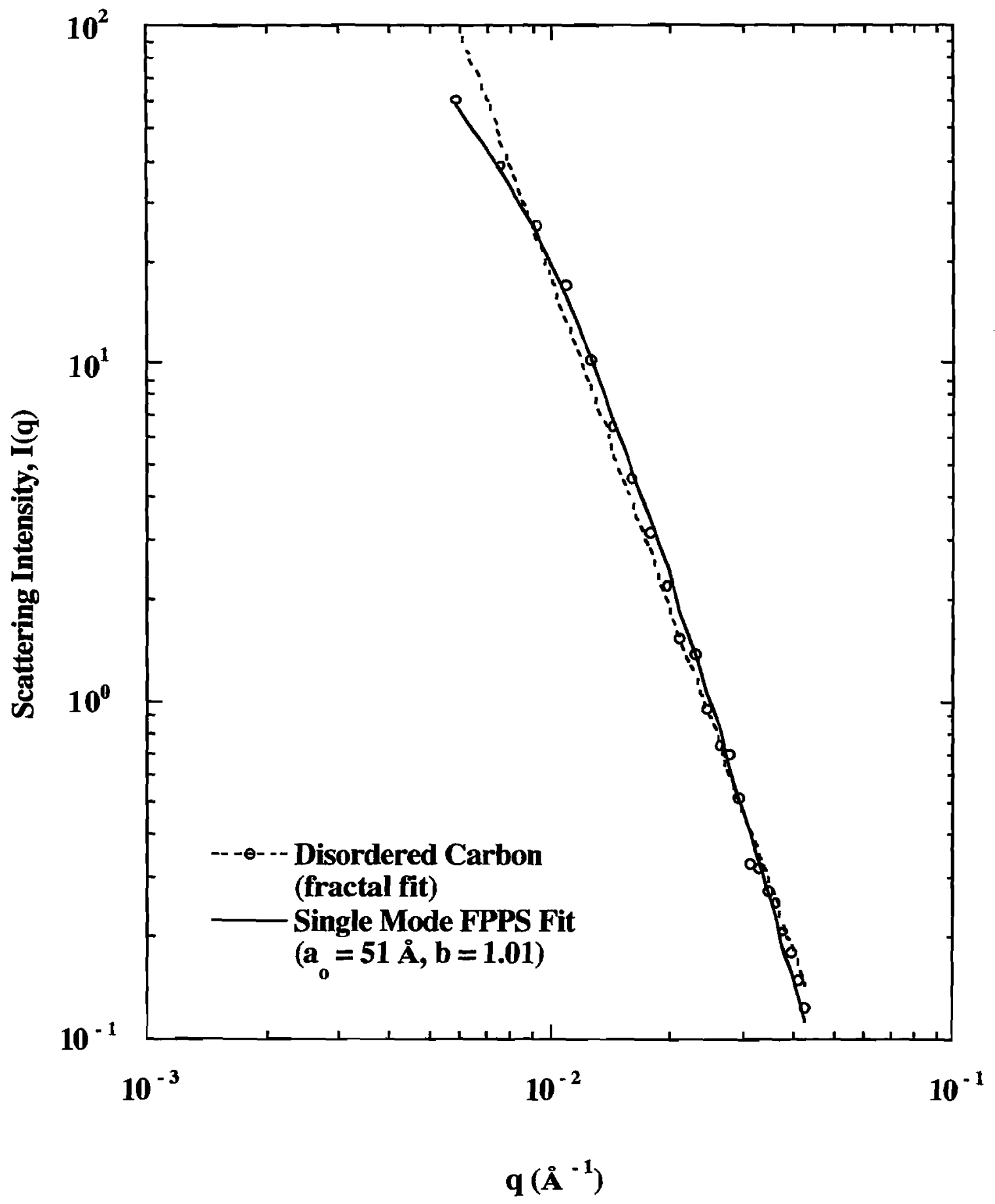

Figure 2.5-10. Distribution of disordered carbon burned-off to $12.6 \% ; \Delta(0 \%-12.6 \%)$. The curve fit is a FPPS unimodal Schulz distribution with a mean radius of $51 \AA$ and $b=1.01$. 


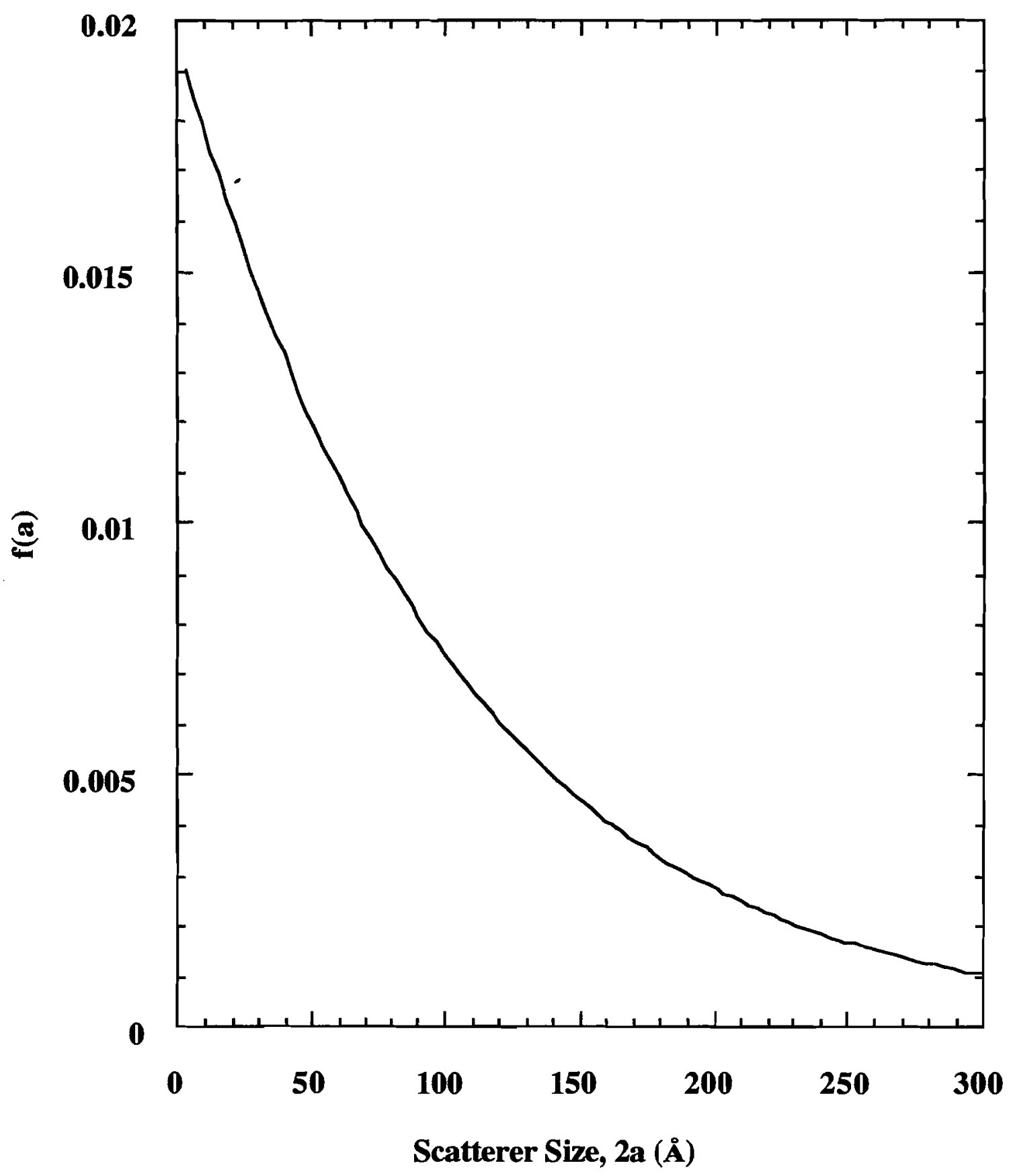

Figure 2.5-11. FPPS unimodal Schulz distribution for the scattering intensity fit presented in Figure $10\left(\mathrm{a}_{\mathrm{o}}=51 \AA, \mathrm{b}=1.01\right)$. 


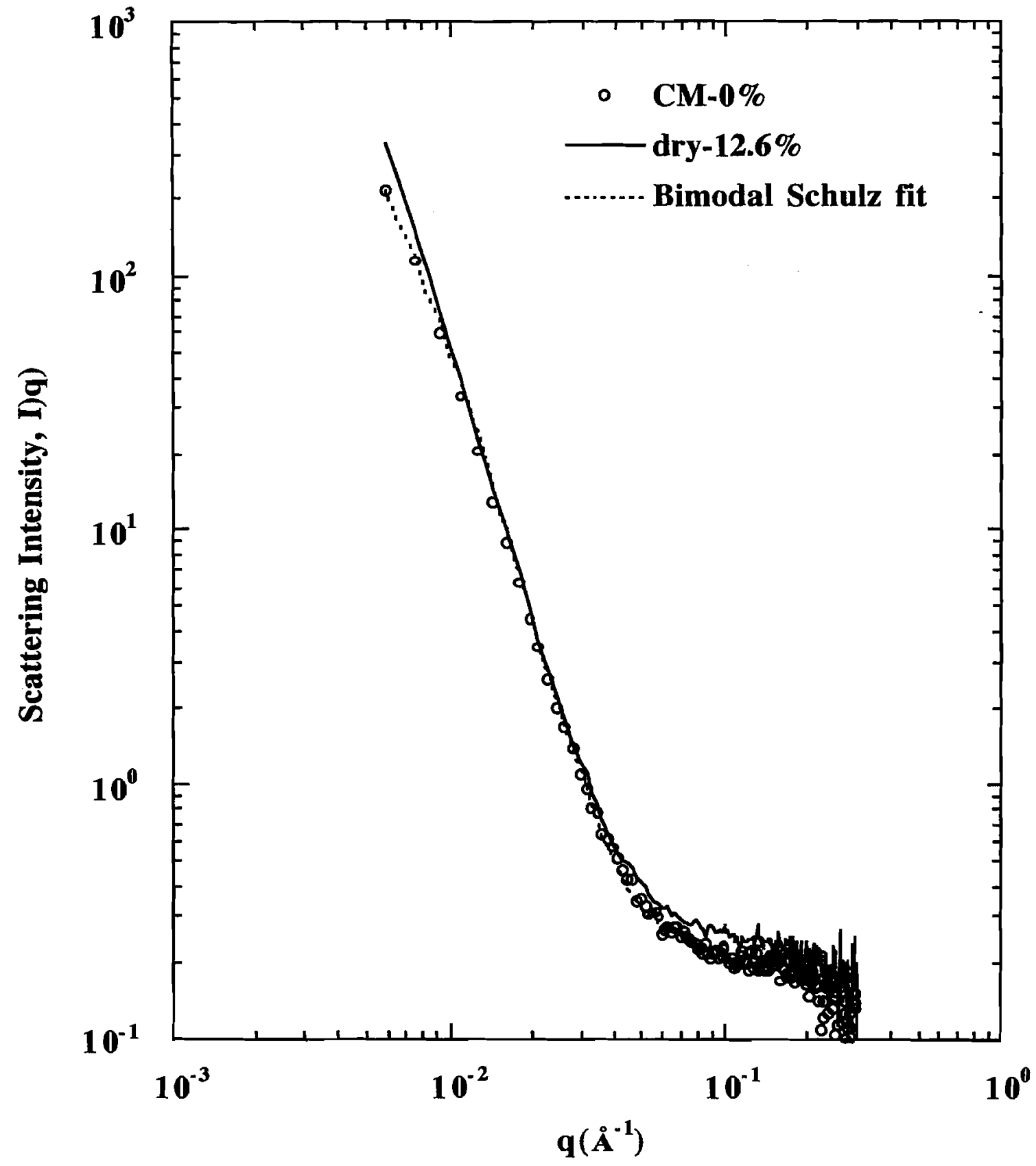

Figure 2.5-12. Distribution of the intrinsic underlying porosity from: (a) the dry $12.6 \%$ burn-off sample; and (b) the contrast matched scattering curve for the unactivated sample. The fit to the latter data was obtained using a FPPS bimodal Schulz distribution. 


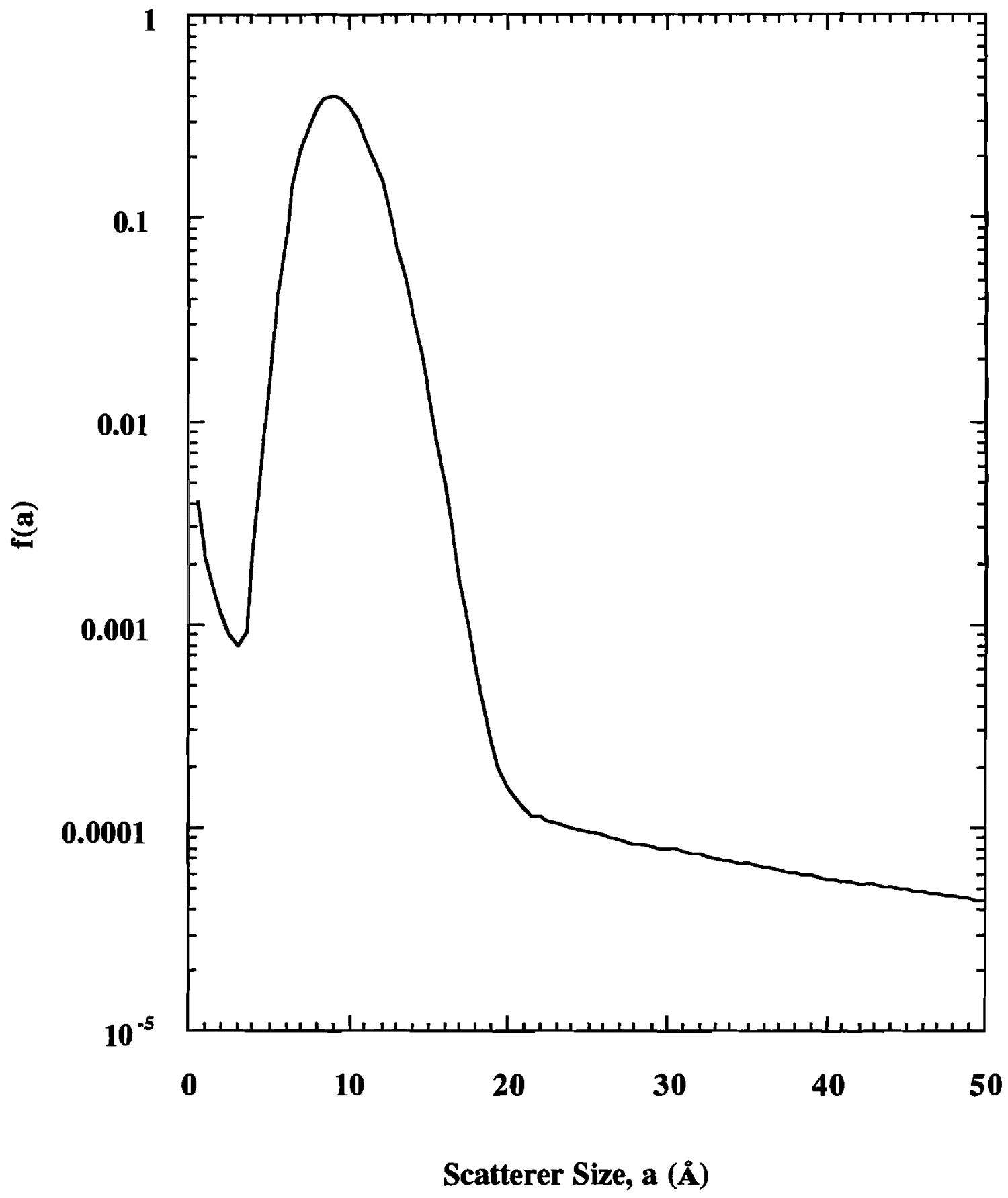

Figure 2.5-13. FPPS bimodal Schulz distribution used to fit the scattering intensity data in Figure 12: $\mathrm{a}_{01}=4.7 \AA, \mathrm{b}_{1}=23 ;$ and $\mathrm{a}_{02}=7.0 \AA, \mathrm{b}_{2}=0.082$. 


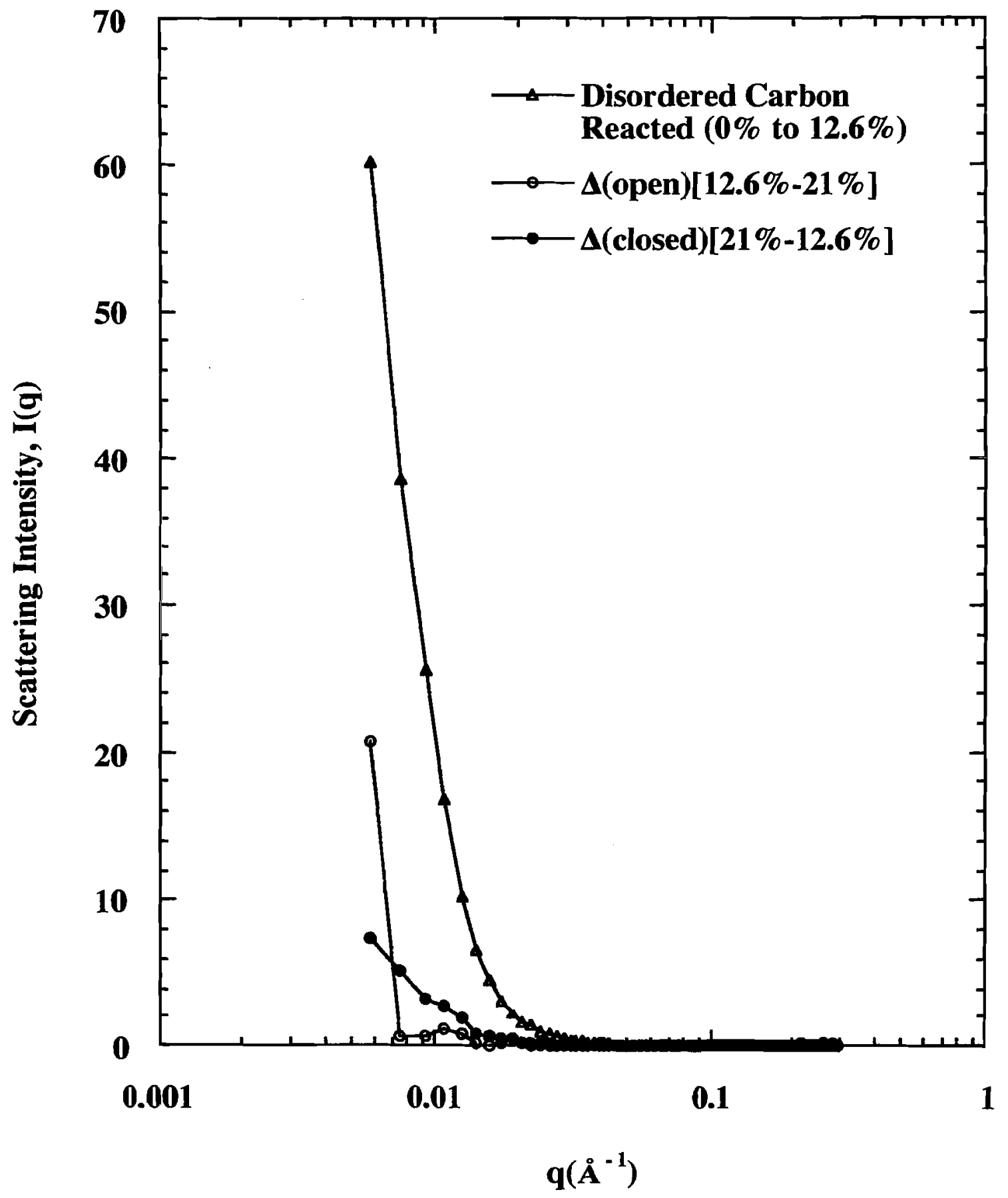

Figure 2.5-14. Differences between the "closed" and "open" porosity for the $12.6 \%$ and $21 \%$ burn-off samples, as well as the disordered carbon distribution from Figure 2.5-10. 


\section{References for Sections 2-1 through 2.5}

Wójtowicz, M., Ph.D. Dissertation, 1988.

2 Shim, H.-S., and R.H. Hurt, Proc. 23rd Biennial Conf. Carbon, The Pennsylvania State University, 1997, p. 438.

3 Kang, S.G., A.F. Sarofim, and J.M. Beer, Twenty Fourth Symp. (Int.) Combustion, The Combustion Institute, Pittsburgh, PA, 1992, pp. 1153-1159.

4 Srinivasachar, S., Senior, C.L., Helble, J.J., and Moore, J.W., Twenty Fourth Symp. (Int.) Combustion, Combustion Institute, Pittsburgh, PA, 1992, pp.1179-1187.

5 Lunden, M., C. Shaddix, D. Hardesty, N. Yang, and T. Headley, "Mineral Matter Effects on Char Structural Evolution and Oxidation Kinetics During Coal Char Combustion," WSS/CI97S-074, Sandia National Laboratories, Livermore, CA, 1997.

6 Morgan, M.E., Jenkins, R.G., and Walker, P.L., Jr. Fuel 60, 189, 1981.

7 Hachmann, L., Burmett, A., Gao, Y.-M., Hurt, R.H., and Suuberg, E.M., Proc. Twenty Seventh Symp. (Int.) Combustion, Pittsburgh, PA, 1998.

8 Gupta, A. and Harrison, I.R., Carbon, 1994, 32, 953.

9 Yamashita, Y. and Ouchi, K., Carbon, 1981, $19,89$.

10 Miura, C. and Low, M.J.D., Carbon, 1985, 23, 525.

$"$ Roman-Martinez, M.C., Cazorla-Amoros, D., Linares-Solano, A., Salinas-Martinez de Lecea, C. and Atamny, F., Carbon, 1996, 34, 719.

12 Chang, H.W. and Rhee, S.K., Carbon, 1978, $16,17$.

13 Zhang, L., Ph.D. Dissertation. Brown University, 1996.

14 Hurt, R.H., Dudek, D.R., Longwell, J.P., and Sarofim, A.F., Carbon 26(4), 433, 1988.

15 Waters, B.J., R.G. Squires, N.M. Laurendeau, and R.E. Mitchell, Comb. Flame 74, 91, 1988.

16 J.M. Calo and P.J. Hall, in Fundamental Issues in the Control of Carbon Gasification Reactivity, J. Lahaye and P. Ehrburger, Eds., NATO ASI Series, Series E: Applied Sciences, Vol. 192, Kluwer Academic Publishers, Dordrecht, 1991, p. 329.

17 Donnet, J.B., Carbon, 1982, $20,267$.

18 Senneca, O., Salatino, P., and Masi, S., Proc. Carbon '97, 1997, p. 396.

19 Lowell, S. and Shields, J.E., in Powder Surface Area and Porosity, Chapman \& Hall, London, 1984.

20 P.J. Hall, Chem. Phys. Lett. 124 (1986) 467.

21 G. Zgrablich, S. Mendioroz, L. Daza, J. Pajares, V. Mayagoitia, F. Rojas, and W.C. Conner, Langmuir 1991, 27, 779.

22 H.D. Bale and P.W. Schmidt, Phys. Rev. Lett., 1984, 35, 956.

23 Calo and Hall (1991).

24 W.R. Grimes, in Coal Science I, M.L. Gorbaty, J.W. Larsen, and I. Wender, Eds., Academic Press, New York, 1982, p. 21.

25 O.P. Mahajan and P.L. Walker, Jr., in Analytical Methods for Coal and Coal Products, C. Kar, Jr., Ed., Vol I, Academic Press, New York, 1978, p. 125.

36 O.P. Mahajan, in Coal Structure, R.A. Meyers, Ed., Academic Press, NY, 1982, p. 51.

27 I.M. Huxham, B. Rowatt, D.C. Sherrington, and L. Tetley, L., Polymer, 1992, 33, 2768.

28 G. Kostortz, in Treatise on Material Science and Technology, Vol. 15 Neutron Scattering, G. Kostortz, Ed., Academic Press, New York, 1979, p. 227.

29 G. Porod, in Small Angle X-ray Scattering, O. Glatter, and O. Kratky, Eds., Academic Press, New York, 1982, p. 17.

30 Huxham et al. (1992).

31 P.J. Hall, W. Ruiz Machado, D. Gascon Galan, E.L. Barrientos Barria, and D.C. Sherrington, Faraday Trans., 1996, 92, 2607.

32 P.J. Hall, M. Antxustegi, and J.M. Calo, Energy and Fuels, 1998, 12, 542.

33 P.J. Hall, L.E.A.B., Berlouis, A.J. Mackinnon, J. Wilson, D. Browning, S. Dodd, S. Morris, P. Jones, and J.M. Calo, J. Alloys and Compounds, 1997, 253, 195.

34 J.S. Higgins, J.S., and H.C. Benoit, Polymers and Neutron Scattering, Oxford Science Publications, Oxford, U.K., 1994.

35 Hall et al. (1996).

36 M.D. Foster and K.F. Jensen, J. Coll. Intf. Sci., 1990, 135, 132.

37 J. Teixeira, J. Appl. Cryst. 1988, 21,781.

38 P.W. Schmidt, J. Appl. Cryst., 1991, 24, 414. 
Porod (1982).

Higgins et al. (1994).

41 Foster and Jensen (1990).

42 lbid.

43 W.H. Press, B.P. Flannery, S.A. Teukolsky, S.A., and W.T. Ketterling, Numerical Recipes - The Art of Scientific Computing, Cambridge University Press, New York, 1987.

P. Thiyagarajan, J.E. Epperson, R.K. Crawford, J.E. Carpenter, T.E. Klippert, and D.G. Wozniak, J. Appl. Cryst., 1997, 30, 1.

45 K. Vorres, Users Handbook for the Argonne Premium Coal Sample Program, ANL/PCSP-93/1, Argonne National Laboratory, Argonne, Illinois, 1993.

46 S.R. Sandler and W. and Karo, Polymer Syntheses, Academic Press, N.Y., 1977.

47 Chang and Rhee (1978)

48 M.D. Foster and K.F. Jensen, K.F., Carbon, 1991, 29, 271.

49 Porod (1982).

50 A. Benedetti, G. Fagherazzi, P. Riello, and Y.W. Zeng, J. App. Cryst., 1993, $26,717$.

51 Vorres (1993).

$52 \quad$ Foster and Jensen (1991).

53 Donnet (1982).

54 Chang and Rhee (1978).

55 Y. Yamashita and K. Ouchi (1981).

s6 M.M. Antxustegi and J.M. Calo, Proc. Carbon '97, 23rd Biennial Conf. Carbon, Penn State University, 1997, p. 404.

57 Román-Martínez et al. (1996).

58 lbid.

59 Gupta and Harrison (1994).

\section{Section 2.6: Liquid Crystal Surface Anchoring in the Formation of Carbon Structures}

Previous reports have described results of new research on the development of crystalline order in coal chars and other carbon materials. The work to date has been concerned with order in bulk phases. Ordering at surfaces, however, is also an important aspect of carbonization. The structure of carbon materials is influenced by the presence of gas interfaces, both at the external surface of the carbon body, and in internal bubble cavities. In composites, structure is influenced by ordering at the interface of the second solid phase, which may be a fiber, ceramic or metallic particle, or, in the case of coal technologies, a solid mineral grain. The arrangement of graphene layers at surfaces can influence the reactivity (by determining the ratio of edge to basal sites at the gas interfaces), and the interfacial energy and binding characteristics in composites. It also determines the proper boundary condition for mesophase pitch flow problems, such as those that describe fiber spinning.

The surface alignment of elongated molecules, either rods or disks, is a fundamental property of a liquid crystal. The "surface anchoring" is characterized by a preferred angle (the "easy axis") and a free energy associated with rotation away from that axis. Surface anchoring angles and energies have been determined experimentally for many liquid crystal / surface combinations. There has not, however, been systematic study of the surface anchoring properties of carbon material precursors. Work was undertaken this quarter to systematically determine the favored alignment of graphene layers at gas interfaces in the early stages of carbonization. The work described below was funded jointly by the current project and the NSF CAREER program in the thermal sciences, as it has direct applicability to a variety of carbon material systems and thus the goals of both programs at Brown. 
In this work a hot-stage polarizing microscope with long-focal-length optics (see Fig. 1.1) was used to obtain real-time in situ video sequences of the free surface of pools of molten pitch. The pitch was AR-3X15-1 from Mitsubishi Chemical, supplied courtesy of Professor Dan Edie at Clemson University. Mesophase pitch was chosen as a model substance rich in high-molecular-weight PAH with high fluidity, allowing characterization of the equilibrium surface anchoring properties. The mesophase pitch was contained in glass crucibles at $200-400^{\circ} \mathrm{C}$ inside a custom designed heliumpurged heated cell. Ex situ polarizing microscopy was also used to examine free surfaces and cross-sections of quenched pitch samples at the end of the hot stage experiments.

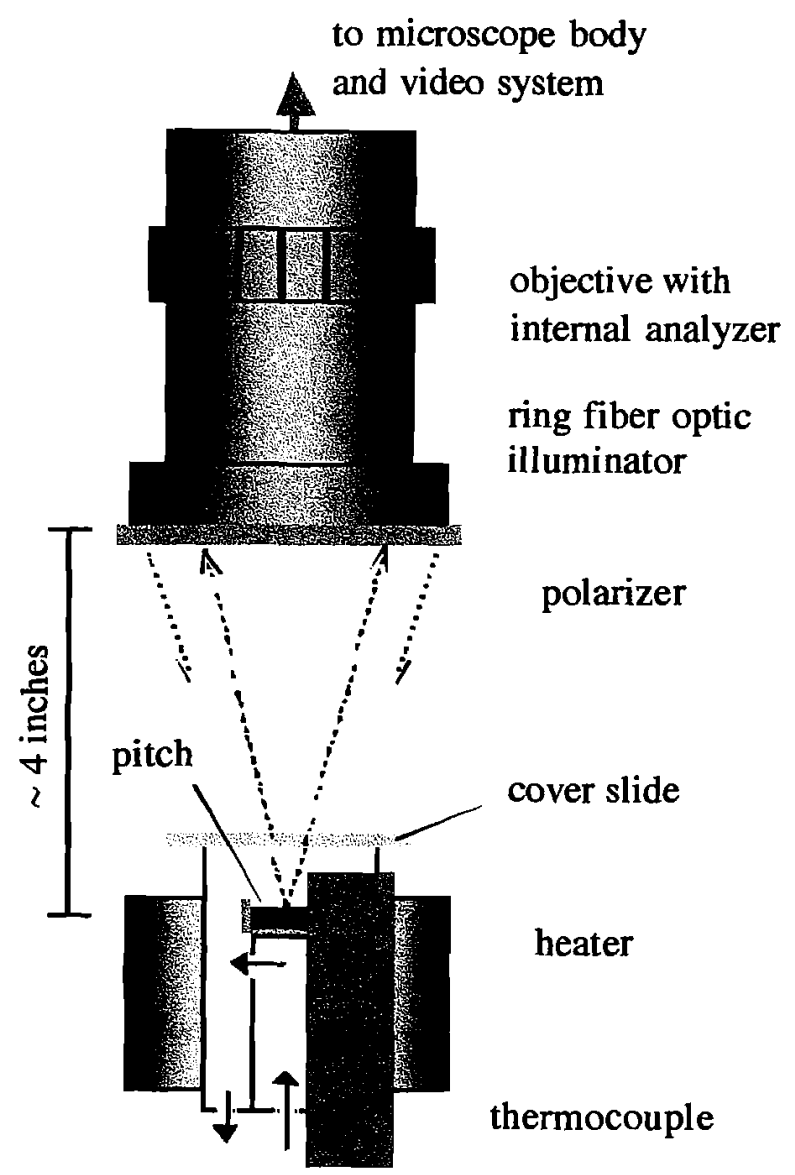

Figure 2.6-1

Sketch of the long-focal-length microscope used for in situ hot-stage video imaging of molten mesophase pitch. The long focal length allows observation of a free surface (pitch / He interface) not in contact with a glass cover slide.

illuminator

gas flow

\section{Results}

Images of the free surface of the pitch pools (not shown) show classical liquid crystal texture with well-defined flow domains and sharp disclination lines. The strong optical anisotropy of this surface suggests a significant edge-on component of the surface layer orientations. (Note that strict face-on alignment would produce an isotropic surface.) 
Strong evidence for near perpendicular edge-on orientation is seen in optical micrographs of polished cross-sections (Figs. 2.6-2 and 2.6-3 ). Figure 2.6-2 shows the gas/pitch interface at the free (top) surface in a polished cross-sections of pools of pitch, while Fig. 2.6-3 shows the gas I pitch interface at the interior surface of a bubble cavity with the pitch pool. Figure 2.6-2 shows various flow domains beneath the free surface of a pitch pool. When the flow domains consist of layers perpendicular to the free surface, the domain color extends fully to the interface. When, however, the flow domains consist of layers parallel to the surface, the domain switches to a surface film

Figure 2.6-2 Black-and-white reproduction of a color, polarized optical micrograph of a polished cross-section of quenched mesophase pitch. In the original, the dark regions are blue and the light regions are yellow. Flow domains with perpendicular alignment (white layer pairs on dark field) extend fully to the free surface. In contrast, flow domains with parallel alignment (dark layer pairs on light field) stop short of the free surface, giving way to edge-on surface films. The optical system uses crossed polars with retarder plate. Layer orientations shown were deduced from the color original following calibrating of the

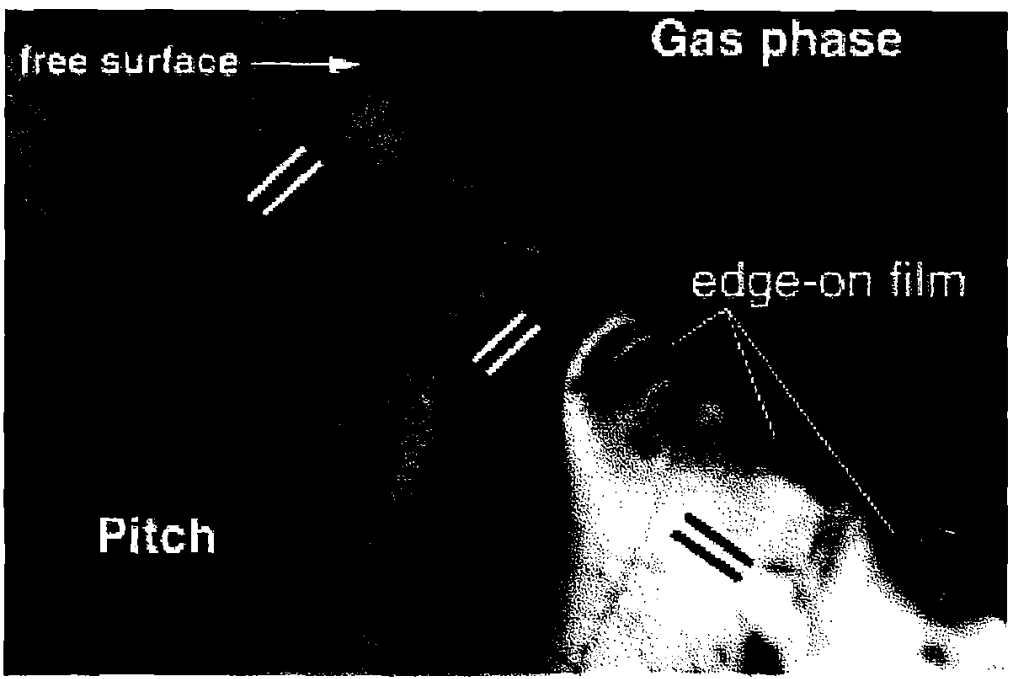
optical system using the polished edges of Ticonderoga graphite flakes.

In Figure 2.6-3, layer alignment near cavities is seen to be parallel (face-on), except for edge-on alignment in a thin film of micrometer dimensions right at the cavity surface. It is believed that the stretching and drainage of the membranes surrounding the bubbles causes parallel alignment by a flow mechanism, while the true surface equilibrium state is the opposite (edge-on) and seen only in thin surface films.

\section{Discussion}

Overall the optical microscopy clearly demonstrates preference for near-perpendicular orientation of the polyaromatic mesogens at free surfaces (layers edge-on at gas/pitch interfaces). This trend has been pointed out previously by White et al. [1982] for the particular case of mesophase spheres that intersect the free surface in two-phase pitches, but is contrary to the orientation of graphene layers 
in many carbon materials, as will be discussed later. Our interpretation

of this finding is as follows.

We have postulated that the equilibrium nanostructure in a mesophase-containing pitch is given by minimization of:

$$
G=\sum\left(n_{i} g_{i}^{0}\right)+G_{\text {mix }}+G_{\text {orient }}+G_{\text {surface }}+G_{\text {elastic }}
$$

in the absence of flows and external fields [Hu and Hurt, 2000]. In this expansion, $\mathrm{g}_{\mathrm{i}}{ }^{\mathrm{O}}$ represent the standard free energies of formation of the polycyclic aromatic building blocks as single-

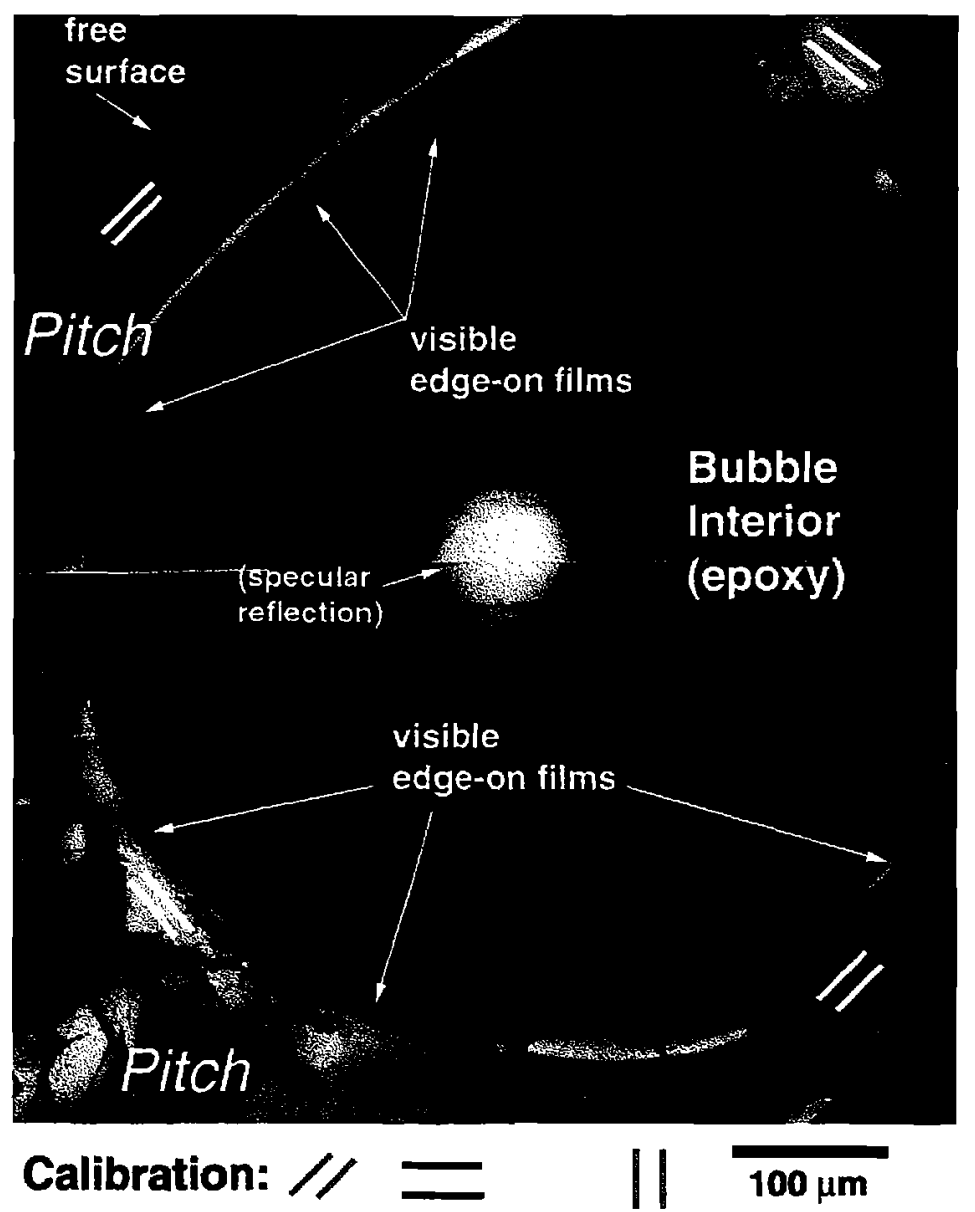

Figure 2.6-3 Optical micrograph of cross-section of quenched mesophase pitch, showing the free surface (pitch / He interface) and regions around an internal bubble. Layer orientations shown in the key were obtained by calibration using polished edges of Ticonderoga graphite flakes. 
component isotropic condensed phases; $\mathrm{n}_{\mathrm{i}}$ represent the mole numbers; and the remaining terms are contributions to the system free energy derived from mixing, orientational order, surface or interfacial energies (including orientational effects at the surface), and elastic strain due to bend or splay in the ordered phase.

This report deals with the interfacial term, $G_{\text {interface, }}$ which for gas / pitch interfaces is equivalent to the surface tension, $\gamma$. The overall interfacial tension consists of isotropic and anisotropic components:

$$
\mathrm{G}_{\text {interface }}=\gamma=\gamma_{o}+\mathrm{W}(\theta)
$$

where $\theta$ is the angle of departure of the directer from the easy axis and $W(\theta)$ is a positive function. For discotic, the director is a unit vector perpendicular to the aromatic planes. The preferred surface orientation, $\theta$, is that which minimizes $\mathrm{G}$ in $\mathrm{Eq} .2$ (and thus in equation 1.) By this principle, the microscopic observations imply that the surface tension is lower for an edge-on surface than for a surface consisting of faces, or basal planes; i.e. $\gamma_{\text {edge }}<\gamma_{\text {basal, }}$ or that the easy axis places the director perpendicular to the unit vector defining the surface. Note that crystalline graphite samples show the opposite behavior, with surface energies higher at edge faces than basal faces. These energies reflect unsaturated covalent bonds at edge sites, however, and are not representative of hydrogen-saturated polyaromatic clusters. These clusters interact by physical forces, and theoretical and experimental evidence is strongly in favor of face-to-face association as the dominant interaction (see below).

A number of other observations in the literature provide indirect support for the finding that $\gamma_{\text {edge }}<$

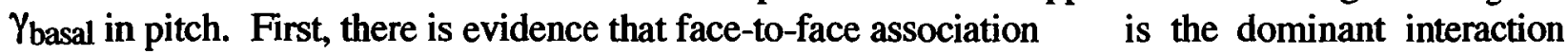
between polyaromatic clusters in the early stages of carbonization. The first structures to form in the early stages of carbonization are face-to-face associated clusters of 2-4 disk-like polyaromatic clusters, or basic structural units [Oberlin, 1965]. Also, mesophase has been observed by HRTEM to possess a partial columnar nature, again suggesting strong face-to-face association. Molecular modeling calculations by Vorpagel and Lavin [1992] on the fully-condensed PAH coronene, circumcoronene, yield binary interactions that are much stronger in the face-to-face or shifted-stack configuration than in the edge-to-edge configuration. Each of these results likely reflects the high relative strength of $\pi-\pi$ interactions. Edge-on alignment at the gas interface maximizes the internal $\pi-\pi$ interactions (it internally satisfies all faces) and thus leads to the lowest surface free energy. Secondly, aromatic compounds tend to have higher surface tensions than aliphatic compounds, and thus the aromatic basal-rich surfaces may be expected to have higher tensions than edge-rich surfaces, which are partially aliphatic due to short aliphatic side chains at the periphery of polyaromatic mesogens.

Finally, a word should be said about graphene layer orientations at interfaces in fully carbonized samples. High-temperature heat treatment causes extensive dehyrogenation and aromatization reactions that lead to edge-to-edge coalescence of the layers. The thermodynamic driving force for these chemical reactions far exceeds the thermodynamic driving forces for the alignment under the physical forces that dominate in fluid pitch [Vorpagel and Lavin,1992]. Thus graphite edge energies are higher than basal energies, due to unsaturated bonds at edge sites. Under conditions of high chemical reactivity, therefore, the equilibrium structures are those that allow satisfaction of 
reactive edge sites, which in the presence of most surfaces (such as a gas interface) is the face-on configuration. Thus there is both a physical equilibrium state for hydrogen-rich pitches in which layers are edge-on at gas interfaces, and an opposing chemical equilibrium state in hydrogendeficient carbon materials in which layers are face-on at gas interfaces. More work is needed to determine under what conditions the equilibrium edge-on films in pitch remain in a frozen metastable state during complete carbonization, and under what conditions they are capable of rearranging to the lower free energy face-on configuration during dehydrogenation and complete carbonization. Further experiments are also underway to systematically characterize the equilibrium anchoring at various solid/pitch (vs. gas/pitch) interfaces.

\section{References for Section 2.6}

White, J.L., Buechler, M., Ng, C.B., Carbon, 1982, 20, 536.

Oberlin, A., in Chemistry and Physics of Carbon, Volume 22, Marcel Dekker, Inc. New York, 1965.

Vorpagel, E.R, and Lavin, J.G., Carbon , 1992, 30, 1033.

Hurt, R.H., Crawford, G.P., Shim, H.-S., "Equilibrium Structure of Primary Soot Particles," accepted for publication in the Proceedings of the Twenty-Eighth Symposium (International) on Combustion, The Combustion Institute, Pittsburgh, 2000.

Hu, Y., Hurt, R.H., "Thermodynamics of Carbonaceous Mesophase II: General Theory for NonIdeal Solutions," submitted to Carbon, January, 2000. 


\section{Chapter 3: Computational Chemistry of Aromatic Oxidation Pathways}

\subsection{C-H and N-H Bond Dissociation Energies of Small Aromatic Hydrocarbons}

\subsubsection{Executive Summary}

A survey of computational methods was undertaken to calculate the homolytic bond dissociation energies (BDEs) of the $\mathrm{C}-\mathrm{H}$ and $\mathrm{N}-\mathrm{H}$ bonds in monocyclic aromatic molecules that are representative of the functionalities present in coal. These include 6-membered rings (benzene, pyridine, pyridazine, pyrimidine, and pyrazine) and 5-membered rings (furan, thiophene, pyrrole, and oxazole). By comparison of the calculated $\mathrm{C}-\mathrm{H}$ BDEs with the available experimental values for these aromatic molecules, the B3LYP/6-31G(d) level of theory was selected to calculate the BDEs of polycyclic aromatic hydrocarbons (PAHs), including carbonaceous PAHs (naphthalene, anthracene, pyrene, and coronene) and heteroatomic PAHs (benzofuran, benzothiophene, indole, benzoxazole, quinoline, isoquinoline, dibenzofuran, and carbazole). The cleavage of a $\mathrm{C}-\mathrm{H}$ or a $\mathrm{N}-\mathrm{H}$ bond generates a $\sigma$ radical that is, in general, localized at the site from which the hydrogen atom was removed. However, delocalization of the unpaired electron results in $\sim 7 \mathrm{kcal}^{-\mathrm{mol}^{-1}}$ stabilization of the radical with respect to the formation of phenyl when the $\mathrm{C}-\mathrm{H}$ bond is adjacent to a nitrogen atom in the azabenzenes. Radicals from 5-membered rings are $\sim 6 \mathrm{kcal}^{-\mathrm{mol}^{-1}}$ less stable than those formed from 6 -membered rings due to both localization of the spin density and geometric factors. The location of the heteroatoms in the aromatic ring affects the $\mathrm{C}-\mathrm{H}$ bond strengths more significantly than does the size of the aromatic network. Therefore, in general, the monocyclic aromatic molecules can be used to predict the C-H BDE of the large PAHs within $1 \mathrm{kcal}-\mathrm{mol}^{-1}$.

\subsubsection{Introduction}

Coal is one of the dominant fossil fuels of the twentieth century and the fuel used for most of the electrical power generation in the United States, yet much remains to be understood about its structure and reactivity. In addition, it is difficult to characterize coal because many 
properties of coal, such as elemental composition, reactivity, and rank, vary greatly depending on the origin of the sample. Although coal is a mixture of complex compounds, it has many periodic units, including heterocyclic and polycyclic aromatic components. The heteroatoms, which include nitrogen, oxygen, and sulfur, are known to play a very important role in coal combustion and processing. ${ }^{2}$ These atoms appear in coal in such forms as furan, thiophene, pyrrole, benzene, and pyridine rings.

The most important processes of coal are combustion, gasification, pyrolysis, and liquefaction. ${ }^{2}$ Each of these methods occurs under different conditions to yield different products; however, the initial reactions of combustion are thought to be common to all processes. Thus, an understanding of the thermochemistry and reactivity of specific bonds within the coal structure may lead to advances in coal processing. To this end, we are currently examining the thermochemical preferences of the aromatic constituents of coal. We hope that small molecules may be used as effective models of the components of coal in order to study the very complicated chemistry of coal combustion. We are particularly interested in identifying a computational method that is practical for the calculation of the chemical properties of computationally larger molecules, such as large polycyclic aromatic hydrocarbons (PAHs), which can be used to approximate the network nature of coal.

The first step in the combustion of coal is likely to be the homolytic cleavage of a carbonhydrogen bond to form aromatic radicals. ${ }^{3}$

$$
\mathrm{R}-\mathrm{H} \rightarrow \mathrm{R}^{*}+\mathrm{H}^{*}
$$

The bond dissociation energy (BDE) is defined as the enthalpy of the reaction required to break the $\mathrm{C}-\mathrm{H}$ bond to form two radicals. Experimental measurements of the $\mathrm{C}-\mathrm{H}$ BDEs of five- and six-membered ring compounds that are relevant to coal combustion are not abundant in the literature. Even the energy required to remove a hydrogen atom from benzene to form the phenyl radical - the first step in perhaps the most widely studied model of the fossil fuel combustion process - was not definitively determined until only three years ago. ${ }^{4}$ In that work, a combination of gas-phase ion chemistry and negative ion photoelectron spectroscopy was used to 
determine the $\mathrm{C}-\mathrm{H}$ bond strength of benzene to be $113.5 \pm 0.5 \mathrm{kcal}^{-\mathrm{mol}^{-1}}$ at $300 \mathrm{~K}$.

In addition to benzene, nitrogen-containing aromatic compounds are of interest in the modeling of coal combustion processes because nitrogen-containing constituents are known to be very important in the formation of $\mathrm{NO}_{x}$ species. ${ }^{5,6}$ Very recently, the pyrolyses of pyridine, pyrimidine, and pyrazine were experimentally explored via shock tube studies. ${ }^{7-9}$ The C-H BDEs in these azabenzenes were found to be smaller than that of benzene when the hydrogen atom was abstracted from a carbon atom adjacent to a nitrogen atom, but approximately the same as benzene when the hydrogen was removed from a carbon two or three positions away from nitrogen. The stabilization of the radical was attributed to the interaction of the unpaired electron on carbon with the adjacent nitrogen lone pair. $A b$ initio calculations at the MP2/DZP//HF/3$21 \mathrm{G}$ level supported the proposed thermochemistry of these azabenzenes; although, a difference between the calculated and experimental preferences for the site of abstraction from pyrimidine was noted. ${ }^{10}$

There are even less experimental data available for the C-H BDEs of five-membered aromatic rings. Photodissociation of pyrrole at both 193 and $248 \mathrm{~nm}$ have recently provided BDEs for the $\mathrm{N}-\mathrm{H}\left(88 \pm 2 \mathrm{kcal}^{-\mathrm{mol}^{-1}}\right)$ and $\mathrm{C}-\mathrm{H}\left(112.5 \pm 1 \mathrm{kcal}^{\left.-\mathrm{mol}^{-1}\right)}\right.$ bonds at $0 \mathrm{~K}$ via photofragment translational spectroscopy. ${ }^{11}$

Polycyclic aromatic hydrocarbons (PAH) provide a larger model with which to approximate the network nature of coal. Coals of different rank have different degrees of PAH ordering, with anthracites containing the largest aromatic fraction. ${ }^{1}$ In addition, PAHs are significant products of the combustion process, especially under $\mathrm{NO}_{\mathrm{x}}$ staging conditions. ${ }^{6}$ The bulk of the experimental studies of the physical properties of PAHs has been to identify their vibrational frequencies. ${ }^{12,13}$ It is much more difficult to determine experimentally the C-H BDE of these large species compared to the monocyclic compounds, and thus experimental C-H BDEs of the PAHs are very scarce in the literature.

An early paper that measured the $\mathrm{C}-\mathrm{H}$ bond strengths of benzene, naphthalene, and anthracene via competition experiments based on relative hydrogen atom abstraction from 
toluene concluded that the relative $\mathrm{C}-\mathrm{H}$ bond strength of PAHs could be understood as a consequence of the bonding of the two neighboring carbon atoms and not the size of the aromatic network. ${ }^{14}$ Very recently, the gas-phase acidity $\left(\Delta \mathrm{H}_{\text {acid }}\right)$ of naphthalene ${ }^{15}$ and the electron affinity (EA) of the naphthyl radicals were determined by Kass and co-workers so as to determine the C$\mathrm{H}$ bond strength of naphthalene. ${ }^{16}$ The result of those experiments indicates that the energy required to cleave each of the $\mathrm{C}-\mathrm{H}$ bond of naphthalene is $111 \pm 3 \mathrm{kcal}^{-\mathrm{mol}^{-1}}$. Insight into the C$\mathrm{H}$ bond strength of heteroatomic PAHs can also be gleaned from thermal decomposition studies of nitrogen-containing polycyclic aromatic molecules. ${ }^{17,18}$ These studies show that the decomposition reactions of the monocyclic rings are predictive of the larger ring systems.

Initially, computational calculations of $\mathrm{C}-\mathrm{H}$ BDEs of PAHs were limited to semiempirical methods due to the cost incurred in calculating the aryl radicals by more accurate methods. ${ }^{19}$ The most inexpensive ab initio method, Hartree-Fock (HF), does not include electron correlation and therefore does not accurately predict thermochemical properties such as BDEs. $^{20}$ However, computational studies of $\mathrm{C}-\mathrm{H}$ BDEs of aromatic compounds have recently received renewed attention following the more widespread use of pure and hybrid density functional theory (DFT) methods. ${ }^{21,22}$ Recent B3LYP ${ }^{23}$ and BLYP ${ }^{24,25}$ studies of PAHs containing one to six benzenoid rings have shown that DFT correctly describes the electronic structure of PAH radicals.

The aim of this work is to use computational methods to study small aromatic compounds that represent functional groups known to be present in coal. To this end, the C-H BDEs of heteroatomic 5-membered, 6-membered, and polycyclic aromatic compounds have been calculated via the hybrid DFT B3LYP method. A survey of computational methods was undertaken in the calculation of $\mathrm{C}-\mathrm{H}$ BDEs of the monocyclic aromatic compounds. On the basis of its excellent agreement with the available data from several experiments, the B3LYP ${ }^{26-29}$ level was chosen for the study of the polycyclic aromatic compounds. In a future paper we will present calculations of transition states for abstraction and addition processes to several of these model compounds via $\mathrm{H}, \mathrm{O}\left({ }^{3} \mathrm{P}\right)$, and $\mathrm{OH}$. Others have considered possible decomposition of the 
radicals in combustion environments. ${ }^{30-32}$

\subsubsection{Computational Details}

All geometry optimizations and frequency calculations were performed using Gaussian $94^{33}$ running on either an SGI Power Challenge or a Cray T3E at the Ohio Supercomputer Center. The geometries of the aromatic rings and their hydrogen atom loss products were optimized with the standard 6-31G(d) basis $\operatorname{set}^{20}$ using several computational methods, including HF, MP2, and DFT. Vibrational frequencies have also been calculated for each stationary point to verify a minimum energy structure and to provide zero-point energies (ZPEs), which were scaled by the factors of $0.9135,{ }^{34} 0.9646,{ }^{34} 0.9806,{ }^{35}$ and $1.0126^{35}$ for HF, MP2, B3LYP, and BLYP, respectively. The BPW91 zero point energies were not scaled. The frequency calculations also provided the thermal contributions to the enthalpy, which were obtained using unscaled frequencies.

In addition, BDEs were calculated using model chemistries that have been shown to reproduce quite accurately a large number of experimentally determined thermochemical properties for a test set of molecules. ${ }^{36}$ A comparison of the BDEs of a test set including more than 100 small molecules showed that each of these compound methods provided BDEs that were within $1.5 \mathrm{kcal}-\mathrm{mol}^{-1}$ of the experimental values. ${ }^{36}$ Complete Basis Set (CBS) methods are able to achieve high accuracies at reduced computational expense by extrapolation to the complete basis set limit and by inclusion of a high level of electron correlation. ${ }^{37}$ The modified Gaussian-2 method, G2MP2, is a similar composite theory that is based on the additivity of several correction terms that use larger basis sets at lower levels of theory and smaller basis sets at higher levels of theory. ${ }^{38}$

Single-point energy calculations were evaluated with the $6-311+G(d, p)$ and 6$311+\mathrm{G}(3 \mathrm{df}, 2 \mathrm{p})$ basis sets while using the B3LYP/6-31G(d) geometries. The ZPEs and thermal contributions were taken from the B3LYP/6-31G(d) frequency calculations. To analyze the trends in $\mathrm{BDE}$ as a function of the atoms in the aromatic rings, the electronic wave functions 
were calculated at the B3LYP/6-311+G(d,p)//B3LYP/6-31G(d) level. Six Cartesian d functions were used with all basis sets. Spin density populations were obtained from the wave functions via Bader's Theory of Atoms in Molecules. ${ }^{39-42}$ Spin density populations were calculated using AIMALL. ${ }^{43}$ The spin density is the difference in the $\alpha$ and $\beta$ spin densities. Its magnitude is a measure of the extent of delocalization of the radical character throughout the molecule. ${ }^{44}$

The chemistry under consideration is the energy required to cleave the $\mathrm{C}-\mathrm{H}$ bond in small aromatic hydrocarbons. Because these compounds will be used as models for studying the combustion process, it is relevant to ascertain the effects of increased temperatures on the C-H bond strength. The enthalpies of the monocyclic aromatic rings and the radicals resulting from cleavage of each unique $\mathrm{C}-\mathrm{H}$ bond were calculated from $300 \mathrm{~K}$ to $2000 \mathrm{~K}$ by including a temperature-dependent enthalpic term, ${ }^{20}$

$$
\Delta \mathrm{H}(\mathrm{T})=\mathrm{H}_{\text {trans }}(\mathrm{T})+\mathrm{H}_{\mathrm{rot}}(\mathrm{T})+\mathrm{H}_{\mathrm{vib}}(\mathrm{T})+\mathrm{RT}
$$

where the summation is over all $3 \mathrm{~N}-6$ normal modes. The scaled zero point energy and the enthalpy contribution, computed by the above equation, were added to the B3LYP total energy to determine the $\mathrm{C}-\mathrm{H}$ BDEs as a function of temperature.

\subsubsection{Results}

The BDEs of a number of monocyclic aromatic compounds, calculated by several different methodologies, are presented in Table 3.1.1 (for atom numbering, see Figures 3.1.1 and 3.1.2). Where available, the calculated BDEs were compared to the experimental values in order to obtain an "average error" for each method (see the bottom row of Table 3.1.1). The results are in agreement with the findings of others ${ }^{24,45}$ that HF and MP2 methods cannot be used to adequately describe both the parent aromatic molecule and the radical resulting from hydrogen atom loss. The lack of electron correlation at the HF level leads to an underestimation of the C-

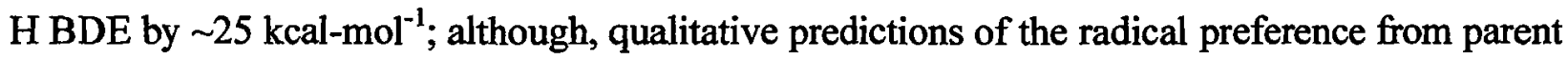
aromatics with more than one unique $\mathrm{C}-\mathrm{H}$ bond were reliable. In addition, both HF and MP2 calculations of the radical species suffer from significant spin contamination, with $\left(\mathrm{S}^{2}\right)$ as high as 
1.4 for the radicals formed from benzene and the azabenzenes. As a result, most of the MP2 BDEs calculated were $\sim 17.0 \mathrm{kcal}^{-\mathrm{mol}^{-1}}$ higher than experiment. Furthermore, a comparison of

Table 3.1.1. The calculated C-H (and N-H) BDEs of the monocyclic aromatic molecules at $298 \mathrm{~K}$ (in kcal-mol ${ }^{-1}$ )

\begin{tabular}{|c|c|c|c|c|c|c|c|c|c|c|}
\hline Parent & $\operatorname{Site}^{a}$ & State & CBS-4 & $\overline{\text { CBS-q }}$ & CBS-Q & G2MP2 & BLYP & BPW91 & $\overline{B 3 L Y P}$ & Expt \\
\hline benzene & 1 & ${ }^{2} \mathrm{~A}_{1}$ & 117.2 & 119.1 & 114.7 & 117.2 & 108.6 & 107.3 & 110.8 & $\overline{113.5 \pm 0.5^{b}}$ \\
\hline \multirow[t]{3}{*}{ pyridine } & 2 & ${ }^{2} A^{\prime}$ & 112.0 & 112.0 & 110.3 & 113.1 & 102.8 & 101.9 & 105.6 & $105 \pm 2^{c}$ \\
\hline & 3 & ${ }^{2} A^{\prime}$ & 119.0 & 120.5 & 116.6 & 118.5 & 107.9 & 107.0 & 111.3 & $112 \pm 2^{c}$ \\
\hline & 4 & ${ }^{2} \mathrm{~A}$, & 116.8 & 118.2 & 115.3 & 117.5 & 107.1 & 106.0 & 110.0 & $112 \pm 2^{c}$ \\
\hline \multirow[t]{2}{*}{ pyridazine } & 3 & ${ }^{2} A^{\prime}$ & 117.2 & 118.0 & 114.1 & 116.8 & 103.8 & 103.1 & 107.0 & \\
\hline & 4 & ${ }^{2} A^{\prime}$ & 120.8 & 122.1 & 115.5 & 118.3 & 103.8 & 103.0 & 108.9 & \\
\hline \multirow[t]{3}{*}{ pyrimidine } & 2 & ${ }^{2} \mathrm{~A}_{1}$ & 112.5 & 112.7 & $f$ & 114.8 & 104.6 & 103.5 & 107.2 & $98 \pm 2^{c}$ \\
\hline & 4 & ${ }^{2} A^{\prime}$ & 110.9 & 110.3 & 110.6 & 112.7 & 101.4 & 100.6 & 104.7 & $103 \pm 2^{c}$ \\
\hline & 5 & ${ }^{2} A^{\prime}$ & 118.7 & 120.0 & 117.7 & 119.8 & 108.5 & 107.6 & 112.2 & $112 \pm 2^{c}$ \\
\hline pyrazine & 1 & ${ }^{2} A^{\prime}$ & 115.3 & 114.9 & 111.7 & 114.6 & 101.9 & 101.2 & 105.7 & $103 \pm 2^{c}$ \\
\hline \multirow[t]{2}{*}{ furan } & 2 & ${ }^{2} \mathrm{~A}$ & 120.1 & 120.6 & 121.0 & 123.1 & 116.2 & 115.0 & 118.4 & \\
\hline & 3 & ${ }^{2} A^{\prime}$ & 120.5 & 120.5 & 121.4 & 123.2 & 116.0 & 114.9 & 118.3 & \\
\hline \multirow[t]{2}{*}{ thiophene } & 2 & ${ }^{2} \mathrm{~A}$ & 118.8 & 119.1 & 119.2 & 121.5 & 114.4 & 113.5 & 116.6 & \\
\hline & 3 & ${ }^{2} \mathrm{~A}$ & 116.6 & 116.3 & 117.0 & 119.4 & 111.4 & 110.4 & 113.8 & \\
\hline \multirow[t]{3}{*}{ pyrrole } & 2 & ${ }^{2} \mathrm{~A}$ & 119.8 & 119.9 & 120.8 & 122.4 & $115.8^{g}$ & 114.7 & 118.1 & $112.3 \pm 1^{d}$ \\
\hline & 3 & ${ }^{2} A^{\prime}$ & 119.6 & 119.5 & 120.4 & 121.9 & 115.6 & 114.3 & 117.6 & $112.3 \pm 1^{d}$ \\
\hline & $\mathrm{n}$ & ${ }^{2} \mathrm{~A}_{2}$ & 96.2 & 94.4 & $h$ & $h$ & 87.5 & 88.3 & 89.8 & $\begin{array}{c}88 \pm 2^{d} \\
96.6 \pm 4^{e}\end{array}$ \\
\hline avg. error ${ }^{i}$ & & & 7.9 & $\overline{8.4}$ & 5.3 & 8.9 & 3.6 & 3.9 & 3.1 & \\
\hline
\end{tabular}

${ }^{a}$ The radical site (see Figures 3.1.1 and 3.1.2). ${ }^{b}$ Ref. 4. ${ }^{c}$ Ref. 9. ${ }^{d}$ Ref. 11. ${ }^{e}$ Ref. 54. ${ }^{f}$ Error converging wavefunction. ${ }^{g} C_{l}$ symmetry. ${ }^{h}$ The complicated electronic structure of pyrrole prohibited the calculation of the N-H BDE at the MP2, CBS-Q, and G2MP2 levels. The HF, CBS-4, and CBS-q calculations are based on a $C_{l}$ symmetry. ${ }^{i}$ Average of the absolute error of (experiment - theory) for $\mathrm{C}-\mathrm{H}$ bonds. 
the model chemistries and the experimental data available for the small aromatic rings reveals that the CBS and G2MP2 methods fall short of the accuracy predicted by the test set of molecules. While there is excellent agreement with the experimental C-H BDE of benzene by CBS-Q, the G2MP2 level predicts a higher BDE than the accurate experimental value. For comparison to the other experimental values, the average error for the CBS and G2MP2 methods is slightly beyond their typical $2 \mathrm{kcal}^{-\mathrm{mol}^{-1}}$ error. It should also be noted that all of the CBS methods perform better than the G2MP2 method. However, it has been noted previously that G2 methods fail to accurately predict the properties of aromatic hydrocarbons. ${ }^{46}$ It has recently been recognized that CBS- $R A D$, a modification of the CBS-Q method, is more suited to the accurate calculation of the thermochemical properties of free radicals. ${ }^{47}$ However, the use of QCISD for both the optimization and zero point determination steps within the CBS-RAD method render the CBS-RAD method impractical for the size of the molecules considered in this study.

The C-H bond enthalpies calculated via each of the DFT methods are in better agreement with experiment than the methods based on HF theory. The pure DFT methods, such as BLYP and BPW91, yielded BDEs that are on average lower than experiment by $\sim 3-4 \mathrm{kcal}^{-\mathrm{mol}^{-1}}$. The

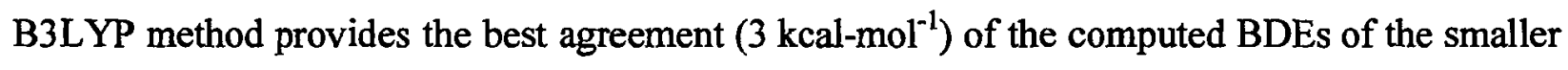
aromatic hydrocarbons with experiment. It should be noted that the qualitative trends for the BDEs are reproduced by all of the CBS, G2MP2 and the DFT methods. However, the DFT methods are more affordable than the CBS or G2-based methods and are seemingly well-suited for the study of large PAHs. For these reasons, B3LYP was selected to elucidate the trends in the calculated bond dissociation enthalpies of the monocyclic aromatic molecules as well as to study larger carbonaceous and heteroatomic PAHs.

To determine the validity of the $6-31 \mathrm{G}(\mathrm{d})$ basis set, B3LYP single point calculations were performed using the $6-311+G(d, p)$ and $6-311+G(3 d f, 2 p)$ basis sets. Bond dissociation enthalpies were determined by adding scaled zero point and thermal contributions from the 6-31G(d) basis set calculation to the energies calculated with the larger basis set for both the parent and carbon- 
centered radical. It is evident from a comparison of the B3LYP C-H BDEs with the three different basis sets (Table 3.1.2) that a larger basis set leads to negligible differences in comparison to experiment in the predicted C-H BDEs. Most importantly, the relative stability of the radicals remains unchanged from that predicted via the smaller 6-31G(d) basis set upon the inclusion of basis functions of higher angular momentum and diffuse functions. Not surprisingly, the bond strength of the more polar $\mathrm{N}-\mathrm{H}$ bond is affected by the size of the basis set. Inclusion of higher angular momentum basis functions results in a slightly higher N-H BDE in pyrrole by $\sim 4 \mathrm{kcal}^{-\mathrm{mol}^{-1}}$.

\subsubsection{C-H BDE of Benzene}

The B3LYP/6-31G(d) calculations for the dissociation of a hydrogen atom from benzene to form the phenyl radical will serve as the standard for comparison with the other aromatic hydrocarbons presented in this work. To accurately predict the $\mathrm{C}-\mathrm{H}$ bond strength the computational method must first reproduce the geometry of both the parent and the radical. The $\mathrm{C}-\mathrm{C}$ and $\mathrm{C}-\mathrm{H}$ bond lengths for benzene calculated at the B3LYP level are in excellent agreement ( $\Delta=-0.0005 \AA$ and $\Delta=+0.003 \AA$, respectively) with those determined by high resolution spectroscopy. ${ }^{48}$ Hydrogen abstraction from benzene gives rise to a widening of the $\mathrm{C}-\mathrm{C}-\mathrm{C}$ bond angle at the radical site by $5.7^{\circ}$ and a shortening of the adjacent $\mathrm{C}-\mathrm{C}$ bonds by $0.019 \AA$. The unpaired electron in the phenyl radical is located in an $\mathrm{sp}^{2}$ orbital that is orthogonal to the $\pi$ system. However, analysis of the spin density suggests that spin polarization occurs in both the $\sigma$ and $\pi$ systems (see discussion). The C-H BDE of benzene calculated at the B3LYP level is $110.8 \mathrm{kcal}-\mathrm{mol}^{-1}$ at $298 \mathrm{~K}$, which is very close to the most recent experimental values of $111.2 \pm$ $0.8^{49}$ and $113.5 \pm 0.5^{4} \mathrm{kcal}_{-\mathrm{mol}^{-1}}$ at $300 \mathrm{~K}$, both determined via thermochemical cycles from EA and $\Delta \mathrm{H}_{\text {acid }}$ measurements. 
Table 3.1.2. A Comparison of Basis Sets for the B3LYP Calculation of BDEs at 298K (in kcal-mol ${ }^{-1}$ )

\begin{tabular}{|c|c|c|c|c|c|}
\hline Parent & Site $^{a}$ & $6-31 G(d)$ & $\overline{6-311+G(d, p)}$ & $6-311+G(3 d f, 2 p)$ & Experiment \\
\hline benzene & 1 & 110.8 & 110.6 & 110.6 & $113.5 \pm 0.5^{b}$ \\
\hline \multirow[t]{3}{*}{ pyridine } & 2 & 105.6 & 105.0 & 104.9 & $105 \pm 2^{c}$ \\
\hline & 3 & 111.3 & 111.1 & 111.1 & $112 \pm 2^{c}$ \\
\hline & 4 & 110.0 & 109.7 & 109.8 & $112 \pm 2^{c}$ \\
\hline \multirow[t]{2}{*}{ pyridazine } & 3 & 107.0 & 106.5 & 106.6 & \\
\hline & 4 & 108.9 & 108.6 & 108.7 & \\
\hline \multirow[t]{3}{*}{ pyrimidine } & 2 & 107.2 & 106.8 & 106.6 & $98 \pm 2^{c}$ \\
\hline & 4 & 104.7 & 104.2 & 104.2 & $103 \pm 2^{c}$ \\
\hline & 5 & 112.2 & 112.0 & 112.1 & $112 \pm 2^{c}$ \\
\hline pyrazine & 1 & 105.7 & 105.2 & 105.0 & $103 \pm 2^{c}$ \\
\hline \multirow[t]{2}{*}{ furan } & 2 & 118.4 & 118.7 & 118.7 & \\
\hline & 3 & 118.3 & 118.4 & 118.6 & \\
\hline \multirow[t]{2}{*}{ thiophene } & 2 & 116.6 & 116.6 & 116.6 & \\
\hline & 3 & 113.8 & 113.7 & 113.8 & \\
\hline \multirow[t]{3}{*}{ pyrrole } & 2 & 118.1 & 118.3 & 118.4 & $112.3 \pm 1^{d}$ \\
\hline & 3 & 117.6 & 117.7 & 117.8 & $112.3 \pm 1^{d}$ \\
\hline & $\mathbf{n}$ & 89.8 & 94.3 & 93.7 & $88 \pm 2^{d}, 96.6 \pm 4^{e}$ \\
\hline avg. error ${ }^{e}$ & & 3.1 & $\mathbf{3 . 0}$ & $\mathbf{3 . 0}$ & \\
\hline
\end{tabular}

${ }^{a}$ The radical site. ${ }^{b}$ Ref. $4 .{ }^{c}$ Ref. 9. ${ }^{d}$ Ref. $11 .{ }^{e}$ Ref. 54. ${ }^{e}$ Average of the absolute error of (experiment - theory) for $\mathrm{C}-\mathrm{H}$ bonds.

\subsubsection{BDEs of azabenzenes: pyridine, pyridazine, pyrimidine, and pyrazine.}

Recent experimental results have added considerably to the understanding of the thermochemistry of the azabenzenes. ${ }^{7-9}$ These studies show that the pyrolyses of pyridine, pyrimidine, and pyrazine are initiated by the homolysis of the $\mathrm{C}-\mathrm{H}$ bond with a barrier that is lower than the barrier for cleavage of the $\mathrm{C}-\mathrm{H}$ bond in benzene. The lower $\mathrm{C}-\mathrm{H}$ BDEs for the $\mathrm{C}$ $\mathrm{H}$ bonds next to the nitrogen atoms in the azabenzenes was attributed to the neighboring $\mathrm{N}-\mathrm{C}$ 
interactions that lead to stabilization of the radical via resonance. ${ }^{7-9}$ An earlier theoretical study also explored the relative stabilities of the pyridyl and diazyl radicals using the MP2/DZP//RHF/3-21G level of theory. ${ }^{10}$

The work presented here further examines the relative stabilities of these radicals as well as the radicals formed from pyridazine and pyrazine using a variety of $a b$ initio methods. The energy required to remove a hydrogen atom from the azabenzenes is greatly influenced by the presence of a nitrogen atom in the 6-membered aromatic ring. For example, the B3LYP method predicts that $\mathrm{C}-\mathrm{H}$ bond cleavage from the carbon adjacent to the nitrogen (the 2 position) in

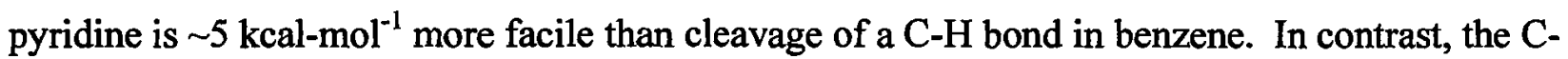
$\mathrm{H}$ bond cleavages at the 3 or 4 positions of pyridine are quite similar to that of benzene, with only a $\sim 1 \mathrm{kcal}^{-\mathrm{mol}^{-1}}$ preference for abstraction from the 4 position over the 3 position. The B3LYP calculations can be compared with a high temperature pyrolysis study of pyridine that yielded $\mathrm{C}-\mathrm{H}$ BDEs at each of the unique carbon positions in pyridine via an analysis of the decomposition products. ${ }^{9}$ The results of this shock tube study agree quite well with the B3LYP calculations in the prediction of the low $\mathrm{C}-\mathrm{H}$ BDE for the 2 position of pyridine. The experiment was unable to determine a preference for hydrogen atom loss from the 3 or 4 position of pyridine.

In general, there is good agreement between the present work and the previous calculations, as well as with the available experimental data for the C-H BDEs of 6-membered aromatic rings. However, calculations of the relative stability of the three radicals formed from pyrimidine differ from the order determined experimentally. ${ }^{8,9}$ An RRKM analysis of shock tube experiments indicates that the 2-pyrimidyl radical is the most stable radical of pyrimidine, followed by the 4-pyrimidyl and 5-pyrimidyl radicals, with C-H BDEs of 98, 103, and $112 \mathrm{kcal}-$ mol $^{-1}$, respectively. ${ }^{9}$ The corresponding B3LYP C-H BDEs are 107.2, 104.7, and $112.2 \mathrm{kcal}-$ $\mathrm{mol}^{-1}$, respectively. Resonance structures of the 2-pyrimidyl radical were invoked by Kiefer and coworkers to rationalize its stability. However, the previous theoretical study ${ }^{10}$ and the current calculations predict that the radical stability decreases in the order 4-pyrimidyl, 2-pyrimidyl, and 
5-pyrimidyl. The effect of nitrogen atoms on the strength of $\mathrm{C}-\mathrm{H}$ bonds in aromatic systems will be explored in greater detail in a forthcoming paper.

\subsubsection{BDEs of 5-membered aromatic rings: furan, thiophene, pyrrole, and oxazole.}

To date, few experiments have been performed to determine the $\mathrm{C}-\mathrm{H}$ bond strengths in 5-membered heterocyclic molecules. Unlike the azabenzenes, the thermal decomposition reactions of furan and pyrrole are not initiated by the cleavage of a $\mathrm{C}-\mathrm{H}$ bond. Instead, the experiments suggest that 5-membered heterocyclic compounds, including furan and pyrrole, isomerize or decompose via unimolecular reactions that involve scission of the $\mathrm{C}-\mathrm{O}$ or $\mathrm{C}-\mathrm{N}$ bond. ${ }^{50-52}$

The calculated BDEs of the 5-membered aromatic molecules average $\left(117.1 \mathrm{kcal}-\mathrm{mol}^{-1}\right)$, higher than that for the 6-membered rings $\left(108.3 \mathrm{kcal}^{-\mathrm{mol}^{-1}}\right)$. Also, cleavage of a C-H bond in a 5 -membered ring requires $\sim 6 \mathrm{kcal}^{-\mathrm{mol}^{-1}}$ more energy than breaking a $\mathrm{C}-\mathrm{H}$ bond in benzene. From this observation, the dissociation of a $\mathrm{C}-\mathrm{H}$ bond in 5-membered rings seemingly requires more energy because these rings are less able to accept the geometric perturbation that follows cleavage of a C-H bond. Furthermore, in contrast to the 6-membered rings, the preference for abstraction within the 5-membered rings varies only moderately between sites in the 5membered rings. In particular, the stabilization of the ortho radicals (2 position) that was observed in the 6-membered aromatic rings is absent in the 5-membered rings. For example, the preference for abstraction from the 3 position over the 2 position in furan is small $(<0.1 \mathrm{kcal}-$ $\mathrm{mol}^{-1}$ ). However, in the case of pyrrole, and even more so in the case of thiophene, hydrogen

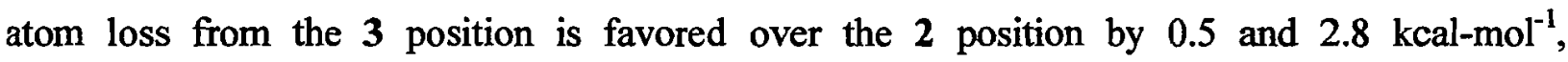
respectively. The origin of this effect will be discussed below.

Pyrrole differs from the other molecules studied in that it is an aromatic heterocyclic

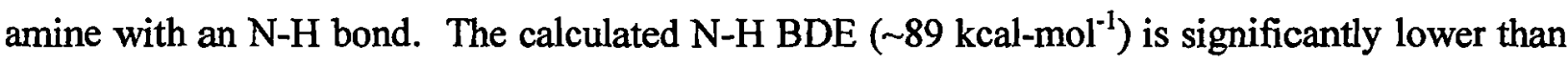
the average C-H BDE of the other aromatic molecules, but is in excellent agreement with the $0 \mathrm{~K}$ $\mathrm{N}-\mathrm{H}$ BDE determined via photofragment translational spectroscopy $\left(-88 \mathrm{kcal}-\mathrm{mol}^{-1}\right){ }^{11}$ The same 
experimental work reported that cleavage of one of the two $\mathrm{C}$ - $\mathrm{H}$ bonds occurred at $\sim 112 \mathrm{kcal}-$ $\mathrm{mol}^{-1}$, but did not distinguish the site of the bond cleavage.

The $\mathrm{C}-\mathrm{H}$ BDEs in oxazole demonstrate that simultaneous oxygen and nitrogen atom substitution provides similar perturbations in a five-membered ring, and there is little, if any, cooperativity.

\subsubsection{BDE of polycyclic and polyheteroatomic rings.}

The agreement between the experiment and calculated C-H BDEs for the monocyclic compounds provides confidence that the B3LYP method can be used to predict reliably the bond strengths of PAHs, for which few quantitative experimental values are available for comparison. BDE calculations were carried out for both polycyclic and polycyclic heteroatomic aromatic hydrocarbons.

\subsubsection{4.a. Carbonaceous PAHs}

PAHs comprised of only fused benzene rings are found to exhibit the least amount of variation in $\mathrm{C}-\mathrm{H}$ bond strengths. The $\mathrm{C}-\mathrm{H}$ BDEs for representative benzenoid PAHs of two, three, four, and seven fused benzene rings (naphthalene, anthracene, pyrene, and coronene) vary little from the predicted $\mathrm{C}-\mathrm{H}$ bond strength of benzene. Naphthalene is the only PAH in which an assessment of the $\mathrm{C}-\mathrm{H}$ bond strengths relative to benzene has been measured experimentally. The B3LYP method predicts that the energies required to cleave the $\mathrm{C}-\mathrm{H}$ bond to form the 1naphthyl radical $\left(111.1 \mathrm{kcal}^{-\mathrm{mol}^{-1}}\right)$ and the 2-naphthyl radical $\left(110.9 \mathrm{kcal}^{-\mathrm{mol}^{-1}}\right)$ differs from the energy to cleave the $\mathrm{C}-\mathrm{H}$ bond in benzene by only 0.3 and $0.1 \mathrm{kcal}-\mathrm{mol}^{-1}$, respectively. These values agree quite well with both a previous DFT calculation ${ }^{24}$ and the very recent gas-phase ion chemistry experiment that determined the $\mathrm{C}-\mathrm{H}$ bond strength to be $111 \pm 3 \mathrm{kcal}^{-\mathrm{mol}^{-1}}$ for naphthalene. ${ }^{16}$

Previous DFT computational studies of PAHs, including several PAHs larger than those in the present study, concluded that the preference for hydrogen atom loss from a particular site 
could be understood in terms of steric congestion. ${ }^{24,25}$ The PAHs chosen in this study do not include closely positioned hydrogens and thus a different explanation must be offered. An earlier MNDO study ${ }^{14}$ suggested that the energy required to cleave a $\mathrm{C}-\mathrm{H}$ bond in a PAH could be predicted by the bonding of the two neighboring carbon atoms, though the conclusions drawn here differ considerably. For example, anthracene has three unique $\mathrm{C}-\mathrm{H}$ bond positions: the 1 position is adjacent to both a secondary and a tertiary carbon; the $\mathbf{2}$ position is adjacent to two secondary carbons; and the 9 position is adjacent to two tertiary carbons. The MNDO study found that the $\mathrm{C}-\mathrm{H}$ bond at the 9 position in anthracene was several $\mathrm{kcal}^{-\mathrm{mol}^{-1}}$ weaker than that of a $\mathrm{C}-\mathrm{H}$ bond in benzene. However, the B3LYP calculations predict that hydrogen atom loss from site 2 in anthracene, the position in which the $\mathrm{C}-\mathrm{H}$ bond is most like a $\mathrm{C}-\mathrm{H}$ bond in benzene, is favored over abstraction from sites 1 and 9 that are adjacent to a bridging $C$, but only by $\leq 0.5 \mathrm{kcal}-\mathrm{mol}^{-1}$. The preferences for abstraction presented here differ slightly from the previous DFT work, ${ }^{24}$ yet preserves the preference for abstraction at the least reactive carbon site, if only by $0.1 \mathrm{kcal}^{-\mathrm{mol}^{-1}}$.

\subsubsection{4.b. Heteroatomic PAHs: 6-Membered Rings Fused to Benzene}

We have also considered polycyclic PAHs that contain heteroatoms. It is evident that the predicted C-H BDEs of the polycyclic aromatic rings are also governed by both the position of the $\mathrm{C}-\mathrm{H}$ bond with respect to the heteroatoms in the ring as well as the number of atoms in the ring. A greater variation in the strength of $\mathrm{C}-\mathrm{H}$ bonds is observed in the 6-membered bicyclic PAHs that contain nitrogen. The 2 position is the only $\mathrm{C}-\mathrm{H}$ bond adjacent to nitrogen in quinoline, whereas isoquinoline has two such sites. The 1 position in isoquinoline is adjacent to both a nitrogen and a bridging carbon whereas the 3 site is adjacent to a nitrogen and a carbon that is next to a bridging carbon. The B3LYP calculations predict that the radical formed at the 1 site is $\sim 2.5 \mathrm{kcal}^{-\mathrm{mol}^{-1}}$ more stable than that from the 3 site. This is in agreement with a recent thermal decomposition study that found that the most favored pathway for decomposition of isoquinoline was initiated by hydrogen atom loss from the 1 position. ${ }^{18}$ The experimental 
analysis of the decomposition of the quinolines was simplified by assuming that all of the $\mathrm{C}-\mathrm{H}$ bonds on the fused benzene ring possessed the same bond strength. However, the B3LYP calculations suggest that there is $\sim 2 \mathrm{kcal}^{-\mathrm{mol}^{-1}}$ variation in the strength of the four benzene-like $\mathrm{C}-\mathrm{H}$ bonds in quinoline, with the most energy being required to cleave the $\mathrm{C}-\mathrm{H}$ bond at the 8 position.

\subsubsection{4.c. Heteroatomic PAHs: 5-Membered Rings Fused to Benzene.}

Oxygen and sulfur are present in coal in several forms due to the variety of the original plant functionalities. ${ }^{2}$ However, as the process of coalification progresses, aliphatic structures are converted to heterocyclic structures and finally to heteroaromatic structures. Condensed furans and thiophenes are also present in the products of coal processing, which has been rationalized by their stability at low temperatures. ${ }^{2}$ If the initiation step in the combustion process is cleavage of a $\mathrm{C}-\mathrm{H}$ bond, then the B3LYP prediction of relatively higher $\mathrm{C}-\mathrm{H}$ BDEs for aromatic molecules that contain 5-membered rings is in agreement with these findings.

Similar to their monocyclic analogues, there is a paucity of experimental thermochemical data for the polycyclic molecules that contain 5-membered rings fused to a benzene ring. A thorough experimental study of the decomposition of indole indicated that hydrogen atom loss from indole is more facile than in pyrrole. ${ }^{17}$ However, a C-H bond strength was not reported for indole because the proposed mechanism suggests that indole first isomerizes to indolenine, followed by hydrogen atom elimination $\left(\Delta \mathrm{H}_{\mathrm{C}-\mathrm{H}}=70 \mathrm{kcal}-\mathrm{mol}^{-1}\right)$ from the $\mathrm{sp}^{3}$ carbon at the 3 position. Our B3LYP/6-31G(d) calculations suggest that this isomerization requires less than 7

$\mathrm{kcal}^{-\mathrm{mol}^{-1}}$ and that the subsequent hydrogen atom loss from the $\mathrm{sp}^{3}$ carbon at the 3 site in

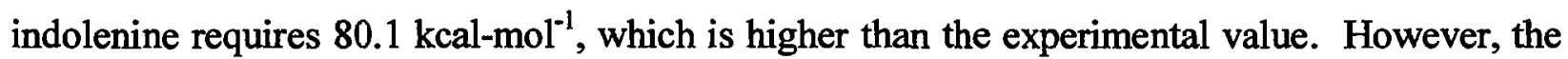
calculated N-H bond strength ( $86.9 \mathrm{kcal}^{\left.-\mathrm{mol}^{-1}\right)}$ is in good agreement with the experimental value

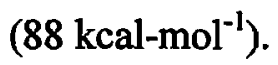




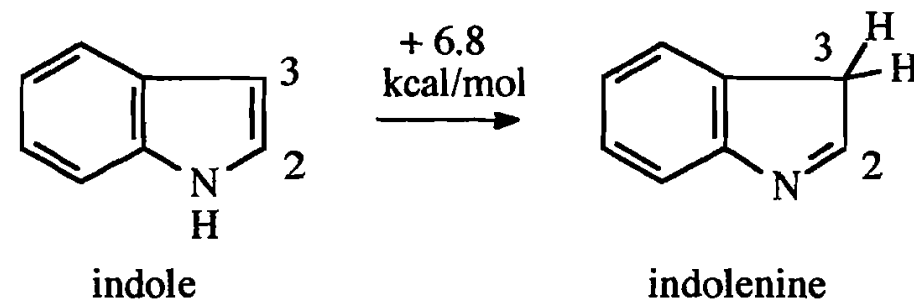

Although there is a range of $\mathrm{C}-\mathrm{H}$ BDE values for a particular site in a PAH and the corresponding site in the monocyclic aromatic compound, there is considerably more variation in the N-H BDEs. A comparison of the N-H BDE in the related compounds pyrrole, indole, and carbazole will demonstrate the effect that the aromatic network has on the N-H BDE. The calculations predict the N-H BDEs to be $89.8,86.9$, and $85.1 \mathrm{kcal}^{-\mathrm{mol}^{-1}}$ for pyrrole, indole, and carbazole, respectively. These same N-H BDEs were experimentally measured in DMSO solution and adjusted to yield gas-phase values, yielding N-H BDEs of 96.6, 93.8, and $92.7 \mathrm{kcal}-$ mol $^{-1}$ for pyrrole, indole, and carbazole, respectively. ${ }^{53}$ Although the absolute B3LYP calculated N-H BDEs differ by slightly more than the $4 \mathrm{kcal}^{-\mathrm{mol}^{-1}}$ experimental uncertainty noted by the authors, the qualitative trends are reproduced. The relative energies obtained by each method are the same. As the number of benzene rings fused to pyrrole increases, the $\mathrm{N}-\mathrm{H}$ BDE is calculated to decrease by 2.9 and $4.7 \mathrm{kcal}^{-\mathrm{mol}^{-1}}$, similar to the experimental differences of 2.8 and $3.9 \mathrm{kcal}^{-\mathrm{mol}^{-1}}$.

\subsubsection{Discussion}

\subsubsection{1.a. General Trends in Bond Dissociation Enthalpies: Geometries}

A consideration of both the geometric and electronic properties of the parent compounds and their radicals can lead to an understanding of the calculated C-H BDEs. The formation of a radical following hydrogen atom loss from a parent aromatic molecule is accompanied by regular changes in the optimized geometry. As we previously noted, when the $\mathrm{C}-\mathrm{H}$ bond in benzene is cleaved to form the phenyl radical, there is a widening of the bond angle at the site where the radical is formed and a shortening of the adjacent $\mathrm{C}-\mathrm{C}$ bonds. In addition, in order to accommodate the unpaired electron there is a reduction of the bond angles adjacent to the radical site for the aromatic ring. 
In general, the presence of a nitrogen atom in a 6-membered ring aromatic system results in a stabilization of radicals formed via $\mathrm{C}-\mathrm{H}$ bond cleavage. The hybridization of each atom in the azabenzenes is nearly of pure $\mathrm{sp}^{2}$ character and the nitrogen electron pair is in an $\mathrm{sp}^{2}$ orbital. As a result the internal bond angles of the azabenzenes are $\sim 120^{\circ}$. Upon cleavage of a $\mathrm{C}-\mathrm{H}$ bond, the bond angle at the radical site is increased by as much as $5^{\circ}$. The departure from the ideal $\mathrm{sp}^{2}$ bond angle is correlated with a reduction in the stability of the radical and an increase in the $\mathrm{C}-\mathrm{H}$ BDE of a 6-membered ring. This geometric evidence can be used to rationalize the relative stability of the radicals formed from pyrimidine as predicted by B3LYP. The bond angle widens at the radical site by $2.5^{\circ}, 3.8^{\circ}$ and $5.0^{\circ}$ following hydrogen atom loss from the 4,2 , and 5 carbon positions, respectively, which is well correlated with the B3LYP prediction that the stability decreases in the order 4-pyrimidyl, 2-pyrimidyl, and 5-pyrimidyl radicals.

Similar geometric arguments can provide insight into the calculated C-H BDEs of the 5membered aromatic molecules. A regular pentagon has internal angles of $108^{\circ}$. However, the aromatic 5-membered rings are distorted by the presence of the heteroatom $(X)$. The resulting ring possesses shortened $\mathrm{C}-\mathrm{X}$ bonds and a contracted $\mathrm{C}-\mathrm{X}-\mathrm{C}$ bond angle. The perturbation of the ring is greatest in thiophene where the C-X-C bond angle is significantly smaller $\left(\sim 91^{\circ}\right)$ than that of furan and pyrrole $\left(\sim 107-110^{\circ}\right)$.

Five-membered heteroatomic aromatic rings also have unequal contributions to the $\pi$ system from the $\mathrm{C}$ and $\mathrm{X}$ atoms. Each of the heteroatoms of the 5-membered rings must donate two electrons to the $\pi$ system to become aromatic. The hybridization of carbon in benzene is $\mathrm{sp}^{2}$ with an ideal bond angle of $120^{\circ}$. However, in 5-membered rings the bond angle in the parent ring must be reduced to $\sim 108^{\circ}$ and the hybridization at the site of the eventual hydrogen atom loss deviates from pure $\mathrm{sp}^{2}$ character. The cleavage of the $\mathrm{C}-\mathrm{H}$ bond to form the radical always leads to an increase in the bond angle at the radical site. For 5-membered rings, a greater change in radical bond angle is correlated with a lower C-H BDE. Stated another way, the 5-membered ring radical is more stable when the radical bond angle approaches that of pure $\mathrm{sp}^{2}$ hybridization.

A general statement can also be made that for both 5- and 6-membered aromatic 
molecules, the radical formed from cleavage of a $\mathrm{C}-\mathrm{H}$ bond becomes more stable as the bond angle at the radical site approaches $120^{\circ}$. For 6-membered rings the angle needs to get smaller in order to approach $120^{\circ}$, whereas a 5-membered ring needs the angle at the nascent radical center to become larger to approach $120^{\circ}$.

The geometric changes upon $\mathrm{C}-\mathrm{H}$ bond cleavage studied are quite regular in the heteroatomic PAHs studied. In each case there is a widening of the internal bond angle at the radical center following loss of the hydrogen atom. In general, the bicyclic molecules that contain a 5-membered ring fused to a 6-membered ring have $\mathrm{C}-\mathrm{H}$ BDEs that are lowest when the change in bond angle at the radical site is greatest. Conversely, fused 6-membered rings exhibit the same behavior as the monocyclic 6-membered rings such that a departure from the ideal $\mathrm{sp}^{2}$ bond angle gives rise to higher $\mathrm{C}-\mathrm{H}$ BDEs.

\subsubsection{1.b. General Trends in Bond Dissociation Enthalpies: Spin Densities}

A further look at the electron distribution within the parent and radical aromatic rings is required to elucidate the trends in C-H BDEs. Previous descriptions of the electronic structure of the radicals formed from $\mathrm{C}-\mathrm{H}$ bond cleavage in aromatic molecules were found to be inaccurate due to the large spin contamination of the UHF wave function. ${ }^{19,54,55}$ For example, the computed $\left\langle S^{2}\right\rangle$ for the phenyl radical at the UHF/6-31G(d) level is $\sim 1.4$. It has been demonstrated, however, that DFT considerably reduces spin contamination in aryl radicals. ${ }^{24,25,45}$ The computed $\left\langle S^{2}\right\rangle$ values for all of the radicals studied here at the B3LYP/6-31G(d) level are very close to the expected value of 0.75 (Table 3.1.3).

The spin densities of the radicals were evaluated via Bader's Theory of Atoms in Molecules (AIM) $)^{39,41,42}$ at the B3LYP/6-311+G(d,p)//B3LYP/6-31G(d) level. From these calculations, the excess spin density $(\alpha-\beta)$ at each radical site was determined. The formation of the phenyl radical from benzene will again be used to gauge the stability of the radical with respect to spin density. The calculated AIM spin density in the phenyl radical predicts that the unpaired electron is almost completely localized $(\alpha-\beta=0.88)$ at the radical site. A lower C-H 
$\mathrm{BDE}$ is expected when the unpaired spin is not localized on one atomic center, but instead is delocalized over more atomic centers, which stabilizes the radical. By this argument, the spin density population for the radical centers formed via hydrogen atom loss from a carbon neighboring a nitrogen in the azabenzenes should be lower than the spin density population of benzene. The pyridyl radicals provide an example of this prediction. The AIM calculations predict that the spin density at the radical site in 2-pyridyl is 0.75 , with an excess spin density of 0.15 on the nitrogen atom, indicating the delocalization of the radical within the aromatic ring. On the other hand, the C-H BDEs of the 3- and 4-pyridyl radicals were calculated to be very similar to the $\mathrm{C}-\mathrm{H}$ BDE of benzene, and the AIM calculations support this finding with spin densities at the radical site of 0.86 and 0.85 , respectively.

In general, there is a correlation between the spin density population and the C-H BDE, and the correlation is independent of the number of atoms in the aromatic ring. The spin density populations can also be used to understand the relative stability of the radicals formed from the same parent molecule as well as between radicals formed from aromatic molecules of different ring size. This analysis suggests that there is less sharing of the excess spin density in the 5membered rings. As a result of the localization of the radical density, 5-membered rings form less stable radicals than 6-membered rings, resulting in higher C-H BDEs. This supports the trends observed in the change in geometry. The AIM spin density calculations of the PAHs show that, in general, cleavage of a $\mathrm{C}-\mathrm{H}$ bond leaves the odd electron localized at the radical center. Therefore, extension of the $\pi$ system in the PAHs has little effect on the strength and electronic structure of the $\sigma \mathrm{C}-\mathrm{H}$ bonds. The resulting radicals formed at carbon centers that neighbor nitrogen in the bicyclic molecules such as quinoline and isoquinoline also show a sharing of the radical spin with the nitrogen and a lower C-H BDE, similar to the radicals formed in pyridine and the diazines. 
Table 3.1.3. The Calculated Electronic and Geometric Properties of Monocyclic Aromatic Radicals

\begin{tabular}{lccccc}
\hline \multicolumn{1}{c}{ Parent } & Site $^{a}$ & $\left\langle\mathrm{~S}^{2}\right\rangle^{b}$ & $\alpha-\beta^{c}$ & angle change ${ }^{b, d}$ & C-H BDE $^{b, e}$ \\
\hline benzene & 1 & 0.7575 & 0.88 & 5.7 & 110.8 \\
pyridine & 2 & 0.7551 & 0.75 & 2.8 & 105.6 \\
& 3 & 0.7575 & 0.86 & 5.2 & 111.3 \\
pyridazine & 4 & 0.7567 & 0.85 & 5.3 & 110.0 \\
& 3 & 0.7550 & 0.74 & 2.9 & 107.0 \\
pyrimidine & 4 & 0.7569 & 0.77 & 4.2 & 108.9 \\
& 2 & 0.7551 & 0.77 & 3.8 & 107.2 \\
pyrazine & 4 & 0.7545 & 0.73 & 2.5 & 104.7 \\
furan & 5 & 0.7574 & 0.85 & 5.0 & 112.2 \\
& 2 & 0.7552 & 0.72 & 1.7 & 105.7 \\
thiophene & 3 & 0.7601 & 0.89 & 3.4 & 118.4 \\
& 2 & 0.7574 & 0.91 & 3.6 & 118.3 \\
pyrrole & 3 & 0.7611 & 0.91 & 4.0 & 116.6 \\
& 2 & 0.7581 & 0.87 & 5.1 & 113.8 \\
oxazole & 3 & 0.7585 & 0.89 & 3.5 & 118.1 \\
& 2 & 0.7568 & 0.91 & 3.5 & 117.6 \\
& 4 & 0.7580 & 0.82 & 3.3 & 117.8 \\
& 5 & 0.7571 & 0.86 & 3.0 & 119.9 \\
\hline
\end{tabular}

The radical site.

b B3LYP/6-31G(d) level of theory

c Spin density population at radical site determined by Bader's Theory of Atoms in Molecules at the B3LYP/6-311+G(d,p)//B3LYP/6-31G(d) level of theory.

d Bond angle widening upon $\mathrm{H}$ atom loss defined as radical - parent, in degrees.

- $\quad \mathrm{BDE}$ at $298 \mathrm{~K}$ in $\mathrm{kcal}-\mathrm{mol}^{-1}$ 


\subsubsection{Temperature Effects on C-H BDEs.}

The $\mathrm{C}-\mathrm{H}$ BDEs presented in this study provide an understanding of the preference for $\mathrm{H}$ atom abstraction from small aromatic hydrocarbons at $298 \mathrm{~K}$. However, the temperatures in coal combustion processes can fall in a typical range of 500 to $1500 \mathrm{~K}$. To demonstrate whether 298 $\mathrm{K}$ BDE calculations are relevant at temperatures well above $298 \mathrm{~K}$, the energy required to cleave a C-H bond in benzene, furan, and pyridine were calculated in the temperature range from $300 \mathrm{~K}$ to $2000 \mathrm{~K}$ for the formation of the most stable radical for each. The effect of temperature on the calculated C-H BDEs evaluated at the B3LYP level is small, with a variation of only $\sim 2.5 \mathrm{kcal}-$ $\mathrm{mol}^{-1}$ between room temperature and $1200 \mathrm{~K}$. Therefore, accurate conclusions with respect to coal combustion processes can be expected when $\mathrm{C}-\mathrm{H}$ bond strengths calculated at $298 \mathrm{~K}$ are used.

\subsubsection{PAHs can be approximated by Monocyclic Aromatic Compounds.}

The $\mathrm{C}-\mathrm{H}$ bond dissociation enthalpies of the monocyclic aromatic hydrocarbons calculated via B3LYP/6-31G(d) reproduce quite well the available experimental C-H BDEs for these rings. However, to gauge whether the monocyclic aromatic hydrocarbons can adequately describe the initial processes of combustion in coal, calculations were also performed on representative polycyclic rings that can begin to model the extended carbon network. In general, monocyclic BDE calculations can be used to estimate the $\mathrm{C}-\mathrm{H} \mathrm{BDE}$ of the polycyclic

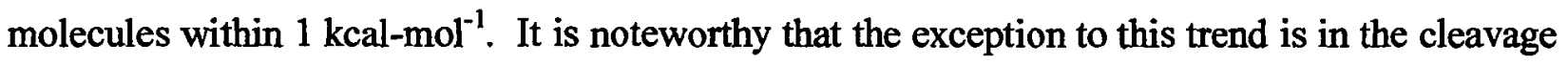
of the $\mathrm{C}-\mathrm{H}$ bond that is adjacent to a ring junction where the ring junction is also adjacent to a heteroatom. At this position, the C-H BDE can vary by as much as $2.4 \mathrm{kcal}^{-\mathrm{mol}^{-1}}$ as in the case

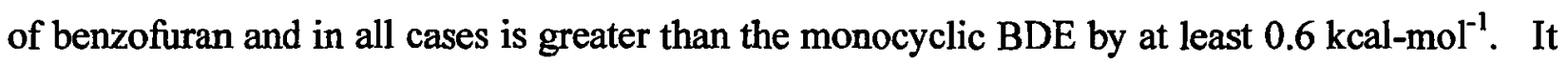
appears that a more electronegative heteroatom yields a larger difference between the polycyclic and monocyclic ring, as the $\mathrm{C}-\mathrm{H}$ BDE of benzothiophene at the 7 position has a very similar $\mathrm{C}-\mathrm{H}$ BDE to that of benzene. 


\subsubsection{Conclusions}

The B3LYP level of theory is a useful method to predict the properties and BDEs for aromatic hydrocarbons. The agreement of C-H BDEs predicted by B3LYP with the available experimental values for the small aromatic molecules provides confidence that the B3LYP method is well-suited to the study of larger aromatic molecules. In addition, reliable C-H BDEs can be obtained with even a very modest basis set, $6-31 \mathrm{G}(\mathrm{d})$, because the $\mathrm{C}-\mathrm{H}$ bond is non-polar.

This computational study has endeavored to study specific bonds, $\mathrm{C}-\mathrm{H}$ and $\mathrm{N}-\mathrm{H}$, within a coal matrix to gain an understanding of the chemical reactivity of coal. To that end the $\mathrm{C}-\mathrm{H}$ and $\mathrm{N}-\mathrm{H}$ BDEs of several monocyclic and polycyclic aromatic molecules were studied, including molecules that contain the heteroatoms $\mathrm{O}, \mathrm{S}$, and $\mathrm{N}$. The cleavage of a $\mathrm{C}-\mathrm{H}$ or a $\mathrm{N}-\mathrm{H}$ bond generates a $\sigma$ radical that, in general, is localized at the radical site. The $\mathrm{C}-\mathrm{H}$ bond adjacent to nitrogen in the azabenzenes is predicted to be $\sim 7 \mathrm{kcal}^{-\mathrm{mol}^{-1}}$ weaker than the $\mathrm{C}-\mathrm{H}$ bond in benzene due to delocalization of the unpaired electron. Both localization of the spin density and geometric factors following cleavage of a $\mathrm{C}-\mathrm{H}$ bond contribute to the stability of the radicals formed. Therefore, 5-membered ring radicals are $\sim 6 \mathrm{kcal}^{-\mathrm{mol}^{-1}}$ less stable than those formed from 6-membered aromatic molecules. We, therefore, predict that in coal the $\mathrm{C}-\mathrm{H}$ bonds on any 5-membered ring will be significantly less reactive than those on 6-membered rings. The location of the heteroatoms in the aromatic ring affects the $\mathrm{C}-\mathrm{H}$ bond strengths more significantly than the size of the aromatic network. Therefore, the monocyclic aromatic molecules are good models to study the cleavage of aromatic $\mathrm{C}-\mathrm{H}$ bonds in coal and can predict the $\mathrm{C}-\mathrm{H} \mathrm{BDE}$ of the polycyclic molecules within $1 \mathrm{kcal}-\mathrm{mol}^{-1}$. However, increasing the size of the aromatic network does reduce the strength of any N-H bonds that are present.

The B3LYP method accurately reproduces the available experimental BDEs. Therefore, the B3LYP method is a promising method to gain further computational insight into the processes of coal combustion. 


\section{References for Section 3.1.}

1.) Smith, K. L.; Smoot, L. D.; Fletcher, T. H.; Pugmire, R. J. The Structure and Reaction Processes of Coal; Plenum Press: New York, 1994.

2.) Meyers, R. A. Coal Structure; Academic Press: New York, 1982.

3.) Glassman, I. Combustion; Academic Press: San Diego, 1996.

4.) Davico, G. E.; Bierbaum, V. M.; DePuy, C. H.; Ellison, G. B.; Squires, R. R. J. Am. Chem. Soc. 1995, 117, 2590-2599.

5.) Nimmo, W.; Richardson, J.; Hampartsoumian, E. J. Inst. Energy 1995, 68, 170-177.

6.) Williams, A.; Pourkashanian, M.; Jones, J. M.; Rowlands, L. J. Inst. Energy 1997, 70, 102113.

7.) Mackie, J. C.; Colket, M. B., III; Nelson, P. F. J. Phys. Chem. 1990, 94, 4099-4106.

8.) Doughty, A.; Mackie, J. C. J. Chem. Soc. Faraday Trans. 1994, 90, 541-548.

9.) Kiefer, J. H.; Zhang, Q.; Kern, R. D.; Yao, J.; Jursic, B. J. Phys. Chem. A 1997, 101, 70617073.

10.) Jones, J.; Bacskay, G. B.; Mackie, J. C.; Doughty, A. J. Chem. Soc. Faraday Trans. 1995, 91, 1587-1592.

11.) Blank, D. A.; North, S. W.; Lee, Y. T. Chem. Phys. 1994, 187, 35-47.

12.) Hudgins, D. M.; Sandford, S. A. J. Phys. Chem. A 1998, 102, 344-352.

13.) Hudgins, D. M.; Sandford, S. A. J. Phys. Chem. A 1998, 102, 329-343.

14.) Chen, R. H.; Kafafi, S. A.; Stein, S. E. J. Am. Chem. Soc. 1989, 111, 1418-1423.

15.) Meot-Ner, M. J. Phys. Chem. 1980, 84, 2716-2723.

16.) Kass, S., University of Minnesota, private communication.

17.) Laskin, A.; Lifshitz, A. J. Phys. Chem. A 1997, 101, 7787-7801.

18.) Laskin, A.; Lifshitz, A. J. Phys. Chem. A 1998, 102, 928-946.

19.) Aihara, J.-I.; Fujiwara, K.; Harada, A.; Ichikawa, H.; Fukushima, K.; Hirota, F.; Ishida, T. J. Mol. Struct. 1996, 366, 219-226.

20.) Hehre, W. J.; Radom, L.; Schleyer, P. v. R.; Pople, J. A. Ab Initio Molecular Orbital

Theory; John Wiley \& Sons: New York, 1986.

21.) Parr, R. G.; Yang, W. Density-Functional Theory of Atoms and Molecules; Oxford

University Press: New York, 1989.

22.) Ziegler, T. Chem. Rev. 1991, 91, 651-667.

23.) Wiberg, K. B. J. Org. Chem. 1997, 62, 5720-5727.

24.) Cioslowski, J.; Liu, G.; Martinov, M.; Piskorz, P.; Moncrieff, D. J. Am. Chem. Soc. 1996, $118,5261-5264$.

25.) Cioslowski, J.; Liu, G.; Moncrieff, D. J. Org. Chem. 1996, 61, 4111-4114.

26.) Johnson, B. G.; Gill, P. M. W.; Pople, J. A. J. Chem. Phys. 1993, 98, 5612-5626.

27.) Lee, C.; Yang, W.; Parr, R. G. Phys. Rev. B 1988, 37, 785-789.

28.) Stephens, P. J.; Devlin, F. J.; Frisch, M. J. J. Phys. Chem. 1994, 98, 11623-11627.

29.) Becke, A. D. J. Chem. Phys. 1993, 98, 5648-5652.

30.) Carpenter, B. K. J. Am. Chem. Soc. 1993, 115, 9806-9807.

31.) Liu, R.; Morokuma, K.; Mebel, A. M.; Lin, M. C. J. Phys. Chem. 1996, 100, 9314-9322.

32.) Madden, L. K.; Moskaleva, L. V.; Kristyan, S.; Lin, M. C. J. Phys. Chem. A 1997, 101, 6790-6797.

33.) Frisch, M. J.; Trucks, G. W.; Schlegel, H. B.; Gill, P. M. W.; Johnson, B. G.; Robb, M. A.; Cheeseman, J. R.; Kieth, T.; Petersson, G. A.; Montgomery, J. A.; Raghavachari, K.; Al-Laham, M. A.; Zakrzewski, V. G.; Ortiz, J. V.; Foresman, J. B.; Cioslowski, J.; Stefanov, B. B.; 
Nanayakkara, A.; Challacombe, M.; Peng, C. Y.; Ayala, P. Y.; Chen, W.; Wong, M. W.; Andres, J. L.; Replogle, E. S.; Gomperts, R.; Martin, R. L.; Fox, D. J.; Binkley, J. S.; Defrees, D. J.; Baker, J.; Stewart, J. P.; Head-Gordon, M.; Gonzalez, C.; Pople, J. A. Gaussian 94; C.3 ed.; Gaussian, Inc.: Pittsburgh, PA, 1995.

34.) Pople, J. A.; Scott, A. P.; Wong, M. W.; Radom, L. Isr J. Chem. 1993, 33, 345-350.

35.) Scott, A. P.; Radom, L. J. Phys. Chem. 1996, 100, 16502-16513.

36.) Ochterski, J. W.; Petersson, G. A.; Wiberg, K. B. J. Am. Chem. Soc. 1995, 117, 11299 11308.

37.) Ochterski, J. W.; Petersson, G. A.; J.A. Montgomery, J. J. Chem. Phys. 1996, 104, 25982619.

38.) Curtiss, L. A.; Raghavachari, K.; Pople, J. A. J. Chem. Phys. 1993, 98, 1293-1298.

39.) Bader, R. F. W. Acc. Chem. Res. 1985, 18, 9-15.

40.) Wiberg, K. B.; Rablen, P. R. J. Comp. Chem. 1993, 14, 1504-1518.

41.) Bader, R. F. W. Chem. Rev. 1991, 91, 893-928.

42.) Bader, R. F. W. Atoms in Molecules - A Quantum Theory; Clarendon Press: Oxford, 1990.

43.) Keith, T. A. AIMALL: Yale University, 1996.

44.) Wiberg, K. B.; Cheeseman, J. R.; Ochterski, J. W.; Frisch, M. J. J. Am. Chem. Soc. 1995, $117,6535-6543$.

45.) Cioslowski, J.; Liu, G.; Moncrieff, D. J. Phys. Chem. A 1997, 101, 957-960.

46.) Nicolaides, A.; Radom, L. Mol. Phys. 1996, 88, 759-765.

47.) Mayer, P. M.; Parkinson, C. J.; Smith, D. M.; Radom, L. J. Chem. Phys. 1998, 108, 604615.

48.) Herzberg, G. H. Molecular Spectra and Molecular Structure: Electronic Spectra and Electronic Structure of Polyatomic Molecules.; Van Nostrand Reinhold Company: New York, 1967; Vol. III.

49.) Berkowitz, J.; Ellison, G. B.; Gutman, D. J. Phys. Chem. 1994, 98, 2744-2765.

50.) Organ, P. P.; Mackie, J. C. J. Chem. Soc. Faraday Trans. 1991, 87, 815-823.

51.) Lifshitz, A.; Bidani, M.; Bidani, S. J. Phys. Chem. 1986, 90, 5373-5377.

52.) Lifshitz, A.; Tamburu, C.; Suslensky, A. J. Phys. Chem. 1989, 93, 5802-5808.

53.) Bordwell, F. G.; Zhang, X.; Cheng, J.-P. J. Org. Chem. 1991, 56, 3216-3219.

54.) Pacansky, J.; Liu, B.; DeFrees, D. J. Org. Chem. 1986, 51, 3720-3721.

55.) Hameka, H. F. J. Org. Chem. 1987, 52, 5025-5026. 


\subsection{Computational Study of the Unimolecular Decomposition Pathways of Phenylperoxy Radical}

\subsubsection{Executive Summary}

The potential energy surface for the unimolecular decomposition of phenylperoxy radical has been explored using the B3LYP method. Several pathways were considered including the initial formation of the phenoxy, dioxiranyl, 1,2-dioxetanyl, 1,3-peroxy and $p$-phenylquinone radicals. Transition states for all pathways on the potential energy surface are presented. At all temperatures studied ( $\mathrm{T} \leq 1250 \mathrm{~K}$ ), the energetically most favored pathway is the dioxiranyl pathway which leads to the formation of cyclopentadienyl radical and $\mathrm{CO}_{2}$, pyranyl radical and $\mathrm{CO}$, or an acyclic $\mathrm{C}_{6} \mathrm{H}_{5} \mathrm{O}_{2}$ radical structure as products. The ring-opening reactions are very competitive with formation of $\mathrm{CO}$ and $\mathrm{CO}_{2}$ as products.

\subsubsection{Introduction}

The reaction of phenyl radical with $\mathrm{O}_{2}$ is of great importance in the study of combustion and atmospheric chemistry and has received much attention. The major product channel at intermediate and high temperatures has been reported to follow this path: $:^{1-3}$

$$
\begin{aligned}
& \mathrm{C}_{6} \mathrm{H}_{5} \bullet+\mathrm{O}_{2} \rightarrow \mathrm{C}_{6} \mathrm{H}_{5} \mathrm{O} \bullet+\mathrm{O} \\
& \mathrm{C}_{6} \mathrm{H}_{5} \mathrm{O} \bullet \rightarrow \mathrm{C}_{5} \mathrm{H}_{5} \bullet+\mathrm{CO}
\end{aligned}
$$

but controversy still exists over the exact mechanisms as current models still fail to accurately predict the experimental formation and concentrations of products at various temperatures from the combustion of benzene with oxygen. ${ }^{4}$

The relevance of phenylperoxy radical $\left(\mathrm{C}_{6} \mathrm{H}_{5} \mathrm{OO} \bullet\right)$ as a viable intermediate at intermediate temperatures is also disputed. Benson theorized on thermochemical grounds that phenylperoxy radical would not be stable at temperatures above $433 \mathrm{~K},{ }^{5}$ but it has been shown experimentally that at temperatures as high as $473 \mathrm{~K}, \mathrm{C}_{6} \mathrm{H}_{5} \mathrm{OO} \cdot$ can still be detected and the phenoxy radical $\left(\mathrm{C}_{6} \mathrm{H}_{5} \mathrm{O}^{\bullet}\right)$ is absent. ${ }^{6}$ Therefore, $\mathrm{C}_{6} \mathrm{H}_{5} \mathrm{OO} \bullet$ cannot be discounted as an important 
intermediate of the reaction of phenyl radical with oxygen at low to intermediate temperatures.

Computational studies over the past decade have explored alternate pathways of the unimolecular decomposition of $\mathrm{C}_{6} \mathrm{H}_{5} \mathrm{OO} \bullet$ and have centered mainly on the formation of dioxiranyl and dioxetanyl intermediates which can then decompose to the cyclopentadienyl radical $\left(\mathrm{C}_{5} \mathrm{H}_{5}{ }^{\bullet}, \mathrm{Cp}^{\bullet}\right)$ and $\mathrm{CO}$ or $\mathrm{CO}_{2} .^{7-8}$ The dioxiranyl pathway was proposed by Carpenter based on PM3 calculations. ${ }^{7}$ Further analysis at the B3LYP/6-31G* level demonstrated the viability of the dioxiranyl pathway at low temperatures; however, we were unable at that time to provide a complete potential energy surface, including activation barriers, for the decomposition of aromatic peroxy radicals. ${ }^{8}$ These previous studies suggested that other pathways may be competitive with the phenoxy radical pathway at intermediate temperatures $(\mathrm{T}<1000 \mathrm{~K}) .^{7-8}$

The vinyl radical and $\mathrm{O}_{2}$ potential energy surface has been exhaustively studied by Carpenter ${ }^{9}$ and Lin, Morokuma and co-workers, ${ }^{10}$ but larger, unsaturated radicals have been less well studied. However, there have been some computational studies relevant to phenyl radical oxidation. ${ }^{11-14}$ For instance, the decomposition pathways for the phenoxy radical have been investigated. ${ }^{11,12}$ Various $\mathrm{C}_{6} \mathrm{H}_{5} \mathrm{O}_{2}$ isomers have been studied by Mebel and $\mathrm{Lin}^{13}$ and these authors also examined the $\mathrm{O}+\mathrm{C}_{6} \mathrm{H}_{5} \mathrm{O} \bullet$ potential energy surface. ${ }^{14}$ However, a quantitatively accurate comparison of the different pathways available to the phenylperoxy radical is still lacking. Some of the previous computational studies were completed via unrestricted HartreeFock based methods, and spin contamination was significant. Lin, Morokuma and co-workers have shown that density functional theory methods can be accurately applied to benzene reactions with hydrogen atoms. ${ }^{15}$ We have also demonstrated that DFT methods, and the B3LYP functional in particular, can be accurately applied to aromatic radicals as spin contamination is not a significant problem. ${ }^{8,16}$

This paper is an extension of a previous computational study of the possible unimolecular decomposition pathways of $\mathrm{C}_{6} \mathrm{H}_{5} \mathrm{OO} \cdot$ at temperatures ranging from 273 to $2000 \mathrm{~K}$. Computationally, we now explore the phenoxy and dioxiranyl radical pathways as well as the 1,2-dioxetanyl, 1,3-peroxy and $p$-phenylquinonyl radical pathways and provide energetics of the 
intermediates and transition states using the B3LYP density functional theory method.

\subsubsection{Computational Methods}

All geometry optimizations and vibrational frequency calculations were performed with Gaussian 94 and 98 at the Ohio Supercomputer Center. ${ }^{17,18}$ The optimized geometries were calculated at the B3LYP/6-31G* hybrid density functional theory level and single point energies at these optimized geometries were determined at the B3LYP/6-311+G** level (using the scf-tight option). ${ }^{19-22}$ All basis sets used 6 Cartesian $d$ functions. Bauschlicher and Langhoff have shown that there is a small basis set effect in B3LYP calculations for oxygen containing systems for determining $\mathrm{C}-\mathrm{H}$ bond dissociation energies. ${ }^{23}$ The B3LYP/6-311+G** single point energies should compensate for such deficiencies.

In order to compare our B3LYP data to other theoretical levels, we have also determined single point energies at the CASSCF(7,8)/6-31G*, CAS-MP2(7,8)/6-31G* and UCCSD(T)/6$31 \mathrm{G}^{* *}$ levels for some key intermediates and transition states. ${ }^{24-28}$ The active space for the CASSCF calculations was problematic to determine for the disparate structures, as most of them were of $C_{1}$ symmetry. For $1\left(C_{s}\right.$ symmetry), 6 orbitals were of $\pi$ symmetry, and the 2 chosen $\sigma$ orbitals had large $\mathrm{O}-\mathrm{O}$ interactions. For the other structures, the 4 highest occupied and 4 lowest unoccupied orbitals were used as obtained from $\mathrm{ROHF} / 6-31 \mathrm{G}^{*}$ wave functions. The CASSCF and UCCSD(T) calculations were completed with Gaussian 98.

The stationary points were verified to be minima or saddle points via vibrational frequency analyses. All transition states were confirmed to connect to reactants and products by incrementally displacing (typically $10 \%$ ) the geometries along the reaction coordinate for the imaginary vibrational frequency in either direction, calculating the analytical force constants, and then optimizing to the corresponding minimum or by using an intrinsic reaction coordinate (IRC) search. $^{29,30}$

Spin contamination throughout this study was very minimal. The $\left\langle S^{2}\right\rangle$ values for the doublet species (minima and transition states) in this paper were $\leq 0.80$, with only six that 
exceeded 0.80. Three of the six possess minimal spin contamination (TS 1-24, 0.88; TS 24-25, 0.84; TS 1-28, 0.82), while the other three suffer from extreme spin contamination (TS 1-3, 1.77;

TS 2-1, 1.76; 9, 1.82) causing the energetics calculated for these molecules to be greatly suspect.

The thermodynamic contribution to the free energy of each molecule was determined

from the unscaled vibrational frequencies using the Thermo94 program. ${ }^{31}$ The overall Gibbs free energy at each temperature was calculated by adding the single point energy, the scaled zero point energy, the thermodynamic contribution to the free energy and the electronic contribution to the Gibbs free energy.

$$
\mathrm{G}(\mathrm{T})=\mathrm{E}_{\mathrm{SP}}+\mathrm{ZPE}+\mathrm{G}_{\mathrm{Thermo}}+\mathrm{RT} \ln 2
$$

A scaling factor of 0.9806 was used for the ZPE as determined at the B3LYP/6-31G* level. $^{32}$ The only molecule which was treated differently was $\mathrm{O}$ atom, where experimentally ${ }^{33}$ determined splitting energies were included in the free energy calculations to determine the electronic component of the partition function.

The energies discussed below are free energies computed at the B3LYP/6311+G**//B3LYP/6-31G*, unless noted otherwise. The energies have been evaluated from $298 \mathrm{~K}$ to $1250 \mathrm{~K}$ and will be discussed accordingly in the text.

\subsubsection{Results and Discussion}

Formation of $\mathrm{C}_{6} \mathrm{H}_{5} \mathrm{OO} \cdot$. Formation of $\mathrm{C}_{6} \mathrm{H}_{5} \mathrm{OO} \bullet$ (1) from the oxidation of phenyl radical with molecular oxygen (2) is exoergic at temperatures ranging from 298 to $1000 \mathrm{~K}$ and endoergic at temperatures above $1250 \mathrm{~K}$ (Table 3.2.1). The B3LYP/6-311+G**//B3LYP/6-31G* method calculates this reaction to be favorable by $-32.3 \mathrm{kcal} / \mathrm{mol}$ at $298 \mathrm{~K}$, decreasing steadily to only $-7.1 \mathrm{kcal} / \mathrm{mol}$ at $1000 \mathrm{~K}$ and becoming unfavorable by $+1.6 \mathrm{kcal} / \mathrm{mol}$ at $1250 \mathrm{~K}$. The transition state (TS 2-1) for this reaction has been found, but suffers from excessive spin 
Table 3.2.1. Gibbs Free Energy for all intermediates and transition states (298-1250K) at the B3LYP/6-311+G**//B3LYP/6-31G* level.

\begin{tabular}{|c|c|c|c|c|c|c|c|}
\hline Structure & $\begin{array}{c}E^{9} \\
\text { (hartrees/part) }\end{array}$ & $\begin{array}{c}\Delta G(298 \mathrm{~K}) \\
(\mathrm{kcal} / \mathrm{mol})\end{array}$ & $\begin{array}{l}\Delta G(500 \mathrm{~K}) \\
(\mathrm{kcal} / \mathrm{mol})\end{array}$ & $\begin{array}{l}\Delta \mathrm{G}(750 \mathrm{~K}) \\
(\mathrm{kcal} / \mathrm{mol})\end{array}$ & $\begin{array}{c}\Delta G(1000 \mathrm{~K}) \\
(\mathrm{kcal} / \mathrm{mol})\end{array}$ & $\begin{array}{c}\Delta G(1250 \mathrm{~K}) \\
(\mathrm{kcal} / \mathrm{mol})\end{array}$ & $\left\langle S^{2}\right\rangle$ \\
\hline 1 & -382.06960 & 0.0 & 0.0 & 0.0 & 0.0 & 0.0 & 0.76 \\
\hline TS 1-3 & -381.98196 & 51.0 & 49.8 & 48.3 & 46.8 & 45.5 & 1.77 \\
\hline TS 1-8 & -382.02404 & 27.2 & 27.6 & 28.0 & 28.6 & 29.3 & 0.79 \\
\hline TS 1-19 & -381.99465 & 46.2 & 46.9 & 47.9 & 49.0 & 50.2 & 0.78 \\
\hline TS 1-24 & -381.90221 & 102.8 & 103.5 & 104.3 & 105.1 & 106.0 & 0.88 \\
\hline TS 1-28 & -381.92072 & 91.4 & 91.9 & 92.6 & 93.3 & 94.0 & 0.82 \\
\hline $2^{a}$ & -381.99718 & 32.3 & 24.9 & 15.9 & 7.1 & -1.6 & 0.76 \\
\hline TS 2-1 & -381.99742 & 38.4 & 34.9 & 30.7 & 26.6 & 22.6 & 1.76 \\
\hline $3^{b}$ & -382.00773 & 27.3 & 20.6 & 12.3 & 3.9 & -4.4 & 0.79 \\
\hline TS $3-4^{b}$ & -381.92354 & 77.8 & 71.1 & 62.8 & 54.6 & 46.4 & 0.77 \\
\hline TS 3-6 & -381.88861 & 96.8 & 88.5 & 77.9 & 67.4 & 57.0 & 0.77 \\
\hline $4^{b}$ & -381.92831 & 75.1 & 68.0 & 58.9 & 48.9 & 40.6 & 0.77 \\
\hline TS 4-5 & -381.91606 & 82.5 & 75.6 & 67.1 & 58.7 & 50.3 & 0.77 \\
\hline $5^{b}$ & -381.94726 & 62.5 & 54.7 & 44.6 & 34.4 & 24.2 & 0.75 \\
\hline TS 5-7 & -381.93812 & 66.6 & 58.4 & 48.1 & 37.9 & 27.7 & 0.76 \\
\hline $6^{b}$ & -381.90079 & 89.2 & 80.2 & 68.7 & 57.0 & 45.3 & 0.77 \\
\hline TS $6-5^{b}$ & -381.88335 & 100.5 & 92.5 & 82.3 & 72.2 & 62.2 & 0.78 \\
\hline $7^{c}$ & -381.95912 & 41.1 & 20.6 & -3.1 & -27.3 & -52.0 & 0.77 \\
\hline 8 & -382.03201 & 22.6 & 22.8 & 22.9 & 22.9 & 22.9 & 0.78 \\
\hline TS 8-9 & -382.01201 & 33.9 & 33.9 & 33.9 & 34.0 & 34.2 & 0.76 \\
\hline TS 8-10 & -381.91393 & 92.6 & 91.7 & 90.4 & 89.1 & 87.8 & 0.78 \\
\hline TS 8-15 & -381.95475 & 69.3 & 69.6 & 69.9 & 70.2 & 70.7 & 0.78 \\
\hline 9 & -382.00039 & 41.6 & 41.6 & 41.4 & 41.1 & 40.7 & 1.82 \\
\hline TS 9-10 & -382.01759 & 31.1 & 31.5 & 32.0 & 32.7 & 33.4 & 0.76 \\
\hline 10 & -382.14542 & -48.1 & -48.4 & -48.8 & -49.2 & -49.6 & 0.78 \\
\hline TS 10-11 & -382.08120 & -8.6 & -8.3 & -7.8 & -7.2 & -6.5 & 0.80 \\
\hline TS 10-17 & -382.06345 & 5.7 & 5.1 & 4.5 & 3.9 & 3.4 & 0.75 \\
\hline TS 10-21 & -382.10117 & -24.7 & -26.4 & -28.7 & -30.8 & -32.8 & 0.78 \\
\hline 11 & -382.13066 & -38.1 & -37.7 & -37.1 & -36.6 & -36.2 & 0.78 \\
\hline TS 11-12 & -382.11495 & -29.4 & -29.1 & -28.5 & -27.8 & -27.0 & 0.77 \\
\hline TS 11-13 & -382.09064 & -14.5 & -14.2 & -13.8 & -13.3 & -12.7 & 0.80 \\
\hline 12 & -382.11603 & -30.8 & -31.3 & -32.0 & -32.9 & -33.7 & 0.76 \\
\hline TS 12-14 & -382.11593 & -31.5 & -31.9 & -32.2 & -32.5 & -32.7 & 0.76 \\
\hline 13 & -382.09894 & -20.1 & -21.0 & -22.2 & -23.4 & -24.6 & 0.75 \\
\hline TS 13-14 & -382.07261 & -4.4 & -4.9 & -5.5 & -6.0 & -6.4 & 0.76 \\
\hline $14^{d}$ & -382.16688 & -76.1 & -89.4 & -104.0 & -119.3 & -134.9 & 0.77 \\
\hline 15 & -381.96804 & 62.1 & 62.2 & 62.3 & 62.2 & 62.1 & 0.77 \\
\hline TS 15-12 & -381.95538 & 68.9 & 69.2 & 69.6 & 70.0 & 70.4 & 0.76 \\
\hline 16 & -381.92550 & 84.9 & 82.8 & 79.8 & 76.7 & 73.5 & 0.77 \\
\hline 17 & -382.09779 & -19.1 & -19.9 & -20.9 & -21.9 & -22.9 & 0.76 \\
\hline
\end{tabular}




\begin{tabular}{|c|c|c|c|c|c|c|c|}
\hline TS 17-18 & -382.09552 & -18.9 & -19.6 & -20.5 & -21.3 & -22.0 & 0.77 \\
\hline $18^{\circ}$ & -382.12348 & -47.9 & -55.6 & -65.2 & -74.6 & -83.9 & 0.78 \\
\hline 19 & -381.99722 & 44.8 & 45.2 & 45.6 & 46.0 & 46.3 & 0.78 \\
\hline TS $19-20$ & -381.99633 & 44.5 & 45.1 & 45.8 & 46.7 & 47.6 & 0.80 \\
\hline 20 & -382.09519 & -18.0 & -18.4 & -19.1 & -19.8 & -20.6 & 0.76 \\
\hline TS 20-10 & -382.07785 & -6.9 & -6.7 & -6.5 & -6.1 & -5.6 & 0.77 \\
\hline TS 20-21 & -382.09181 & -16.7 & -17.0 & -17.3 & -17.5 & -17.7 & 0.76 \\
\hline 21 & -382.11734 & -34.0 & -36.0 & -38.7 & -41.4 & -44.2 & 0.78 \\
\hline TS 21-22 & -382.10567 & -27.2 & -28.8 & -30.8 & -32.7 & -34.5 & 0.78 \\
\hline 22 & -382.12212 & -37.2 & -39.5 & -42.4 & -45.5 & -48.5 & 0.78 \\
\hline TS 22-23 & -382.10755 & -28.6 & -30.3 & -32.5 & -34.6 & -36.6 & 0.78 \\
\hline 23 & -382.12971 & -40.8 & -42.3 & -44.4 & -46.5 & -48.6 & 0.77 \\
\hline 24 & -381.93351 & 84.0 & 84.6 & 85.2 & 85.7 & 86.1 & 0.79 \\
\hline TS 24-25 & -381.93412 & 82.6 & 83.4 & 84.3 & 85.3 & 86.2 & 0.84 \\
\hline 25 & -382.11072 & -25.8 & -25.5 & -25.2 & -24.8 & -24.5 & 0.78 \\
\hline TS 25-26 & -382.08708 & -12.1 & -11.7 & -11.1 & -10.3 & -9.4 & 0.79 \\
\hline 26 & -382.11026 & -25.9 & -26.2 & -26.6 & -27.0 & -27.4 & 0.77 \\
\hline TS 26-27 & -382.05548 & 6.9 & 7.1 & 7.3 & 7.6 & 7.9 & 0.77 \\
\hline 27 & -382.06854 & -0.5 & -0.6 & -0.9 & -1.2 & -1.6 & 0.77 \\
\hline TS 27-17 & -382.04945 & 10.6 & 10.6 & 10.7 & 10.9 & 11.1 & 0.79 \\
\hline 28 & -382.09630 & -17.9 & -18.1 & -18.4 & -18.8 & -19.1 & 0.75 \\
\hline TS 28-29 & -382.02212 & 28.1 & 28.8 & 29.7 & 30.6 & 31.6 & 0.76 \\
\hline 29 & -382.03599 & 20.4 & 21.1 & 21.8 & 22.4 & 22.9 & 0.76 \\
\hline TS 29-30 & -382.03190 & 22.1 & 22.9 & 23.9 & 25.0 & 26.1 & 0.77 \\
\hline 30 & -382.08591 & -12.4 & -13.1 & -14.1 & -15.2 & -16.3 & 0.76 \\
\hline TS $30-31$ & -382.05549 & 3.7 & 2.3 & 0.4 & -1.6 & -3.5 & 0.78 \\
\hline TS $30-32$ & -382.05363 & 8.8 & 9.4 & 10.3 & 11.2 & 12.2 & 0.79 \\
\hline $31^{f}$ & -382.08322 & -23.5 & -31.4 & -41.3 & -51.2 & -61.0 & 0.77 \\
\hline 32 & -382.09249 & -13.5 & -12.7 & -11.6 & -10.6 & -9.6 & 0.75 \\
\hline TS 32-12 & -382.08620 & -10.8 & -10.0 & -8.9 & -7.7 & -6.4 & 0.76 \\
\hline
\end{tabular}

${ }^{a}$ Energies include the aryl species and $\mathrm{O}_{2} ;\left\langle\mathrm{S}^{2}\right\rangle$ values and symmetry are of the aryl species only.

${ }^{b}$ Energies include the aryl species and $O_{;}\left\langle S^{2}\right\rangle$ values and symmetry are of the aryl species only.

'Energies include the aryl species, $\mathrm{O}$ and $\mathrm{CO} ;\left\langle\mathrm{S}^{2}\right\rangle$ values and symmetry are of the aryl species only.

${ }^{d}$ Energies include the aryl species and $\mathrm{CO}_{2} ;\left\langle\mathrm{S}^{2}\right\rangle$ values and symmetry are of the aryl species only.

${ }^{e}$ Energies include the aryl species and $\mathrm{CO} ;\left\langle\mathrm{S}^{2}\right\rangle$ values and symmetry are of the aryl species only.

${ }^{f}$ Energies include the aryl species and acetylene ; $\left\langle S^{2}\right\rangle$ values and symmetry are of the aryl species only.

${ }^{9}$ Bottom-of-the-well energy. 
contamination $\left(\left\langle S^{2}\right\rangle=1.76\right)$. This is not surprising, since the reactants are a doublet 2 and a triplet $\mathrm{O}_{2}$, which produce a doublet 1. The energy values for the activation barrier predict a $\Delta \mathrm{G}^{\neq}(298 \mathrm{~K})$ of $6.1 \mathrm{kcal} / \mathrm{mol}$ and increases steadily to a $\Delta \mathrm{G}^{\natural}(1250 \mathrm{~K})$ of $23.2 \mathrm{kcal} / \mathrm{mol}$. The bottom-of-the-well energy value of phenyl radical and $\mathrm{O}_{2}$ compared to the analogous value of TS 2-1 differs by only $0.1 \mathrm{kcal} / \mathrm{mol}$. The activation barriers are higher than expected when compared to the related vinyl peroxy radical formation in which the reaction has been reported to be barrierless at temperatures ranging from 299 to $1005 \mathrm{~K} .{ }^{10,34}$ This discrepancy is probably due to the spin contamination.

The unimolecular decomposition of the phenylperoxy radical (1) was explored along five pathways, denoted by the formation of the subsequent intermediates: phenoxy radical (3) [Path I]; dioxiranyl radical (8) [Path II]; 1,2-dioxetanyl radical (19) [Path III]; 1,3-peroxy radical (24) [Path IV]; and p-phenylquinone radical (28) [Path V]. A complete summary of all the intermediates included in this study is presented in Table 3.2.1. It can be seen that the formation of the final products of $\mathrm{Cp}, \mathrm{O}$ and $\mathrm{CO} ; \mathrm{Cp}^{\bullet}$ and $\mathrm{CO}_{2}$; pyranyl radical (18) and $\mathrm{CO}$; oxyfuranyl radical (31) and acetylene; or an acyclic $\mathrm{C}_{6} \mathrm{H}_{5} \mathrm{O}_{2} \bullet$ species (23) is complicated and several of the products can arise from more than one initial pathway. Experimentally, each of these products, except 23 and 31, have been detected by mass spectrometric studies during benzene oxidation in a microjet reactor at temperatures from 800 to $1300 \mathrm{~K} .^{35}$ The absence of 23 and 31 in these experimental results may be due to their facile fragmentation or further decomposition.

The relative free energies (298K) at the B3LYP/6-311+G**//B3LYP/6-31G* level have been calculated for each intermediate relative to phenylperoxy radical (1) as $0 \mathrm{kcal} / \mathrm{mol}$. For each step, the free energy of activation $\left(\Delta \mathrm{G}^{\neq}, 298 \mathrm{~K}\right)$ is listed relative to the reactant for that specific step. Activation barriers and intermediates which are considered to be suspect (due to spin contamination issues) are shown in parentheses. Table 3.2.1 lists the relative free energies of all stationary points at different temperatures. 
Path I - Phenoxy Radical Intermediate. The phenoxy radical (3) can possibly be produced from the direct reaction of phenyl radical with $\mathrm{O}_{2}$ to form 3 or from the homolysis of the $\mathrm{O}-\mathrm{O}$ bond in phenylperoxy radical (1). The first reaction is exoergic by approximately -3 to $5 \mathrm{kcal} / \mathrm{mol}$ across the temperature range of 298 to $1250 \mathrm{~K}$. On the other hand, the scission of the $\mathrm{O}-\mathrm{O}$ bond from phenylperoxy radical is endoergic by $+27.3 \mathrm{kcal} / \mathrm{mol}$ at $298 \mathrm{~K}$, decreases to +3.9 $\mathrm{kcal} / \mathrm{mol}$ at $1000 \mathrm{~K}$ and becomes exoergic by $-4.4 \mathrm{kcal} / \mathrm{mol}$ at $1250 \mathrm{~K}$. We have attempted to find transition states for both pathways, but without success at the B3LYP/6-31G* level for the first route and obtained energy values $(\sim 51.0 \mathrm{kcal} / \mathrm{mol})$ which are unreliable because of excessive spin contamination for the second route.

We therefore continued to examine the pathways available to the phenoxy radical. Computational exploration of the decomposition pathways of phenoxy radical that were first proposed by Colussi et al. ${ }^{3}$ and a subset of the pathways explored by Morokuma et al. ${ }^{12}$ yielded results that correlate well with the available experimental and computational data. ${ }^{3,12,36}$ For the decomposition of phenoxy radical to $\mathrm{Cp} \bullet$ and $\mathrm{CO}$, it is possible for a bicyclic radical (4) to be formed or for the ring to open and then form a ketenyl radical species (6). As expected, the formation of 4 is more favorable and has an activation barrier that is $\sim 19 \mathrm{kcal} / \mathrm{mol}(298 \mathrm{~K})$ lower than the activation barrier to form 6 . Furthermore, the bicyclic intermediate 4 is $\sim 14.1 \mathrm{kcal} / \mathrm{mol}$ more stable than 6 at $298 \mathrm{~K}$. The next step is the formation of 5 . This either occurs through the cyclopropyl ring opening of 4 or via a ring closure of 6 . The activation barrier for the most favorable pathway ( $4 \rightarrow 5$ ) is $7.4 \mathrm{kcal} / \mathrm{mol}(298 \mathrm{~K})$. For comparison to previous results, our stepwise bottom-of-the-well energies with scaled ZPE corrections at the B3LYP/6$311+\mathrm{G}^{* *} / / \mathrm{B} 3 \mathrm{~L}$ YP/6-31G* level are similar to the values calculated by Morokuma et al. ${ }^{12}$ at the G2M(rcc,MP2) level for similar structures, such as TS 3-4 and TS 4-5. Our activation barriers are 50.5 and $55.3 \mathrm{kcal} / \mathrm{mol}$, which compare to calculated G2M(rcc,MP2) values of 48.9 and 51.9 $\mathrm{kcal} / \mathrm{mol},{ }^{12}$ respectively. The final step is the simple decomposition of 5 to yield $\mathrm{Cp} \bullet$ and $\mathrm{CO}$ as products.

Overall, the activation barrier in the initial step of $3 \rightarrow 4$ is calculated to be $\sim 51 \mathrm{kcal} / \mathrm{mol}$ 
at all temperatures evaluated. This is similar to the experimentally determined activation energy of $44.0 \pm 0.9 \mathrm{kcal} / \mathrm{mol}$ at 1000 to $1580 \mathrm{~K}^{36}$ Using simple transition state theory ${ }^{37}$ for the calculated free energies of activation along the lowest energy pathway, $3 \rightarrow 4 \rightarrow 5$, the overall reaction rate for the decomposition of phenoxy radical to $\mathrm{Cp} \bullet$ and $\mathrm{CO}$ is $20 \mathrm{~s}^{-1}$ at $1000 \mathrm{~K}$, which compares to experimentally determined values of $10 \pm 5 \mathrm{~s}^{-1}(1000 \mathrm{~K})^{3}$ and $63.4 \mathrm{~s}^{-1} \cdot 36,38$

At all temperatures studied here, the reaction pathway which proceeds through the nonplanar bicyclic radical (4) is lower in energy. However, it is interesting to note that the energy gap between the two pathways steadily decreases with increasing temperature, and at temperatures greater than $\sim 2200 \mathrm{~K}$, the ring opening pathway yielding the ketenyl radical intermediate (6) will be the more favorable.

Path II - Dioxiranyl Radical Intermediate. The dioxiranyl radical (8) is formed by a ring closure (1,1-addition) of the terminal oxygen of phenylperoxy radical (1) onto the phenyl ring. The activation barrier for this rearrangement $(1 \rightarrow \mathbf{8})$ is $27.2 \mathrm{kcal} / \mathrm{mol}$ at $298 \mathrm{~K}$, which is very similar $(\sim 0.1 \mathrm{kcal} / \mathrm{mol})$ to the relative free energy of formation of phenoxy radical and an oxygen atom $(1 \rightarrow 3)$. At temperatures $\geq 430 \mathrm{~K}$, the overall free energy for the $1 \rightarrow 3$ pathway attains greater stability than the initial step of the dioxiranyl radical pathway.

Subsequent decomposition of the dioxiranyl radical (8) can possibly occur along two distinct pathways. The first pathway, proposed originally by Carpenter, ${ }^{7}$ involves rearrangement to form a 7-membered ring intermediate (10). An alternate pathway, occurs via cyclization of 8 to form 15 in a step that is analogous to $3 \rightarrow 4$ in the phenoxy radical decomposition.

The first step along Carpenter's proposed pathway is the isomerization of $\mathbf{8}$ to yield the 7membered ring 10. Carpenter calculated, using the PM3 method, that the $\Delta \mathrm{H}^{\ddagger}$ for this reaction is $10.5 \mathrm{kcal} / \mathrm{mol}(298 \mathrm{~K})$ with an overall exothermicity of $-74.5 \mathrm{kcal} / \mathrm{mol}$. Correlation between these results and those obtained at the B3LYP level are good, but require the inclusion of the intermediate 9 in the reaction step. With this addition, the B3LYP method calculates the overall 
activation barrier to this isomerization to be $11.3 \mathrm{kcal} / \mathrm{mol}(298 \mathrm{~K})$ with an overall exoergicity (8 $\rightarrow 9 \rightarrow 10$ ) of $-70.7 \mathrm{kcal} / \mathrm{mol}$. A direct transition state to form 10 from 8 also exists, but the activation barrier is very high $(70.0 \mathrm{kcal} / \mathrm{mol})$.

The stepwise isomerization $(8 \rightarrow 9 \rightarrow 10)$ begins with the scission of the O-O bond of 8 . This reaction has an activation barrier of $11.3 \mathrm{kcal} / \mathrm{mol}(298 \mathrm{~K})$ and results in the intermediate 9. Structure 9 can exist as a doublet or quartet as shown by Mebel and Lin, ${ }^{13}$ and the doublet state should be more stable. The energy for this intermediate, as calculated with the B3LYP method, is greater than the energy of the transition state leading to its formation from 8 or the energy of the transition state proceeding from 9 to 10 . This is a result of excessive spin contamination in 9 , but the transition states (TS 8-9 and TS 9-10) do not suffer from this problem. The activation barrier for $\mathbf{9} \rightarrow \mathbf{1 0}$ is lower in Gibbs free energy at all temperatures studied than the activation barrier for the $\mathrm{O}-\mathrm{O}$ bond scission. Therefore, the scission of the $\mathrm{O}-\mathrm{O}$ bond is the greatest barrier (11.3 kcal/mol) for the isomerization of 8 to yield the 7-membered ring, 10.

Structure 10 can then isomerize to form the nonplanar bicyclic radical (11), the pyranyl species (17), or the acyclic structure (21). The lowest activation barrier for the rearrangement of 10 at $298 \mathrm{~K}, 23.4 \mathrm{kcal} / \mathrm{mol}$, leads to the formation of 21 , followed by the isomerization to 11 ( $39.5 \mathrm{kcal} / \mathrm{mol})$ and then the production of 17 with a barrier of $53.8 \mathrm{kcal} / \mathrm{mol}$. These values are similar to the enthalpic barriers that Carpenter ${ }^{7}$ calculated of 30.0 and $37.0 \mathrm{kcal} / \mathrm{mol}$, leading to the first two intermediates (21 and 11, respectively). Carbon dioxide is readily extruded from 11 with an activation barrier of $8.7 \mathrm{kcal} / \mathrm{mol}$ via the $11 \rightarrow 12 \rightarrow 14$ pathway to yield $\mathrm{Cp} \bullet$ and $\mathrm{CO}_{2}$. The simple decomposition of 17 to the pyranyl radical (18) and $\mathrm{CO}$ is essentially barrierless at all temperatures. (Please note that the relative free energies for $12 \rightarrow 14$ in this section differ from our earlier results on the dioxiranyl pathway in phenylperoxy radical and is due to a previously undetected spreadsheet error for $\mathrm{CO}_{2}$ in Figure 3 of reference 8 . The exoergicity for $12 \rightarrow 14$ is $-45.3 \mathrm{kcal} / \mathrm{mol}$.)

An alternate pathway in the unimolecular decomposition of the dioxiranyl radical is via a mechanism similar to the phenoxy radical decomposition to generate $\mathrm{Cp} \mathrm{p}^{\bullet}$ and $\mathrm{CO}$. The 
formation of a nonplanar tricyclic radical (15) is rather endoergic, but surprisingly not as much as for the formation of the bicyclic radical (4) along the phenoxy radical pathway. At $298 \mathrm{~K}$, the activation barrier is $46.7 \mathrm{kcal} / \mathrm{mol}$ to create 15 . Simple decomposition of 15 to $\mathrm{Cp}$ and $\mathrm{CO}_{2}$ will occur readily with an activation barrier of only $\sim 6.8 \mathrm{kcal} / \mathrm{mol}$, resulting in an overall exoergic reaction of $-138.2 \mathrm{kcal} / \mathrm{mol}(298 \mathrm{~K})$ from 15 .

Ring opening of $\mathbf{8}$ to generate $\mathbf{1 6}$ as the product is expected to be a higher energy pathway. Structure 16 is higher in energy by $\sim 15 \mathrm{kcal} / \mathrm{mol}$ than the transition state to form 15; however, we have not searched for the corresponding transition state to form 16. But once again, it appears from the energetic trends that opening of the ring will be favored over the formation of the tricyclic radical intermediate (15) at high temperatures.

At all temperatures evaluated it appears that the energetically most favored pathway for the decomposition of the dioxiranyl radical (8) is the one that is analogous to the Carpenter pathway, ie. $8 \rightarrow 9 \rightarrow 10 \rightarrow(14,18$ or 23$)$.

Path III - 1,2-Dioxetanyl Radical Intermediate. The formation of a 1,2-dioxetanyl intermediate (19) is endoergic by $+44.8 \mathrm{kcal} / \mathrm{mol}$ with a free energy of activation of 46.2 $\mathrm{kcal} / \mathrm{mol}$ at $298 \mathrm{~K}$. Both the activation barrier and ground state free energy of 19 increase gradually with increasing temperature, and at $1250 \mathrm{~K}$, the energies are 50.2 and $+46.3 \mathrm{kcal} / \mathrm{mol}$, respectively. The isomerization of 19 to form the $o$-phenylquinone radical (20) is essentially barrierless at temperatures $<500 \mathrm{~K}$ and remains below $2 \mathrm{kcal} / \mathrm{mol}$ at temperatures above $1250 \mathrm{~K}$. The isomerization of 20 can either proceed through an epoxide-like transition state (TS 20-10) to yield the 7-membered ring (10) or through a scission of the $C_{1}-C_{2}$ bond, producing the acyclic structure 21. Contrary to the phenoxy and dioxiranyl radical pathways, the ring-opening mechanism is the lowest energy pathway for the unimolecular rearrangement of 20 , and the energy gap between the stability of the two transition states (TS 20-10 and TS 20-21) only increases with temperature. If 10 or 21 are produced, they can decompose in the same manner as described in path $\mathrm{II}$, resulting in $\mathrm{Cp}$. and $\mathrm{CO}_{2}$, pyranyl radical (18) and $\mathrm{CO}$, or the acyclic 
ketenyl radical structure (23). The ring-opening pathway appears to be the optimum pathway at all temperatures for the decomposition of phenylperoxy radical if it proceeds through a 1,2dioxetanyl intermediate.

Path IV - 1,3-Peroxy Radical Intermediate. The isomerization of phenylperoxy radical (1) to form a 1,3-peroxy radical (24) requires a tremendous amount of energy. Not only does this intermediate lose aromaticity, but it also results in a bicyclic, anti-Bredt ${ }^{39}$ alkene that cannot stabilize the radical. The energy barrier for the production of 24 at $298 \mathrm{~K}$ exceeds 102 $\mathrm{kcal} / \mathrm{mol}$ and only increases with increasing temperature. Thus, if phenylperoxy radical is able to gain enough internal energy to isomerize to structure 24, the final products of pyranyl radical (18) and $\mathrm{CO}$ are much lower in energy.

The first step is the formation of an epoxide species (25) which can then rearrange to form 26, which is an isomer of the stable 7-membered ring intermediate (10) along the dioxiranyl radical pathway. The two structures ( 25 and 26$)$ are very similar in ground state energies at $298 \mathrm{~K}$, but as the temperature increases, intermediate $\mathbf{2 6}$ gains moderately in stability as compared to 25 . Structure 26 can then close the ring to form a nonplanar bicyclic radical (27), which is slightly more stable than the phenylperoxy radical at all temperatures. The activation energy for this process is $32.8 \mathrm{kcal} / \mathrm{mol}(298 \mathrm{~K})$ and is the highest barrier along this pathway after the costly initial step to form 24 . Structure 27 can then lose $\mathrm{CO}$ to yield the final pyranyl radical (18) product.

Path V-p-Phenylquinone Radical Intermediate. Another pathway that was explored, but is highly improbable, is the transformation of the phenylperoxy radical to $p$-phenylquinone radical (28) via a 1,4-addition. The free energy of activation for this reaction is $91.4 \mathrm{kcal} / \mathrm{mol}$ at $298 \mathrm{~K}$ and steadily rises to $94.0 \mathrm{kcal} / \mathrm{mol}$ at $1250 \mathrm{~K}$; however, the overall reaction is exoergic by -17.9 to $-19.1 \mathrm{kcal} / \mathrm{mol}$ throughout the same temperature range. The pathway explored from this point was chosen to provide an alternate mechanism for the production of $\mathrm{Cp} \bullet$. 
The $p$-phenylquinone radical (28) incurs a significant energetic penalty to form the bicyclic radical structure (29), which can then break open the ring to form the stable intermediate $\mathbf{3 0}$.

Structure 30 can than rearrange in two different ways. The lowest energy pathway yields acetylene and a g-lactone radical (31). The other possibility is an isomerization that results in another bicyclic radical (32) which leads to $\mathrm{CO}_{2}$ extrusion and the formation of $\mathrm{Cp}^{*}$. The key transition states at the diverging point are TS 30-31 and TS 30-32. As the temperature increases, the stability of TS 30-31 is favored, thereby increasing the relative activation barrier gap further promoting the production of $\mathbf{3 1}$.

Comparison of theoretical methods. In order to evaluate the quantitative accuracy of the B3LYP energies, we have also calculated single point energies at the $\operatorname{CASSCF}(7,8) / 6-31 \mathrm{G}^{*}$, CAS-MP2(7,8)/6-31G* and UCCSD(T)/6-31G** levels for some key intermediates and transition states. These relative energies (at the bottom of the well) are provided in Table 3.2.2. The CASSCF calculations are problematic due to the choice of the active space for these diverse structures. As seen in Table 3.2.2, there is a lack of convergence between the CASSCF energies and the other methods, and is most likely due to the choice of the active space. A correct treatment of this system by CASSCF methods will require a more extensive analysis.

However, for comparison to the B3LYP energies, we have also calculated UCCSD(T)/6$31 \mathrm{G}^{* *}$ single point energies and these are also shown in Table 3.2.2. The UCCSD(T) energies are in good qualitative and quantitative agreement with the B3LYP results, except for structures 9 and TS 9-10, which have spin contamination and/or UCCSD(T) convergence problems.

Comparison of Reaction Pathways at Different Temperatures. In comparing the lowest energy mechanism for each pathway that leads to $\mathrm{Cp} \bullet$ production, the 1,1-dioxiranyl radical pathway appears to be the most favored pathway from 298 to $1250 \mathrm{~K}$ based on the lowest overall activation barrier, TS 8-9. When considering the largest cumulative activation barrier, the 1,2-dioxetanyl radical pathway (via TS 1-19) is even more favorable than 
Table 3.2.2. Relative energies $(\mathrm{kcal} / \mathrm{mol})$ at different theoretical levels for a few important intermediates and transition states. ${ }^{\mathrm{a}}$

\begin{tabular}{|c|c|c|c|c|c|c|c|c|c|}
\hline & 1 & TS $1-8$ & 8 & TS $8-9$ & 9 & TS $9-10$ & 10 & TS $10-11$ & 11 \\
\hline$\overline{\operatorname{CASSCF}(7,8) / 6-31 \mathrm{G}^{*}}$ & 0.0 & $\overline{\overline{17.1}}$ & $4 \overline{4.7}$ & 9.0 & $4 \overline{45.4}$ & 26.5 & -36.9 & $-16 . \overline{7}$ & -59.7 \\
\hline CAS-MP2 $(7,8) / 6-31 G^{\star}$ & 0.0 & 36.9 & 31.7 & 40.5 & 61.5 & 53.0 & -63.8 & -8.6 & -57.9 \\
\hline UMP2/6-31G & 0.0 & 47.5 & 28.3 & 50.7 & 67.2 & 55.5 & -32.9 & 2.5 & -44.1 \\
\hline UMP3/6-31G** & 0.0 & 40.5 & 25.2 & 51.4 & 47.1 & 59.5 & -37.5 & 0.2 & -43.5 \\
\hline UMP4(SDQ)/6-31G** & 0.0 & 36.6 & 21.4 & 43.6 & 45.5 & 52.0 & -42.0 & -4.9 & -47.3 \\
\hline UCCSD/6-31G** & 0.0 & 29.0 & 18.3 & 39.5 & $40.0^{b}$ & $54.1^{\mathrm{b}}$ & -45.8 & -9.2 & -45.9 \\
\hline $\operatorname{UCCSD}(T) / 6-31 G^{\star \star}$ & 0.0 & 28.4 & 19.1 & 34.6 & $\ldots$ & $\ldots b$ & -43.6 & -8.6 & -43.6 \\
\hline$\overline{U B 3 L Y P / 6-31 G^{*}}$ & 0.0 & 28.4 & 22.8 & $39 . \overline{8}$ & 46.2 & 37.1 & -45.9 & -4.9 & -36.7 \\
\hline UB3LYP/6-311+G* & 0.0 & 28.6 & 23.6 & 36.1 & 43.4 & 32.6 & -47.6 & -7.3 & -38.3 \\
\hline$\overline{U B 3 L Y P / 6-311+G^{\star \star}\left\langle S^{2}\right\rangle}$ & 0.76 & 0.79 & 0.79 & 0.76 & 1.82 & 0.76 & 0.78 & 0.80 & 0.78 \\
\hline $\operatorname{UCCSD}(T) / 6-31 G^{* *}\left\langle S^{2}\right\rangle$ & 0.77 & 1.42 & 1.21 & 1.20 & 2.24 & 0.76 & 1.27 & 1.36 & 0.97 \\
\hline
\end{tabular}

The B3LYP/6-31 G* geometry was used in each case.

'The CCSD equations could not be converged to the necessary accuracy $\left(<10^{-7}\right.$ au) by Gaussian 98 in order to complete the UCCSD(T) calculation. The corresponding CCSD energies for 9 ad TS 9-10 reflect a $10^{-5}$ au convergence.

the phenoxy radical pathway (via TS 4-5) from 298 to $750 \mathrm{~K}$, but is significantly less available than the 1,1-dioxiranyl radical pathway. As the temperature increases to $1000 \mathrm{~K}$, the phenoxy radical decomposition pathway's overall activation barrier decreases to where it is roughly equivalent to the 1,2-dioxetanyl radical pathway, and by $1250 \mathrm{~K}$, the phenoxy radical pathway (Path I) is becoming competitive with the 1,1-dioxiranyl radical pathway as the barriers along Path I become more accessible.

This comparison neglects that the lowest energy route along the 1,1-dioxiranyl pathway (via $8 \rightarrow 9 \rightarrow 10 \rightarrow 21 \rightarrow 22 \rightarrow 23$ ) yields the acyclic species, 23. Therefore, decomposition of the phenylperoxy radical $(1)$ at low to intermediate temperatures $(<1250 \mathrm{~K})$ should produce greater amounts of products from a ring-opened structure than the production of $\mathrm{Cp}^{\bullet}$. Such ringopened products (or fragments thereof) have been seen experimentally. ${ }^{35}$ 
The reaction mechanisms have been compared at varying temperatures which are relevant to atmospheric or combustion processes. This potential energy surface will serve as a model for further research of oxidation pathways for heteroaromatic radicals that are relevant to coal. Further study in determining the overall reaction rates for each pathway is needed to fully confirm these conclusions.

\section{References for Section 3.2}

(1) Brezinsky, K. Prog. Energy Combust. Sci. 1986, 12, 1-24.

(2) Bittker, D. A. Combust. Sci. and Tech. 1991, 79, 49-72.

(3) Colussi, A. J.; Zabel, F.; Benson, S. W. Int. J. Chem. Kinet. 1977, 9, 161-178.

(4) Zhang, H.-Y.; McKinnon, J. T. Combust. Sci. and Tech. 1995, 107, 261-300.

(5) Benson, S. W. J. Am. Chem. Soc. 1965, 87, 972.

(6) Yu, T.; Lin, M. C. J. Am. Chem. Soc. 1994, 116, 9571-9576.

(7) Carpenter, B. K. J. Am. Chem. Soc. 1993, 113, 9806-9807.

(8) Barckholtz, C.; Fadden, M. J.; Hadad, C. M. J. Phys. Chem. A 1999, 103, 8108-8117.

(9) Carpenter, B. K. J. Phys. Chem. 1995, 99, 9801-9810.

(10) Mebel, A. M.; Diau, E.W. G.; Lin, M. C.; Morokuma, K. J. Am. Chem. Soc. 1996, 118, 9759-9771.

(11) Olivella, S.; Sole, A.; Garcia-Raso, A. J. Phys. Chem. 1995, 99, 10549.

(12) Liu, R.; Morokuma, K.; Mebel, A. M.; Lin, M. C. J. Phys. Chem. 1996, 100, 9314-9322.

(13) Mebel, A. M.; Lin, M. C. J. Am. Chem. Soc. 1994, 116, 9577-9584.

(14) Lin, M. C.; Mebel, A. M. J. Phys. Org. Chem. 1995, 8, 407-420.

(15) Mebel, A. M.; Lin, M. C.; Yu, T.; Morokuma, K. J. Phys. Chem. A 1997, 101, 31893196.

(16) Barckholtz, C.; Barckholtz, T. A.; Hadad, C. M. J. Am. Chem. Soc. 1999, 121, 491-500.

(17) Frisch, M. J.; Trucks, G. W.; Schlegel, H. B.; Gill, P. M. W.; Johnson, B. G.; Robb, M. A.; Cheeseman, J. R.; Keith, T.; Petersson, G. A.; Montgomery, J. A.; Raghavachari, K.; Al-Laham, M. A.; Zakrzewski, V. G.; Ortiz, J. V.; Foresman, J. B.; Peng, C. Y.; Ayala, P. Y.; Chen, W.; Wong, M. W.; Andres, J. L.; Replogle, E. S.; Gomperts, R.; Martin, R. L.; Fox, D. J.; Binkley, J. S.; Defrees, D. J.; Baker, J.; Stewart, J. J. P.; Head-Gordon, M.; Gonzalez, C.; Pople, J. A. Gaussian 94, Revision D.3, Gaussian, Inc.; Pittsburgh, PA, 1995.

(18) Frisch, M. J.; Trucks, G. W.; Schlegel, H. B.; Scuseria, G. E.; Robb, M. A.; Cheeseman, J. R.; Zakrzewski, V. G.; Montgomery, J. A., Jr.; Stratmann, R. E.; Burant, J. C.; Dapprich, S.; Millam, J. M.; Daniels, A. D.; Kudin, K. N.; Strain, M. C.; Farkas, O.; Tomasi, J.; Barone, V.; Cossi, M.; Cammi, R.; Mennucci, B.; Pomelli, C.; Adamo, C.; Clifford, S.; Ochterski, J.; Petersson, G. A.; Ayala, P. Y.; Cui, Q.; Morokuma, K.; Malick, D. K.; Rabuck, A. D.; Raghavachari, K.; Foresman, J. B.; Cioslowski, J.; Ortiz, J. V.; Stefanov, B. B.; Liu, G.; Liashenko, A.; Piskorz, P.; Komaromi, I.; Gomperts, R.; Martin, R. L.; Fox, D. J.; Keith, T.; Al-Laham, M. A.; Peng, C. Y.; Nanayakkara, A.; Gonzalez, C.; Challacombe, M.; Gill, P. M. W.; Johnson, B.; Chen, W.; Wong, M. W.; 
Andres, J. L.; Gonzalez, C.; Head-Gordon, M.; Replogle, E. S.; Pople, J. A. Gaussian 98, Revision A.6, Gaussian, Inc.; Pittsburgh, PA, 1998.

(19) Becke, A. D. Phys. Rev. A 1988, 38, 3098-3100.

(20) Lee, C.; Yang, W.; Parr, R. G. Phys. Rev. B. 1988, 37, 785-789.

(21) Becke, A. D. J. Chem. Phys. 1993, 98, 1372.

(22) Hehre, W. J.; Radom, L.; Schleyer, P. v. R.; Pople, J. A. Ab Initio Molecular Orbital Theory; John Wiley \& Sons: New York, 1986

(23) Bauschlicher, C. W., Jr.; Langhoff, S. R. Mol. Phys. 1999, 96, 471.

(24) Hegarty, D.; Robb, M. A. Mol. Phys. 1979, 38, 1795.

(25) Eade, R. H.; Robb, M. A. Chem. Phys. Lett. 1981, 83, 362.

(26) Schlegel, H. B.; Robb, M. A. Chem. Phys. Lett. 1982, 93, 43.

(27) Yamamoto, N.; Vreven, T.; Robb, M. A.; Frisch, M. J.; Schlegel, H. B. Chem. Phys. Lett. 1996, 250, 373.

(28) Stanton, J. F.; Gauss, J.; Watts, J. D.; Lauderdale, W. J.; Bartlett, R. J. Int. J. Quantum Chem. 1992, $S 26,879$.

(29) Gonzalez, C.; Schlegel, H. B. J. Chem. Phys. 1989, 90, 2154.

(30) Gonzalez, C.; Schlegel, H. B. J. Phys. Chem. 1990, 94, 5523.

(31) Rablen, P. R., Yale University, 1994.

(32) Scott, A. P.; Radom, L. J. Phys. Chem. 1996, 100, 16502-16513.

(33) Chase, M. W., Jr. NIST-JANAF Thermochemical Tables; Washington, DC: American Chemical Society; New York: American Institute of Physics for the National Institute of Standards and Technology, 1998.

(34) Knyazev, V. D.; Slagle, I. R. J. Phys. Chem. 1995, 99, 2247-2249.

(35) Chai, Y.; Pfefferle, L. D. Fuel 1998, 77, 313-320.

(36) Lin, C-Y.; Lin, M. C. J. Phys. Chem. 1986, 90, 425-431.

(37) The calculated kinetic treatment was performed as discussed in reference 3.

(38) The expression listed in the paper (ref. 28) is $10^{11.90 \pm 0.20} \exp \left(-23900 \pm 450 / \mathrm{T}\right.$ ) $\mathrm{s}^{-1}$, and corresponds to a range of 25.5 to $157.6 \mathrm{~s}^{-1}$, based on the published error bars.

(39) Bredt, J.; Thouet, H.; Schmitz, J. Liebigs Ann. Chem. 1924, 437, 1. 


\subsection{The Rearrangement Pathways of Arylperoxy Radicals. I. The Azabenzenes}

\subsubsection{Executive Summary}

The potential energy surfaces for the reaction of pyridinyl radicals with $\mathrm{O}_{2}$ have been studied using the B3LYP method. The initial production of the pyridinylperoxy radical followed by either simple decomposition or rearrangement to yield the intermediates (pyridinyloxy, dioxiranylpyridinyl or dioxetanylpyridinyl radicals) have been explored. Transition state structures for most of the steps are presented as well as relative free energies over a range of temperatures from 298 to $2000 \mathrm{~K}$. The energetics of the analogous intermediates for the reaction of $\mathrm{O}_{2}$ and other azabenzene radicals derived from pyridazine, pyrimidine, and pyrazine are also provided. $\mathrm{O}_{2}$ dissociation from the arylperoxy radical is preferred rather than the loss of $\mathrm{O}$ atom to generate the corresponding aryloxy radical, and this preference is contrary to phenylperoxy radical decomposition. However, the formation of a dioxiranyl radical intermediate is the most accessible intermediate from the peroxy precursor at temperatures $\leq 500 \mathrm{~K}$. Dioxetanyl intermediates are less favored but may provide a route to $\mathrm{NO}_{\mathbf{x}}$ generation from nitrogen substitution in aromatic fuels.

\subsubsection{Introduction}

Understanding the processes involved in coal combustion is difficult due to the complex nature of coal and the diverse structural units present in the different ranks of coal. ${ }^{1}$ In order to resolve the chemical processes that occur during coal combustion, many workers have relied on the use of monocyclic model compounds, such as benzene, pyridine, furan, and thiophene, in order to gain some understanding into the combustion of organic fuels. As the "standard" aromatic ring, numerous studies have been completed on the oxidative, thermal decomposition of benzene. $2,3,4,5,6$

Nitrogen-containing aromatic rings also constitute a significant fraction of coal, and oxidation of these units contribute to $\mathrm{NO}_{\mathrm{x}}$ generation in coal-fired boilers. ${ }^{7}$ Most studies with 
pyridine or the diazines have focused on the pyrolysis ${ }^{8,9,10}$ of these compounds, and oxidative pyrolysis has been less well studied. ${ }^{11} \mathrm{C}-\mathrm{H}$ bond dissociation enthalpies (BDEs) have been determined for some of these monocyclic azabenzenes, and a significant stabilizing effect on the radical has been noted when nitrogen is present in the ring. ${ }^{12}$

Several studies on the reaction of phenyl radical (1) with $\mathrm{O}_{2}$ have shown the importance of phenylperoxy radical (2) as a key intermediate. ${ }^{13,14}$ As depicted in Figure 3.3.1, the phenylperoxy radical can then further decompose or rearrange via a number of different pathways, including a phenoxy radical (3), a dioxiranyl radical (4), or possibly a dioxetanyl radical (5) before ultimately decomposing to the cyclopentadienyl radical, $\mathrm{CO}, \mathrm{CO}_{2}$ and a number of other hydrocarbons. ${ }^{6,15,16}$

Recently, we have explored the mechanisms for the reaction of phenyl radical with $\mathrm{O}_{2}$ in great detail (including transition states), and earlier, we have presented a thermodynamic study for the decomposition of some other aryl species, including the 2-pyridinyl radical. ${ }^{15} \mathrm{We}$, and others, have shown that density functional theory (DFT) $\operatorname{methods}{ }^{17}$ can be accurately applied to aromatic radicals, and at reasonable expense, as spin contamination is not a significant problem. ${ }^{12,18}$

In this paper, we present a detailed potential energy surface for the reaction of pyridinyl radicals with $\mathrm{O}_{2}$, as we have explored the analogous decomposition pathways as depicted in Figure 3.3.1 for phenyl radical with $\mathrm{O}_{2}$. In particular, the reactivity of the pyridinylperoxy and diaza-arylperoxy radicals are compared with phenylperoxy radicals at temperatures ranging from 298 to $2000 \mathrm{~K}$.

\subsubsection{Computational methods}

All geometry optimizations, vibrational frequency calculations, and single point energy determinations were completed with Gaussian 94 and 98 at the Ohio Supercomputer Center or on our IBM RS/6000 workstations. ${ }^{19,20}$ 
<smiles>OOc1ccccc1</smiles>

2b

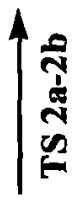

\section{సิ}

TS 1-2a

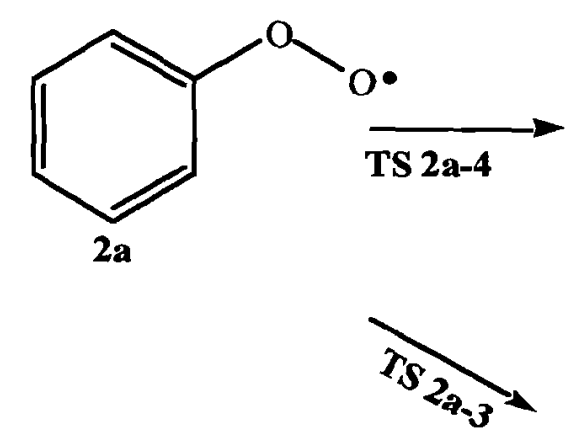

TS 2b-5b
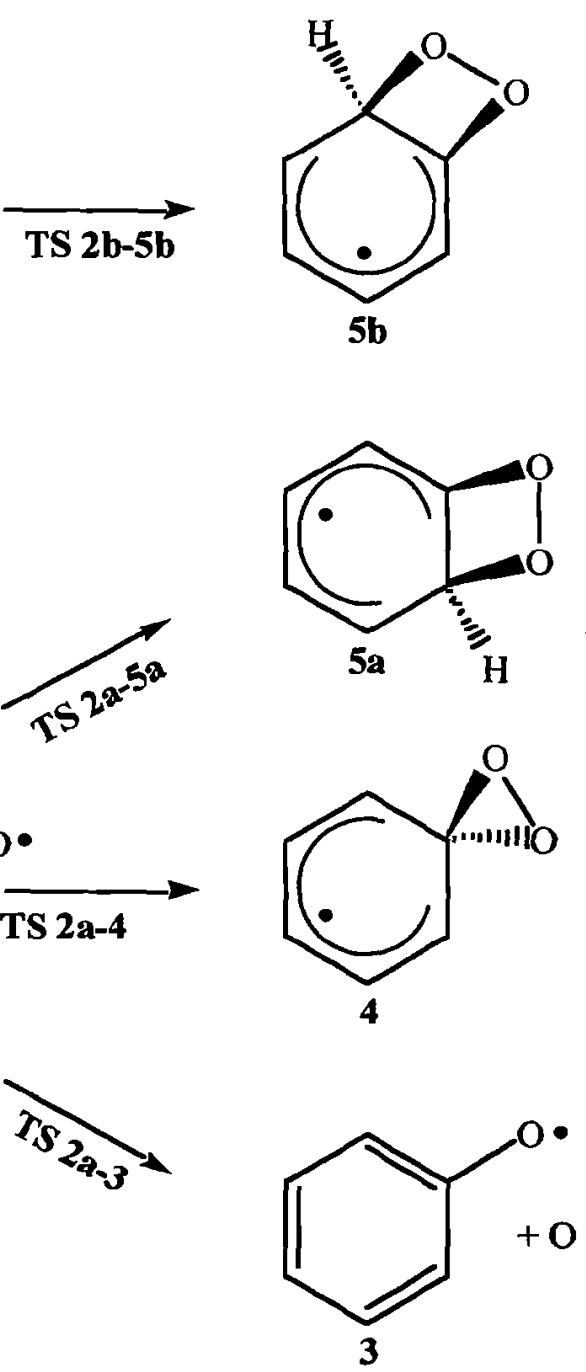

Figure 3.3.1. Reaction mechanism of phenyl radical with $\mathrm{O}_{2}$. This numbering sequence will be used throughout the text, tables and figures to identify the analogous intermediate and transition state for each azabenzene system.

The B3LYP/6-31G* hybrid density functional theory level was employed to determine the optimized geometries and vibrational frequencies of all stationary points. ${ }^{21,22,23,24}$ Single point energies were also determined at the B3LYP/6-311+G** level, as it has been shown that there is a small basis set effect with oxygen-containing systems when determining $\mathrm{C}-\mathrm{H}$ bond dissociation energies. ${ }^{25}$ The B3LYP/6-311+G** single point energies should provide better relative energies. We have also determined single point energies at the $\mathrm{UCCSD}(\mathrm{T}) / 6-31 \mathrm{G} * *$ 
level of theory for several key intermediates and transition states in order to compare with our B3LYP results. ${ }^{26}$. All basis sets used 6 cartesian $d$ functions.

Vibrational frequencies were calculated for each stationary point in order to confirm each structure as a minimum or a saddle point, and each stationary point had the correct number of real and imaginary vibrational frequencies. The zero-point vibrational energy (ZPE) corrections were also obtained and scaled by a factor of $0.9806 .{ }^{27}$ All transition states were confirmed to connect to the corresponding reactant and product by displacing (typically $10 \%$ ) the geometry along the reaction coordinate for the imaginary vibrational frequency in either direction, which was then followed by a careful optimization (opt $=$ calcfc or opt $=$ calcall) to the corresponding minimum.

Spin contamination for most stationary points was negligible. The $\left\langle S^{2}\right\rangle$ values for all of the minima and for most of the transition states fell below 0.80 for these doublet species. Those specific transition states that suffered from excessive spin contamination will be discussed below, and their energies and geometries are considered to be suspect.

To determine the thermodynamic contribution to the free energy of each molecule at temperatures ranging from 298 to $2000 \mathrm{~K}$, Thermo $94^{28}$ was used to calculate the partition function contributions derived from the optimized geometry and the unscaled vibrational frequencies as calculated by Gaussian. The overall Gibbs free energy at each temperature was derived from the single point energy, the scaled ZPE, the thermodynamic contribution (enthalpy and entropy), and the electronic contribution to the free energy. The only molecule which received different treatment was $\mathrm{O}$ atom, where experimentally ${ }^{29}$ determined splitting energies were included in the electronic component of the partition function for the free energy calculation.

The energies discussed throughout are Gibbs free energies computed at the B3LYP/6$311+\mathrm{G}^{* *} / / \mathrm{B} 3 \mathrm{LYP} / 6-31 \mathrm{G}^{*}$ level (at 298K, unless noted otherwise), and are relative to the Gibbs free energy of the corresponding arylperoxy radical. 


\subsubsection{Mechanism of the pyridinyl radical $+\mathrm{O}_{2}$ reaction}

\subsubsection{Formation of the pyridinylperoxy radical intermediate $(1 \rightarrow 2 a)$}

Experimentally, pyridinylperoxy radicals are formed by the rapid addition of $\mathrm{O}_{2}$ to the pyridinyl radical in the condensed phase. ${ }^{30}$ At $298 \mathrm{~K}$, this reaction is calculated to be exoergic by $-28.5 \mathrm{kcal} / \mathrm{mol}$ for 2-pyridinyl radical, $-30.4 \mathrm{kcal} / \mathrm{mol}$ for 3-pyridinyl radical, and $-29.9 \mathrm{kcal} / \mathrm{mol}$ for 4-pyridinyl radical. The free energy of activation $\left(\Delta \mathrm{G}^{*}, 298 \mathrm{~K}\right)$ for the formation of 2 pyridinylperoxy and 4-pyridinylperoxy radicals from their respective precursors is $6.2 \mathrm{kcal} / \mathrm{mol}$, while the corresponding value for 3-pyridinylperoxy radical is $7.2 \mathrm{kcal} / \mathrm{mol}$.

The transition states for the formation of the pyridinylperoxy radicals have been found, but they suffer from excessive spin contamination $\left(\left\langle S^{2}\right\rangle \sim 1.8\right)$ and have very small imaginary vibrational frequencies, ranging from $7.9 i$ to $16.5 i \mathrm{~cm}^{-1}$. These values are very similar to previously reported calculations for the addition of $\mathrm{O}_{2}$ to phenyl radical where $\Delta \mathrm{G}=-32.3$ $\mathrm{kcal} / \mathrm{mol}$ and $\Delta \mathrm{G}^{*}=6.1 \mathrm{kcal} / \mathrm{mol}$, and the transition state for formation of phenylperoxy radical had $\left\langle S^{2}\right\rangle=1.76$ and an imaginary vibrational frequency of $6.2 i \mathrm{~cm}^{-1} .{ }^{16}$ The lowest energy conformations of the 2-, 3-, and 4-pyridinylperoxy radicals have $C_{s}$ symmetry with ${ }^{2} \mathrm{~A}$ " electronic ground states.

The rearrangement or unimolecular decomposition of the pyridinylperoxy radicals to yield several different intermediates was explored. In order to compare structures between phenyl radical and the aza-aryl radicals, we have chosen to use a consistent naming system, as depicted in Figure 3.3.1, where for example, each 2a isomer is a peroxy radical derived from the corresponding aryl radical. Also, the $\mathbf{a}$ isomer is always more stable than the $\mathbf{b}$ isomer when there is more than one unique way to orient the group. (For phenyl radical, these $\mathbf{a}$ and $\mathbf{b}$ isomers are the same structure.)

The first pathway in the unimolecular decomposition of pyridinylperoxy radical (2a) is the cleavage of the $\mathrm{O}-\mathrm{O}$ bond to yield the pyridinyloxy radical and $\mathrm{O}$ atom $(\mathbf{2 a} \rightarrow 3)$. Another pathway would be the rearrangement of the pyridinylperoxy radical to form a dioxiranyl intermediate $(2 a \rightarrow 4)$. Finally, the production of dioxetanyl intermediates from either the 
lowest energy conformation of pyridinylperoxy radical $(2 \mathbf{a} \rightarrow \mathbf{5 a})$ or from a rotation of the peroxy functional group followed by ring closure $(\mathbf{2 a} \rightarrow \mathbf{2 b} \rightarrow \mathbf{5 b}$ ) are also possibilities. All of the energies reported in the tables as well as those discussed within the text are relative to the energy of the most stable conformation of the respective arylperoxy radical (ie. the corresponding 2a structure).

\subsubsection{Unimolecular decomposition of the pyridinylperoxy radicals to yield pyridinyloxy radicals and $\mathrm{O}$ atom $(2 \mathrm{a} \rightarrow 3)$}

Pyridinylperoxy radicals can decompose via the homolysis of the $\mathrm{O}-\mathrm{O}$ bond to produce the pyridinyloxy radical (3, Figure 3.3.2) and $\mathrm{O}$ atom. This reaction is an endoergic process at $298 \mathrm{~K}$ for the 2-, 3- and 4-pyridinylperoxy radicals. The order of stability of the pyridinyloxy radicals as compared to their peroxy radical precursors is 3->2->4-pyridinyloxy radical. From the B3LYP/6-31G* optimized geometries of these molecules (Figure 3.3.3), it is apparent that all of the pyridinyloxy radicals delocalize the unpaired electron around the ring, resulting in some enhanced stability. This delocalization also occurs for phenoxy radical. ${ }^{16}$ The 2-pyridinyloxy radical has dominant $\mathrm{C}=\mathrm{N}$ and $\mathrm{C}=\mathrm{C}$ bonds in the ring. The 4-pyridinyloxy radical is more delocalized, and the $\mathrm{C}-\mathrm{N}$ bonds are more similar to the 3-pyridinyloxy radical. Each of these structures has an aza-pentadienyl radical delocalization; however, they differ in the location of the $\mathrm{N}$ atom relative to the unpaired electron. In the 3-pyridinyloxy radical, the unpaired electron cannot be on $\mathrm{N}$, and this confers some stability as the electron-electron repulsion between the $\mathrm{N}$ lone pair (in the $\sigma$ system) and the unpaired electron (in the $\pi$ system) is minimized. 
(a)

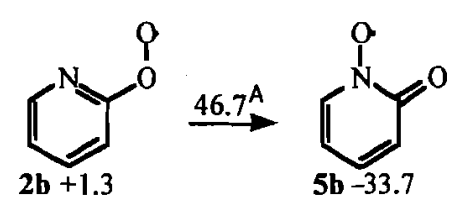

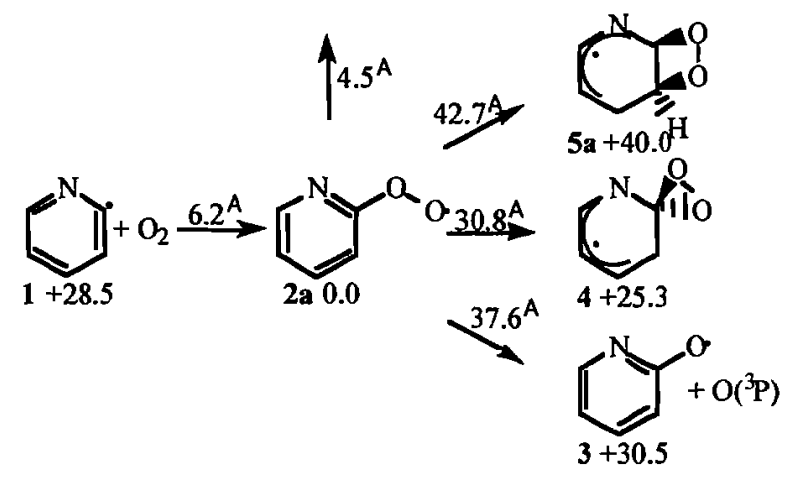

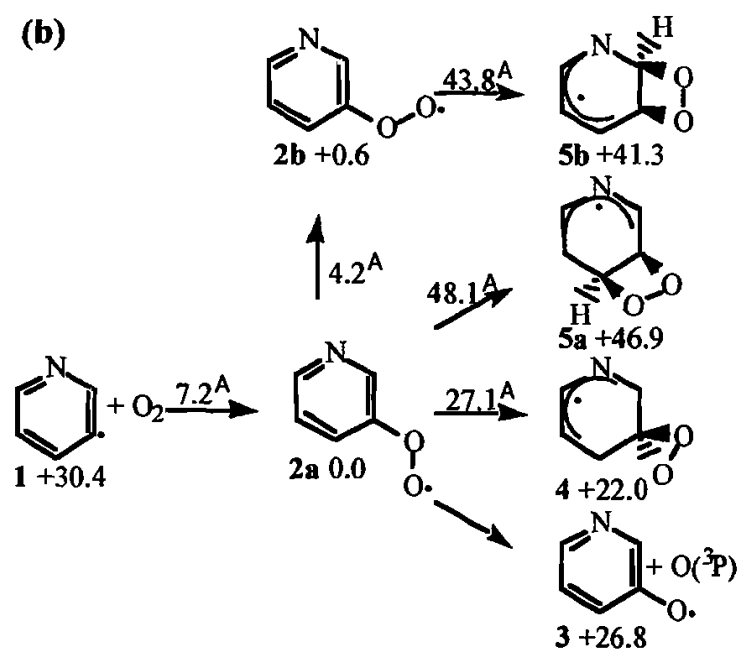

(c)

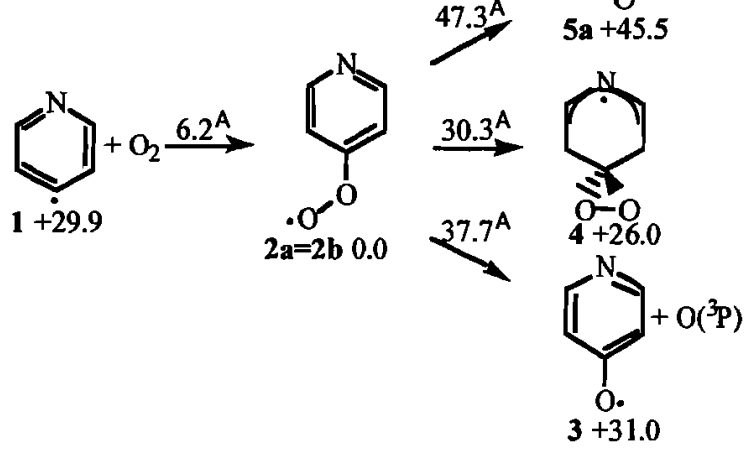

Figure 3.3.2. Mechanisms of the (a) 2-pyridinyl $+\mathrm{O}_{2}$, (b) 3-pyridinyl $+\mathrm{O}_{2}$ and (c) 4-pyridinyl + $\mathrm{O}_{2}$ reactions showing Gibbs free energy of activation barriers (relative to the appropriate reactant) and Gibbs free energies of each intermediate at $298 \mathrm{~K}$ (relative to the corresponding 2a) at the B3LYP/6-311+G**//B3LYP/6-31G* level. 


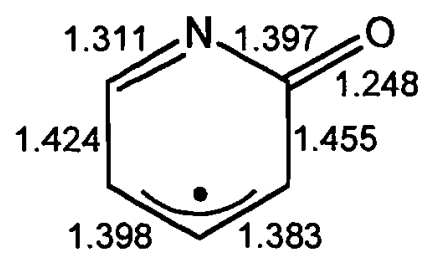

2-pyridinyloxy (3) $C_{\mathrm{s}},{ }^{2} \mathrm{~A}^{\prime \prime}$

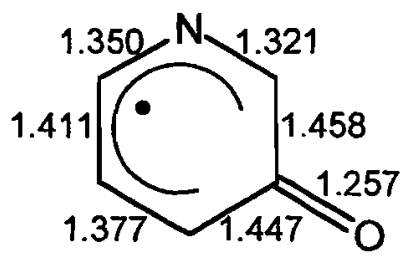

3-pyridinyloxy (3) $C_{\mathrm{s}},{ }^{2} \mathrm{~A} "$

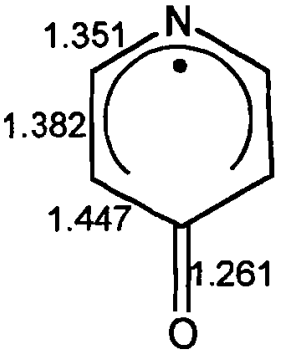

4-pyridinyloxy (3) $C_{\mathrm{s}},{ }^{2} \mathrm{~A}^{\prime \prime}$

Figure 3.3.3. Molecular structures and critical bond lengths $(\AA)$ of the pyridinyloxy radicals (B3LYP/6-31G*).

In the 2- and 4-pyridinyloxy radicals, the unpaired electron can be delocalized onto $\mathrm{N}$. These results are not surprising given that the experimental $\mathrm{O}-\mathrm{H}$ bond dissociation energies of 2-, 3and 4-pyridinol are 99,93 and $100 \mathrm{kcal} / \mathrm{mol}$, respectively. ${ }^{31}$

The differences in stability of these pyridinyloxy intermediates are reflected in the $\Delta G$ $(298 \mathrm{~K})$ for the unimolecular decomposition of the pyridinylperoxy radicals to the pyridinyloxy radical and $\mathrm{O}$ atom (at infinite separation). The endoergicity of this reaction for 3pyridinylperoxy radical is $+26.8 \mathrm{kcal} / \mathrm{mol}$, which is very similar to the $+27.3 \mathrm{kcal} / \mathrm{mol}$ value calculated for the analogous reaction with phenylperoxy radical. This energy is 3 to $4 \mathrm{kcal} / \mathrm{mol}$ more favorable than for the 2- or 4-pyridinylperoxy radicals (Table 3.3.1).

The free energies of activation for the scission of the $\mathrm{O}-\mathrm{O}$ bond in 2- and 4pyridinylperoxy radicals have been determined, but attempts to find the analogous transition state for 3-pyridinylperoxy radical have been fruitless. The $\Delta G^{*}(298 \mathrm{~K})$ for the decomposition of 2- and 4-pyridinylperoxy radical is $\sim 38 \mathrm{kcal} / \mathrm{mol}$ with barriers of $\sim 7 \mathrm{kcal} / \mathrm{mol}$ for the reverse reaction. In an analogous computational study, it was determined that the $\mathrm{O}$-atom addition to vinyloxy radical $\left(\mathrm{CH}_{2}=\mathrm{CHO} \bullet\right)$ to form $\mathrm{CH}_{2}=\mathrm{CHOO} \bullet$ is nearly barrierless. ${ }^{32}$ This observation correlates well with the B3LYP/6-311+G**//B3LYP/6-31G* bottom-of-the-well energy difference of only $\sim 0.1 \mathrm{kcal} / \mathrm{mol}$ between the 2- and 4-pyridinyloxy radicals and $O$ atom 
Table 3.3.1. Gibbs free energies $(298 \mathrm{~K}$ ) for all intermediates and transition states at the B3LYP/6-311+G**//B3LYP/6-31G* level.

\begin{tabular}{lccccccc} 
& 1 & TS 1-2a & 2a & TS 2a-3 & 3 & TS 2a-4 & 4 \\
\hline Phenyl & 32.3 & 38.4 & 0.0 & 51.0 & 27.3 & 27.2 & 22.6 \\
2-Pyridinyl & 28.5 & 34.7 & 0.0 & 37.6 & 30.5 & 30.8 & 25.3 \\
3-Pyridinyl & 30.4 & 37.6 & 0.0 &.--2 & 26.8 & 27.1 & 22.0 \\
4-Pyridinyl & 29.9 & 36.1 & 0.0 & 37.7 & 31.0 & 30.3 & 26.0
\end{tabular}

\begin{tabular}{|c|c|c|c|c|c|c|}
\hline & TS 2a-5a & $5 \mathbf{a}$ & TS 2a-2b & $\mathbf{2 b}$ & TS 2b-5b & $5 \mathbf{b}$ \\
\hline Phenyl & 46.2 & 44.8 & $-a^{6}$ & $--{ }^{6}$ & $--^{6}$ & $--^{6}$ \\
\hline 2-Pyridinyl & 42.7 & 40.0 & 4.5 & 1.3 & 46.7 & -33.7 \\
\hline 3-Pyridinyl & 48.1 & 46.9 & 4.2 & 0.6 & 43.8 & 41.3 \\
\hline 4-Pyridinyl & 47.3 & 45.5 & $--^{b}$ & -.- & $-m^{b}$ & $\ldots$ \\
\hline
\end{tabular}

${ }^{a}$ Despite numerous attempts, this stationary point could not be located at the B3LYP/6-31G* level.

${ }^{6}$ Due to symmetry, $2 \mathrm{a}=\mathbf{2 b}$ and $\mathbf{5 a}=\mathbf{5 b}$.

complexes, as compared to their respective transition states which lead to the peroxy radicals. Also, both calculated transition states suffer from excessive spin contamination $\left(\left\langle S^{2}\right\rangle=1.72\right)$, and these energies are suspect quantitatively.

\subsubsection{Rearrangement of pyridinylperoxy radicals to yield the dioxiranylpyridinyl radical intermediates $(2 \mathbf{a} \rightarrow 4)$}

The isomerization of an arylperoxy radical through the ring closure of the terminal oxygen to the proximal ring carbon (1,1-addition) forming a dioxiranyl intermediate $(4$, Figure 3.3.2) was first proposed by Carpenter ${ }^{14}$ as a possible key step in the unimolecular decomposition of phenylperoxy radical. This proposal was further supported by B3LYP and $\operatorname{CCSD}(\mathrm{T})$ studies, which have shown that the dioxiranyl radical pathway for phenylperoxy radical is favored at temperatures $\leq 1250 \mathrm{~K}^{16}$ Therefore, it is not surprising that the dioxiranyl radical intermediates from the pyridinylperoxy radicals are the most stable at $298 \mathrm{~K}$.

The rearrangement of pyridinylperoxy radical (2) to form the dioxiranyl radical (4) is an endoergic process due to the loss of aromaticity in the pyridinyl ring, but some stability is gained through the delocalization of the electron throughout the ring. The order of stability for the 
dioxiranylpyridinyl radicals is: $3-(+22.0 \mathrm{kcal} / \mathrm{mol})>2-(+25.3 \mathrm{kcal} / \mathrm{mol})>4-(+26.0 \mathrm{kcal} / \mathrm{mol})$, and is related to the ability of each system to delocalize the electron as well as the electron withdrawing nature of the ring nitrogen with respect to the position of the dioxiranyl functional group.

The free energies of activation at $298 \mathrm{~K}$ for forming the dioxiranyl radicals (4) are the lowest of any explored on these potential energy surfaces. $\Delta G^{*}(298 \mathrm{~K})$ is $\sim 30 \mathrm{kcal} / \mathrm{mol}$ for the formation of 2- and 4-dioxiranylpyridinyl radicals, while the barrier is only $\sim 27 \mathrm{kcal} / \mathrm{mol}$ for the 3-dioxiranylpyridinyl radical. Therefore, the rearrangement of the pyridinylperoxy radical (2a) to yield the corresponding dioxiranylpyridinyl radical (4) is the energetically most favorable pathway at $298 \mathrm{~K}$, which is similar to the observations made for phenylperoxy radical.

\subsubsection{Rearrangement of pyridinylperoxy radicals to form a dioxetanylpyridinyl radical intermediates $(2 a \rightarrow 5 a$ or $2 a \rightarrow 2 b \rightarrow 5 b)$}

The 1,2-addition of the terminal oxygen in the pyridinylperoxy radicals to a neighboring carbon or nitrogen atom in the pyridine ring can occur from either the most stable conformation (2a) of the pyridinylperoxy radical or following the rotation $(\mathbf{2} \mathbf{a} \rightarrow \mathbf{2 b})$ of the peroxy group (Figure 3.3.2). The barrier to rotation $\left(\Delta \mathrm{G}^{*}, 298 \mathrm{~K}\right)$ is $\sim 4 \mathrm{kcal} / \mathrm{mol}$ for both 2- and 3pyridinylperoxy radicals, while the activation barrier for dioxetane formation (1,2-addition) ranges from 42.7 to $48.1 \mathrm{kcal} / \mathrm{mol}$ for the formation of all possible dioxetanylpyridinyl radicals.

Formation of the dioxetane ring is endoergic, with $\Delta \mathrm{G}(298 \mathrm{~K})$ ranging from +40.0 to $+46.9 \mathrm{kcal} / \mathrm{mol}$ for all reactions explored except for the one resulting in the 2-pyridinyl-5b intermediate (Figure 3.3.2). The reaction, which yields 2-pyridinyl-5b from 2-pyridinyl-2b, is highly exoergic $(-33.7 \mathrm{kcal} / \mathrm{mol}$ at $298 \mathrm{~K})$. This 1,2 -addition reaction is unique as the activation barrier is similar to the production of the other dioxetanes, but the product gains stability through the scission of the $\mathrm{O}-\mathrm{O}$ bond. The B3LYP/6-31G* optimized geometry of this molecule suggests that the radical is localized on the $\mathrm{O}$ attached to the $\mathrm{N}$ since the $\mathrm{N}-\mathrm{O}$ bond length is 
$1.270 \AA$, which is typical of an $\mathrm{N}-\mathrm{O}$ single bond. (Such nitrosyl radicals are very stable, and are often used as spin-traps in EPR spectroscopy. ${ }^{33}$ )

The reaction mechanism and energetics for NO extrusion from 2-pyridinyl-5b (and related isomers) will be presented in a subsequent paper, ${ }^{34}$ and may proceed in a manner analogous to $\mathrm{CO}$ extrusion from the phenoxy radical as first proposed by Colussi, et al. ${ }^{4}$

Although the dioxetanyl pathway possesses the largest activation barrier of any of the reactions studied here, it cannot be wholly discounted. In our previous study of the unimolecular decomposition of phenylperoxy radical, the dioxetanyl intermediate showed similar energetics (Table 3.3.1), but could readily break the $\mathrm{O}-\mathrm{O}$ bond $\left(\Delta \mathrm{G}^{*}=-0.3 \mathrm{kcal} / \mathrm{mol}\right.$ at $\left.298 \mathrm{~K}\right)$ to form a very stable intermediate $(\Delta \mathrm{G}=-18.0 \mathrm{kcal} / \mathrm{mol}$ at $298 \mathrm{~K}){ }^{16}$ This overall, exoergic process for O-O cleavage, analogous to the one-step reaction which yields 2-pyridinyl-5b, can be expected to occur with similar energetics for all of the dioxetanylpyridinyl radicals.

\subsubsection{Comparison of the reaction pathways as a function of temperature}

Several general trends are observed when the Gibbs free energies and activation barriers are compared at temperatures ranging from 500 to $2000 \mathrm{~K}$ (Tables 3.3.2 and 3.3.3). Based on free energies of activation, the most accessible pathway for the pyridinylperoxy radicals at temperatures $\leq 500 \mathrm{~K}$ is the production of the dioxiranyl intermediate. At temperatures $\geq 1000 \mathrm{~K}$, the loss of $\mathrm{O}_{2}$ from the pyridinylperoxy radical to regenerate the pyridinyl radical has the lowest activation barrier. This feature, especially when coupled with the knowledge that the reaction of pyridinyl radical with $\mathrm{O}_{2}$ at temperatures greater than $1000 \mathrm{~K}$ is endoergic, decreases the likelihood of the pyridinylperoxy radical as an intermediate at high temperatures. Furthermore, at $2000 \mathrm{~K}$, the transition state for loss of $\mathrm{O}_{2}$ has a lower free energy of activation than rotation of the peroxy functional group on the pyridinylperoxy radicals.

Another distinct trend is the similarity of the 3-pyridinyl radical with $\mathrm{O}_{2}$ potential energy surface (PES) with that of the phenyl radical with $\mathrm{O}_{2}$. Notably, the Gibbs free energy of the 3pyridinyloxy or phenoxy radical (3) with $\mathrm{O}$ atom at infinite separation is more stable than the 3pyridinyl or phenyl radical with $\mathrm{O}_{2}$ at temperatures $<1500 \mathrm{~K}$. In stark contrast, 2- and 4- 
pyridinyl radical with $\mathrm{O}_{2}$ complexes are more energetically favored than their pyridinyloxy radical counterpart at all temperatures. This is an important trend since the $\mathrm{C}-\mathrm{H}$ BDEs in pyridine, based on either calculated ${ }^{12}$ or experimental ${ }^{10}$ values, is $2-<4-\approx 3$-pyridinyl radical. As the $\mathrm{C}-\mathrm{H}$ BDE to generate the 2-pyridinyl radical is $\sim 5 \mathrm{kcal} / \mathrm{mol}$ more favored than at the other positions, ${ }^{10,12}$ the trends of the 2-pyridinylperoxy radical are of increased importance. The greatest contrast in the PES of 2-pyridinylperoxy radical versus that of 3- or 4-pyridinylperoxy radicals is that at temperatures as low as $500 \mathrm{~K}$, the free energy of activation for the loss of $\mathrm{O}_{2}$ $(31.0 \mathrm{kcal} / \mathrm{mol})$ is equivalent to the barrier for dioxiranyl formation $(31.1 \mathrm{kcal} / \mathrm{mol})$, while the overall loss of $\mathrm{O}_{2}$ is less endoergic. 
Table 3.3.2. Free energies of activation leading from arylperoxy radicals as a function of temperature at the B3LYP/6-311+G**//B3LYP/6-31G* level.

\begin{tabular}{|c|c|c|c|c|c|c|}
\hline $298 \mathrm{~K}$ & TS 1-2a & TS 2a-2b & TS 2a-3 & TS 2a-4 & TS 2a-5a & TS 2b-5b \\
\hline Phenyl & 38.4 & $-{ }^{a}$ & 51.0 & 27.2 & 46.2 & $\ldots$ \\
\hline 2-Pyridinyl & 34.7 & 4.5 & 37.6 & 30.8 & 42.7 & 46.7 \\
\hline 3-Pyridinyl & 37.6 & 4.2 & $--^{b}$ & 27.1 & 48.1 & 43.8 \\
\hline 4-Pyridinyl & 36.1 & $--^{a}$ & 37.7 & 30.3 & 47.3 & $--^{a}$ \\
\hline $500 \mathrm{~K}$ & TS 1-2a & TS 2a-2b & TS 2a-3 & TS 2a-4 & TS 2a-5a & TS 2b-5b \\
\hline Phenyl & 34.9 & $--^{8}$ & 49.8 & 27.6 & 46.9 & $\ldots \mathbf{a}_{1}$ \\
\hline 2-Pyridinyl & 31.0 & 5.2 & 36.3 & 31.1 & 43.4 & 47.0 \\
\hline 3-Pyridinyl & 34.5 & 5.0 & $\ldots$ & 27.4 & 48.8 & 44.5 \\
\hline 4-Pyridinyl & 32.4 & $\ldots-^{a}$ & 37.1 & 30.5 & 48.0 & $--^{a}$ \\
\hline $1000 \mathrm{~K}$ & TS 1-2a & TS $2 a-2 b$ & TS 2a-3 & TS 2a-4 & TS 2a-5a & TS 2b-5b \\
\hline Phenyl & 26.6 & $\ldots$ & 46.8 & 28.6 & 49.0 & $-^{\mathrm{a}}$ \\
\hline 2-Pyridinyl & 22.1 & 7.6 & 33.0 & 32.3 & 45.5 & 48.3 \\
\hline 3-Pyridinyl & 27.3 & 7.4 & $--^{b}$ & 28.4 & 50.9 & 46.8 \\
\hline 4-Pyridinyl & 23.5 & $--^{\mathrm{a}}$ & 35.9 & 31.5 & 49.9 & $--^{a}$ \\
\hline $1500 \mathrm{~K}$ & TS 1-2a & TS 2a-2b & TS 2a-3 & TS 2a-4 & TS 2a-5a & TS 2b-5b \\
\hline Phenyl & 18.7 & $--^{a}$ & 44.3 & 30.1 & 51.5 & $--^{a}$ \\
\hline 2-Pyridinyl & 13.6 & 10.4 & 30.1 & 33.9 & 48.1 & 50.0 \\
\hline 3-Pyridinyl & 20.5 & 10.3 & $---b$ & 29.8 & 53.4 & 49.5 \\
\hline 4-Pyridinyl & 15.0 & $---^{\mathrm{a}}$ & 35.0 & 32.7 & 52.2 & $--^{a}$ \\
\hline $2000 \mathrm{~K}$ & TS 1-2a & TS 2a-2b & TS 2a-3 & TS 2a-4 & TS 2a-5a & TS 2b-5b \\
\hline Phenyl & 11.0 & $--^{2}$ & 42.0 & 31.8 & 54.2 & $\ldots \mathrm{a}$ \\
\hline 2-Pyridinyl & 5.4 & 13.6 & 27.4 & 35.7 & 50.9 & 51.9 \\
\hline 3-Pyridinyl & 13.9 & 13.6 & $-a^{b}$ & 31.5 & 56.2 & 52.5 \\
\hline 4-Pyridinyl & 6.8 & $--^{a}$ & 34.3 & 34.2 & 54.8 & $--^{\mathrm{a}}$ \\
\hline
\end{tabular}

${ }^{\mathrm{a}}$ Due to symmetry, $\mathbf{2 a}=\mathbf{2 b}$ and $\mathbf{5 a}=\mathbf{5 b}$.

${ }^{b}$ Despite numerous attempts, this stationary point could not be located at the B3LYP/6-31G* level.

\subsubsection{Comparison of theoretical levels}

We have also calculated single point energies at the $\operatorname{UCCSD}(\mathrm{T}) / 6-31 \mathrm{G}^{* *}$ level for 2 pyridinylperoxy radical and its transition states to evaluate the quantitative accuracy of the B3LYP energies. These relative energies (at the bottom of the well) are provided in Table 3.3.4. The UCCSD(T) energies are in good qualitative and quantitative agreement with the B3LYP results, except for structures TS $2 \mathbf{a}-\mathbf{3}$ and TS $\mathbf{2 b - 5 b}$. 

Table 3.3.3. Gibbs free energies ( 298 to $2000 \mathrm{~K}$ ) for all intermediates at the B3LYP/6-311+G**//B3LYP/6-31G* level.

\begin{tabular}{|c|c|c|c|c|c|c|c|}
\hline $298 K$ & $\mathbf{I}$ & $\mathbf{2 a}$ & $2 \mathbf{b}$ & 3 & 4 & $\mathbf{5 a}$ & $\mathbf{5 b}$ \\
\hline Phenyl & 32.3 & 0.0 & $--^{a}$ & 27.3 & 22.6 & 44.8 & $--^{a}$ \\
\hline 2-Pyridinyl & 28.5 & 0.0 & 1.3 & 30.5 & 25.3 & 40.0 & -33.7 \\
\hline 3-Pyridinyl & 30.4 & 0.0 & 0.6 & 26.8 & 22.0 & 46.9 & 41.3 \\
\hline 4-Pyridinyl & 29.9 & 0.0 & $--^{a}$ & 31.0 & 26.0 & 45.5 & $--^{\mathbf{a}}$ \\
\hline 3-Pyridazinyl & 27.1 & 0.0 & 1.1 & 25.3 & 25.9 & 39.6 & -44.6 \\
\hline 4-Pyridazinyl & 26.9 & 0.0 & 0.6 & 28.6 & 23.6 & 45.3 & 42.7 \\
\hline 2-Pyrimidinyl & 28.4 & 0.0 & $--^{a}$ & 34.2 & 28.1 & -33.6 & $--^{a}$ \\
\hline 4-Pyrimidinyl & 26.8 & 0.0 & 1.2 & 34.6 & 29.3 & 40.4 & -33.3 \\
\hline 5-Pyrimidinyl & 28.9 & 0.0 & $\ldots$ & 29.4 & 21.7 & 43.2 & $\ldots$ \\
\hline Pyrazinyl & 26.2 & 0.0 & 0.8 & 28.5 & 22.8 & 35.4 & -34.3 \\
\hline $500 \mathrm{~K}$ & 1 & $\mathbf{2 a}$ & $2 \mathbf{b}$ & 3 & 4 & $\mathbf{5 a}$ & $\mathbf{5 b}$ \\
\hline Phenyl & 24.9 & 0.0 & $--^{a}$ & 20.6 & 22.8 & 45.2 & $--^{a}$ \\
\hline 2-Pyridinyl & 20.5 & 0.0 & 1.1 & 23.8 & 25.5 & 40.5 & -33.6 \\
\hline 3-Pyridinyl & 22.5 & 0.0 & 0.5 & 20.1 & 22.2 & 47.3 & 41.8 \\
\hline 4-Pyridinyl & 22.0 & 0.0 & $\ldots$ & 24.3 & 26.1 & 45.9 & $--^{a}$ \\
\hline 3-Pyridazinyl & 19.2 & 0.0 & 0.4 & 18.4 & 25.8 & 40.1 & -44.4 \\
\hline 4-Pyridazinyl & 19.1 & 0.0 & 0.5 & 21.8 & 23.7 & 45.5 & 43.1 \\
\hline 2-Pyrimidinyl & 20.6 & 0.0 & $--^{a}$ & 27.5 & 28.4 & -33.3 & $--^{a}$ \\
\hline 4-Pyrimidinyl & 18.8 & 0.0 & 1.0 & 27.8 & 29.4 & 40.8 & -33.1 \\
\hline 5-Pyrimidinyl & 21.1 & 0.0 & $--^{a}$ & 23.1 & 21.8 & 43.8 & $--^{8}$ \\
\hline Pyrazinyl & 18.3 & 0.0 & 0.6 & 21.7 & 22.9 & 36.0 & -34.0 \\
\hline $1000 \mathrm{~K}$ & $\mathbf{1}$ & $2 \mathbf{a}$ & $\mathbf{2 b}$ & 3 & 4 & $5 \mathbf{a}$ & $\mathbf{5 b}$ \\
\hline Phenyl & 7.1 & 0.0 & $-^{a}$ & 3.9 & 22.9 & $\overrightarrow{46.0}$ & $--^{8}$ \\
\hline 2-Pyridinyl & 1.9 & 0.0 & 0.5 & 6.8 & 25.5 & 41.4 & -33.2 \\
\hline 3-Pyridinyl & 4.1 & 0.0 & 0.4 & 3.3 & 22.1 & 48.2 & 42.9 \\
\hline 4-Pyridinyl & 3.5 & 0.0 & $--^{a}$ & 7.5 & 26.1 & 46.5 & $\ldots$ \\
\hline 3-Pyridazinyl & 0.5 & 0.0 & -1.5 & 1.1 & 25.3 & 41.1 & -43.9 \\
\hline 4-Pyridazinyl & 0.6 & 0.0 & 0.4 & 4.7 & 23.5 & 46.0 & 43.9 \\
\hline 2-Pyrimidinyl & 2.4 & 0.0 & $\ldots$ & 10.7 & 28.9 & -32.9 & $-a^{a}$ \\
\hline 4-Pyrimidinyl & 0.2 & 0.0 & 0.5 & 10.7 & 29.2 & 41.7 & -32.9 \\
\hline 5-Pyrimidinyl & 3.0 & 0.0 & $--^{2}$ & 7.1 & 21.8 & 45.2 & $\ldots$ \\
\hline Pyrazinyl & -0.1 & 0.0 & 0.0 & 4.7 & 22.9 & 37.4 & -33.5 \\
\hline $1500 \mathrm{~K}$ & 1 & $2 a$ & $\mathbf{2 b}$ & 3 & 4 & $5 \mathbf{a}$ & $5 \mathbf{b}$ \\
\hline Phenyl & -10.1 & 0.0 & $\ldots$ & -12.7 & 22.8 & 46.6 & $-\alpha^{a}$ \\
\hline 2-Pyridinyl & -16.1 & 0.0 & -0.1 & -10.1 & 25.5 & 42.2 & -32.9 \\
\hline 3-Pyridinyl & -13.7 & 0.0 & 0.2 & -13.4 & 21.9 & 48.9 & 43.9 \\
\hline 4-Pyridinyl & -14.4 & 0.0 & $\ldots$ & -9.3 & 25.8 & 46.9 & $\ldots$ \\
\hline 3-Pyridazinyl & -17.6 & 0.0 & -3.5 & -16.2 & 24.6 & 42.0 & -43.3 \\
\hline 4-Pyridazinyl & -17.2 & 0.0 & 0.3 & -12.3 & 23.1 & 46.3 & 44.6 \\
\hline 2-Pyrimidinyl & -15.2 & 0.0 & $\ldots$ & -6.1 & 29.2 & -32.5 & $\ldots$ \\
\hline 4-Pyrimidinyl & -17.9 & 0.0 & -0.1 & -6.5 & 28.9 & 42.4 & -32.7 \\
\hline 5-Pyrimidinyl & -14.5 & 0.0 & $\ldots$ & -8.8 & 21.6 & 46.5 & $\ldots$ \\
\hline Pyrazinyl & -17.8 & 0.0 & -0.5 & -12.3 & 22.7 & 38.8 & -32.9 \\
\hline
\end{tabular}




\begin{tabular}{|c|c|c|c|c|c|c|c|}
\hline $2000 \mathrm{~K}$ & $\mathbf{1}$ & $\mathbf{2 a}$ & $\mathbf{2 b}$ & 3 & 4 & $\mathbf{5 a}$ & $5 \mathbf{b}$ \\
\hline Phenyl & -26.9 & 0.0 & $\cdots a^{a}$ & -29.3 & 22.7 & 47.2 & $--^{a}$ \\
\hline 2-Pyridinyl & -33.6 & 0.0 & -0.7 & -27.0 & 25.3 & 43.0 & -32.6 \\
\hline 3-Pyridinyl & -31.1 & 0.0 & 0.0 & -30.1 & 21.6 & 49.6 & 44.9 \\
\hline 4-Pyridinyl & -31.9 & 0.0 & $--^{\mathbf{a}}$ & -26.1 & 25.5 & 47.3 & $--^{a}$ \\
\hline 3-Pyridazinyl & -35.2 & 0.0 & -5.4 & -33.3 & 23.9 & 42.9 & -42.8 \\
\hline 4-Pyridazinyl & -34.5 & 0.0 & 0.2 & -29.3 & 22.6 & 46.6 & 45.2 \\
\hline 2-Pyrimidinyl & -32.3 & 0.0 & $--^{a}$ & -22.8 & 29.4 & -32.0 & $--^{a}$ \\
\hline 4-Pyrimidinyl & -35.4 & 0.0 & -0.6 & -23.5 & 28.6 & 43.0 & -32.4 \\
\hline 5-Pyrimidinyl & -31.6 & 0.0 & $--^{a}$ & -24.7 & 21.3 & 47.7 & $---^{a}$ \\
\hline Pyrazinyl & -35.1 & 0.0 & -1.0 & -29.3 & 22.4 & 40.1 & -32.4 \\
\hline
\end{tabular}

${ }^{\mathrm{a}}$ Due to symmetry, $\mathbf{2 a}=\mathbf{2 b}$ and $\mathbf{5 a}=\mathbf{5 b}$.

The TS 2a-3 calculations at both the B3LYP and UCCSD(T) levels experience significant spin contamination problems $\left(\left\langle S^{2}\right\rangle=1.79\right.$ and 2.40 , respectively), and these energies are suspect quantitatively. For TS $\mathbf{2 b - 5 b}$, the B3LYP calculation had negligible spin contamination $\left(\left\langle S^{2}\right\rangle=0.76\right)$, while the $\mathrm{UHF} / 6-31 \mathrm{G}^{* *}$ wavefunction had excessive spin contamination $\left(\left\langle S^{2}\right\rangle=1.90\right)$ and also failed to converge the coupled-cluster equations for the UCCSD calculation.

Table 3.3.4. Relative energies $(\mathrm{kcal} / \mathrm{mol})$ at different theoretical levels for a few important intermediates and transition states for 2-pyridinyl radical."

\begin{tabular}{lccccccc} 
& $\mathbf{2 a}$ & TS 2a-2b & TS 1-2 & TS 2a-3 & TS 2a-4 & TS 2a-5a & TS 2b-5b \\
\hline \hline UMP2/6-31G** & 0.0 & 3.1 & 61.7 & 64.0 & 54.5 & 70.6 & 105.6 \\
UMP3/6-31G** & 0.0 & 2.8 & 56.1 & 43.2 & 45.9 & 62.6 & 91.9 \\
UMP4(SDQ)/6-31G** & 0.0 & 3.2 & 51.4 & 40.6 & 41.9 & 57.3 & 86.8 \\
UCCSD/6-31G** & 0.0 & 3.6 & 43.0 & 31.7 & 33.3 & 46.6 & $60.2^{b}$ \\
UCCSD(T)/6-31G** & 0.0 & 4.0 & 44.3 & 36.9 & 32.5 & 44.7 & $-\mathbf{b}^{\mathrm{b}}$ \\
UB3LYP/6-311+G** & $\mathbf{0 . 0}$ & 4.4 & 41.8 & 42.0 & 32.1 & 43.4 & 47.6 \\
UB3LYP/6-31G* & 0.0 & 5.1 & 44.5 & 46.1 & 31.7 & 43.0 & 49.1 \\
\hline UB3LYP/6-311+G** $\left\langle S^{2}\right\rangle$ & 0.76 & $\mathbf{0 . 7 5}$ & 1.76 & 1.79 & 0.78 & 0.77 & 0.76 \\
UCCSD(T)/6-31G** $\left\langle S^{2}\right\rangle$ & 0.77 & 0.76 & 2.40 & 2.40 & 1.43 & 1.43 & 1.90
\end{tabular}

${ }^{a}$ The B3LYP/6-31G* geometry was used in each case and the energies are at the bottom-of-the-well.

${ }^{b}$ The CCSD equations could not be converged to the necessary accuracy $\left(<10^{-7} \mathrm{au}\right)$ by Gaussian 98 in order to complete the UCCSD(T) calculation. The corresponding CCSD energy for $\mathbf{T S} \mathbf{2} \mathbf{b}-\mathbf{5 b}$ reflects a $10^{-5}$ au convergence. 


\subsubsection{Other azabenzenes: pyridazine, pyrimidine and pyrazine}

We have also examined the reactivity (by calculating relevant intermediates) of three diazines: pyridazine (Figure 3.3.4), pyrimidine (Figure 3.3.5), and pyrazine (Figure 3.3.6). In observing trends for these diazines, the best indicator for reactivity of the peroxy radicals are the C-H BDEs from the parent aromatic compound. As noted in previous computational ${ }^{12,35}$ and experimental studies, ${ }^{9,10} \mathrm{H}$-atom abstraction is favored at positions ortho to the ring nitrogen followed by a slight preference for para over meta (Figure 3.3.7). When the C-H BDE is low for generating the parent radical prior to $\mathrm{O}_{2}$ addition, the relative free energies for the decomposition or rearrangement of the peroxy radical are similar to those calculated for the 2-pyridinylperoxy radical. On the contrary, the higher the $\mathrm{C}-\mathrm{H} \mathrm{BDE}$, the more closely the system resembles that of phenylperoxy radical.

The first significant trend is that the loss of $\mathrm{O}_{2}$ from the parent arylperoxy radical is less endoergic than the loss of $O$ atom leading to the aryloxy radical at all temperatures studied for most of the azabenzenes (Figure 3.3.7 and Table 3.3.3). 
(a)

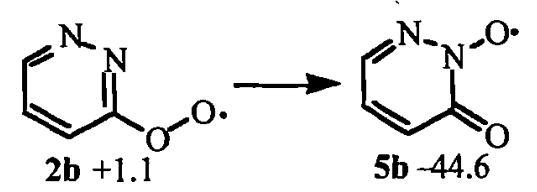

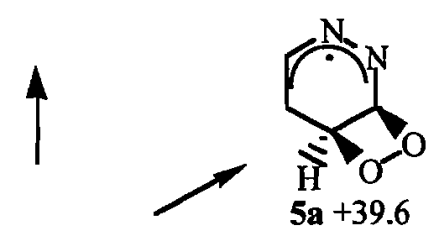

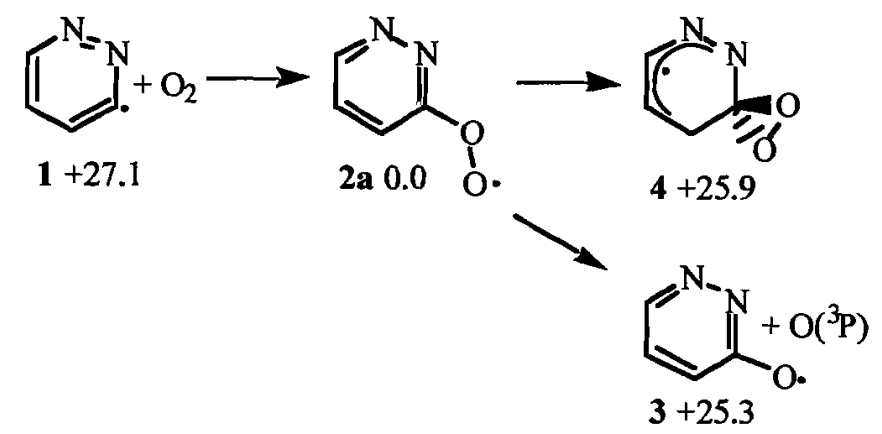

(b)

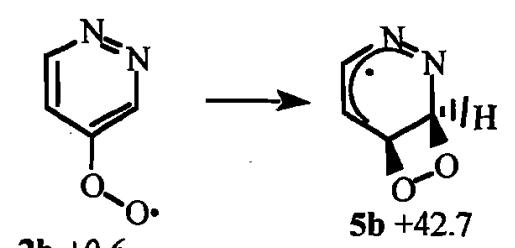<smiles>[124OH]</smiles>

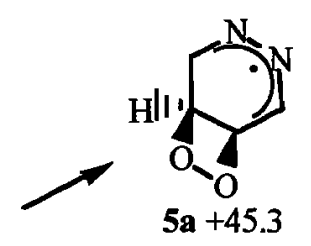<smiles></smiles>

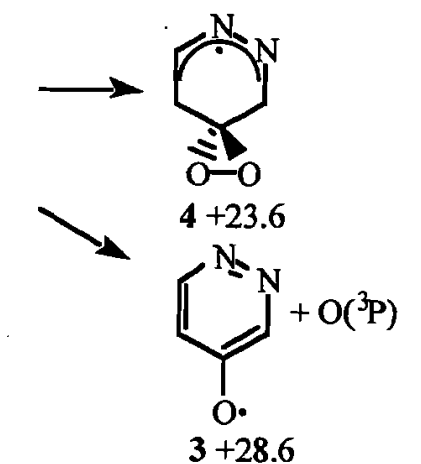

Figure 3.3.4. Mechanisms of the (a) 3-pyridazinyl $+\mathrm{O}_{2}$ and (b) 4-pyridazinyl $+\mathrm{O}_{2}$ reactions showing Gibbs free energies of each intermediate at $298 \mathrm{~K}$ (relative to the corresponding $2 \mathrm{a}$ ) at the B3LYP/6-311+G**//B3LYP/6-31G* level. 

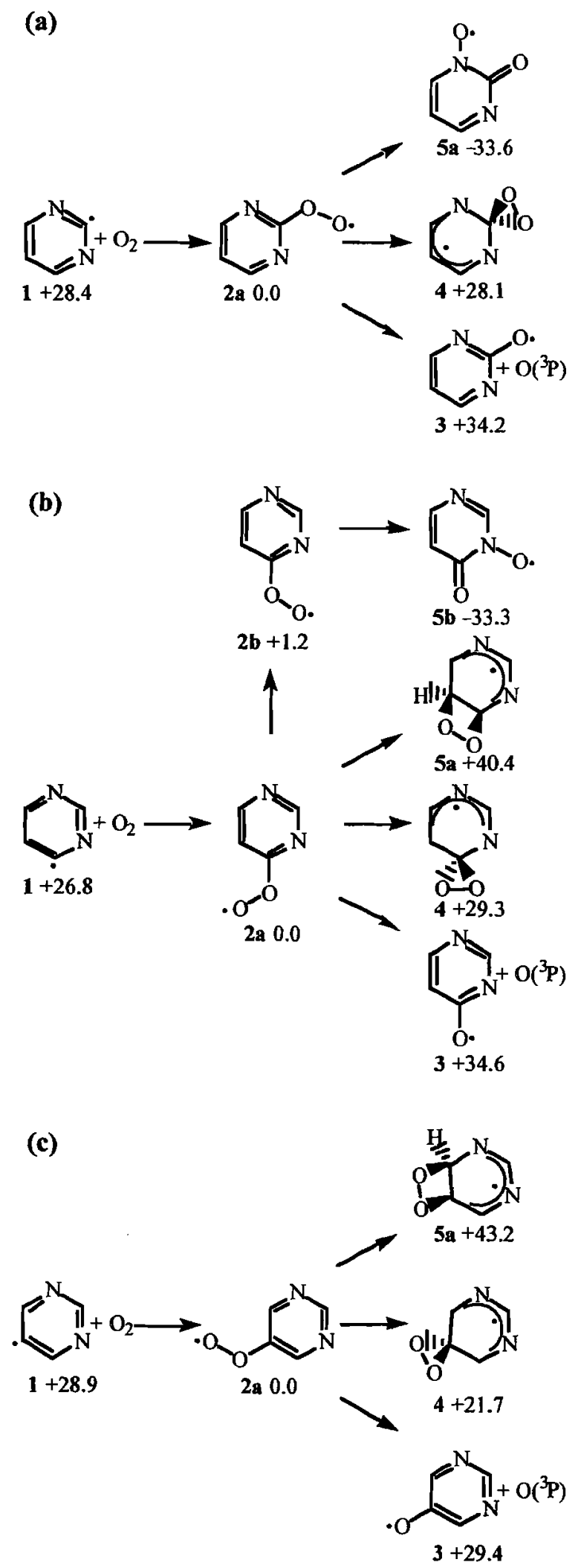

Figure 3.3.5. Mechanisms of the (a) 2-pyrimidinyl $+\mathrm{O}_{2}$, (b) 4-pyrimidinyl $+\mathrm{O}_{2}$ and (c) 5pyrimidinyl $+\mathrm{O}_{2}$ reactions showing Gibbs free energies of each intermediate at $298 \mathrm{~K}$ (relative to the corresponding 2a) at the B3LYP/6-311+G**//B3LYP/6-31G* level. 

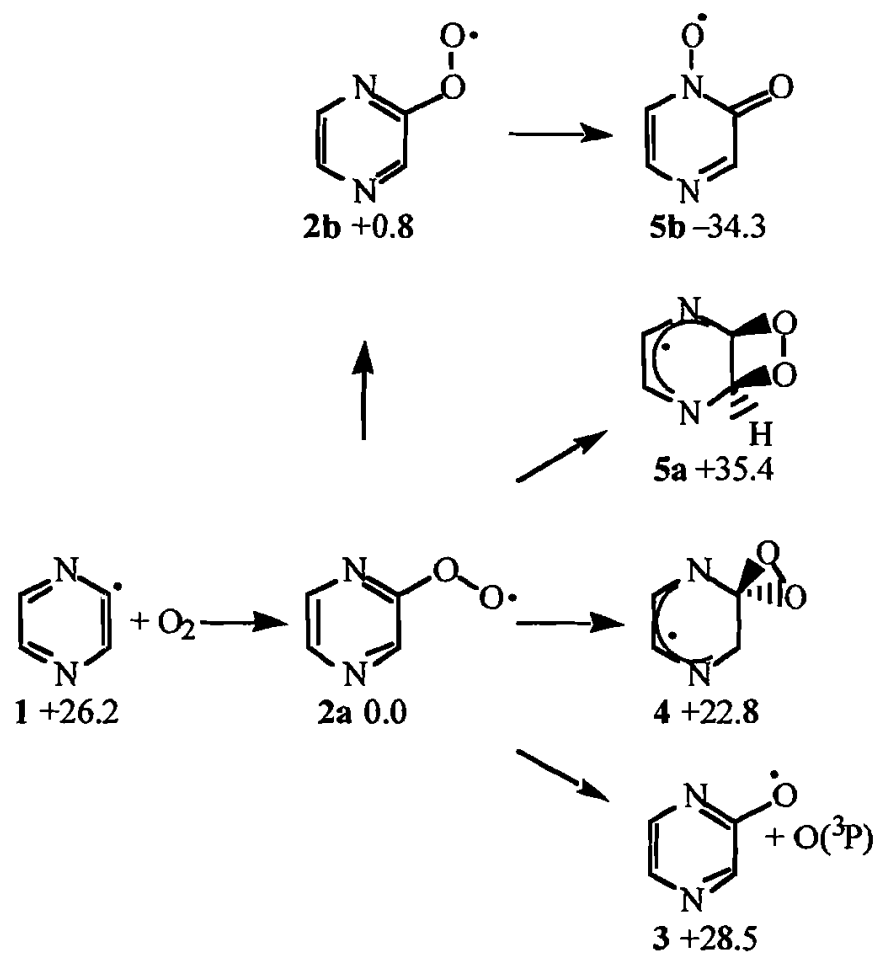

Figure 3.3.6. Mechanism of the pyrazinyl $+\mathrm{O}_{2}$ reaction showing Gibbs free energies of each intermediate at $298 \mathrm{~K}$ (relative to the corresponding 2a) at the B3LYP/6-311+G**//B3LYP/6$31 \mathrm{G}^{*}$ level. 
Increasing C-H Bond Dissociation Enthalpy ${ }^{\mathrm{a}}$

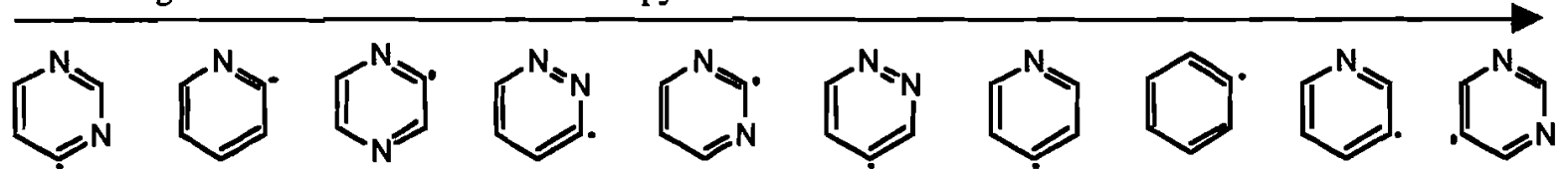

Decreasing preference for $\mathrm{O}_{2}$ loss over $\mathrm{O}$ loss from the arylperoxy radical precursor<smiles>c1cncnc1</smiles><smiles>c1cncnc1</smiles><smiles>c1cnccn1</smiles><smiles>c1ccncc1</smiles><smiles>c1ccnnc1</smiles><smiles>c1ccncc1</smiles><smiles>c1cncnc1</smiles><smiles>c1ccnnc1</smiles>

Decreasing preference for $\mathrm{O}_{2}$ loss over dioxirane formation from the arylperoxy radical precursor<smiles>Ic1ccccc1I</smiles>
$298 \mathrm{~K}$

$500 \mathrm{~K}$

$1000 \mathrm{~K}$

Decreasing preference for $\mathrm{O}$ loss over dioxirane formation from the arylperoxy radical precursor

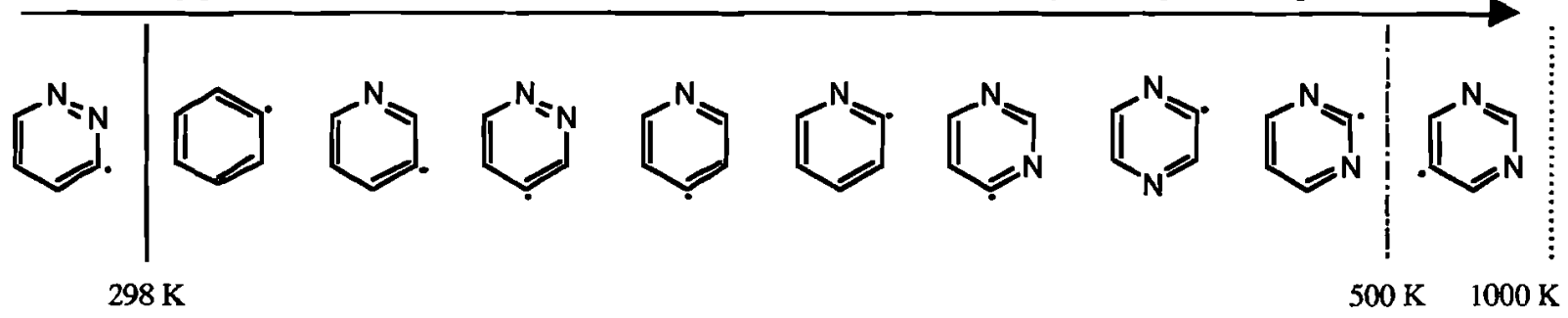

Figure 3.3.7. Observed trends of arylperoxy radical decomposition compared to the related $\mathrm{C}-\mathrm{H}$ $\mathrm{BDE}$ as a function of temperature. Vertical lines represent the temperature of the crossover point for preference of the first pathway over the second. ${ }^{a}$ See ref. 12. 
Exceptions to this trend are the 3-pyridazinylperoxy and 3-pyridinylperoxy radicals. The reasons for the inverse relationship for 3-pyridinylperoxy radical were discussed above, and are not the same causes for the unique stability of 3-pyridazinyloxy radical. Calculations for the 3pyridazinyloxy radical reveal that the most stable electronic state is ${ }^{2} \mathrm{~A}$ ', ie. the unpaired electron is in the $\sigma$ system (Figure 3.3.8). This is in contrast to all of the other aryloxy radicals, for which the ${ }^{2} A^{\prime \prime}(\pi)$ electronic state is more stable than the ${ }^{2} A^{\prime}(\sigma)$ state. Therefore, in the 3pyridazinyloxy radical, the unpaired electron is centered on the proximal ring $\mathrm{N}$ to the carbonyl group in a $\sigma$ orbital so as to maintain the ring's aromatic character $(6 \pi \mathrm{e})$ relative to its 3pyridazinylperoxy radical precursor.

Another predominate trend is that the formation of the dioxiranyl radical intermediate is less endoergic than either $\mathrm{O}_{2}$ loss or $\mathrm{O}$-atom loss at $298 \mathrm{~K}$. The only exceptions to this trend are $\mathrm{O}$-atom loss from the 3-pyridazinylperoxy radical for reasons discussed previously and $\mathrm{O}_{2}$ loss from the 4-pyrimidinylperoxy radical. By $500 \mathrm{~K}$, dioxiranyl radical formation is less favorable than $\mathrm{O}_{2}$ loss or O-atom loss in every azabenzene except for $\mathrm{O}_{2}$ loss from the 3-pyridinylperoxy radical and O-atom loss from 5-pyrimidinylperoxy radical. These observations are depicted in Figure 3.3.7 with vertical lines showing the crossover temperature for the preference of one pathway over the other. The energy difference between the dioxiranyl radicals and the aryloxy radicals with $\mathrm{O}$ atom or the aryl radical with $\mathrm{O}_{2}$ is directly correlated to the respective C-H BDEs of the parent arene (Table 3.3.3 and Figure 3.3.7).

The dioxetanyl radical intermediates are generally the least stable of all studied, and are between 11 to $22 \mathrm{kcal} / \mathrm{mol}$ less stable than their dioxiranyl radical counterparts. Exceptions to this trend are the 3-pyridazinyl-5b, 2-pyrimidinyl-5a, 4-pyrimidinyl-5b and pyrazinyl-5b intermediates which are all stable nitrosyl radicals (Figures 3.3.4 to 3.3.6). These four molecules possess increased stability over their peroxy radical counterparts, ranging from -33.3 to -44.6 $\mathrm{kcal} / \mathrm{mol}(\Delta \mathrm{G}, 298 \mathrm{~K})$. Based on our calculations for the pyridinyl radical system, the transition states connecting the arylperoxy radicals to these four nitrosyl radicals would have an estimated 


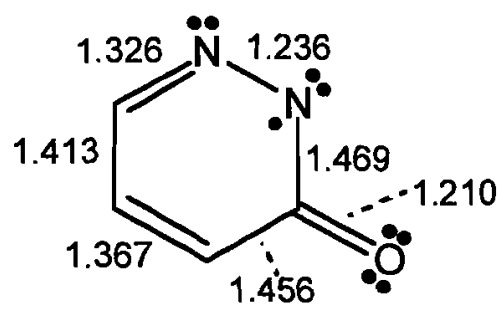

3-pyridazinyloxy (3)

$C_{\mathrm{s}},{ }^{2} \mathrm{~A}^{\prime}$

Figure 3.3.8. Molecular structure and critical bond lengths $(\AA)$ of 3-pyridazinyloxy at the B3LYP/6-31G* level.

free energy of activation of $\geq 45 \mathrm{kcal} / \mathrm{mol}$. Thus, despite the very stable products, the activation barrier is still very substantial and not competitive to other pathways for decomposition.

\subsubsection{Conclusions}

Detailed potential energy surfaces for the reaction of pyridinyl radicals with $\mathrm{O}_{2}$ and subsequent reactions have been presented. Comparisons between the reactivity of pyridinylperoxy and diaza-arylperoxy radicals with the phenylperoxy radical have been addressed. Oxidative mechanisms have been explored through a range of temperatures relevant to atmospheric and combustion processes.

Contrary to phenylperoxy radical decomposition, $\mathrm{O}_{2}$ dissociation from the arylperoxy radical is preferred rather than the loss of $\mathrm{O}$ atom to generate the corresponding aryloxy radical. Formation of dioxiranyl radical intermediates is the most accessible pathway from the peroxy precursor at temperatures $\leq 500 \mathrm{~K}$. Dioxetanyl intermediates are less favored energetically but generate stable nitrosyl radicals which may provide a route to $\mathrm{NO}_{\mathrm{x}}$ generation. 


\section{References for Section 3.3:}

${ }^{1}$ (a) Smith, K. L.; Smoot, L. D.; Fletcher, T. H.; Pugmire, R. J. The Structure and Reaction Processes of Coal; Plenum Press: New York, 1994. (b) Meyers, R. A. Coal Structure; Academic Press: New York, 1982.

${ }^{2}$ Brezinsky, K. Prog. Energy Combust. Sci. 1986, 12, 1-24.

${ }^{3}$ Bittker, D. A. Combust. Sci. and Tech. 1991, 79, 49-72.

${ }^{4}$ Colussi, A. J.; Zabel, F.; Benson, S. W. Int. J. Chem. Kinet. 1977, 9, 161-178.

${ }^{5}$ Zhang, H.-Y.; McKinnon, J. T. Combust. Sci. and Tech. 1995, 107, 261-300.

${ }^{6}$ Chai, Y.; Pfefferle, L. D. Fuel 1998, 77, 313-320.

${ }^{7}$ (a) Nimmo, W.; Richardson, J.; Hampartsoumian, E. J. Inst. Energy 1995, 68, 170-177. (b) Williams, A.; Pourkashanian, M.; Jones, J. M.; Rowlands, L. J. Inst. Energy 1997, 70, 102-113.

${ }^{8}$ Mackie, J. C.; Colket, M. B., III; Nelson, P. F. J. Phys. Chem. 1990, 94, 4099-4106.

${ }^{9}$ Doughty, A.; Mackie, J. C. J. Chem. Soc. Faraday Trans. 1994, 90, 541-548.

${ }^{10}$ Kiefer, J. H.; Zhang, Q.; Kern, R. D.;Yao, J.; Jursic, B. J. Phys. Chem. A 1997, 101, 70617073.

${ }^{11}$ Morris, V.R.; Bhatia, S.C.; Stelson, A. W.; Hall, J. H. Energy \& Fuels 1991, 5, 126-133.

${ }^{12}$ See Barckholtz, C.; Barckholtz, T. A.; Hadad, C. M. J. Am. Chem. Soc. 1999, 121, 491-500, and the references cited therein.

${ }^{13}$ Yu, T.; Lin, M. C. J. Am. Chem. Soc. 1994, 116, 9571-9576.

${ }^{14}$ Carpenter, B. K. J. Am. Chem. Soc. 1993, 113, 9806-9807.

${ }^{15}$ Barckholtz, C.; Fadden, M. J.; Hadad, C. M. J. Phys. Chem. A 1999, 103, 8108-8117.

${ }^{16}$ Fadden, M. J.; Barckholtz, C.; Hadad, C. M. J. Phys. Chem. A 2000, 104, 3004-3011.

${ }^{17}$ (a) Labanowski, J. W.; Andzelm, J. Density Functional Methods in Chemistry; Springer: New York, 1991. (b) Parr, R. G.; Yang, W. Density Functional Theory in Atoms and Molecules; Oxford University Press: New York, 1989.

18 (a) Cioslowski, J.; Liu, G.; Martinov, M.; Piskorz, P.; Moncrieff, D. J. Am. Chem. Soc. 1996, 118, 5261-5264. (b) Cioslowski, J.; Liu, G.; Moncrieff, D. J. Org. Chem. 1996, 61, 4111-4114. ${ }^{19}$ Frisch, M. J.; Trucks, G. W.; Schlegel, H. B.; Gill, P. M. W.; Johnson, B. G.; Robb, M. A.; Cheeseman, J. R.; Keith, T.; Petersson, G. A.; Montgomery, J. A.; Raghavachari, K.; Al-Laham, M. A.; Zakrzewski, V. G.; Ortiz, J. V.; Foresman, J. B.; Peng, C. Y.; Ayala, P. Y.; Chen, W.; Wong, M. W.; Andres, J. L.; Replogle, E. S.; Gomperts, R.; Martin, R. L.; Fox, D. J.; Binkley, J. S.; Defrees, D. J.; Baker, J.; Stewart, J. J. P.; Head-Gordon, M.; Gonzalez, C.; Pople, J. A.

Gaussian 94, Revision D.3, Gaussian, Inc.; Pittsburgh, PA, 1995.

${ }^{20}$ Frisch, M. J.; Trucks, G. W.; Schlegel, H. B.; Scuseria, G. E.; Robb, M. A.; Cheeseman, J. R.; Zakrzewski, V. G.; Montgomery, J. A., Jr.; Stratmann, R. E.; Burant, J. C.; Dapprich, S.; Millam, J. M.; Daniels, A. D.; Kudin, K. N.; Strain, M. C.; Farkas, O.; Tomasi, J.; Barone, V.; Cossi, M.; Cammi, R.; Mennucci, B.; Pomelli, C.; Adamo, C.; Clifford, S.; Ochterski, J.; Petersson, G. A.; Ayala, P. Y.; Cui, Q.; Morokuma, K.; Malick, D. K.; Rabuck, A. D.; Raghavachari, K.;

Foresman, J. B.; Cioslowski, J.; Ortiz, J. V.; Stefanov, B. B.; Liu, G.; Liashenko, A.; Piskorz, P.; Komaromi, I.; Gomperts, R.; Martin, R. L.; Fox, D. J.; Keith, T.; Al-Laham, M. A.; Peng, C. Y.; Nanayakkara, A.; Gonzalez, C.; Challacombe, M.; Gill, P. M. W.; Johnson, B.; Chen, W.; Wong, M. W.; Andres, J. L.; Gonzalez, C.; Head-Gordon, M.; Replogle, E. S.; Pople, J. A. Gaussian 98, Revision A.7, Gaussian, Inc.; Pittsburgh, PA, 1998.

${ }^{21}$ Becke, A. D. Phys. Rev. A 1988, 38, 3098-3100. 
${ }^{22}$ Lee, C.; Yang, W.; Parr, R. G. Phys. Rev. B 1988, 37, 785-789.

${ }^{23}$ Becke, A. D. J. Chem. Phys. 1993, 98, 1372.

${ }^{24}$ Hehre, W. J.; Radom, L.; Schleyer, P. v. R.; Pople, J. A. Ab Initio Molecular Orbital Theory; John Wiley \& Sons: New York, 1986.

${ }^{25}$ Bauschlicher, C. W., Jr.; Langhoff, S. R. Mol. Phys. 1999, 96, 471.

${ }^{26}$ Stanton, J. F.; Gauss, J.; Watts, J. D.; Lauderdale, W. J.; Bartlett, R. J. Int. J. Quantum Chem. $1992, S 26,879$.

${ }^{27}$ Scott, A. P.; Radom, L. J. Phys. Chem. 1996, 100, 16502-16513.

${ }^{28}$ Rablen, P. R., Yale University, 1994.

${ }^{29}$ Chase, M. W., Jr. NIST-JANAF Thermochemical Tables; Washington, DC: American Chemical Society; New York: American Institute of Physics for the National Institute of Standards and Technology, 1998.

${ }^{30}$ Alfassi, Z. B.; Khailkin, G. I.; Neta, P. J. Phys. Chem. 1995, 99, 4544-4548.

${ }^{31}$ Das, T. N.; Neta, P. J. Phys. Chem. A 1998, 102, 7081-7085.

${ }^{32}$ As cited in: Knyazev, V. D.; Slagle, I. R. J. Phys. Chem. 1995, 99, 2247-2249.

${ }^{33}$ (a) Janzen, E. G. Acc. Chem. Res. 1971, 4, 31. (b) Perkins, M. J. Adv. Phys. Org. Chem. 1980, 17, 1. (c) Janzen, E. G.; Evans, C. A.; Davis, E. R. in Organic Free Radicals, ACS Symposium Series, 69; American Chemical Society: Washington, D. C., 1978; p 433.

${ }^{34}$ Fadden, M. J.; Hadad, C. M., manuscript in preparation.

${ }^{35}$ Jones, J.; Backsay, G. B.; Mackie, J. C.; Doughty, A. J. Chem. Soc.Faraday Trans. 1995, 91, $1587-1592$.

\subsection{The Rearrangement Pathways of Arylperoxy Radicals. II. The Five-Membered} Heterocycles

\subsubsection{Executive summary}

The potential energy surfaces for the reaction of furanyl and oxazolyl radicals with $\mathrm{O}_{2}$ have been examined using the B3LYP method. The initial production of the arylperoxy radical followed by either simple decomposition or rearrangement to yield several intermediates (aryloxy, dioxiranylaryl or dioxetanylaryl radicals) has been explored. Transition state structures for most of the steps are presented as well as relative free energies over a range of temperatures from 298 to $2000 \mathrm{~K}$. The energetics of the analogous intermediates for the reaction of $\mathrm{O}_{2}$ and other 5-membered heterocyclic radicals derived from pyrrole and thiophene are also provided. The loss of $\mathrm{O}$ atom is generally the most accessible and energetically favored pathway 
of decomposition at all temperatures. Dioxiranyl formation is favored over $\mathrm{O}_{2}$ loss at temperatures $s 500 \mathrm{~K}$, and favored in the same temperature range over O-atom loss in several cases. Dioxetanyl formation incurs the greatest barrier to formation and direct routes are not available in every molecule surveyed. However, in some cases the dioxetane radicals transform rapidly into very stable species.

\subsubsection{Introduction}

Understanding coal combustion is a challenge because of coal's complex structure and the many functional groups and aromatic rings present in the various ranks of coal. ${ }^{35}$ Many researchers have focused on understanding the behavior of model aromatic rings, such as benzene, pyridine, pyrrole, furan, and thiophene, in order to clarify this complex puzzle. With these model compounds, experimentalists have utilized pyrolysis and oxidative pyrolysis assays in order to provide an understanding of coal combustion.

Many experimental and computational studies have been conducted on the thermal, oxidative pyrolysis of benzene,,$^{35,35,35,35,35}$ but few studies have been conducted on other aromatic compounds. ${ }^{35}$ Of these limited studies, 6-membered monocyclic and bicyclic nitrogencontaining aromatic rings have received some attention. Numerous experimental studies have been reported on the pyrolysis of 5-membered aromatic heterocycles, ${ }^{35,35,35,35,35}$ but none have dealt with oxidative, thermal decomposition. Computational studies ${ }^{35}$ have focused on the decomposition of 5-membered rings either prior to or after $\mathrm{H}$-atom abstraction, but none have dealt with reaction of $\mathrm{O}_{2}$ prior to decomposition.

Recently, we explored the decomposition of phenyl radical with $\mathrm{O}_{2}{ }^{35}$ as well as similar reaction pathways for the analogous decomposition of a wide range of azabenzenes, as depicted in Figure 3.4.1. ${ }^{35}$ Earlier, we presented a thermodynamic study of the decomposition of arylperoxy radicals derived from furan, pyrrole and thiophene to yield $\mathrm{CO}, \mathrm{CO}_{2}$ and other 
smaller hydrocarbons. ${ }^{35}$ However, at that time, we were not able to present a complete potential energy surface for decomposition.

In our continued examination of the reactivity of functional groups present in coal, we now turn our attention to a detailed evaluation of 5-membered heterocyclic rings (Figure 3.4.2). In this study, we examine the potential energy surfaces, including transition states, for the reaction of furan and oxazole with $\mathrm{O}_{2}$ after $\mathrm{H}$-atom abstraction. The thermodynamic free energies from these reaction mechanisms will then be compared to those for thiophene and pyrrole.

\subsubsection{Computational Methods}

All geometry optimizations, vibrational frequency calculations, and single point energy determinations were completed with Gaussian 94 and 98 at the Ohio Supercomputer Center or on our IBM RS/6000 workstations. ${ }^{35,35}$

The B3LYP/6-31G* hybrid density functional theory (DFT) ${ }^{35}$ level was employed to determine the optimized geometries and vibrational frequencies of all stationary points. ${ }^{35,35,35,35}$ We,${ }^{35}$ and others, ${ }^{35}$ have shown DFT methods can be used to provide accurate energies for aromatic radicals. Single point energies were also determined at the B3LYP/6-311+G** level, as Bauschlicher and Langhoff have shown that there is a small basis set effect with oxygencontaining systems when determining $\mathrm{C}-\mathrm{H}$ bond dissociation enthalpies, ${ }^{35}$ so single point energies were also determined at the B3LYP/6-311+G** level in order to provide better relative energies. We have also determined single point energies at the UCCSD(T)/6-31G** level of theory for several key intermediates and transition states in order to compare with our B3LYP results. ${ }^{35}$ All basis sets used 6 cartesian $d$ functions. 


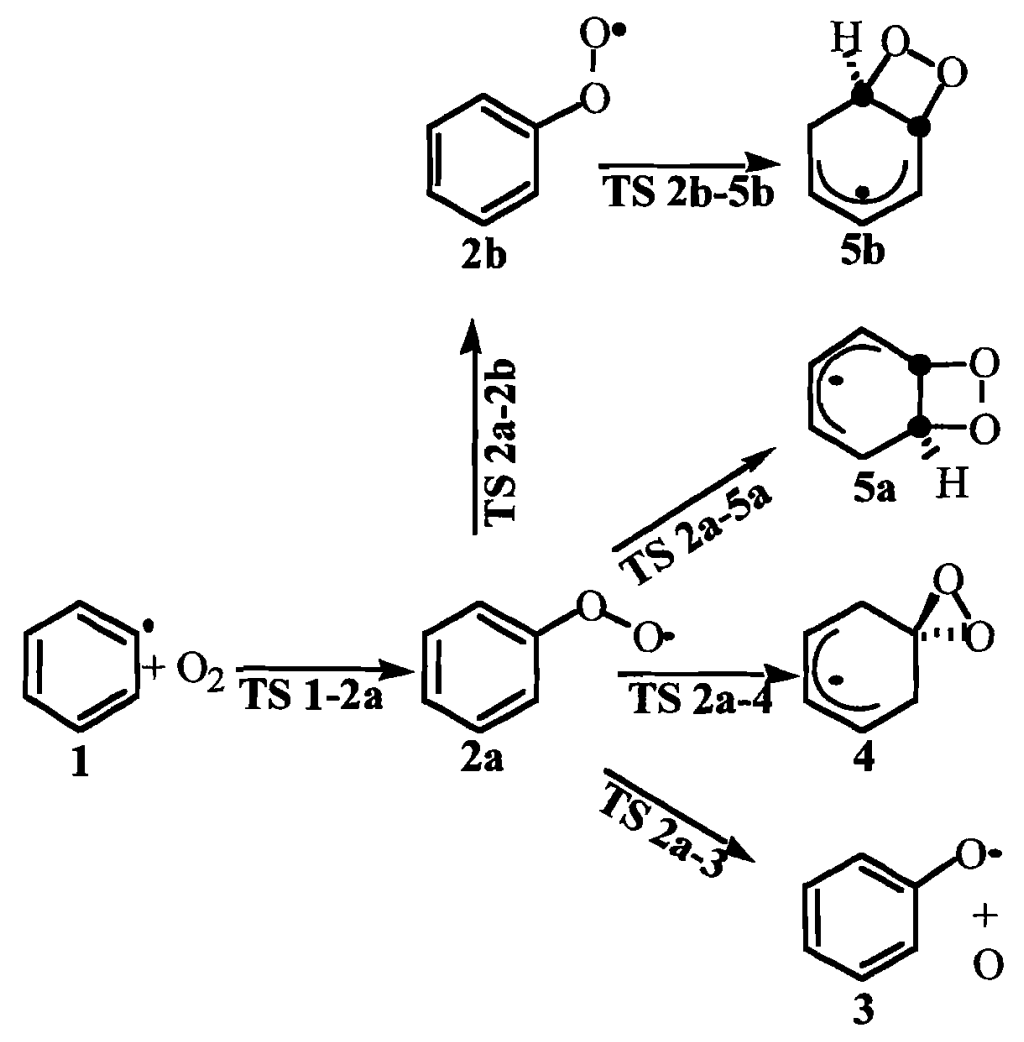

Figure 3.4.1. Reaction mechanism for phenyl radical with $\mathrm{O}_{2}$. This numbering sequence will be used throughout the text, tables and figures to identify the analogous intermediate and transition state for each 5-membered heterocycle.<smiles>c1ccoc1</smiles>

Furan<smiles>c1ccsc1</smiles><smiles>c1cc[nH]c1</smiles>

Pyrrole

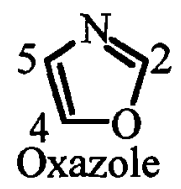

Figure 3.4.2. Molecular structures and numbering of furan, pyrrole, thiophene and oxazole. 
Vibrational frequencies were calculated for each stationary point in order to confirm each structure as a minimum or a saddle point, and each stationary point had the correct number of real and imaginary vibrational frequencies. The zero-point vibrational energy (ZPE) corrections were also obtained and scaled by a factor of $0.9806 .^{35}$ All transition states were confirmed to connect to the corresponding reactant and product by displacing (typically 10\%) the geometry along the reaction coordinate for the imaginary vibrational frequency in either direction, which was then followed by a careful optimization (opt=calcfc or opt=calcall) to the corresponding minimum. In some cases, intrinsic reaction coordinate (IRC) searches were used. ${ }^{35}$ Spin contamination for most of the stationary points is negligible. The $\left\langle S^{2}\right\rangle$ values for all of the minima and for most of the transition states fell below 0.80 for these doublet species. Those specific transition states that suffered from excessive spin contamination will be discussed below, and their energies and geometries are considered to be suspect.

To determine the thermodynamic contribution to the free energy of each molecule at temperatures ranging from 298 to $2000 \mathrm{~K}$, Thermo $94^{35}$ was used to calculate the partition function contributions derived from the optimized geometry and the unscaled vibrational frequencies as calculated by Gaussian. The overall Gibbs free energy at each temperature was derived from the single point energy, the scaled ZPE, the thermodynamic contribution (enthalpy and entropy), and the electronic contribution to the free energy. The only molecule that received different treatment was $\mathrm{O}$ atom, where experimentally ${ }^{35}$ determined splitting energies were included in the electronic component of the partition function for the free energy calculation.

The energies discussed throughout are Gibbs free energies computed at the B3LYP/6-311+G**//B3LYP/631G* level (at 298K, unless noted otherwise), and are relative to the Gibbs free energy of the corresponding arylperoxy radical. 


\subsubsection{Results and Discussion}

\subsubsection{General reaction pathways}

The reaction pathways explored are shown schematically in Figure 3.4.1. The numbering system depicted in Figure 3.4.1 will be used in all of the other figures and throughout the text. (For comparative purposes, we have chosen to use the same numbering system as in our recent paper on the oxidative decomposition of the azabenzenes. ${ }^{15}$ ) The first pathway calculated is $\mathrm{O}_{2}$ addition to the aryl radical (1) in order to form the arylperoxy radical (2). The arylperoxy radical may exist as two distinct isomers ( $2 \mathbf{a}$ and $\mathbf{2 b}$ ), and for our discussion below, the $2 \mathbf{a}$ isomer always represents the more stable isomer. The $\mathbf{2 b}$ isomer will not be shown in cases where the $\mathbf{2 b}$ isomer does not lead to another intermediate or when $\mathbf{2 a}=\mathbf{2 b}$ due to symmetry (as for phenyl radical).

We further explored the fragmentation or unimolecular rearrangement of the arylperoxy radical. Loss of $\mathrm{O}$ atom from the arylperoxy radical yields the aryloxy radical (3) via $\mathrm{O}-\mathrm{O}$ bond cleavage. Unimolecular rearrangement pathways can lead to the dioxiranyl radical (4) or the dioxetanyl radicals (5a and 5b) via 1,1- and 1,2-addition, respectively. We have previously demonstrated that other pathways (such as 1,3- and 1,4-addition) for the decomposition of phenylperoxy radical are highly disfavored due to very large activation barriers. ${ }^{14}$

\subsubsection{Mechanism of the furanyl radical $+\mathrm{O}_{2}$ reaction}

The formation of furanylperoxy radical via the addition of $\mathrm{O}_{2}$ to furanyl radical initiates the process as shown in Figure 3.4.3. Relative energies are provided in Tables 3.4.1 and 3.4.2. At $298 \mathrm{~K}$, this reaction is exoergic for both 2 - and 3-furanyl radicals by -36.1 and $-34.7 \mathrm{kcal} / \mathrm{mol}$, respectively. The free energy of activation $\left(\Delta \mathrm{G}^{\star}, 298 \mathrm{~K}\right)$ for the formation of either the 2- or 3furanylperoxy radical is $\sim 6 \mathrm{kcal} / \mathrm{mol}$. The transition states found for $\mathrm{O}_{2}$ addition to 2- and 3furanyl radical suffer from excessive spin contamination $\left(\left\langle S^{2}\right\rangle \sim 1.8\right)$ and have low-lying imaginary vibrational frequencies ( $19.3 i$ and $41.6 i \mathrm{~cm}^{-1}$, respectively). These values are very 
similar to the previously reported transition state for $\mathrm{O}_{2}$ addition to phenyl radical $\left(\Delta \mathrm{G}^{*}(298 \mathrm{~K})=\right.$ $6.1 \mathrm{kcal} / \mathrm{mol},\left\langle S^{2}>=1.76\right.$, and $\left.6.2 i \mathrm{~cm}^{-1}\right){ }^{14}$

Following the production of 2- or 3-furanylperoxy radical, cleavage of the $\mathrm{O}-\mathrm{O}$ bond can occur to yield the aryloxy radical (3). There is a significant difference in the energetics of this reaction between the 2- and 3-furanylperoxy radicals. The formation of 2-furanyloxy radical (3) is only slightly endoergic at $298 \mathrm{~K}(+3.4 \mathrm{kcal} / \mathrm{mol})$, while production of the 3 -furanyloxy radical undergoes a free energy penalty of $+20.4 \mathrm{kcal} / \mathrm{mol}$ at the same temperature. One previously stated explanation for this variance is the enhanced ability for $\pi$-delocalization by the 2furanyloxy radical over the 3-furanyloxy radical (Figure 3.4.3). ${ }^{16}$

Multiple transition states have been obtained which link the 2-furanylperoxy radical with several different 2-furanyloxy radical with $\mathrm{O}$ atom complexes. The most stable of these requires a free energy of activation barrier of $16.2 \mathrm{kcal} / \mathrm{mol}$ at $298 \mathrm{~K}$ (and a $12.8 \mathrm{kcal} / \mathrm{mol}$ barrier for the reverse reaction). A $27.3 \mathrm{kcal} / \mathrm{mol}$ activation barrier is calculated for the generation of the 3furanyloxy radical (3) via loss of $\mathrm{O}$ atom from the 3-furanylperoxy radical (2a). These two transition states not only differ markedly in their energetic values for the forward and reverse reactions, but also in the geometry of the respective transition states and the magnitude of their imaginary vibrational frequencies. In the 2-furanyloxy case, the $\mathrm{O}$ atom is cleaved perpendicular to the plane of the furan ring and the transition state has an imaginary vibrational frequency of $542 i \mathrm{~cm}^{-1}$. In the 3-furanyloxy case, the $\mathrm{O}$ atom is parallel to the ring with a low-lying imaginary 
(a)
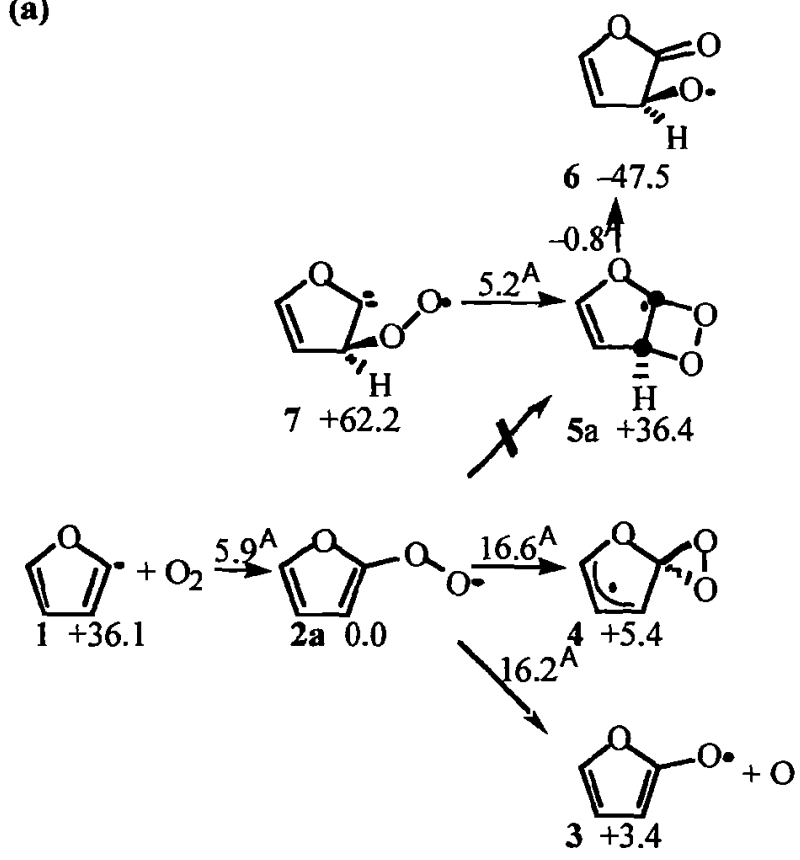

(b)

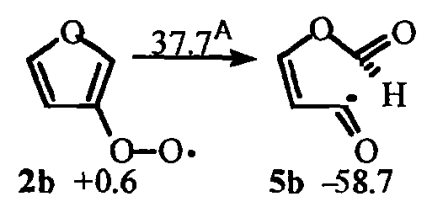
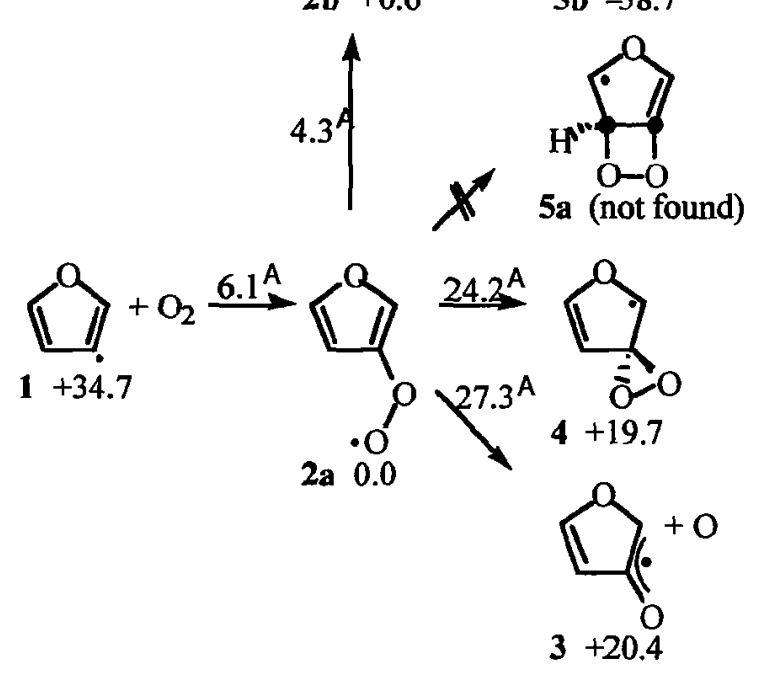

Figure 3.4.3. Mechanisms of the (a) 2-furanyl $+\mathrm{O}_{2}$ and (b) 3-furanyl $+\mathrm{O}_{2}$ reactions showing Gibbs free energy of activation barriers (relative to the appropriate reactant) and Gibbs free energies of each intermediate at $298 \mathrm{~K}$ (relative to the corresponding $2 \mathrm{a}$ ) at the $\mathrm{B} 3 \mathrm{LYP} / 6$ $311+\mathrm{G}^{* *} / / \mathrm{B} 3 \mathrm{~L}$ YP/6-31G* level. 
Table 3.4.1. Gibbs free energies $(\mathrm{kcal} / \mathrm{mol}, 298$ to $2000 \mathrm{~K}$ ) for all intermediates at the B3LYP/6-311+G**//B3LYP/6-31 G* level.

\begin{tabular}{|c|c|c|c|c|c|c|c|}
\hline $298 \mathrm{~K}$ & 1 & $2 a$ & $2 \mathbf{b}$ & 3 & 4 & $5 \mathbf{a}$ & $\mathbf{5 b}$ \\
\hline Phenyl & 32.3 & 0.0 & $\ldots{ }^{8}$ & 27.3 & 22.6 & 44.8 & $--^{a}$ \\
\hline 2-Furanyl & 36.1 & 0.0 & $\ldots$ & 3.4 & 5.4 & 36.4 & $--^{b}$ \\
\hline 3-Furanyl & 34.7 & 0.0 & 0.6 & 20.4 & 19.7 & $--^{c}$ & -58.7 \\
\hline 2-Oxazolyl & 32.3 & 0.0 & 0.5 & 6.3 & 6.8 & -_b & -51.1 \\
\hline 4-Oxazolyl & 34.4 & 0.0 & $-\ldots b$ & 3.8 & 5.8 & 31.6 & $\ldots b$ \\
\hline 5-Oxazolyl & 33.6 & 0.0 & 1.2 & 17.9 & 17.5 & -62.0 & -20.8 \\
\hline Pyrrol-1-yl & -21.1 & 0.0 & $--^{B}$ & 20.8 & 12.5 & 28.1 & $\ldots$ \\
\hline Pyrrol-2-yl & 39.9 & 0.0 & 3.0 & 10.2 & 12.2 & -- & -41.4 \\
\hline Pyrrol-3-yl & 36.6 & 0.0 & 0.2 & 20.8 & 21.2 & -43.8 & -49.5 \\
\hline Thiophen-2-yl & 33.3 & 0.0 & 1.4 & 9.1 & 10.5 & 37.6 & - \\
\hline Thiophen-3-yl & 32.1 & 0.0 & 0.1 & 21.8 & 20.0 & 53.6 & -46.5 \\
\hline $500 \mathrm{~K}$ & 1 & $\mathbf{2 a}$ & $\mathbf{2 b}$ & 3 & 4 & $5 \mathbf{a}$ & $\mathbf{5 b}$ \\
\hline Phenyl & 24.9 & 0.0 & -8 & 20.6 & 22.8 & 45.2 & $-{ }^{\mathrm{a}}$ \\
\hline 2-Furanyl & 28.1 & 0.0 & $--^{b}$ & -3.3 & 5.7 & 36.8 & $\ldots b$ \\
\hline 3-Furanyl & 26.9 & 0.0 & 0.6 & 13.8 & 19.8 & $--^{c}$ & -59.0 \\
\hline 2-Oxazolyl & 24.5 & 0.0 & 0.4 & -0.3 & 7.2 & $--^{b}$ & -50.8 \\
\hline 4-Oxazolyl & 26.6 & 0.0 & $--b$ & -2.8 & 6.1 & 32.2 & $\ldots$ \\
\hline 5-Oxazolyl & 25.7 & 0.0 & 0.8 & 11.3 & 17.6 & -62.3 & -20.7 \\
\hline Pyrrol-1-yl & -28.7 & 0.0 & $-{ }^{a}$ & 14.6 & 12.7 & 28.9 & $\ldots$ \\
\hline Pyrrol-2-yl & 31.5 & 0.0 & 2.7 & 3.4 & 11.7 & $-{ }_{-b}$ & -41.8 \\
\hline Pyrrol-3-yl & 28.7 & 0.0 & 0.2 & 14.3 & 21.1 & -45.7 & -49.7 \\
\hline Thiophen-2-yl & 25.3 & 0.0 & 1.3 & 2.3 & 10.6 & $-b^{\prime}$ & 37.9 \\
\hline Thiophen-3-yl & 24.2 & 0.0 & 0.1 & 15.2 & 20.1 & 53.8 & -47.0 \\
\hline $1000 \mathrm{~K}$ & 1 & $2 \mathbf{a}$ & $2 \mathbf{b}$ & 3 & 4 & $\mathbf{5 a}$ & $\mathbf{5 b}$ \\
\hline Phenyl & 7.1 & 0.0 & $\ldots a$ & 3.9 & 22.9 & 46.0 & $-z^{2}$ \\
\hline 2-Furanyl & 9.5 & 0.0 & $\ldots b$ & -19.8 & 6.0 & 37.8 & $\ldots$ \\
\hline 3-Furanyl & 8.5 & 0.0 & 0.7 & -2.8 & 19.6 & $\ldots$ & -59.7 \\
\hline 2-Oxazolyl & 6.1 & 0.0 & 0.3 & -16.7 & 8.1 & -.-b & -50.0 \\
\hline 4-Oxazolyl & 8.3 & 0.0 & $-{ }^{b}$ & -19.2 & 6.6 & 33.7 & - \\
\hline 5-Oxazolyl & 7.3 & 0.0 & 0.0 & -5.4 & 17.4 & -63.2 & -20.7 \\
\hline Pyrrol-1-yl & -46.5 & 0.0 & $\ldots$ & -0.6 & 12.9 & 30.9 & - \\
\hline Pyrrol-2-yl & 11.9 & 0.0 & 2.1 & -13.4 & 10.1 & $\ldots$ & -43.0 \\
\hline Pyrrol-3-yl & 10.2 & 0.0 & 0.3 & -2.1 & 20.6 & -50.7 & -50.3 \\
\hline Thiophen-2-yl & 6.5 & 0.0 & 1.0 & -14.7 & 10.6 & $-\ldots$ & 38.7 \\
\hline Thiophen-3-yl & 5.7 & 0.0 & 0.2 & -1.4 & 20.2 & 54.2 & -48.3 \\
\hline $1500 \mathrm{~K}$ & 1 & $2 \mathbf{a}$ & $2 \mathbf{b}$ & 3 & 4 & $5 \mathbf{a}$ & $\mathbf{5 b}$ \\
\hline Phenyl & -10.1 & 0.0 & $-n^{a}$ & -12.7 & 22.8 & 46.6 & $-a^{\mathrm{a}}$ \\
\hline 2-Furanyl & -8.5 & 0.0 & $\ldots$ & -36.3 & 6.2 & 38.8 & $\ldots b$ \\
\hline 3-Furanyl & -9.2 & 0.0 & 0.7 & -19.3 & 19.4 & $\ldots c$ & -60.5 \\
\hline 2-Oxazolyl & -11.6 & 0.0 & 0.1 & -33.1 & 8.9 & $--^{b}$ & -49.2 \\
\hline 4-Oxazolyl & -9.4 & 0.0 & $\ldots$ & -35.6 & 7.0 & 35.1 & -.. b \\
\hline 5-Oxazolyl & -10.5 & 0.0 & -0.9 & -22.0 & 17.1 & -64.1 & -20.8 \\
\hline Pyrrol-1-yl & -63.6 & 0.0 & $-a^{a}$ & -15.8 & 13.0 & 32.8 & $\ldots$ \\
\hline Pyrrol-2-yl & -7.0 & 0.0 & 1.5 & -30.2 & 8.4 & $\ldots \mathrm{b}$ & -44.3 \\
\hline Pyrrol-3-yl & -7.6 & 0.0 & 0.4 & -18.4 & 19.9 & -55.6 & -50.9 \\
\hline
\end{tabular}




\begin{tabular}{|c|c|c|c|c|c|c|c|}
\hline Thiophen-2-yl & -11.7 & 0.0 & 0.7 & -31.6 & 10.4 & $\ldots b$ & 39.5 \\
\hline Thiophen-3-yl & -12.2 & 0.0 & 0.3 & -17.9 & 20.1 & 54.5 & -49.6 \\
\hline $2000 \mathrm{~K}$ & 1 & $2 \mathbf{a}$ & $2 \mathbf{b}$ & 3 & 4 & $\mathbf{5 a}$ & $\mathbf{5 b}$ \\
\hline Phenyl & -26.9 & 0.0 & $-a^{a}$ & -29.3 & 22.7 & 47.2 & $-a^{a}$ \\
\hline 2-Furanyl & -26.1 & 0.0 & $\ldots$ & -52.7 & 6.4 & 39.8 & $--^{b}$ \\
\hline 3-Furanyl & -26.5 & 0.0 & 0.8 & -35.6 & 19.0 & $--^{c}$ & -61.4 \\
\hline 2-Oxazolyl & -28.9 & 0.0 & 0.0 & -49.4 & 9.6 & $\ldots b$ & -48.4 \\
\hline 4-Oxazolyl & -26.7 & 0.0 & $\ldots$ & -51.9 & 7.3 & 36.5 & $---b$ \\
\hline 5-Oxazolyl & -27.8 & 0.0 & -1.8 & -38.6 & 16.7 & -65.1 & -20.9 \\
\hline Pyrrol-1-yl & -80.3 & 0.0 & $--^{a}$ & -30.7 & 13.2 & 34.7 & $--^{a}$ \\
\hline Pyrrol-2-yl & -25.5 & 0.0 & 0.9 & -46.9 & 6.6 &.$--b$ & -45.6 \\
\hline Pyrrol-3-yl & -25.0 & 0.0 & 0.5 & -34.6 & 19.1 & -60.7 & -51.5 \\
\hline Thiophen-2-yl & -29.4 & 0.0 & 0.4 & -48.4 & 10.2 & $\ldots \mathrm{b}$ & 40.2 \\
\hline Thiophen-3-yl & -29.5 & 0.0 & 0.3 & -34.3 & 19.9 & 54.8 & -51.0 \\
\hline
\end{tabular}

${ }^{2}$ Due to symmetry, $\mathbf{2 a}=\mathbf{2 b}$ and $\mathbf{5 a}=\mathbf{5 b}$.

${ }^{\mathrm{b}}$ The $2 \mathrm{~b}$ isomers were higher in energy and did not lead to any stable isomers. The $\mathbf{5 a}$ or $\mathbf{5 b}$ isomers are not stable, or an analogous product is simply not possible.

Despite numerous attempts, this stationary point could not be located at the B3LYP/6-31G* level. 
Table 3.4.2. Free energies of activation leading from arylperoxy radicals as a function of temperature at the B3LYP/6-311+G**//B3LYP/6-31G* level.

\begin{tabular}{|c|c|c|c|c|c|c|}
\hline $298 \mathrm{~K}$ & TS 1-2a & TS 2a-2b & TS 2a-3 & TS 2a-4 & TS 2a-5a & TS 2b-5b \\
\hline Phenyl & 38.4 & $--^{a}$ & 51.0 & 27.2 & 46.2 & $\mathrm{Ca}^{\mathrm{a}}$ \\
\hline 2-Furanyl & 42.0 & $\ldots b$ & 16.2 & 16.6 & $\ldots$ & $--^{b}$ \\
\hline 3-Furanyl & 40.8 & 4.3 & 27.3 & 24.2 & $\ldots d$ & 37.7 \\
\hline 2-Oxazolyl & 37.7 & 3.3 & 19.6 & 20.9 & $\ldots$ & $\ldots$ \\
\hline 4-Oxazolyl & 50.3 & $-{ }_{-}^{b}$ & 16.4 & 17.1 & $--^{c}$ & $\ldots b$ \\
\hline 5-Oxazolyl & 49.3 & 3.8 & 25.1 & 24.1 & 37.6 & $\ldots b$ \\
\hline $500 \mathrm{~K}$ & TS 1-2a & TS 2a-2b & TS 2a-3 & TS 2a-4 & TS 2a-5a & TS 2b-5b \\
\hline Phenyl & 34.9 & $\ldots{ }^{8}$ & 49.8 & 27.6 & 46.9 & $\ldots$ \\
\hline 2-Furanyl & 38.1 & $\ldots b$ & 16.0 & 16.9 & $\ldots$ & $\ldots b$ \\
\hline 3-Furanyl & 37.1 & 5.0 & 26.1 & 24.6 & $\ldots{ }^{d}$ & 38.4 \\
\hline 2-Oxazolyl & 33.5 & 4.1 & 19.5 & 21.3 & $--^{b}$ & $\ldots$ \\
\hline 4-Oxazolyl & 46.3 & $\ldots b$ & 16.3 & 17.5 & $\ldots$ & $--^{b}$ \\
\hline 5-Oxazolyl & 45.0 & 4.5 & 24.5 & 24.5 & 38.2 & $\ldots b$ \\
\hline
\end{tabular}

\begin{tabular}{|c|c|c|c|c|c|c|}
\hline $1000 \mathrm{~K}$ & TS 1-2a & TS $2 a-2 b$ & TS 2a-3 & TS 2a-4 & TS 2a-5a & TS $2 b-5 b$ \\
\hline Phenyl & 26.6 & $\ldots$ & 46.8 & 28.6 & 49.0 & $-a^{8}$ \\
\hline 2-Furanyl & 28.6 & $--^{b}$ & 15.8 & 18.1 & $\ldots$ & $--^{b}$ \\
\hline 3-Furanyl & 28.1 & 7.4 & 23.5 & 25.7 & $--^{d}$ & 40.4 \\
\hline 2-Oxazolyl & 23.4 & 6.7 & 19.3 & 22.8 & $--^{b}$ & $\ldots$ \\
\hline 4-Oxazolyl & 36.4 & $--^{b}$ & 16.1 & 18.8 & $\ldots$ & $\ldots$ \\
\hline 5-Oxazolyl & 34.6 & 6.8 & 23.2 & 25.7 & 39.9 & $\ldots$ \\
\hline
\end{tabular}

\begin{tabular}{|c|c|c|c|c|c|c|}
\hline $1500 \mathrm{~K}$ & TS 1-2a & TS 2a-2b & TS 2a-3 & TS 2a-4 & TS 2a-5a & TS 2b-5b \\
\hline Phenyl & 18.7 & $--^{2}$ & 44.3 & 30.1 & 51.5 & $--^{a}$ \\
\hline 2-Furanyl & 19.6 & $\ldots$ & 16.0 & 19.8 & $\ldots$ & $\ldots b$ \\
\hline 3-Furanyl & 19.5 & 10.3 & 21.1 & 27.3 & $\ldots-{ }^{d}$ & 42.8 \\
\hline 2-Oxazolyl & 13.6 & 9.8 & 19.5 & 24.7 & $\ldots$ & $\ldots$ \\
\hline 4-Oxazolyl & 26.9 & $--^{b}$ & 16.3 & 20.6 & -.-c & $---^{b}$ \\
\hline 5-Oxazolyl & 24.7 & 9.7 & 22.2 & 27.3 & 42.0 & $---b$ \\
\hline
\end{tabular}

\begin{tabular}{|c|c|c|c|c|c|c|}
\hline $2000 \mathrm{~K}$ & TS 1-2a & TS $2 a-2 b$ & TS 2a-3 & TS 2a-4 & TS 2a-5a & TS 2b-5b \\
\hline Phenyl & 11.0 & $--^{2}$ & 42.0 & 31.8 & 54.2 & $\ldots{ }^{\mathrm{a}}$ \\
\hline 2-Furanyl & 10.8 & $--^{b}$ & 16.5 & 21.7 & $\ldots c$ & $\ldots b$ \\
\hline 3-Furanyl & 11.2 & 13.5 & 19.1 & 29.2 & --.d & 45.5 \\
\hline 2-Oxazolyl & 4.1 & 13.2 & 20.0 & 26.9 & $\ldots$ & $\ldots$ \\
\hline 4-Oxazolyl & 17.8 & --.b & 16.8 & 22.6 & -..- & ...-b \\
\hline 5-Oxazolyl & 15.0 & 12.9 & 21.6 & 29.2 & 44.4 & $--^{b}$ \\
\hline
\end{tabular}

${ }^{8}$ Due to symmetry, $2 \mathrm{a}=\mathbf{2 b}$ and $5 \mathrm{a}=\mathbf{5 b}$.

${ }^{b}$ Did not search for this transition state because of irrelevance of the product for energetic reasons or an analogous product is simply not possible.

'Numerous transition state searches to link the peroxy precursor and each of these dioxetanes resulted in discovery of transition states for the loss of $O$ atom.

${ }^{d}$ Despite numerous attempts, this stationary point could not be located. 
vibrational frequency of only $15 i \mathrm{~cm}^{-1}$. Both of these transition states suffer from excessive spin contamination with $\left\langle S^{2}\right\rangle$ values of 1.23 and 1.77 for 2 - and 3-furanyloxy radical formation, respectively.

In studying the unimolecular rearrangement of 2- or 3-furanylperoxy radical, we examined two possible transformations (Figure 3.4.3). The bonding of the terminal oxygen to the proximal carbon (1,1-addition) to form the 2- or 3-dioxiranylfuranyl radical (4) was examined. The formation of the 2-dioxiranylfuranyl radical possesses a similar free energy of activation barrier at $298 \mathrm{~K}$ as cleavage of the $\mathrm{O}-\mathrm{O}$ bond but is slightly more endoergic for product formation. On the other hand, formation of the 3-dioxiranylfuranyl radical (2a $\rightarrow$ 4) is not only the more accessible pathway, but is also more favorable energetically than O-atom loss from the 3-furanylperoxy radical $(2 \mathrm{a} \rightarrow 3)$ at $298 \mathrm{~K}$ (Tables 3.4.1 and 3.4.2).

The second rearrangement is the formation of the 2-furanyl-5a and 3-furanyl-5b dioxetanyl radicals (Figure 3.4.3). Although the 2-furanyl-5a species was found as a stable minimum using the B3L YP method, all attempts to find a transition state which linked the 2furanylperoxy radical to the 2-furanyl-5a radical failed. Each search resulted in a transition state for the loss of $\mathrm{O}$ atom from the 2-furanylperoxy radical. Two transition states were found that connect to 2-furanyl-5a as shown in Figure 3.4.3a, but these structures do not link 2-furanyl-5a to the 2-furanylperoxy (2a) radical.

Two dioxetanyl radicals could result from the unimolecular rearrangement of 3furanylperoxy radical (Figure 3.4.3b). The 3-furanyl-5a radical could not be found using the B3LYP method, and all attempts to maintain the 4-membered oxetanyl ring resulted in ring opening and convergence to the 3-furanylperoxy radical. This is not surprising since the 3furanyl-5a product would be an anti-Bredt ${ }^{35}$ bicyclic system as shown in part c of Figure 3.4.4, and would be unable to delocalize effectively the radical's spin density. The only confirmed dioxetanyl transition state for the rearrangement of a furanylperoxy radical results in the 
(a)

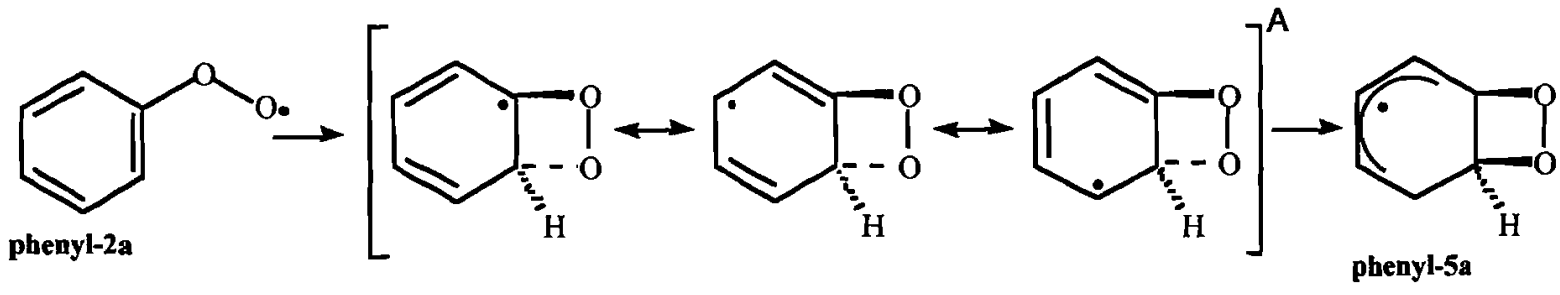

(b)

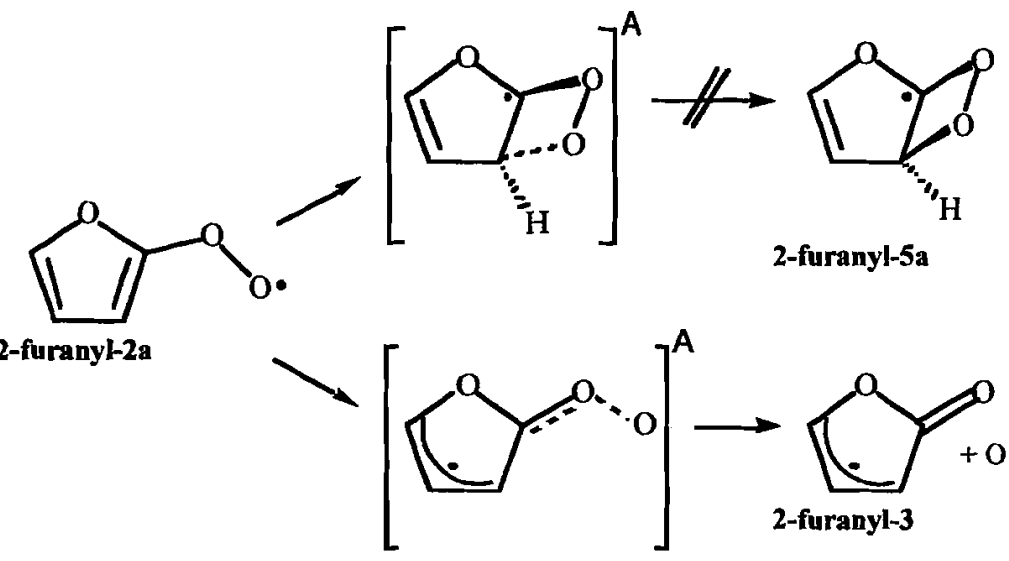

(c)
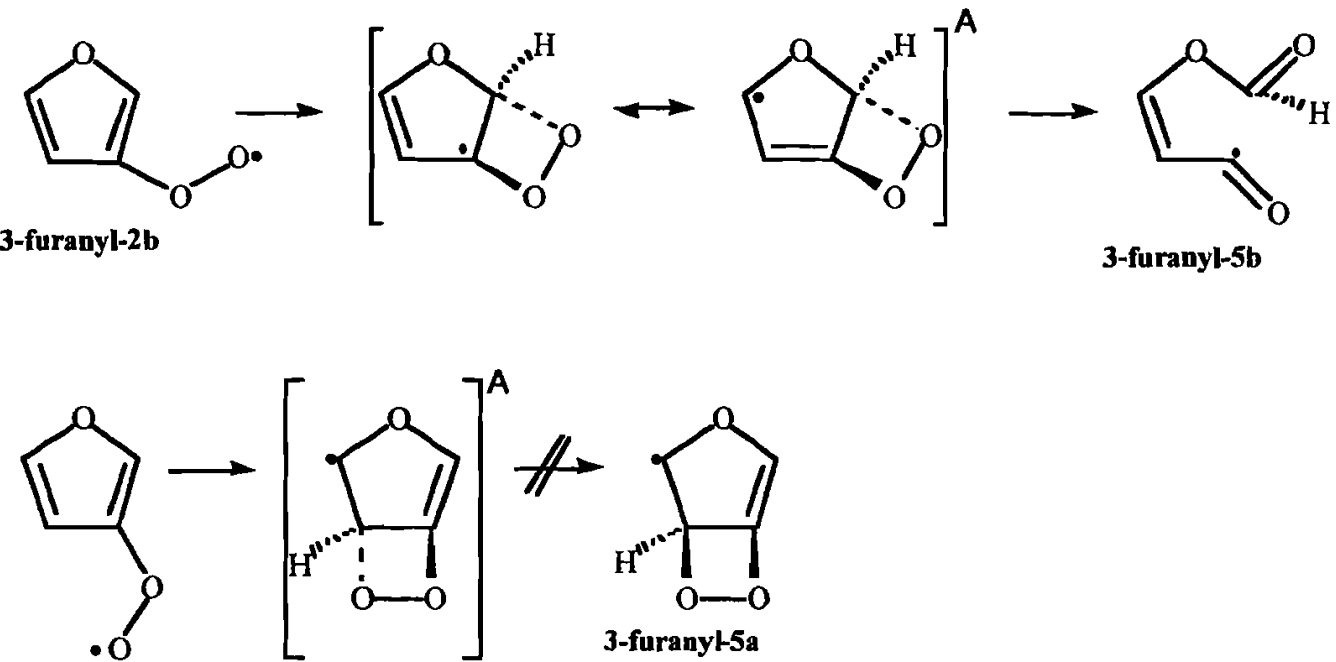

3-furanyl-2a

Figure 3.4.4. Pictorial representation of the possible unimolecular rearrangements of (a) phenylperoxy, (b) 2-furanylperoxy and (c) 3-furanylperoxy radicals to yield dioxetanyl intermediates. Transition state geometries with resonance structures are also depicted and marked with * 
production of the 3-furanyl-5b radical. The free energy of activation barrier is $\sim 5$ to $10 \mathrm{kcal} / \mathrm{mol}$ lower than that seen in the pheny $l^{16}$ or pyridiny $l^{15}$ potential energy surfaces for the analogous reactions. The production of 3 -furanyl-5b radical is significantly exoergic even though it possesses the highest activation barrier of these considered pathways. This effect is due to the concerted nature of the isomerization where the transfer of an $\mathrm{O}$ atom is coupled to the breaking of the $\mathrm{C}_{2}-\mathrm{C}_{3}$ bond in the 3-furanyl-5b radical.

In general, there is a direct correlation between the ability of each system to delocalize the radical center and the discovery of a transition state structure (Figure 3.4.4). Phenylperoxy radical readily rearranges to form a dioxetanyl intermediate, yet no transition state has been discovered which links 2-furanylperoxy radical (2a) with 2-furanyl-5a. However, the connection of 3-furanylperoxy radical to 3-furanyl-5b has been established (Figure 3.4.4c). This transition state and the one for phenylperoxy radical rearrangement can effectively delocalize the radical around the ring, while the 2-furanylperoxy radical is not afforded this ability. In what has been seen in the furanyl and will be shown in the oxazolyl system, dioxetane formation appears to be significantly dependent on delocalization of the radical in the intermediate and also in the transition state.

\subsubsection{Comparison to the oxazolyl radical $+\mathrm{O}_{2}$ reaction mechanism}

The oxidation of 2-, 4-, and 5-oxazolyl radicals and further rearrangement was studied to determine the effect of a second heteroatom within a 5-membered ring (Figure 3.4.2). In particular, oxazole allows for an examination of the effect of $\mathrm{N}, \mathrm{O}$ or both heteroatoms on the relative rearrangement pathways. For instance, the 5-position of oxazole is ortho only to $\mathrm{N}$, while the 4-position is ortho only to $\mathrm{O}$. The 2-position is ortho to both $\mathrm{N}$ and $\mathrm{O}$, and a mechanistic analysis allows for an examination of any synergistic effects. However, in general, the energetics of the intermediates and transition states of the oxazolylperoxy radical (2) are very well correlated with those for the furanylperoxy radicals (Tables 3.4.1 and 3.4.2). 

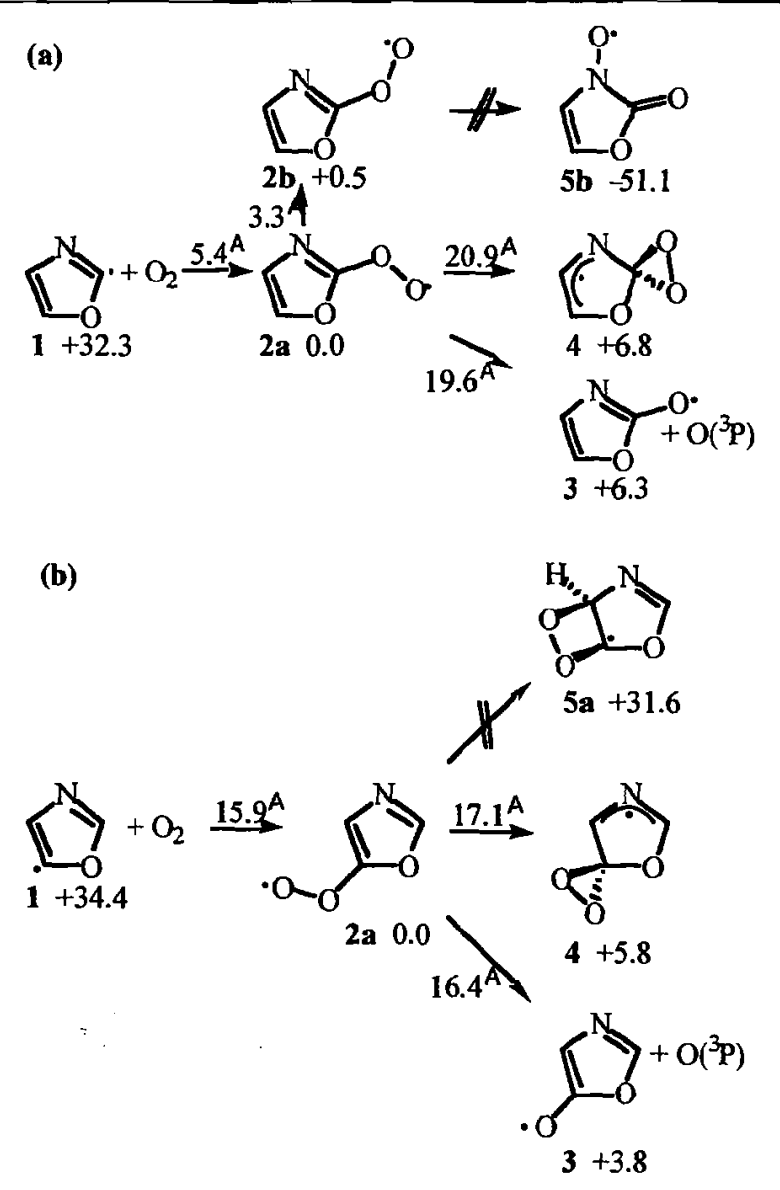

(c)
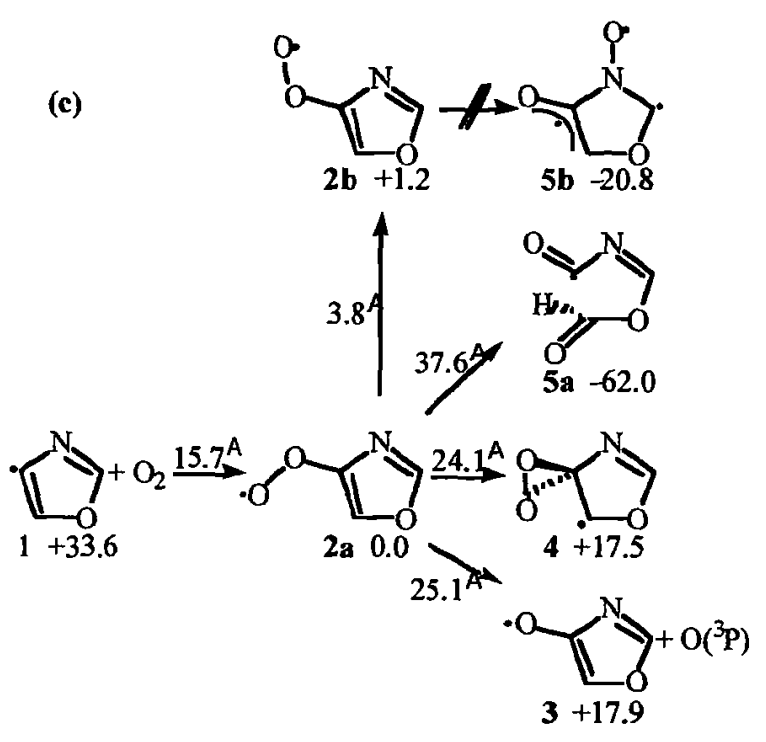

Figure 3.4.5. Mechanisms for the (a) 2-oxazolyl $+\mathrm{O}_{2}$, (b) 4-oxazolyl $+\mathrm{O}_{2}$ and (c) 5-oxazolyl + $\mathrm{O}_{2}$ reactions showing Gibbs free energy of activation barriers (relative to the appropriate reactant) and Gibbs free energies of each intermediate at $298 \mathrm{~K}$ (relative to the corresponding 2a) at the B3LYP/6-311+G**//B3LYP/6-31G* level. 
The rearrangement of the 2- and 4-oxazolylperoxy radicals closely follow all of the major trends seen in the 2-furanylperoxy radical's rearrangements (Figure 3.4.5). For both 2- and 4oxazolylperoxy radicals, the least endoergic reaction at $298 \mathrm{~K}$ is the loss of $\mathrm{O}$ atom to yield the 2and 4-oxazolyloxy (3) radicals. The cleavage of the $\mathrm{O}-\mathrm{O}$ bond also has the lowest calculated free energy of activation barrier $\left(\Delta \mathrm{G}^{*}, 298 \mathrm{~K}\right)$, but these calculations are suspect due to the excessive spin contamination. The presence of the $\mathrm{N}$ atom in the ring raises slightly the calculated energies at the 2-position compared to the 4-position or even those of the 2-furanyl system. Another similarity is seen in the search for transition states that lead to formation of the possible dioxetanyl radicals, 2-oxazolyl-5b and 4-oxazolyl-5a. In both cases, all attempts failed; transition states found during these searches linked the 2- and 4-oxazolylperoxy radicals to 2and 4-oxazolyloxy (3) radicals.

The 5-oxazolylperoxy radical pathways mirror that of the 3-furanylperoxy radical (Figure 3.4.5c). The activation barriers and endoergicities are slightly lowered for the reactions that form the 5-oxazolyloxy radical (3) and the 5-dioxiranyloxazolyl radical (4) as compared to the 3furanylperoxy potential energy surface. In general, the 5-oxazolylperoxy radical has higher barriers for rearrangement as compared to the 2- and 4-oxazolylperoxy radicals. However, for both 3-furanylperoxy and 5-oxazolylperoxy radicals, the dioxiranyl pathways are favored at $298 \mathrm{~K}$ over all of the other rearrangements examined.

\subsubsection{Comparison of theoretical levels}

We also calculated single point energies at the UCCSD(T)/6-31G** level for the 3furanylperoxy radical, 5-oxazolylperoxy radical and their transition states for rearrangement in order to evaluate the quantitative accuracy of the B3LYP energies. These relative energies (at the bottom of the well) are provided in Table 3.4.3. The UCCSD(T) energies are in good qualitative and quantitative agreement with the B3LYP results (within $\sim 3 \mathrm{kcal} / \mathrm{mol}$ ). Due to the high level of spin contamination for the UHF reference wave function, the 3-furanyl-TS 2a-3 and the 5- 
oxazolyl-TS 2a-3 calculations at the UCCSD(T) level failed to converge the coupled-cluster equations. However, overall there is general consensus between the B3LYP and UCCSD(T) energies.

Table 3.4.3. Relative energies $(\mathrm{kcal} / \mathrm{mol})$ at different theoretical levels for a few important intermediates and transition states on the potential energy surface of 3-furanylperoxy and 5-oxazolylperoxy radicals."

\begin{tabular}{llccccc} 
3-furanylperoxy radical & 2a & TS 1-2a & TS 2a-2b & TS 2a-3 & TS 2a-4 & TS 2b-5b \\
\hline \hline UMP2/6-31G** & 0.0 & 44.9 & -5.0 & 54.8 & 26.0 & 56.4 \\
UMP3/6-31G** & 0.0 & 46.2 & -3.6 & 43.7 & 26.7 & 51.6 \\
UMP4(SDQ)/6-31G** & 0.0 & 44.8 & -1.6 & 44.0 & 26.3 & 48.1 \\
UCCSD/6-31G** & 0.0 & 44.1 & 2.0 & $35.3^{\mathrm{b}}$ & 25.5 & 38.6 \\
UCCSD(T)/6-31G** & 0.0 & 45.6 & 2.4 & $-\mathrm{B}^{\mathrm{b}}$ & 24.0 & 35.8 \\
UB3LYP/6-311+G** & 0.0 & 47.9 & 4.1 & 31.1 & 25.5 & 38.5 \\
UB3LYP/6-31G* & 0.0 & 50.2 & 4.5 & 35.3 & 25.5 & 38.2 \\
\hline UB3LYP/6-311+G** $\left\langle S^{2}\right\rangle$ & 0.76 & 1.77 & 0.75 & 1.77 & 0.77 & 0.78 \\
UCCSD(T)/6-31G** $\left\langle S^{2}\right\rangle$ & 0.95 & 2.16 & 0.77 & 2.02 & 1.09 & 1.41
\end{tabular}

\begin{tabular}{lcccccc} 
5-oxazolylperoxy radical & 2a & TS 1-2a & TS 2a-2b & TS 2a-3 & TS 2a-4 & TS 2a-5a \\
\hline \hline UMP2/6-31G** & 0.0 & 48.0 & 2.5 & 39.0 & 29.3 & 64.4 \\
UMP3/6-31G** & 0.0 & 49.0 & 1.8 & 28.2 & 29.2 & 57.5 \\
UMP4(SDQ)/6-31G** & 0.0 & 47.1 & 2.4 & 28.4 & 28.5 & 53.2 \\
UCCSD/6-31G** & 0.0 & 45.1 & 2.6 & $25.7^{\text {b }}$ & 26.8 & 40.3 \\
UCCSD(T)/6-31G** & 0.0 & 46.3 & 2.8 & $-\mathrm{b}^{*}$ & 25.0 & 37.0 \\
UB3LYP/6-311+G** & 0.0 & 57.2 & 3.5 & 28.0 & 25.3 & 38.7 \\
UB3LYP/6-31G* & 0.0 & 60.0 & 4.1 & 32.2 & 25.4 & 38.3 \\
\hline UB3LYP/6-311+G** $\left\langle S^{2}\right\rangle$ & 0.76 & 1.76 & 0.75 & 1.49 & 0.77 & 0.78 \\
UCCSD(T)/6-31G & 0.87 & 2.11 & 0.86 & 1.93 & 1.04 & 1.44
\end{tabular}

${ }^{8}$ The B3LYP/6-31G* geometry was used in each case. The relative energies are at the bottom-of-the-well. ${ }^{b}$ The CCSD equations could not be converged to the necessary accuracy $\left(<10^{-7}\right.$ au $)$ by Gaussian 98 in order to complete the UCCSD(T) calculation. The corresponding CCSD energy for TS $2 \mathrm{a}-3$ reflect a $10^{-5}$ au convergence.

\subsubsection{Comparison of reaction pathways as a function of temperature}

Upon observing the changes in relative Gibbs free energy as the temperature increases from 298 to 2000K, several trends become evident (Tables 3.4.1 and 3.4.2). At temperatures $<1500 \mathrm{~K}$, the addition of $\mathrm{O}_{2}$ to the furanyl or oxazolyl radicals is exoergic, and demonstrates that the formation of the arylperoxy intermediate is thermodynamically favorable across a wide range of temperatures. At temperatures $>1500 \mathrm{~K}$, if the arylperoxy radical intermediate is formed, then 
the simple decomposition of arylperoxy to aryl radical and $\mathrm{O}_{2}$ has the lowest activation barrier, except for the 4-oxazolylperoxy radical.

Another important trend is the proclivity of the arylperoxy radicals derived from the 5membered rings to lose an $\mathrm{O}$ atom at temperatures $\geq 500 \mathrm{~K}$. In general, the furanyloxy and oxazolyloxy radicals (3) are the most stable intermediates from 500 to $2000 \mathrm{~K}$, except for a few dioxetanyl radical species. These dioxetanyl radicals include 3-furanyl-5b, 2-oxazolyl-5b, 5oxazolyl-5a, and 5-oxazolyl-5b (Figures 3.4.3 - 3.4.5); however, in these peculiar cases, either the activation barriers for formation of the oxetanyl radicals are at least $10 \mathrm{kcal} / \mathrm{mol}$ higher than the loss of $\mathrm{O}$ atom or transition states were not found for the direct conversion of the peroxy radical precursor to yield the dioxetanyl radical intermediate. The production of the dioxiranyl radical intermediates is only favored kinetically or thermodynamically at low temperatures ( $\leq 500$ K) for the 3-furanyl and 5-oxazolyl potential energy surfaces.

\subsubsection{Other 5-membered rings: pyrrole and thiophene}

We also calculated the analogous intermediates (but not their respective transition states) for the pyrrolyl and thiophenyl radicals (Table 3.4.1 and Figures 3.4.6 and 3.4.7). In comparing the energetics for these systems with those of the phenyl, furanyl and oxazolyl radicals, several trends become evident. These trends are shown schematically in Figure 3.4.8. The trends are consistently related to the position of the radical center relative to the heteroatom (2- vs 3position) as well as the magnitude of the $\mathrm{C}-\mathrm{H}$ bond dissociation enthalpy (BDE) for generating the carbon-centered radical. ${ }^{24}$ 

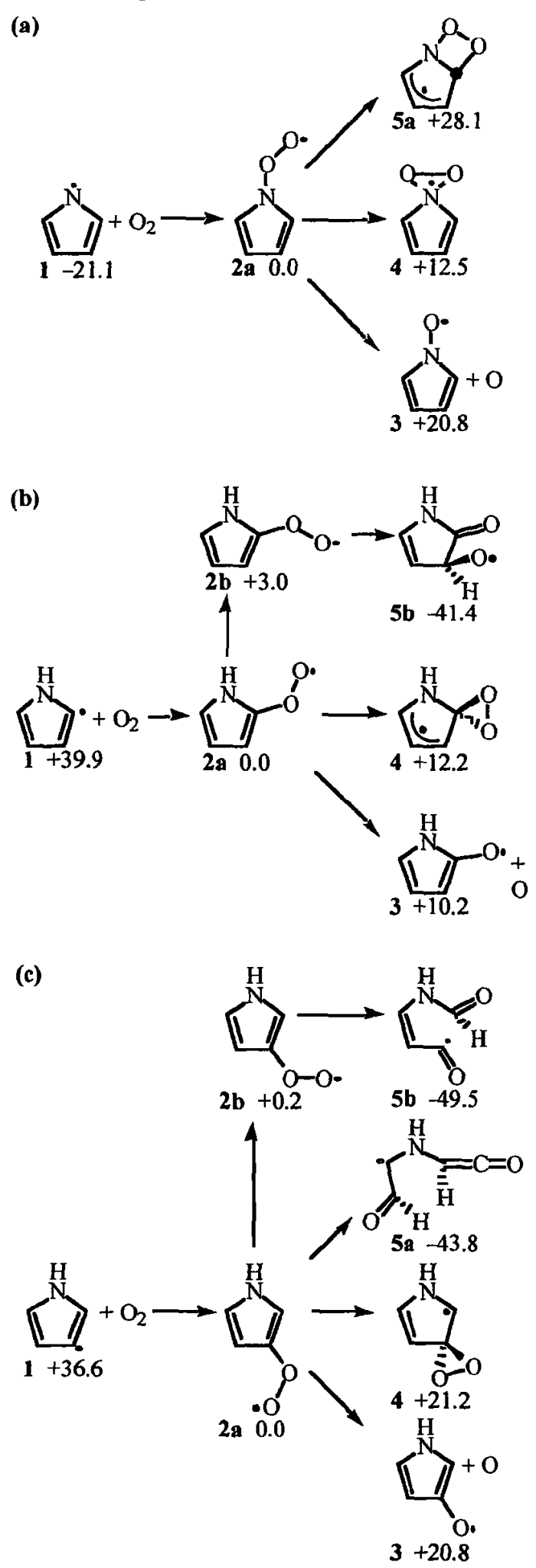

Figure 3.4.6. Mechanisms for the (a) pyrrol-1-yl $+\mathrm{O}_{2}$, (b) pyrrol-2-yl $+\mathrm{O}_{2}$ and (c) pyrrol-3-yl + $\mathrm{O}_{2}$ reactions showing Gibbs free energies of each intermediate at $298 \mathrm{~K}$ (relative to the corresponding 2a) at the B3L YP/6-311+G**//B3LYP/6-31G* level. 
(a)

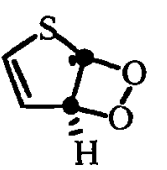

$\mathbf{5 a}+37.6$

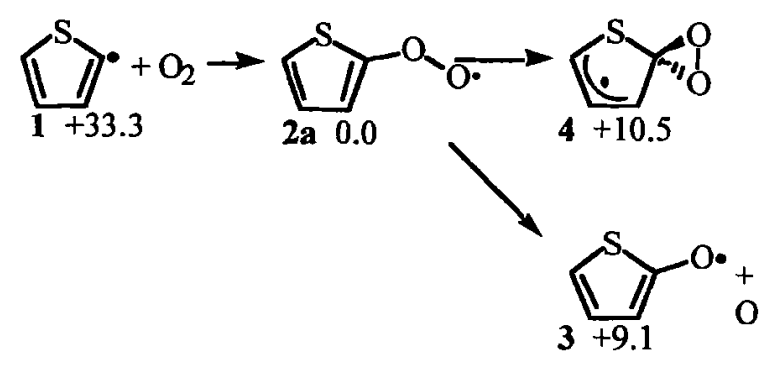

(b)

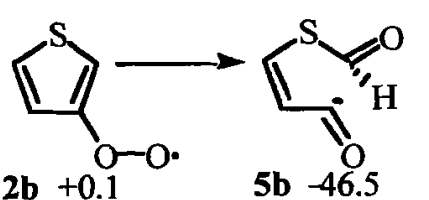

$2 b+0.1$

5b -46.5
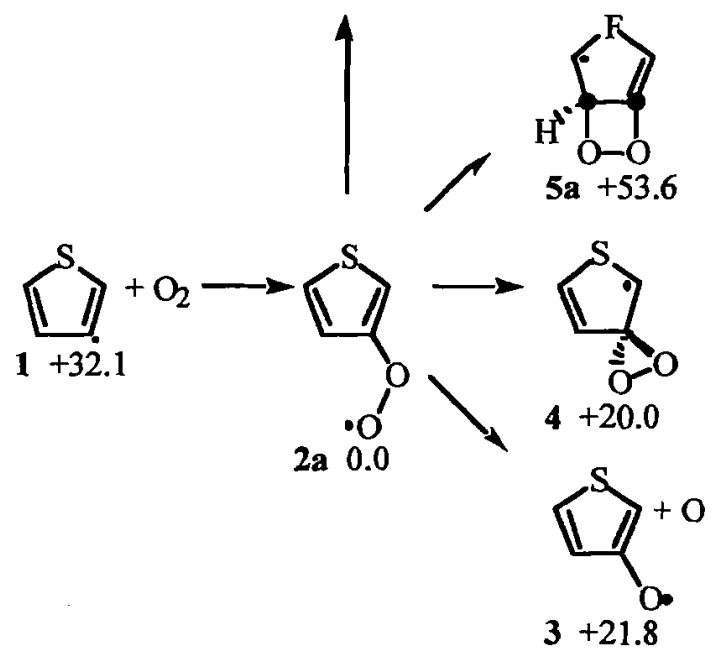

Figure 3.4.7. Mechanisms for the (a) thiophen-2-yl $+\mathrm{O}_{2}$ and (b) thiophen-3-yl $+\mathrm{O}_{2}$ reactions showing Gibbs free energies of each intermediate at $298 \mathrm{~K}$ (relative to the corresponding 2a) at the B3LYP/6-311+G**//B3LYP/6-31G* level. 


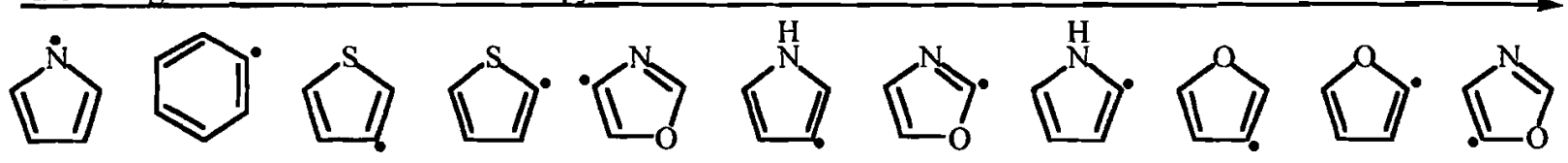

Increasing preference for $\mathrm{O}$ loss over $\mathrm{O}_{2}$ loss from the arylperoxy radical precursor<smiles></smiles>

Increasing preference for $\mathrm{O}$ loss over dioxiranyl ring formation from the arylperoxy radical precursor<smiles></smiles>

Decreasing preference for $\mathrm{O}_{2}$ loss over dioxiranyl ring formation from the arylperoxy radical precursor<smiles>c1ccc(-n2cccc2)cc1</smiles>

Figure 3.4.8. Observed trends of 5-membered heterocyclic radical oxidation and further rearrangements as compared to the related C-H BDE in the parent aromatic ring. ${ }^{2}$ See ref. 24.

In general, the preference for the loss of $\mathrm{O}$ atom over the loss of $\mathrm{O}_{2}$ from the 5-membered arylperoxy radical precursor increases for arylperoxy radicals derived from the 2-position over the 3-position, and within these subsets, the preference increases with respect to increasing $\mathrm{C}-\mathrm{H}$ BDE. The only 5-membered arylperoxy radical in this study that prefers $\mathrm{O}_{2}$ loss over $\mathrm{O}$ loss at all temperatures is pyrrol-1-ylperoxy radical (2a) (derived from the $\mathrm{N}$-centered radical). This is due to the unique stability of the pyrrol-1-yl radical (Figure 3.4.6), as this radical, like 
cyclopentadienyl radical, has the unpaired electron in the $\pi$-system - for a total of 5 electrons in the $\pi$-system. ${ }^{24,35,35}$ Most carbon-centered aryl radicals have the unpaired electron in the $\sigma$ system. $^{24}$

The same trend is seen for the relative stability of dioxiranylaryl radicals (4) as compared to aryloxy radicals (3). At 298K, all peroxy radicals derived from the 2-position favor O-atom loss over isomerization to the dioxiranyl product, except for 5-oxazolylperoxy radical. By $500 \mathrm{~K}$, the aryloxy radical intermediate is more stable than every corresponding dioxiranyl radical intermediate except in the case of the pyrrol-1-ylperoxy radical.

Finally, dioxetanyl radical products resulting from the rearrangement of pyrrolylperoxy and thiophenylperoxy radicals have been explored (Figures 3.4.6 and 3.4.7). Only three bicyclic structures were converged to be stable minima using the B3LYP method: pyrrol-1-yl-5a, thiophen-2-yl-5a and thiophen-3-yl-5a. All of the other structures exhibited $\mathrm{O}-\mathrm{O}$ bond scission, a $\mathrm{C}-\mathrm{C}$ bond cleavage, or a combination of both. It is doubtful that transition state structures can be found that directly link the parent peroxy intermediate to either pyrrol-2-yl-5b or pyrrol-3-yl5a dioxetanyl radicals based on our previously noted observations in the furanyl and oxazolyl potential energy surfaces. With regard to the dioxetanyl radicals derived from the thiophenyl system, it is highly likely that direct linkage between thiophenylperoxy radical and each dioxetanyl radical may exist due to the increased polarizability of sulfur as compared to oxygen.

\subsubsection{Conclusions}

We have explored the potential energy surfaces, including transition states, for the reaction and subsequent rearrangement of $\mathrm{O}_{2}$ addition to furanyl and oxazolyl radicals. Thermodynamic comparisons have been presented for these systems with the thiophenyl and pyrrolyl radical systems. A range of temperatures relevant to both atmospheric and combustion processes have been explored for all of these reaction mechanisms. 
Throughout the 5-membered ring heterocycles, the formation of the aryloxy intermediate is generally the most favored intermediate evolving from the arylperoxy radical. This preference is greater than that observed in the phenylperoxy radical ${ }^{14,16}$ or other 6-membered ring heterocycles. ${ }^{15}$ The thermodynamic stability and activation barriers for formation of the dioxiranyl intermediates are comparable to the aryloxy intermediates at low temperatures. However, the dioxetanyl radical intermediates are much less favored. If the dioxetanyl radicals demonstrate more stability than their aryloxy counterparts, they suffer kinetically with higher activation barriers or the lack of a direct pathway (i.e. no transition state) for formation from the initial arylperoxy radical intermediate.

\section{References for Section 3.4:}

${ }_{35}$ (a) Smith, K. L.; Smoot, L. D.; Fletcher, T. H.; Pugmire, R. J. The Structure and Reaction Processes of Coal; Plenum Press: New York, 1994. (b) Meyers, R. A. Coal Structure; Academic Press: New York, 1982.

${ }^{35}$ Brezinsky, K. Prog. Energy Combust. Sci. 1986, 12, 1-24.

${ }^{35}$ Bittker, D. A. Combust. Sci. and Tech. 1991, 79, 49-72.

${ }^{35}$ Colussi, A. J.; Zabel, F.; Benson, S. W. Int. J. Chem. Kinet. 1977, 9, 161-178.

${ }^{35}$ Zhang, H.-Y.; McKinnon, J. T. Combust. Sci. and Tech. 1995, 107, 261-300.

${ }^{35}$ Chai, Y.; Pfefferle, L. D. Fuel 1998, 77, 313-320.

${ }^{35}$ Morris, V. R.; Bhatia, S. C.; Stelson, A. W.; Hall, J. H. Energy \& Fuels 1991, 5, 126-133.

${ }^{35}$ Fulle, D.; Dib, A.; Kiefer, J. H.; Zhang, Q.; Yao, J.; Kern, R. D. J. Phys. Chem. A 1998, 102, 7480-7486.

${ }^{35}$ Organ, P. P.; Mackie, J. C. J. Chem. Soc. Faraday Trans. 1991, 87, 815-23.

${ }^{35}$ Lifshitz, A.; Bidani, M.; Bidani, S. J.Phys. Chem. 1986, 90, 5373-5377.

${ }^{35}$ Grela, M. A.; Amorebieta, V. T.; Colussi, A. J. J. Phys. Chem. 1985, 89, 38-41.

${ }^{35}$ Lifshitz, A.; Tamburu, C.; Suslensky, A. J. Phys. Chem. 1989, 93, 5802-5808. 
${ }^{35}$ Sendt, K.; Bacskay, G. B.; Mackie, J. C. J. Phys. Chem. A 2000, 104, 1861-1875.

${ }^{35}$ Fadden, M. J.; Barckholtz, C.; Hadad, C. M. J. Phys. Chem. A 2000, 104, 3004-3011.

${ }^{35}$ Fadden, M. J.; Hadad, C. M. J. Phys. Chem. A 2000, in press.

${ }^{35}$ Barckholtz, C.; Fadden, M. J.; Hadad, C. M. J. Phys. Chem. A 1999, 103, 8108-8117.

${ }^{35}$ Frisch, M. J.; Trucks, G. W.; Schlegel, H.B.; Gill, P. M. W.; Johnson, B. G.; Robb, M. A.; Cheeseman, J. R.; Keith, T.; Petersson, G. A.; Montgomery, J. A.; Raghavachari, K.; Al-Laham, M. A.; Zakrzewski, V. G.; Ortiz, J. V.; Foresman, J. B.; Peng, C. Y.; Ayala, P. Y.; Chen, W.; Wong, M. W.; Andres, J. L.; Replogle, E. S.; Gomperts, R.; Martin, R. L.; Fox, D. J.; Binkley, J. S.; Defrees, D. J.; Baker, J.; Stewart, J. J. P.; Head-Gordon, M.; Gonzalez, C.; Pople, J. A. Gaussian 94, Revision D.3, Gaussian, Inc.; Pittsburgh, PA, 1995.

${ }^{35}$ Frisch, M. J.; Trucks, G. W.; Schlegel, H. B.; Scuseria, G. E.; Robb, M. A.; Cheeseman, J. R.; Zakrzewski, V. G.; Montgomery, J. A., Jr.; Stratmann, R. E.; Burant, J. C.; Dapprich, S.; Millam, J. M.; Daniels, A. D.; Kudin, K. N.; Strain, M. C.; Farkas, O.; Tomasi, J.; Barone, V.; Cossi, M.; Cammi, R.; Mennucci, B.; Pomelli, C.; Adamo, C.; Clifford, S.; Ochterski, J.; Petersson, G. A.; Ayala, P. Y.; Cui, Q.; Morokuma, K.; Malick, D. K.; Rabuck, A. D.; Raghavachari, K.; Foresman, J. B.; Cioslowski, J.; Ortiz, J. V.; Stefanov, B. B.; Liu, G.; Liashenko, A.; Piskorz, P.; Komaromi, I.; Gomperts, R.; Martin, R. L.; Fox, D. J.; Keith, T.; Al-Laham, M. A.; Peng, C. Y.; Nanayakkara, A.; Gonzalez, C.; Challacombe, M.; Gill, P. M. W.; Johnson, B.; Chen, W.; Wong, M. W.; Andres, J. L.; Gonzalez, C.; Head-Gordon, M.; Replogle, E. S.; Pople, J. A. Gaussian 98, Revision A.7, Gaussian, Inc.; Pittsburgh, PA, 1998.

${ }^{35}$ (a) Labanowski, J. W.; Andzelm, J. Density Functional Methods in Chemistry; Springer: New York, 1991. (b) Parr, R. G.; Yang, W. Density Functional Theory in Atoms and Molecules;

Oxford University Press: New York, 1989.

${ }^{35}$ Becke, A. D. Phys. Rev. A 1988, 38, 3098-3100.

${ }^{35}$ Lee, C.; Yang, W.; Parr, R. G. Phys. Rev. B. 1988, 37, 785-789.

${ }^{35}$ Becke, A. D. J. Chem. Phys. 1993, 98, 1372.

${ }^{35}$ Hehre, W. J.; Radom, L.; Schleyer, P. v. R.; Pople, J. A. Ab Initio Molecular Orbital Theory; John Wiley \& Sons: New York, 1986.

${ }^{35}$ Barckholtz, C.; Barckholtz, T. A.; Hadad, C. M. J. Am. Chem. Soc. 1999, 121, 491-500.

${ }^{35}$ (a) Cioslowski, J.; Liu, G.; Martinov, M.; Piskorz, P.; Moncrieff, D. J. Am. Chem. Soc. 1996, 118, 5261-5264. (b) Cioslowski, J.; Liu, G.; Moncrieff, D. J. Org. Chem. 1996, 61, 4111-4114.

${ }^{35}$ Bauschlicher, C. W., Jr.; Langhoff, S. R. Mol. Phys. 1999, 96, 471. 
${ }^{35}$ Stanton, J. F.; Gauss, J.; Watts, J. D.; Lauderdale, W. J.; Bartlett, R. J. Int. J. Quant. Chem. 1992, S26, 879.

${ }^{35}$ Scott, A. P.; Radom, L. J. Phys. Chem. 1996, 100, 16502-16513.

${ }^{35}$ (a) Gonzalez, C.; Schlegel, H. B. J. Chem. Phys. 1989, 90, 2154. (b) Gonzalez, C.; Schlegel, H. B. J. Phys. Chem. 1990, 94, 5523.

${ }^{35}$ Rablen, P. R., Thermo94, Yale University, 1994.

${ }^{35}$ Chase, M. W., Jr. NIST-JANAF Thermochemical Tables; Washington, DC: American Chemical Society; New York: American Institute of Physics for the National Institute of Standards and Technology, 1998.

${ }^{35}$ Bredt, J.; Thouet, H.; Schmitz, J. Liebigs Ann. Chem. 1924, 437, 1.

${ }^{35}$ Blank, D. A.; North, S. W.; Lee, Y. T. Chem. Phys. 1994, 187, 35-47.

${ }^{35}$ Backsay, G. B.; Martoprawiro, M.; Mackie, J. C. Chem. Phys. Lett. 1998, 290, 391-398. 


\section{Chapter 4: Mesoscale Structural Models}

This chapter describes two new approaches to the quantitative description of order development in chars and other carbon materials. The first approach uses thermodynamic tools to examine the equilibrium order. It is directly applicable to highly fluid carbonizing systems (e.g. pitches), where molecular mobility is sufficient to achieve the equilibrium state. In addition, knowledge of the equilibrium state is an important beginning in the understanding of less fluid systems (most coals), where rearrangement kinetics also play a role. The first section describes such a thermodynamic approach that builds on early work done under an NSF CAREER project and results in the most advanced model of mesophase formation in existence today.

The second section describes a very new approach that accounts for molecular mobility limitations that influence the structure of coal chars. This work was done entirely for the present project and was accomplished with important collaborative contributions from Dr. Alan Kerstein at Sandia National Laboratories.

\section{Section 4.1: Thermodynamic Modelling of Order / Disorder Transitions in the Synthesis of Carbon Materials: Effect of Low-MW Solvents}

Near the end of the project, the task on modeling carbon nanostructure formation was significantly extended to include effects on low molecular weight solvents and a more robust theory of non-ideal mixing effects in high-molecular-weight polyaromatic mixtures. This work was done in collaboration with the group of Prof. Mark Thies at Clemson University. This third generation model (extending our two previous model formulations $[6,2]$ is described in some detail below.

Long-range crystalline order in carbon materials often arises through the formation of carbonaceous mesophase, the naturally occurring discotic liquid crystal composed of highmolecular weight polycyclic aromatic molecules. There have been two theories to describe the formation of mesophase: (1) high-MW pitch fractions have limited solubility in the lower MW fractions, leading to precipitation of the high-MW mesophase, and (2) the free energy of the highMW pitch molecules is reduced by a concerted molecular orientation. These two theories are based on different chemical principles and are described by different classes of models. The second theory is the basis for the first quantitative model of mesophase formation by Shishido et al. [1] based on liquid crystal theory .

Recently we have proposed a model of mesophase formation [2] that unified these two approaches by integrating a submodel for non-ideal mixing with a submodel derived from the statistical molecular theories of the liquid crystalline state [3]. The model describes mesophase formation in a variety of pitches, but does not rigorously consider the effect of low-molecular weight solvents.

Other work has treated solvent extraction of pitches under supercritical conditions using the Statistical Associating Fluid Theory (SAFT) approach [4,5], but does not treat molecular orientation as needed to identify mesophase. The present report combines the SAFT approach with the statistical liquid crystal theory to develop a new model capable of describing both solvent processing and mesophase formation with a single thermodynamic formulation. 


\section{Model Development}

The current model is based on a free energy expansion of the form:

$$
\mathrm{a}^{\text {res }}=\mathrm{a}^{\mathrm{SAFT}}+\mathrm{a}^{\text {orient }}
$$

where $\mathrm{a}^{\text {res }}$ is the residual free energy. The SAFT approach describes non-ideal mixing in complex liquids by modeling the molecules as chains of Lennard-Jones spheres bonded by covalent linkages. The individual segments on a chain will interact with other chains through association "bonds," leading to the expansion:

$$
\mathrm{a}^{\mathrm{SAFT}}=\mathrm{a}^{\mathrm{seg}}+\mathrm{a}^{\text {chain }}+\mathrm{a}^{\text {assoc }}
$$

The orientational term in Eq. (1) is modeled using the statistical theory of liquid crystal mixtures of Luckhurst and coworkers [3], following Shishido [1] and our previous work [2]. The primary relation is:

$$
\begin{array}{r}
a^{\text {orient }=-\frac{N}{2} \sum_{1, j} x_{i} x_{j} e_{i j} \bar{P}_{i} \bar{P}_{j}} \\
-R T \sum_{i} x_{i} \ln Z_{i}
\end{array}
$$

where $\overline{\mathrm{P}}_{\mathrm{i}}$ are the single component order parameters. Previous publications present the full development of the SAFT equation [4,5] and the statistical LC theory [2,3]. A number of auxiliary relations are necessary to complete the model, which in its final form has 4 parameters: " $A$ " and " $B$ " which describe isotropic molecular interactions between the pitch molecules and the solvent, " $\mathrm{C}$ " which describes the isotropic molecular interactions between pitch molecules of differing molecular weight, and " $\alpha$ " which describes the effect of molecular weight on the "clearing temperature" a measure of the orientational potential or liquid crystal forming tendency of a discotic.

\section{Model Validation and Application}

The new model was fit to previously published data of Thies and coworkers $[4,5]$ and the results summarized in Figs. 1, 2, and Table 1. The model was able to fit the extraction compositions (Fig. 1) and the mesophase contents (Fig. 2) using a common optimized parameter set (Table 1).

Note that in the previous work by Thies and coworkers on extraction, the " $\mathrm{C}$ " parameter was set to zero, since pitch/pitch interactions were insignificant compared to solvent/pitch interactions. In the present work in which we also attempt to predict mesophase formation, and therefore find that the "C" parameter must be non-zero. Because the mesophase contents in Fig. 2 were determined in the absence of solvent, pitch/pitch non-ideal mixing become important for prediction of mesophase occurrence. As concluded in the previous paper in this series [2], mesophase forms (in Fig. 2) due to the combined effect of pitch/pitch non-ideal mixing (embodied here by parameter " $\mathrm{C}$ ") and due to orientation free energy (embodied here by parameter " $\alpha$ "). 


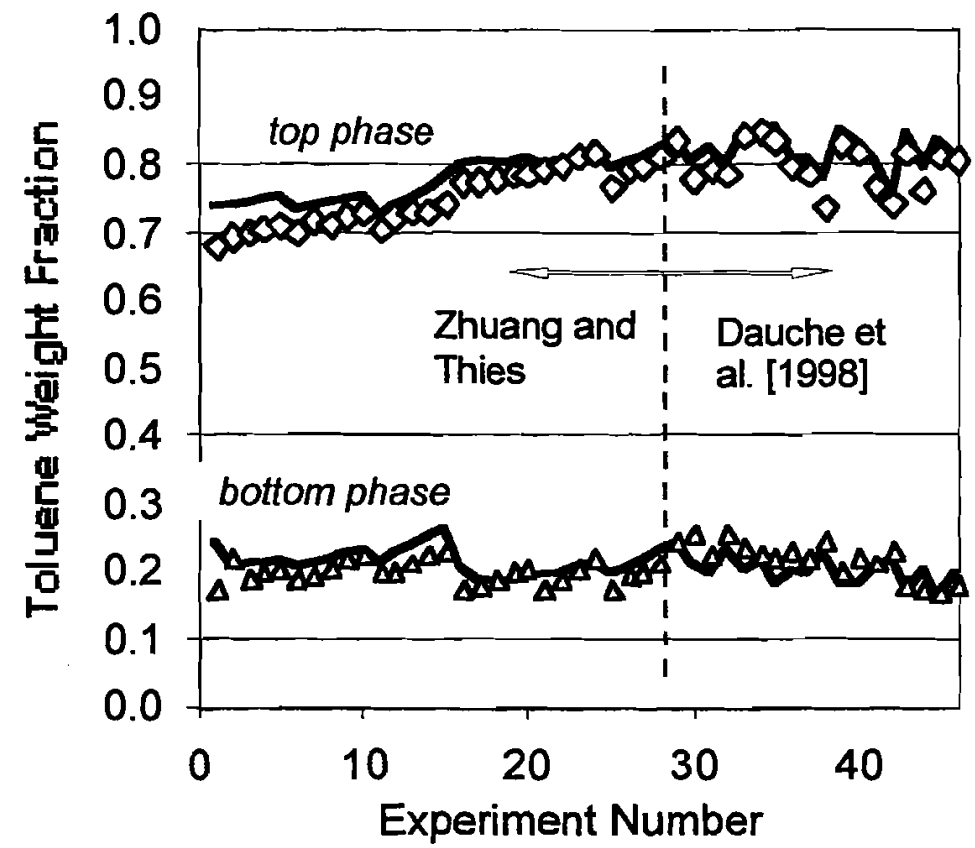

Figure 1. Composition of top and bottom phases during pitch extraction with toluene under a variety of experimental conditions. Symbols are experimental data; lines show predictions of the current model.

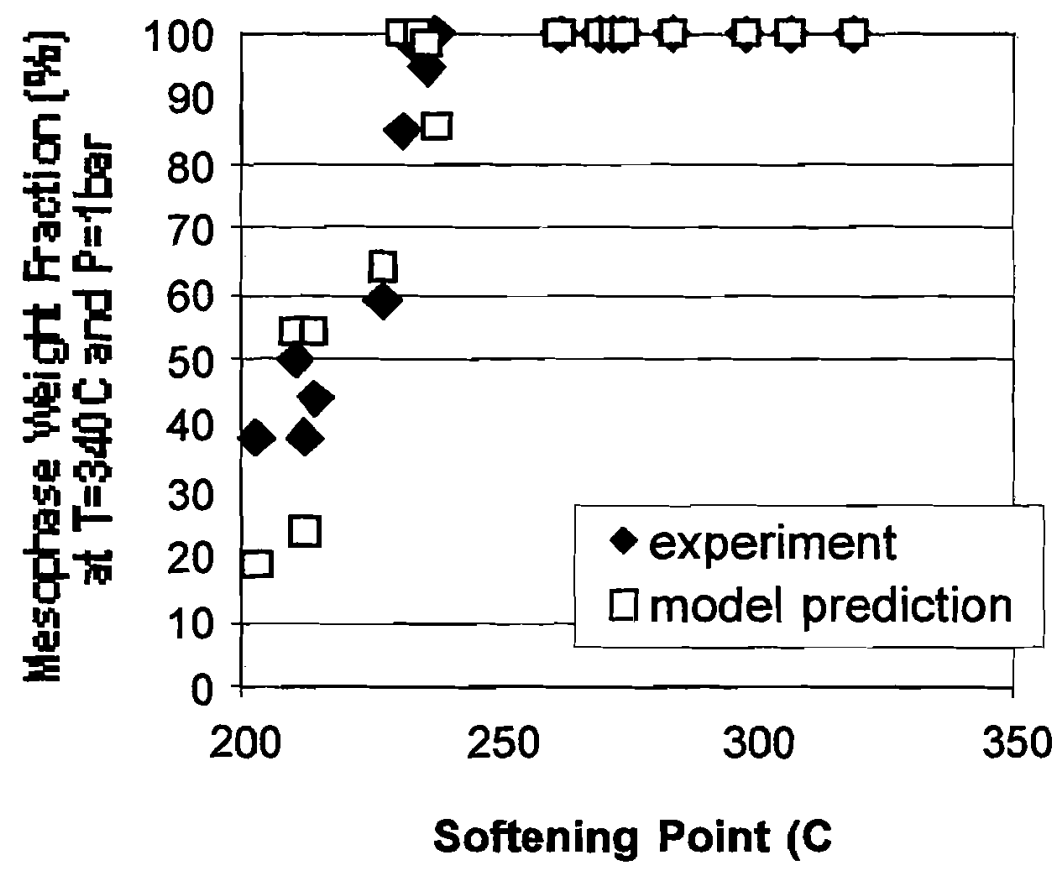

Figure 2. Mesophase content in bottom phase from pitch extraction with toluene under a variety of experimental conditions. Experimental samples are ordered based on the measured softening point of the bottom phase. 
Table 1 Model parameters

used for predictions in Figs. 1 and 2.

\begin{tabular}{|c|c|c|}
\hline Parameters & Previous & $\begin{array}{l}\text { Present } \\
\text { model }\end{array}$ \\
\hline $\mathrm{A}(\mathrm{mol} / \mathrm{gm})$ & $5.3 \cdot 10^{-3}$ & $6.6 \cdot 10^{-3}$ \\
\hline & $3.0 \cdot 10^{-2}$ & $5.5 \cdot 10^{-2}$ \\
\hline $\mathrm{C}(\mathrm{mol} / \mathrm{gm})$ & 0 & $5.0 \cdot 10^{-6}$ \\
\hline$\alpha(\mathrm{mol}-\mathrm{K} / \mathrm{gm})$ & N/A & 0.38 \\
\hline
\end{tabular}

Figure 3 shows one example of a parameter study carried out with the validated model. Many other parameter studies are possible, and we believe this model will have applications in the optimization of industrial pitch processing conditions.

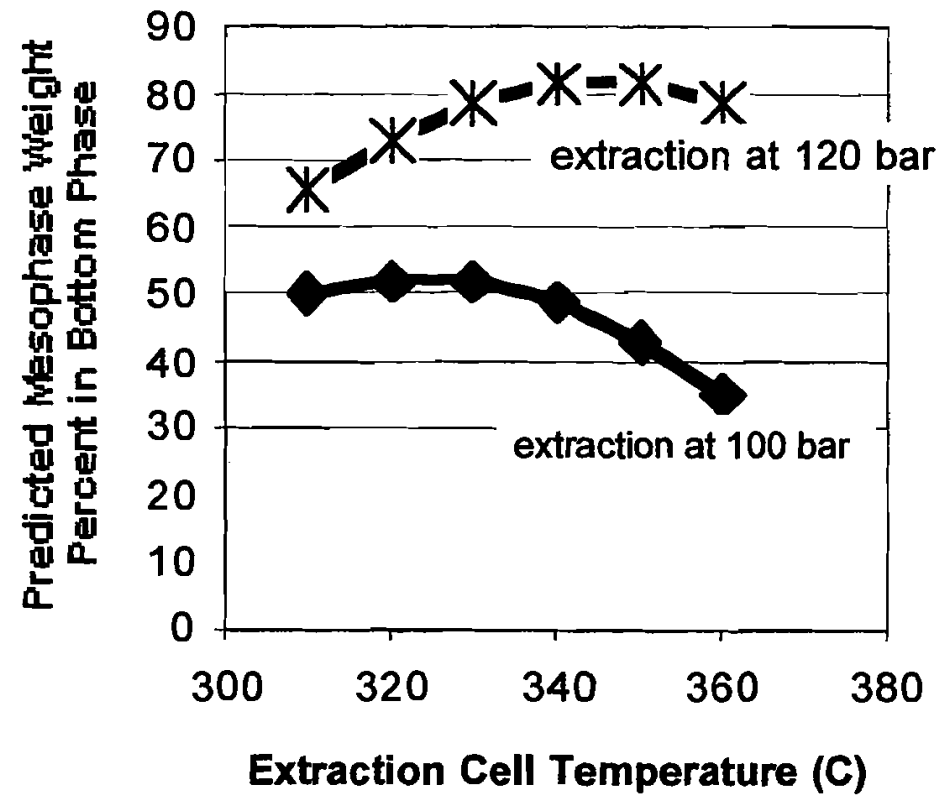

Figure 3. Example parameter study with validated model. Result shows the presence of an optimal extraction temperature to maximize the mesophase mass fraction in bottom phase.

\section{References for Section 4.1}

1. Shishido M., Inomata H., Arai K. and Saito S., Carbon, 1997, 35, 797.

2. Hu, Y., Hurt, R.H., "Thermodynamics of Carbonaceous Mesophase II: General Theory for Nonideal Solutions," Carbon 39 887-896 (2001).

3. Humphries RL, Luckhurst GR. Proc R Soc Lond A 1976;352:41.

4. Zhuang MS, and Thies MC. Energy and Fuels, 2000;14:70.

5. Dauche F M, Bolanos G, Blasig A, Thies MC, Carbon 1998;36(7-8):953-961.

6. Hurt, R.H., Hu, Y. "Thermodynamics of Carbonaceous Mesophase," Carbon 37 281-292 (1999). 
Section 4.2: Kinetics of Orientational Order / disorder Transitions and their Application to Carbonization of Coals and Other Organic Materials

Liquid crystals are shear-deformable fluids composed of non-equiaxed molecules that exhibit orientational order but no long-range positional order. Isotropic liquids transform to liquid crystalline phases upon a decrease in temperature (thermotropic transition) or an increase in the concentration of the non-equiaxed molecules in solution (lyotropic transition). The liquid crystalline phase is favored by high aspect ratio, $L / D$, and high number density of the liquid crystal forming molecules, or "mesogens". Both the unique physics of liquid crystalline phases and their important commercial applications have led to an extensive literature on their structure [De Gennes and Prost, 1993], electromechanical properties [Chandresekhar, 1992; De Gennes and Prost, 1993], rheology [Chandresekhar, 1992], the thermodynamic properties of their phase transitions[Barrall, 1979; Maier and Saupe, Onsager, 1949], and their computer simulation by Monte Carlo methods and molecular dynamics [Lee et al., 1992; Gay and Bern, 1981; Bates and Luckhurst, 1996].

This section considers the kinetics of the orientational order / disorder transitions with special application to those systems in which molecular mobility is limited. Potential applications include non-equilibrium cooling of isotropic liquid phases, and lyotropic transitions in which the mesogens are dissolved or suspended in viscous solvents. An application of particular interest is the synthesis of carbon materials, so-called carbonization processes, involving condensed phase organic starting materials.

Many carbon properties depend on nanostructure - the spatial arrangement of the graphene layers that are the basic building blocks of the material [Oberlin, 1990]. Carbon materials formed from condensed phase organic precursors can be grouped into two categories: anisotropic carbons, in which the graphene layers possess long range orientational order, and isotropic carbons, in which the graphene layers exhibit short range order only [Marsh and Walker, 1979] (see Fig. 1). For many organic precursors poor in oxygen and stable sulfur, nanostructural ordering occurs in the molten stage of carbonization resulting in a discotic liquid crystalline intermediate known as carbonaceous mesophase [Marsh and Walker, 1979; Gasparoux, 1979]. The solidification of carbonaceous mesophase by chemical crosslinking upon further heat treatment results in the first category of carbon materials - those with long-range orientational order and visible optical anisotropy (Fig. 1c,d). Carbonaceous mesophase is believed to be a discotic liquid crystal composed of nearly planar but incompletely condensed oligomers of small aromatic clusters [Lewis 1982]. This carbonization process bears some similarity to the formation of highly ordered polymer networks by thermal crosslinking of liquid crystalline monomer precursors [Clough et al., 1976].

Carbonaceous mesophase can be formed through decreases in temperature [Lewis, 1978], or through solvent extraction which removes low molecular weight material and increases the concentration of the high molecular weight mesogens [Riggs and Diefendorf, 1980]. Carbonaceous mesophase is thus seen to have a duel thermotropic and lyotropic nature. In practice, however, carbonaceous mesophase is most commonly formed through chemical growth processes involving dehydrogenative condensation reactions between small polyaromatic clusters, producing progressively larger oligomers with near-planar geometries. In an isothermal carbonization process, these chemical events lead to increases in mean molecular weight, which increases the effective 
aspect ratio of the discotic oligomers (as their thickness is nearly constant) and increases their liquid crystal forming tendency. At $\sim 1000$ amu a critical aspect ratio is reached and the molecules undergo a phase transition to the ordered discotic liquid crystal. Accompanying this increase in mesogen size is typically an increase in mesogen concentration, as low-molecular-weight, nonmesogenic compounds are lost by vaporization and chemical coalescence. There have been several attempts to model the formation of carbonaceous mesophase and the properties of its phase transitions using liquid crystal theory [Riggs and Diefendorf, 1980, Shishido et al., 1997, Hurt and $\mathrm{Hu}, 1998]$.

Isotropic carbons, comprising the second category of carbon materials, possess only short-range order, and are often formed under conditions in which the mobility of the constituent molecules is too low to allow the concerted rotational rearrangements necessary for liquid crystal formation (see Fig. 1a,b). Indeed, many of the organic precursors that form isotropic carbons do not visibly soften or fuse upon heating - they undergo carbonization in the solid state. If solidification occurs in the isotropic state, it is not possible to introduce long range orientational order by further heat treatment. Instead, only local rearrangements occur, leading to the development of somewhat larger crystallites with nearly random orientation in a solid that is isotropic at long length scales. The development of long range order in the early stages of carbonization is thus key to the ultimate production of highly ordered graphites - this is a central concept in carbon science and underscores the importance of understanding the order transition in the early stages of carbonization [Marsh and Walker, 1979].

The goal of this report section is to explore the kinetics of orientational order / disorder transitions with emphasis on highly hindered systems using simple numerical simulations. It will be demonstrated that a numerical "toy" model embodying the essential competition between growth rate and mobility in anisometric objects is capable of reproducing a number of key features of carbonization processes for a variety of organic precursors.

\section{The Numerical Model}

A simple theory was sought to describe the structural transformations occurring during the growth of anisometric objects under conditions of limited, but variable mobility. Numerical simulations were therefore conducted in which canonical anisometric objects (hard lines) grow, rotate, and translate in a two dimensional continuum. The primary advantage of the idealized geometry and reduced dimensionality is simplicity; the simulations will be shown later to be fully defined by only two dimensionless parameters, one of which has primary significance. The simulations proceed as follows. Points are chosen at random in a two-dimensional simulation box of unit length and allowed to grow into lines at a fixed rate, $G$, with growth occurring at both ends. The lines are also allowed to translate and rotate with mean linear and angular velocities, $V$ and $R$, respectively. Random numbers are generated at each time step to determine the actual change in position and orientation for a proposed motion event. Overlap between the lines is forbidden, and thus both growth and motion are carried out in a given time step only if they do not lead to overlap. In the specific algorithm used, small growth steps alternate with small translation/rotation steps until the structure freezes into a gel and no more motion or growth is possible. The simulation algorithm is listed in Table 1 and example structural time sequences are shown in Figs. 1 and 2 below. 


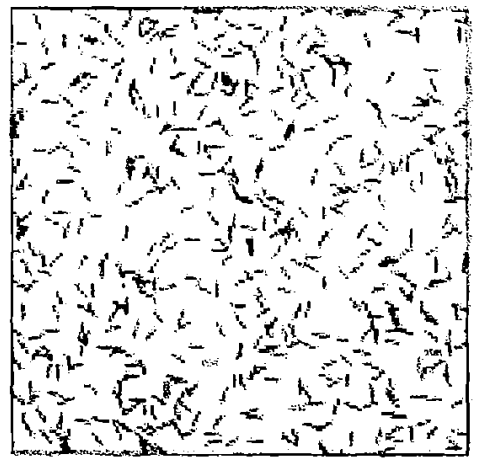

$T^{*}=3,600$

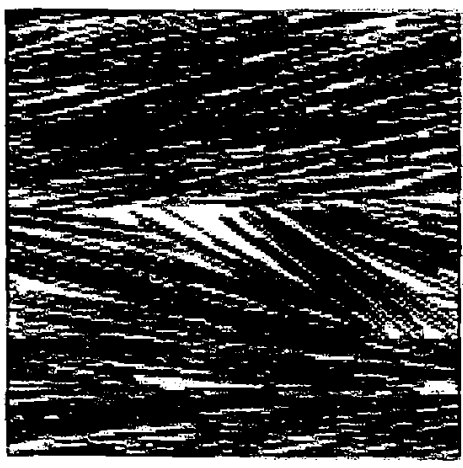

$\mathrm{T}^{*}=32,000$

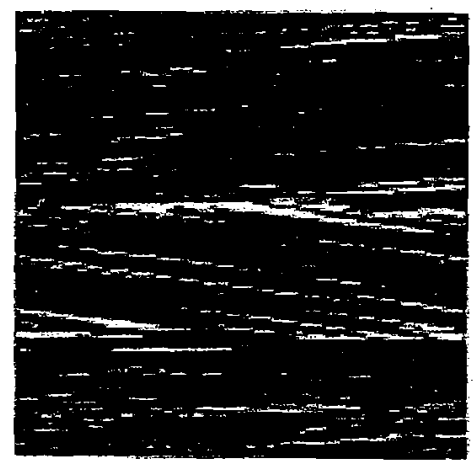

$\mathrm{T}^{*}=65,000$

Figure 1. Hard-line simulations at high mobility/growth ratio: time sequence showing the structural evolution predicted for $M^{*}=2,200$.

At early times, the lines interact infrequently, their rotations are largely unhindered, and disorder prevails (see first panel in Fig. 1). As growth proceeds the lines begin to interact frequently, and adopt a variety of ordered or disordered arrangements depending on the simulation parameters. Figure 2 shows the development of short range orientational order reminiscent of distinct crystallites in an isotropic solid. Figure 2 shows the development of order with a length scale equal to or greater than the size of the simulation box. As growth proceeds further, the structure gels in a state where each line has two points of contact with neighbors and no further growth is possible. A number of parameters must be specified in order to carry out a simulation, but the dimensional analysis presented subsequently shows that, for sufficiently small step size, the model results depend on only two dimensionless parameters, one describing the ratio of orientational mobility to growth and one of lesser importance describing the ratio of translational to orientational mobility. The following sections present the theory necessary to quantify order in the line structures, and to give structure to the set of independent variables that are the input to the simulations.

\section{The nematic order parameter}

The extent of orientational order in a set of lines whose two ends are indistinguishable is characterized by the nematic order parameter [De Gennes and Prost, 1992], which in two dimensions takes the form:

$$
S=2<\cos ^{2} \theta_{i}>-1
$$

where $\theta_{i}$ is the angle between the vector of preferred orientation (the "director"), and the orientational unit vector of layer $i$, and the angled brackets denote an average over all objects. The director itself can be found from the set of unit vectors defining the orientation of the lines by solution of the eigenvalue problem [Hicklas et al., 1994]:

$$
Q \overrightarrow{\mathrm{d}}=\lambda \overrightarrow{\mathrm{d}}
$$

where $d$ is the director and $Q$ is the order tensor: 


$$
Q \equiv(1 / N) \Sigma\left[\begin{array}{cc}
x_{i} x_{i}-1 / 2 & x_{i} y_{i} \\
y_{i} x_{i} & y_{i} y_{i}-1 / 2
\end{array}\right]
$$

where $x_{i}$ and $y_{i}$ are the endpoints of the unit directional vectors for object $i$. For an infinite set of lines, $S$ has a value of zero for random lines (complete disorder) and a value of one for a set of lines all parallel to the director (perfect order). This definition is inadequate for small sample sets, as even sets of randomly chosen lines have mean order parameters systematically greater than zero. In particular, for sets of random line pairs, $(n=2)$, the expected value of the order parameter is not zero but $2 / \pi$ [Shim et al., 1998]. The effect arises when the director is not known a priori, but must be determined from the given set of lines. The choice of the director from the small set of lines biases the result in favor of apparent order, an effect that decreases rapidly as sample size increases. The function that represents the expected value of the order parameter for $n$ random lines, $f_{\text {ran }}(n)$, has been determined numerically and has been used to define a new order parameter, valid for small sample sets [Shim et al., 1998] as:

$$
S(n)=\frac{S-f_{\text {ran }}(n)}{1-f_{\text {ran }}(n)}
$$

This definition is particularly useful when calculating length-dependent order parameters, which for small length scales necessarily involve small sample sizes. The length-dependent order parameter is a useful tool for describing short-range polycrystalline order such as is found in the frozen state in Figure 2.

\section{Dimensional analysis}

Consider the simulation algorithm in Table 1, which describes the dynamic arrangement of growing lines with a number density $\rho$ and mean rotational velocity $R$, and translational velocity $V$. The lines grow at a rate $G$ and the final structure is characterized by an order parameter $S$. The physical problem is defined by specification of the first four parameters with the fifth, the order parameter, representing the result.

The appearance of five parameters involving two fundamental units (length and time) indicates the necessity for three dimensionless groups. We choose the order parameter itself as one, as well as a dimensionless mobility / growth ratio, defined as

$$
M^{*}=R /\left(G \rho^{l / 2}\right)
$$

representing the relative rates of rotation and growth, and a translation / rotation ratio:

$$
V^{*} \equiv V \rho^{I / 2} / R
$$

The problem can now be formulated as:

$$
S_{\text {final }}=f\left(M^{*}, V^{*}\right)
$$

and the simulation results plotted as $S_{\text {final }}$ vs. $M^{*}$ with $V^{*}$ as a parameter. $M^{*}$ is the key parameter representing the competition between growth and orientation, so the initial simulations have been 
done with a constant value of $V^{*}$ (4.5) implying a constant ratio of translational to angular velocity ${ }^{\dagger}$. Varying $V^{*}$ from 1 to 30 (at $\mathrm{M}^{*}=37$ ) had only a small influence on the final structure and order parameters, suggesting that this variable has a secondary importance to $M^{*}$. Additional results include a dimensionless mean layer length, $L^{*} \equiv L_{\text {mean }} \rho^{1 / 2}$, and length-dependent order parameters, $S(r)$, defining the characteristic scale of ordered structures. Finally, the time-dependent results yield intermediate states characterized by $S$ or $S(r)$ as a function of the dimensionless time, $T^{*} \equiv t \cdot R$.

\section{Table 1}

Simulation Algorithm

(Cases with zero initial length and random initial orientation)

1. Define a square simulation box of unit length and place $n$ points (here 500 ) at coordinates $\left(x_{i}, y_{i}\right)$ where $x_{i}, y_{i}$ are random numbers between 0 and 1 .

2. Select a random angle $\theta_{i}$ for each point defining the initial growth direction.

3. Select each line in turn and perform the following steps*:

a) increase its length by $\Delta L$ on both sides

b) if (a) causes interparticle overlap, choose one side only and grow by $\Delta L$

c) if (b) causes interparticle overlap, choose other side only and grow by $\Delta L$

d) generate two random numbers between 0 and $1, r_{1}, r_{2}$, and move the particle to $\left(x_{o}+0.1 \cdot\left(r_{1},-0.5\right), y_{0}+0.1 \cdot\left(r_{2}-0.5\right)\right)$

e) if (d) causes interparticle overlap, do not move

f) generate a random number between 0 and $1, r_{3}$, and rotate through angle $\left(r_{3}-0.5\right) / 2$ radians

g) if (f) causes interparticle overlap, do not rotate

4. Repeat until no more motions or growth events possible (gel point)

* the numerical constants in this algorithm were chosen arbitrarily, but within a range that gives reasonable probability of successful motion $(\sim 0.5)$ during most of the simulation time.

As an example of how to relate the simulation parameters to the dimensionless variables, consider Eq. 5 for $\mathrm{M}^{*}$. $G$ is specified in the simulation as

$$
G=\Delta L / \Delta t
$$

where $\Delta t$ is the simulation time step and $\Delta \mathrm{L}$ the growth increment. The number density is given by:

and the rotation rate:

$$
\rho \equiv N / L^{2} \text { box }
$$

$$
R=\Delta R / \Delta t
$$

where $\Delta R$ is the rotational increment in radians. Combining egs. 5 with 8-10 yields a simple expression for $M^{*}$ for any simulation:

$$
M^{*}=\Delta R /\left[N^{1 / 2} \Delta L / L_{b o x}\right]
$$

Note that if we express the growth increment as a fraction of the box length, that neither the box length nor the time step must be specified in physical units to perform the simulation. For the simulations carried out here, $\Delta R$ is 0.5 radians, $N=500$ and $\Delta L / L_{\text {box }}$ is treated as the independent variable. The results are expressed as $S_{f i n a l} v s . M^{*}$. In a similar way we get $V^{*}=\left[N^{1 / 2}\left(V \Delta t / L_{b o x}\right) / \Delta R\right]$ for the simulations, and since each of these parameters is held constant, $V^{*}=4.5$ in all simulations. 


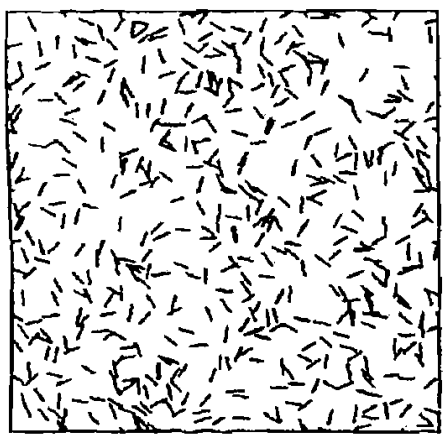

$T^{*}=50$

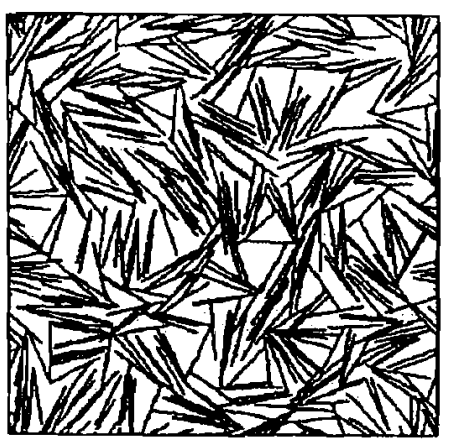

$\mathrm{T}^{*}=210$

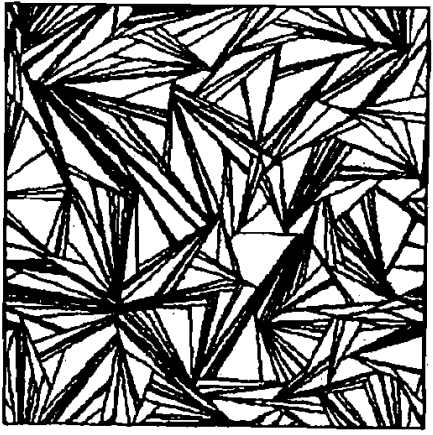

$T^{*}=1,200$

Figure 2. Hard-line simulations at moderate mobility/growth ratio: time sequence showing the structural evolution predicted for $M^{*}=37$. Note the distinct polycrystalline short range order in the frozen state image (dimensionless time, $\mathrm{T}^{*}=1,200$ ).

\section{Discussion of simulation results}

Figures 1 and 2 present time sequences for two values of $M^{*}$, while Figure 3 shows the final frozen states for three values of $M^{*}$. At high line mobility $\left(M^{*}=2.2 \cdot 10^{3}\right)$, mutual avoidance causes the lines to align during the later stages of growth leading to an phase exhibiting order on a length scale comparable to the simulation box. At lower mobility / growth ratio $\left(M^{*}=37\right)$, the alignment is hindered and the lines adopt a final state with short range order only, reminiscent of distinct crystallites with random orientation in isotropic carbon materials. At $M^{*}=0$, corresponding to zero mobility or to infinite growth rate, the simulations produce the random structure seen in the leftmost frozen state in Fig. 3. Overall order parameters, $S\left(L_{b o x}\right)$, for the frozen states are plotted as a function of $M^{*}$ in Fig. 4 (solid line). This figure shows a clear transition from order to disorder in a critical range of the mobility / growth ratio from $10^{2}$ to $10^{4}$.

The evaluation of $S$ at $L_{\text {box }}$ in Fig. 4 is an arbitrary choice dependent on the size of the simulation. The results are presented in Fig. 6 in a more general form as the length scale of ordered structures vs. $M^{*}$. The length scale of order, $L_{o r}$, is derived from plots of length-dependent order parameter, $S(r)$, vs. $r$, the radius of the circular region of interest. $S$ decreases monotonically with $r$ and $L_{o r}$ is defined here as the size of the circular region $(2 r)$ at which $S$ has fallen to 0.2 . The length scale of order is shown in Fig. 5 to rise rapidly in the $\log M^{*}$ range from 0 to 2.

There are several alternate physical interpretations for these simulations. If the lines are regarded as solid needles growing in a fluid continuum, the simulation describes a transition from a sol to a rigid gel allowing no further growth. Because the solid elements are anisometric (much larger in one dimension than the other) the system can develop orientational order prior to the gel point in a manner resembling a lyotropic liquid crystal transition. 

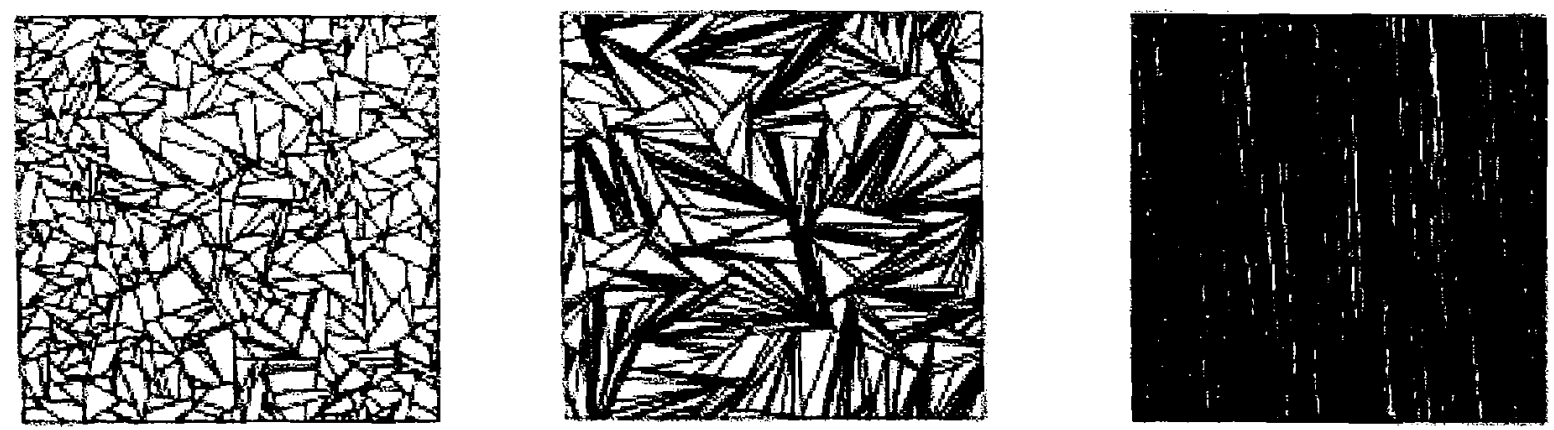

Figure 3. Summary of frozen states in hard-line simulations. As mobility/growth ratio increases, the final structures changes from complete disorder to short range order to a structure in which the ordered length scale is comparable to the size of the simulation box.

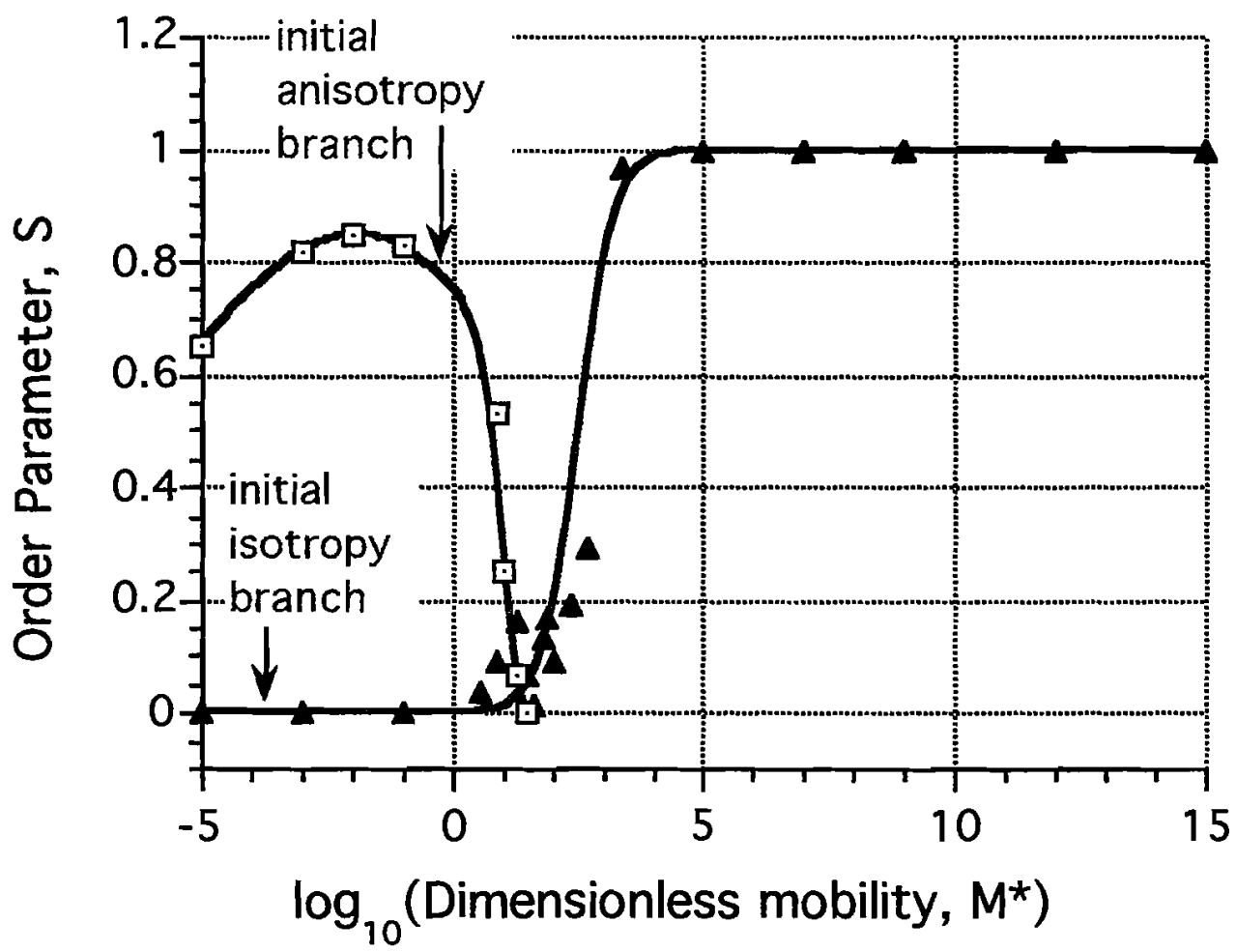

Figure 4. Summary of simulation results: nematic order parameter, $S$, (corresponding to the simulation box length scale) as a function of mobility/growth ratio, $M^{*}$. Simulations begin from initially random states (solid line) representing isotropic parent materials, and initial ordered states (dashed line) representing anisotropic parent materials. Each of these simulations begins with layers of zero length. 


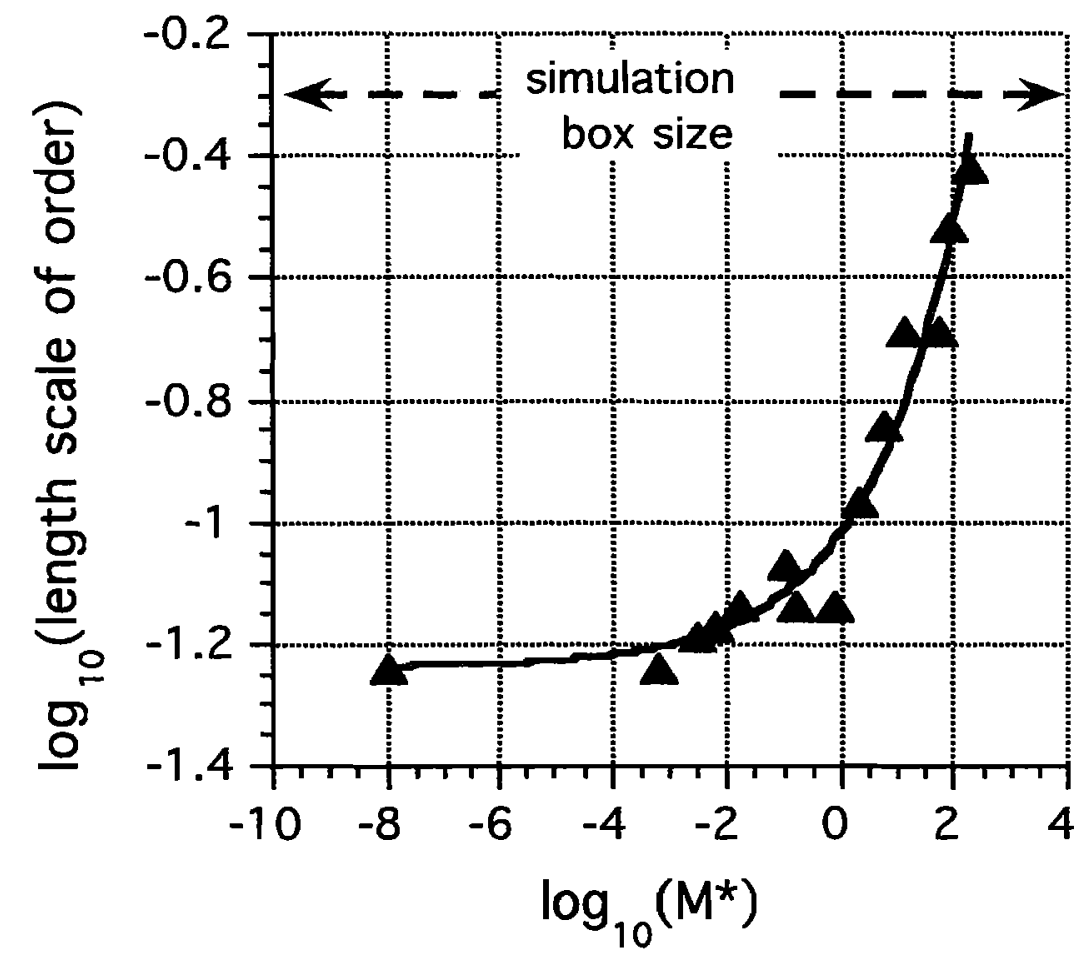

Figure 5 Length scale of order in frozen images as a function of the mobility / growth ratio.

For hard lines of equal length in two dimensions, the state of a chemically frozen (constant $L$ ) system is fully defined by $L(\rho)^{1 / 2}$, where $L$ is the line length and $\rho$ the number density, $N / L_{b o x}{ }^{2}$. This dimensionless parameter is the ratio of line length, $L$, to the characteristic distance between line centers, $n^{-1 / 2}$. When $L(n)^{1 / 2}$ reaches order 1 , the lines interact and adopt an ordered configuration in the equilibrium state. When growth is slow, a near equilibrium state is achieved at each growth step and the liquid crystalline phase is formed at ithreshold value of $L(\rho)^{1 / 2}$, equivalent to a threshold $L$ in these simulations of constant $\rho$. At higher growth rates, the transition is passed quickly and the system reaches the gel point before the equilibrium ordered phase has time to form. The simulations thus describe a kinetically-influenced phase transition.

\section{Application to Carbon Material Synthesis}

The simulations in Figs. 1 - 5 are relevant to carbonization processes, in which nearly planar aromatic ring structures grow at the expense of disordered organic matter while exhibiting a variable mobility. The simulations employ simplified geometry and reduced dimensionality, but embody the essential competition between angular mobility and chemical growth. The layer mobility is dependent on the number and type of crosslinking agents as well as the strength of intermolecular forces (physical and quasichemical) relative to $k T$. Further, the particular initial condition used ( $L=$ $S=0$ ) is appropriate for most carbonization processes, including those in which aromatic ring structures are not prevalent in the organic precursor (e.g. petroleum, aliphatic polymers, cellulose) or are present but much smaller than the planar structures involved in the formation of 
carbonaceous mesophase ${ }^{\dagger}$ (e.g. polystyrene, phenol-formaldehyde resins, lignin, lignites, most bituminous coals). The final structures in Figs. 2 and 3 can be compared to characteristic nanostructures in anisotropic and isotropic carbons, respectively. Carbons referred to as isotropic have no discernible order on length scales visible by optical microscopy, but are revealed by highresolution transmission electron microscopy to possess short range order $(5-100 \mathrm{~nm})$ of a greater or lesser degree depending on the severity of heat treatment conditions [Oberlin, 1989; Rouzaud and Oberlin, 1990]. Figure 5 can reflect the progression from anisotropic to isotropic carbons as the concentration and thermal stability of crosslinking agents increases and layer mobility correspondingly decreases.

The numerical model in its simplest form is thus sufficient to distinguish two important classes of carbon materials: (1) isotropic carbons formed via all-solid-state routes in which molecular mobility is severely limited (e.g. low-rank ${ }^{\dagger \dagger}$ coals, woody and cellulosic materials, and oxygen-rich thermosetting polymers such as phenol-formaldehyde resins), and (2) anisotropic carbons formed from precursors that pass through a mobile, liquid-phase intermediate (high-rank bituminous coals [Marsh, 1973], polyvinyl chloride [Marsh and Walker, 1979], anthracene [Griffin et al., 1991], many petroleum and coal-tar pitches [Greinke, 1994]). There are additional categories of carbon materials that are not described by the model in its simplest form, including many anthracites that do not soften, but form relatively ordered carbons. The next section reviews the experimental observations relevant to these materials and then proposes the minimal modification to the hard line simulations to describe the essential features of these materials.

\section{Some additional features of carbonization processes}

The restrictions of zero initial length and initial order parameter are not appropriate for some carbonization processes, in particular those involving high-rank fossil fuels. Lower-rank coals with dry, ash-free carbon contents below about 85 weight-\% are typically isotropic in their raw state under reflected light [Murchison, 1978]. The combination of time, temperature, and lithostatic (bed) pressure lead to an increasing aromatic cluster size and an increasing degree of orientational order parallel to the bedding plane as rank increases. High-rank bituminous coals ( 85 - $90 \mathrm{wt}-\%$ carbon, dry, ash-free) have aromatic clusters containing an average of $14-20$ aromatic carbons as determined by NMR techniques [Smith et al., 1994], corresponding to mean molecular weights of $300-400 \mathrm{amu}$, including substituent groups. These clusters are only slightly larger than those in lower-rank bituminous and subbituminous coals (10-15 aromatic carbons [Smith et al., 1994]). In each case the mean cluster size is significantly smaller than the critical molecular weight for mesophase formation ( $1000 \mathrm{amu}$ ). The high-rank bituminous coals (dry, ash-free carbon $>85 \%$ )

\footnotetext{
$\dagger_{\text {i.e. the original clusters are much smaller than the critical size for order development, believed to be } \sim 1000 \mathrm{amu}}$ in carbonization [Marsh and Walker, 1979; Lewis, 1982], which corresponds to a disk of $\sim 2 \mathrm{~nm}$ for fully condensed polyaromatic clusters or somewhat larger values for incompletely condensed clusters.

†† Coals are often classified by "rank", a measure of the degree of transformation of the original plant matter by temperature and bed pressure acting over geological time scales. In practice, rank can be defined by any one of a number of coal properties that vary systematically as the organic matter is gradually transformed from a complex, disordered, heteroatom-rich carbonaceous polymer toward the thermodynamic equilibrium state, graphite.
} 
show finite values of bireflectance, an measure of anisotropy computed as the difference between the minimum and maximum reflectance values when the optical stage is rotated under crossed polarizing filters [Murchison, 1978]. Anthracites, which are higher rank coals $\quad(>\sim 92 \mathrm{wt}-\%$ carbon), have larger aromatic clusters in their raw state ( $\sim 50$ aromatic carbon atoms and $\sim 600 \mathrm{amu}$ per cluster [Smith et al., 1994]) and show a degree of orientational order among clusters by high resolution TEM [Blanche et al., 1995], and bireflectance [Murchison, 1978].

Figure 7 show measurements of optical bireflectance as a function of carbonization temperature for three coals of various rank [Murchison, D., 1978]. These results are given as a function of temperature, rather than time, but are indicative of the solid structures observed during the various stages of carbonization, as they might occur during non-isothermal heat treatment. The lowest rank coal is isotropic it its initially state and remains essentially so during carbonization. The high-rank bituminous coal shows measurable anisotropy it its raw state, but loses this anisotropy at $400{ }^{\circ} \mathrm{C}$, and regains it at slightly higher temperatures. Apparently the increase in mobility on heating allows the structure to relax from a metastable configuration imposed by high lithostatic pressure to a near equilibrium isotropic configuration at 1 bar pressure. As heating continues, molecular weight growth proceeds until the planar structures reach the critical size (aspect ratio) for liquid crystal formation, and a highly ordered state develops. The bireflectance thus passes through a minimum, as seen in Fig. 6 in the curve labeled high-rank bituminous. The final degree of order is very high in the sample carbonized at temperatures above $400{ }^{\circ} \mathrm{C}$. The anthracite in raw form shows significant anisotropy, which is unaltered up to $600{ }^{\circ} \mathrm{C}$ and then slightly enhanced by higher temperature heat treatment. Despite the high degree of initial order, the anthracite does not develop the same degree of order as the high-rank bituminous coal. This is consistent with the observation

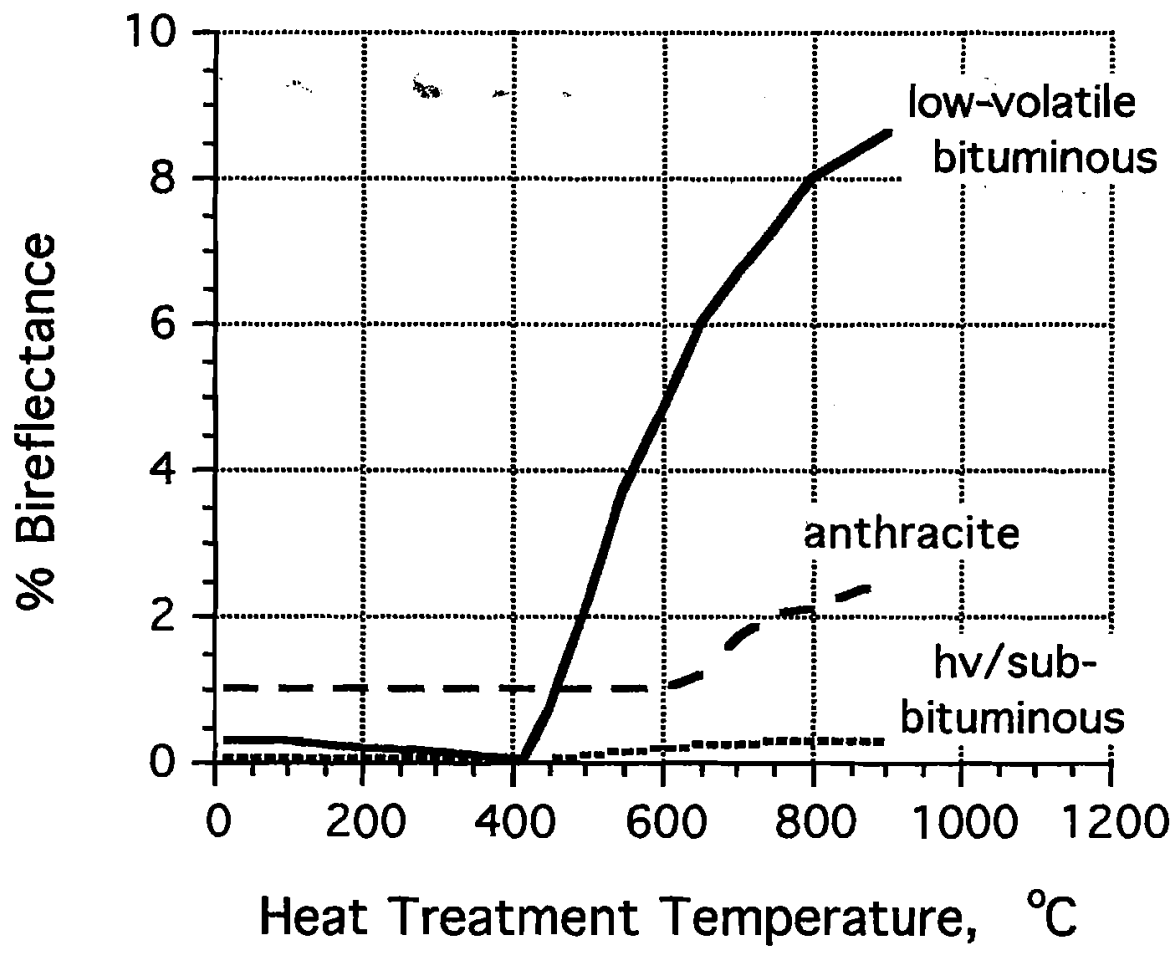

Figure 6 Measurements of optical bireflectance as a function of carbonization temperature for three important classes of coals from Murchison [1978]. 
that many anthracites are nongraphitizable [Blanche et al., 1995] (i.e. do not develop sufficient long range order on heating to produce graphite with good electrical and thermal properties). It is remarkable that many anthracites, despite the order and large cluster size in the raw state, form less ordered chars than do the high-rank bituminous coals. The general trends in carbon order across the coal rank spectrum are:

$$
\underset{\text { bituminous coals }}{\text { high-rank }}>\begin{gathered}
\text { many } \\
\text { anthracites }
\end{gathered}>\begin{gathered}
\text { low-rank } \\
\text { bituminous coals }
\end{gathered}>\begin{gathered}
\text { low } \\
\text { rank coals }
\end{gathered}
$$

The set of observations described above, including the qualitative features shown in Fig. $\quad 6$, are adopted as the targets for the extended model to be described in the next section.

\section{Simulations with initial anisotropy}

The basic numerical simulations were repeated with partially ordered initial states. Coordinates of the initial points were chosen randomly, as before, but initial growth directions were biased to have a preferred direction (non-zero $S_{o}$ ), giving rise to the curve labeled "initial anisotropy branch" in Fig. 4. At very low mobility, the initial orientational order in the coal is preserved intact in the char $(S=$ $S_{o}$ ). At higher mobility, the initial order is lost, leading to isotropic final chars (see the deep trough in the initial anisotropy branch of Fig. 4). At high mobilities the sample quickly loses memory of its initial order and the curve joins the previous curve for initially isotropic materials. The deep trough does not correspond to any known natural carbonaceous substance and therefore an additional factor must be added to the simple simulations to accurately simulate order development in high-rank fossil materials.

It was found that anthracitic order can be adequately represented by simulations in which the lines have a preferred initial direction (non-zero $S_{o}$ ) and non-zero initial length, $L_{o}$. Indeed, TEM [Blanche et al., 1995] and NMR [Smith et al., 1994] studies of raw anthracites indicate that significant aromatic cluster development has occurred during coalification. These simulations lead to the dashed curve in Fig. 8. The simulations show a gradual, monotonic increase in final order as the mobility increases, so that anthracites produce chars with long-range order intermediate between the high-rank bituminous coals and the low-rank materials. Equation 12 is consistent with the trends in Fig. 7 and the plot is labeled to summarize the connections between specific simulations and the various carbon types. 


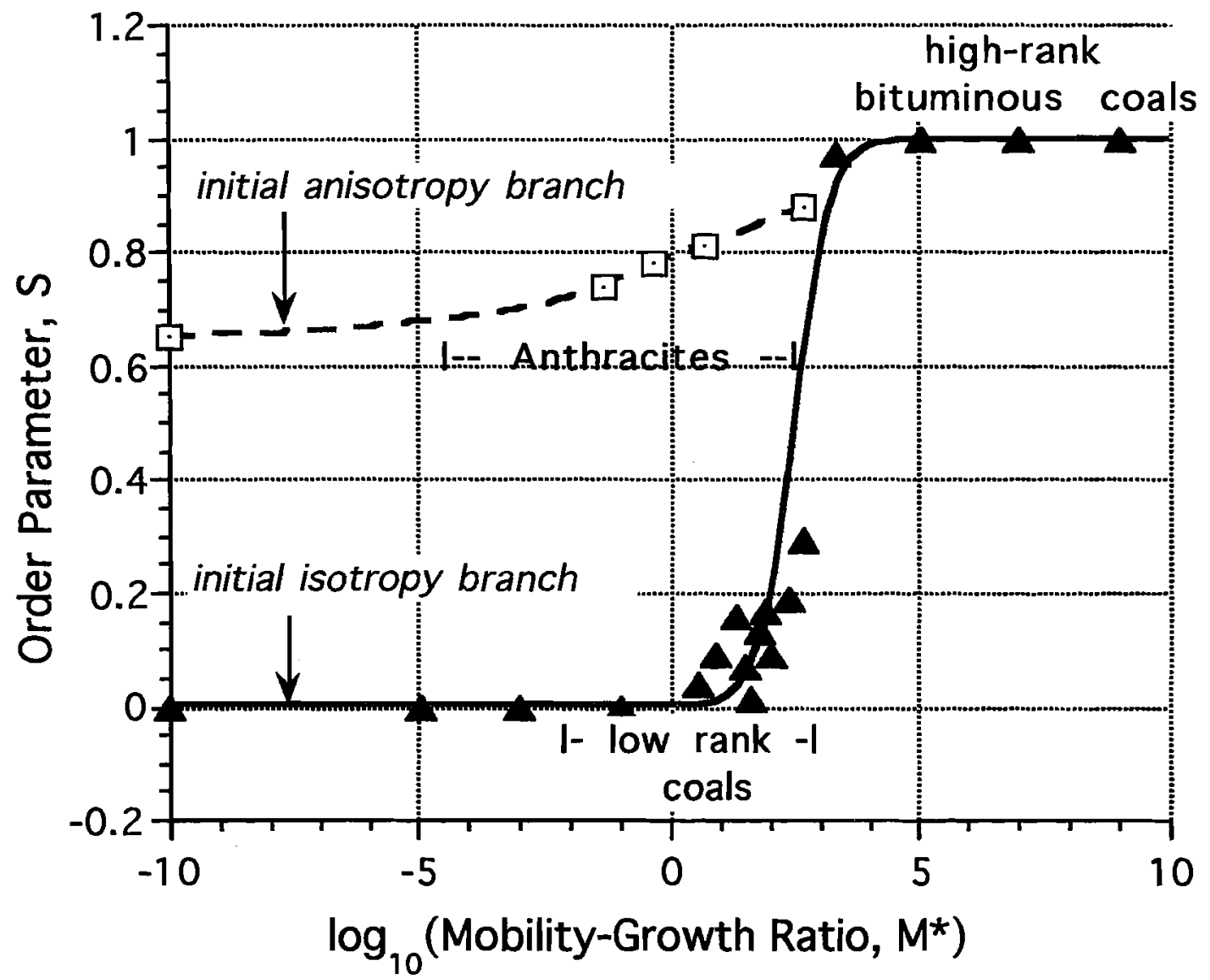

Figure 7 Summary of final states in numerical simulations starting from initially random states (isotropic parent materials) and initial ordered states (anisotropic parent materials). Long-range nematic order parameter in the final state is plotted as a function of the dimensionless mobility / growth ratio, $M^{*}$. The initially ordered states also have an initial length of $L_{o} / L_{b o x}=0.075$. Curve labels show the relation to carbonization processes. Type A carbons: isotropic solids formed through solid-state pyrolysis (e.g from lignites, woody tissue, oxygen-rich polymers); Type B carbons: anisotropic solids formed through liquid phase pyrolysis (pitches, polyaromatic compounds, coking coals); Type C carbons: anisotropic solids formed by solid-state pyrolysis of initially ordered precursors (e.g. anthracites).

Finally, Fig. 8 shows time dependent simulation results for three cases representing important classes of carbonizing systems. The simulations show that: (1) type A precursors (e.g. low rank coals) are nearly isotropic and remain so during carbonization, (2) type B precursors (e.g. high rank bituminous coals) lose their initial anisotropy, but regain it during the latter stages of carbonization, (3) type $C$ precursors (many anthracites) retain and slightly enhance their anisotropy during carbonization. Comparing Figs. 6 and 8, it is seen that the simple hard-line simulations also mimic these major trends seen in the bireflectance studies. 


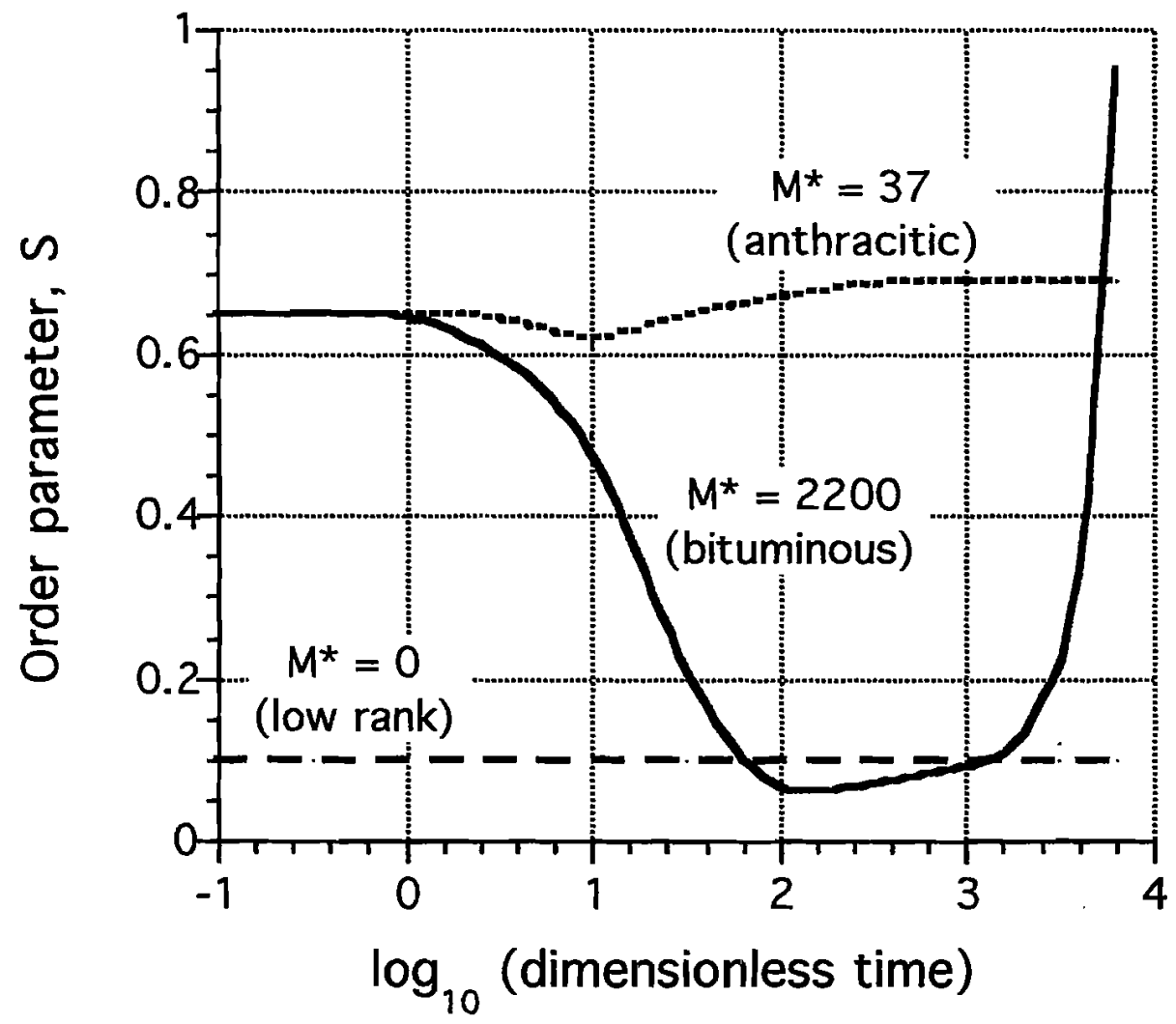

Figure 8 Time evolution of the long-range nematic order parameter in three simulations representing important classes of carbonization processes.

\section{Summary of Section 4.2}

A dynamic model of growth and random motion sf hard lines exhibits a kinetically influenced order/disorder transition followed by a gelation phenomenon to produce a variety of frozen configurations. The final length scale of orientational order is shown to depend sensitively on the ratio of rotational mobility and growth rate, low values of this ratio leading to the formation of small orientational order domains reminiscent of distinct crystallites in an isotropic solid. Potential applications of this toy model include non-equilibrium cooling of isotropic liquid phases, lyotropic transitions involving viscous solvents, and the synthesis of important classes of carbon materials. The simple simulations are sufficient to distinguish between optically isotropic carbons resulting from solid-state carbonization and optically anisotropic carbon resulting from fluid phase carbonization. After incorporation of initial orientational order and finite initial length, this remarkably simple model describes a number of key features of carbonization processes for organic precursors of varying type, including rank trends in ordered domain sizes and the measured time dependence of optical bireflectance in carbonizing coals. The present model cannot describe one important class of carbons, isotropic cokes, in which fluid phase pyrolysis yields carbons with short range crystallographic order. Modeling this important class of carbons will likely require explicit treatment of non-planar structures created by out-of-plane cross-linking functionalities such as ether, methylene, and thioether groups. 


\section{References for Section 4.2}

Bates, M.A., Luckhurst, G.R. J.Chem. Phys. 104 (17) 6696 (1996).

Barrall, E.M., Ch. 9 in Liquid Crystals: The Fourth State of Matter, (Saeva, ed.), Marcel Dekker, Inc. New York, 1979.

Blanche, C., Dumas, D., Rouzaud, J.N. Coal Science (Pajares and Tascon Eds.) Elsevier Science, Amsterdam, 1995, p. 43.

Chandrasekhar, S., Liquid Crystals, Second Edition, Cambridge University Press, Cambridge, 1992.

Clough, S.B., Blumstein, A., Hsu, E.C., Macromolecules 9123 (1976).

De Gennes P.G., Prost, J., The Physics of Liquid Crystals, Clarendon Press, Oxford, 1993.

Frenkel D., Mulder, B.M. Mol. Phys. 55 1171-92 (1985).

Gasparoux, H., Mol. Cryst. Liq. Cryst. 63 231-248 (1981).

Gay, J.G., Berne, B.J., J. Chem. Phys. 74(6) (1981).

Greinke, R.A., in Chemistry and Physics of Carbon, Vol. 24, Marcel Dekker, New York, 1994, p. 1.

Griffin, R.R., Scaroni, A.W., Walker, P.L.Jr. Carbon 29 (7) 991 (1991).

Hicklas, K., Bopp, P., Brickmann, J., J.Chem. Phys., 101 3157-3171 (1994).

Hurt, R.H., Hu, Y. "Thermodynamics of Carbonaceous Mesophase", Carbon, in press, 1998.

Lee, S.H., Kim, H.S., Pak, H., J. Chem. Phys. 97 (9) 6933 (1992).

Lewis, I.C., Carbon 16503 (1978).

Lewis I.C., Carbon 20 (6) 519 (1982).

Maier. W., Saupe, A.Z., Naturforsch. A13 564 (1958).

Marsh, Fuel, 52305 (1978).

Marsh, H., and Walker, P.L.Jr., Chap. 3 in Chemistry and Physics of Carbon, Vol. 15, (Walker and Thrower, eds.). Marcel Dekker, New York, 1979.

Murchison, D.G., Chap. 31 in Analytical Methods for Coal and Coal Products, Academic Press, New York, 1978.

Oberlin, A. in Chemistry and Physics of Carbon, Vol. 22, Marcel Dekker, New York, 1990.

Onsager, L., Ann. NY Acad. Sci. 51627 (1949).

Patrick, J.W., Reynolds, M.J., and Shaw, F.H., Fuel 52 198-204 (1973).

Rand B. in Handbook of Composites, Vol 1: Strong Fibers, (Watt and Perov, eds), North-Holland, Amsterdam, 1985, p. 495. 
Riggs, D.M., Diefendorf, R.J., Carbon '80 Baden Baden (Deutsche Keramische Gesellschaft), 1980, pp. 326-329.

Rouzaud, J.N., Oberlin, A., Chap. 17 in Advanced Methodologies in Coal Characterization, Elsevier, Amsterdam, 1990.

Shim, H.S., Hurt, R.H., Yang, N.Y.C. "A Methodology for Analysis of 002 LF Fringe Images and its Application to Combustion-Derived Carbons", Carbon, in press, 1999.

Shishido,M., Inomata, H., Arai, K., Saito, S., Carbon 35(6) 797 (1997).

Smith K.L., Smoot, L.P. Fletcher, T.H., Pugmire, R.J., The Structure and Reaction Processes of Coal, Plenum Press, NY, 1994.

Solomon, P.R., Best, P.E., Yu Z.Z., and Charpenay, S. Energy and Fuels, 6143 (1992).

Suuberg, E.M., M. Wojtowicz, and J.M. Calo, Carbon 27, 431 (1989).

\section{Nomenclature for Section $\mathbf{4 . 2}$}

$\vec{d} \quad$ director, mean orientational unit vector in liquid crystal phase

$f_{\text {ran }}(n) \quad$ order parameter for $\mathrm{n}$ randomly oriented objects (used as a

$G \quad$ correction factor for the small sample order parameter, $S(n)$, or $S(r))$

$G \quad$ growth rate

$L \quad$ line or layer length

$L_{b o x} \quad$ width of square simulation box

$L_{0} \quad$ initial line or layer length

$L_{o r} \quad$ characteristic length scale of orientational order

$\Delta L \quad$ growth step

$M^{*} \quad$ mobility / growth ratio

$N \quad$ number of lines in a simulation

$Q \quad$ order tensor used in the determination of $\vec{d}$

$R \quad$ rotation rate

$\Delta R \quad$ rotational step size

$S \quad$ order parameter

$S(l) \quad$ length-dependent order parameter

$S(n) \quad$ order parameter for sample of $n$ objects

$S_{o} \quad$ initial order parameter

$S_{\text {final }}$ order parameter in the final, frozen state

$t$ time

$\Delta t \quad$ time step

$\mathrm{T}^{*} \quad$ dimensionless time, $T^{*} \equiv t \cdot R$

$V \quad$ translational velocity

$V^{*} \quad$ translational / rotational velocity, $V^{*} \equiv V \rho^{I / 2} / R$

Greek symbols

$\theta_{i} \quad$ angle between the directional unit vectorfor object $i$ and the director

$\lambda$ eigenvalue in the equation determining $\vec{d}$

$\rho \quad$ number density of line centers (\# / length ${ }^{2}$ ) 


\section{Chapter 5: Combustion Modelling}

This Chapter presents a modeling study of the extinction behavior in the late stages of burnout based on work done at OSU, the University of Stuttgart (in collaboration with R. Essenhigh at OSU) and Brown. This work has led to the development of the Carbon Burnout Kinetic Model Version 8 (CBK8), with improved ability to describe the late stages of combustion. The development and validation of CBK8 is also described in the chapter.

\section{Technical Background: Mechanisms of Extinction and Near-Extinction}

Previous studies have documented large decreases in particle temperature in the late stages of pulverized coal char combustion. The first relevant observation was made by Mitchell [1990], who used in situ particle sizing micropyrometry to observe the sudden appearance of particles near the local gas temperature after long residence times in an optically accessible entrained flow reactor. The author attributed this observation to ash-rich particles in the final stages of burning. In a subsequent study [Hurt and Davis, 1994] the optical data were analyzed further to show that many of these particles have emissivities indicative of a carbon-rich composition. Also, in separate experiments with captive particles, extinction-like events were directly observed with a long-focal-length video microscopy system recording both reflected visible light and nearinfrared incandescent images [Hurt and Davis, 1994]. It was clear that combustion occurs in two distinct stages: a rapid high temperature stage consuming $60-80 \%$ of the char carbon, followed by an extinction-like event and a final burnout phase occurring at or below the local gas temperature. The final burnout phase greatly increases the time required to reach the very high conversions required in industrial practice $(>99 \%)$. In the original paper [Hurt and Davis, 1994], the mechanism responsible for the extinction-like events was not identified.

Recently, a carbon burnout model was proposed that incorporates submodels of annealing, ash effects, and statistical kinetics [Hurt et al., 1998]. This model, CBK6, predicts extinction-like events under certain conditions, but only for ash-containing particles. In further work, Lunden et al. [1998] did not find well developed ash films in the combustion of Pittsburgh seam coal at sufficient low conversion to explain the extinction-like events. Also, further captive particle imaging experiments directly reveal extinction events even for particles with little or no ash [Hurt and Davis, 1999]. It appears likely that CBK6 lacks at least one extinction mechanism and compensates for this by overestimating the effect of ash. The same set of experiments showed extinction behavior at gas temperatures from 1150 - $1250 \mathrm{~K}$ to be precursor dependent, occurring for bituminous coal chars but not for lignite chars [Hurt and Davis, 1999], a feature that cannot be predicted by CBK6. One of the latter papers cites the need for more work on the mechanisms of extinction [Hurt and Davis, 1999].

Very recently, Essenhigh, Klimesh and Förtsch [1999a,b] have carried out analyses of internal reaction and diffusion in char particles and concluded that the Zone I reaction mode (corresponding to complete oxygen penetration) can occur under pulverized coal combustion conditions. This is contrary to the common approach for describing pulverized coal char 
combustion which implicitly assumes the reaction to be in Zone II, where the oxygen penetration depth is much less than the particle radius and the kinetics can be expressed using global rate coefficients based on external area that lump internal reaction with pore diffusion effects. These authors also conclude that accounting for the Zone II to Zone I transition is necessary to properly model extinction behavior in the late stages of combustion [Essenhigh et al., 1999b].

The present report examines the complete mass and energy transport processes that govern char particle temperature, and considers a number of separate deactivation mechanisms that can lead to extinction and extinction-like phenomena. The goal is to develop a theory that explains all the observations related to extinction-like behavior in the experimental studies cited above. The report focuses primarily on two sets of combustion conditions: (1) pulverized coal char combustion (PC) conditions defined by $20 \mu \mathrm{m}<\mathrm{d}_{\mathrm{p}}<150 \mu \mathrm{m}, 1500<\mathrm{T}_{\mathrm{g}}<2000 \mathrm{~K}, 3 \%<\left[\mathrm{O}_{2}\right]$ $<12 \%, 500 \mathrm{~K}<\mathrm{T}_{\mathrm{w}}<\mathrm{T}_{\mathrm{p}}$, and (2) captive particle imaging (CPI) conditions as used in the Sandia experiments [Hurt and Davis, 1994, 1999] defined by $100 \mu \mathrm{m}<\mathrm{d}_{\mathrm{p}}<300 \mu \mathrm{m}, 900<\mathrm{T}_{\mathrm{g}}<$ $1250,3 \%<\mathrm{P}<19 \%, \mathrm{~T}_{\mathrm{w}} \sim 500 \mathrm{~K}$. A model will be proposed that successfully describes:

- extinction of chars from all coal ranks under PC conditions, as well as quantitative mass loss profiles at high conversion,

- extinction of bituminous but not of lignitic chars under CPI conditions

- extinction of low-ash content particles under CPI conditions

- ignition delays and very early extinction for high rank coal chars under CPI conditions,

- absolute char reactivities and their rank dependencies both under PC conditions (as measured at Sandia [Hurt and Mitchell, 1992], and under TGA conditions $\left(500^{\circ} \mathrm{C}\right)$ as measured by Jenkins et al. [1973].

\section{Theory}

Equations $1-4$ represent the single particle energy balance in simple approximate form, assuming nonabsorbing, nonemitting gases, no interparticle interactions (dilute solids limit), and omitting the small correction for Stefan flow.

$$
m_{p} C_{p} d T_{p} / d t=Q_{r a d}+Q_{c o n v}+Q_{r x n}
$$

where

$$
\begin{gathered}
Q_{\mathrm{rad}}=A \sigma \varepsilon\left(\mathrm{T}_{\mathrm{p}}^{4}-\mathrm{T}_{\mathrm{w}}{ }^{4}\right) \\
\mathrm{Q}_{c o n v}=A U\left(\mathrm{~T}_{\mathrm{p}}-\mathrm{T}_{\mathrm{g}}\right) \\
\mathrm{Q}_{\mathrm{rxn}}=\mathrm{R}\left(-\Delta \mathrm{H}_{\mathrm{rxn}}\right) / \mathrm{MW}_{\mathrm{c}}
\end{gathered}
$$

and where the overall heat transfer coefficient, $U$, in the presence of an ash film, is given by:

$$
U=\frac{\left(\frac{d_{p}}{d_{c}}\right)^{2}}{\left(\frac{1}{h_{\text {ext }}}+\frac{1}{h_{\text {int }}}\right)}
$$




$$
\begin{gathered}
h_{\text {ext }}=\frac{\lambda_{g} N u}{d_{p}} \\
h_{\text {int }}=\frac{2 \lambda_{a}}{\delta} \frac{d_{c}}{d_{p}} \\
\lambda_{a}=(1-\theta) \lambda_{a, \text { true }}+\theta \lambda_{g}
\end{gathered}
$$

Here $\lambda_{\mathrm{a}}$ is the thermal conductivity of the porous ash, given as the volumetric average of the gas phase conductivity, $\lambda_{\mathrm{g}}$, and the conductivity of the fully dense mineral phase, $\lambda_{\mathrm{a}, \text { true- }}$

Equations 5-8 incorporate the influence of an ash film on heat transfer. The effect of ash is widely recognized to be insignificant in the early to middle stages of combustion, but can become important at high conversion where ash is the majority component. For example, high depth of field optical micrographs of highly reacted char particles $(X \sim 96 \%)$ after three passes through the Sandia entrained flow reactor show that the ash is partially fused, is often in intimate contact with the remaining carbon, and occurs in irregular geometries. At this very high conversion, much of the carbon appears to be imbedded in or beneath glassy material, which is expected to significantly alter heat and mass transfer to and from the reacting surfaces. It is not practical to describe the full complexity of these composite structures, so a representative onedimensional spherical geometry is chosen for analysis.

Analysis of equation 5 reveals that ash can either increase or decrease heat transfer rate depending on the value of the Nusselt number for convective loss. In the small particle limit (Nu $=2$ ) the ash film always enhances heat transfer, acting in the manner of a fin conducting heat from the carbon surface to a larger external area. It is surprising that a porous glassy layer does not act as a net insulator, but this can be physically rationalized by considering that the ash film replaces a gas film, which in the small particle limit $(\mathrm{Nu}=2)$ is effectively stagnant and is an even better insulator by virtue of its lower thermal conductivity.

\section{Internal Reaction and Diffusion}

This section presents a simple kinetic model for the overall burning rate, $\mathbf{R}$, appearing in Equation 1. For this work, the single particle combustion rate, $\mathrm{R}$ (gm carbon / sec), is modeled as:

$$
R=\eta k_{o} S e^{-E / R T} T_{p} P_{S}^{n} m_{p}
$$

where $k_{o}$ is the preexponential factor for the surface rate constant $\left(\mathrm{gm} \mathrm{carbon} / \mathrm{s}-\mathrm{cm}^{2}-\mathrm{atm}^{\mathrm{n}}\right), \mathrm{S}$ the total surface area / mass, $\left(\mathrm{cm}^{2} / \mathrm{gm}\right), \mathrm{E}$ the intrinsic activation energy, $\mathrm{P}_{\mathrm{S}}$ the oxygen partial pressure at the outer particle surface (atm), $n$ the empirical apparent reaction order for the range of $P_{s}$ examined, $m_{p}$ the carbon mass in the particle (gm), and $\eta$ is the dimensionless effectiveness factor:

$$
\eta=\frac{1}{\phi}\left[\operatorname{coth}(3 \phi)-\frac{1}{3 \phi}\right]
$$


where $\phi$ is a generalized Thiele modulus for spheres [Froment and Bischoff, 1990]:

$$
\phi=\frac{d_{p}}{6}\left[\frac{(n+1) k_{0} S \rho}{2 D_{\text {eff }}}{\underline{\left(P_{s} / R T\right)^{(n-1)}}}^{1 / 2}\right.
$$

Equations 10 and 11 yield good approximate values of $\eta$ for all exponents $\mathrm{n}$ in power law kinetics [Froment and Bischoff, 1990]. The oxygen partial pressure at the particle surface, $\mathrm{P}_{\mathrm{S}}$, is found by solution of mass transfer relations for boundary layer diffusion and ash film diffusion in series, as described elsewhere [Hurt et al., 1998]. This solution requires knowledge of the ash film thickness, $\delta$, and porosity, $\theta_{\mathrm{af}}$.

It has been noted previously that the parameters $\mathrm{k}_{\mathbf{0}}$ and $\mathrm{S}$ appear together in this formalism and for some purposes can be advantageously treated as a single parameter, $\mathrm{k}_{\mathrm{o}} \mathrm{S}$ - a "mass specific intrinsic reactivity". The lumped parameter $\mathrm{k}_{0} \mathrm{~S}$ is a property of a carbon sample that can be directly measured (e.g. in TGA experiments). The subsequent decomposition into the individual parameters $k_{o}$ and $S$ requires choice of an appropriate surface area (subject to uncertainty for microporous chars) and, while often providing detailed insight into reaction behavior, offers no particular advantage for the specific analyses in this paper.

The ash-forming mineral matter in coals and biomass can affect combustion through catalysis, as well as through modification of transport processes. In the present analysis, catalytic effects, which are primarily associated with atomically dispersed or very fine grained inorganic matter, are incorporated into the mass specific intrinsic reactivity, $\mathrm{k}_{\mathrm{o}} \mathrm{S}$, characteristic of a given fuel, while explicit models are developed for the transport effects associated with the bulk, granular mineral matter. Ash effects on mass transfer have been treated previously [Hurt et al., 1998], while the heat transfer effects are represented by Eqs $5-8$.

The major challenge in applying this formalism is the estimation of the effective diffusivity, $D_{\text {eff, }}$ a property of the complex inhomogeneous pore structure of chars. Pores diameters span up to 5 orders of magnitude, from $4 \mathrm{~A}$ micropores to $40 \mu \mathrm{m}$ vesicles. Diffusion limitations may occur in pores that feed the particle interior or in microporous regions lying between the large feeder pores [Satterfield, 1981, Carberry, 1962]. Of primary interest here are the transport limitations to the particle interior, as these occur over the whole particle length scale and must be described explicitly to predict the influence of particle diameter on overall rate. (Diffusion limitations in microporous grains on the other hand are not materially affected by particle diameter, and their effects can often be absorbed into the intrinsic surface rate coefficient [Gavalas, 1980]. We therefore concentrate on describing radial concentration gradients that reflect transport limitations to the particle interior.

Transport to the particle interior is believed to occur primarily through large feeder pores in which diffusion occurs in or near the molecular regime [Simons, 1983]. Note that a significant fraction of the porosity and almost all the surface area in chars lies on the surfaces of fine (microand meso-) pores, but these pores are not as effective providing long-path-length transport to the particle interior [Simons, 1983] due to slow diffusion by the Knudsen or restricted mechanisms. Simple calculations with the classical parallel path pore model [Satterfield, 1981] confirm that 
the meso- and micro-pores contribute little to overall transport for pore size distributions typical of flame chars.

With this simplifying assumption, the effective diffusivity for transport to the particle interior will be modelled as $D_{\text {eff }}=D_{M} \theta f / \tau$, where $D_{M}$ is the molecular diffusivity, $f$ is the fraction of the total porosity in feeder pores and $\tau$ is the tortuosity. The advantage of this simple approximate formulation is that $D_{M}$ and $\theta$ are known at all points from $T, P, d_{p}$, and particle density, and the complex nature of the pore structure in char is embodied in the ratio $f / \tau$ or ( $\tau / f$ ) which can be treated as a single empirical parameter. In practice the parameter ( $\tau / f$ ) will absorb other approximations made here, such as the influence of partial Knudsen diffusion in the smaller feeder pores. It will be seen later that a single, physically realistic value of the intraparticle transport parameter, ( $\tau$ / f ), without adjustments for rank or temperature, allows the description of reactivity and extinction behavior over a wide range of fuels and conditions.

\section{Pseudo Steady State Solutions: Extinction and Near-Extinction}

The features of the complex equation set (Eqs. 1 - 11) are best understood by examining certain simplified cases. In particular, if the term $\mathrm{m}_{\mathrm{p}} \mathrm{C}_{\mathrm{p}} \mathrm{dT} / \mathrm{dt}$ (representing the particle thermal mass) in Eq. 1 is small, the differential equation is transformed into an algebraic equation which can be solved jointly with the remaining 10 equations to yield the instantaneous particle temperature, $T_{p}$. Indeed in many cases the term $m_{p} C_{p} d T / d t$ is relatively small during the main portion of the combustion process, causing the particles to burn at a pseudo steady state temperature, sometimes referred to as the temperature of thermal equilibrium. This section analyzes the equation set in pseudo steady state and presents results which help elucidate the nature of extinction processes.

The basic relations (Eqs. 1 - 11) can be represented in the form of two separate curves of heat flow, $Q$, vs. particle temperature for each of two sets of conditions in pseudo steady state. The first curve gives the heat release rate, $Q_{r x n}$, by solving the reaction and mass transfer relations alone for known $T_{p}$. The second curve represents the energy balance and yields Qloss. The intersection of the two curves gives the particle temperature in the limiting case of small $m_{p} C_{p}$ $\mathrm{dT}_{\mathrm{p}} / \mathrm{dt}$.

Such a plot shows only one steady state under PC conditions, while at lower gas temperatures (CPI conditions) three steady states are possible. In the latter case the upper and lower steady states are stable with respect to small perturbations, while the middle steady state is unstable. As a result, the upper and lower are both observable in practice and the appearance of one or the other depends on previous particle history, or initial conditions. Careful exploration of the entire range of PC conditions (as stated in background) did not reveal any examples of multiple steady states. Indeed, even in the CPI range, unusually high oxygen concentrations are required to bring about the phenomenon of multiple steady states. We can state the general conclusion that both low gas temperatures $(<1300 \mathrm{~K})$ and high oxygen concentrations $(>12 \%)$ are required for multiple solutions to the single particle energy balance.

An analysis was performed to illustrate the effect of decreasing gas temperature, $T_{g}$, on particle temperature, $T_{p}$, under CPI conditions. A gradual decrease in gas temperature is found to cause 
a discontinuous change in the pseudo steady $T_{p}$ due to loss of the upper point of intersection. Under PC conditions multiple steady states are not observed and the particle temperature drops gradually and continuously, eventually reaching the inert particle limit when the reaction is too slow to significantly influence the energy balance. To distinguish these two phenomena, we will refer only to the first discontinuous change as an "extinction". The mathematically continuous change, if it occurs over a narrow time interval can lead to a rather abrupt transition and will be referred to as a "near extinction". If the temperature drop occurs over a significant time interval, it deserves no special designation.

In practice, the temperature drop during a true extinction event will be abrupt, but not instantaneous due to the thermal lag associated with the transient term, $m_{p} C_{p} d T_{p} / d t$. As a result, it is not always possible to distinguish a true extinction event from a near-extinction event based on data alone - mathematical analyses may be required in some cases to properly classify an abrupt temperature drop using these definitions.

Figure 1 shows the effect of ash film thickness on the pseudo steady state particle temperature. The thick dashed curve gives the combined effect of heat and mass transfer limitations. The

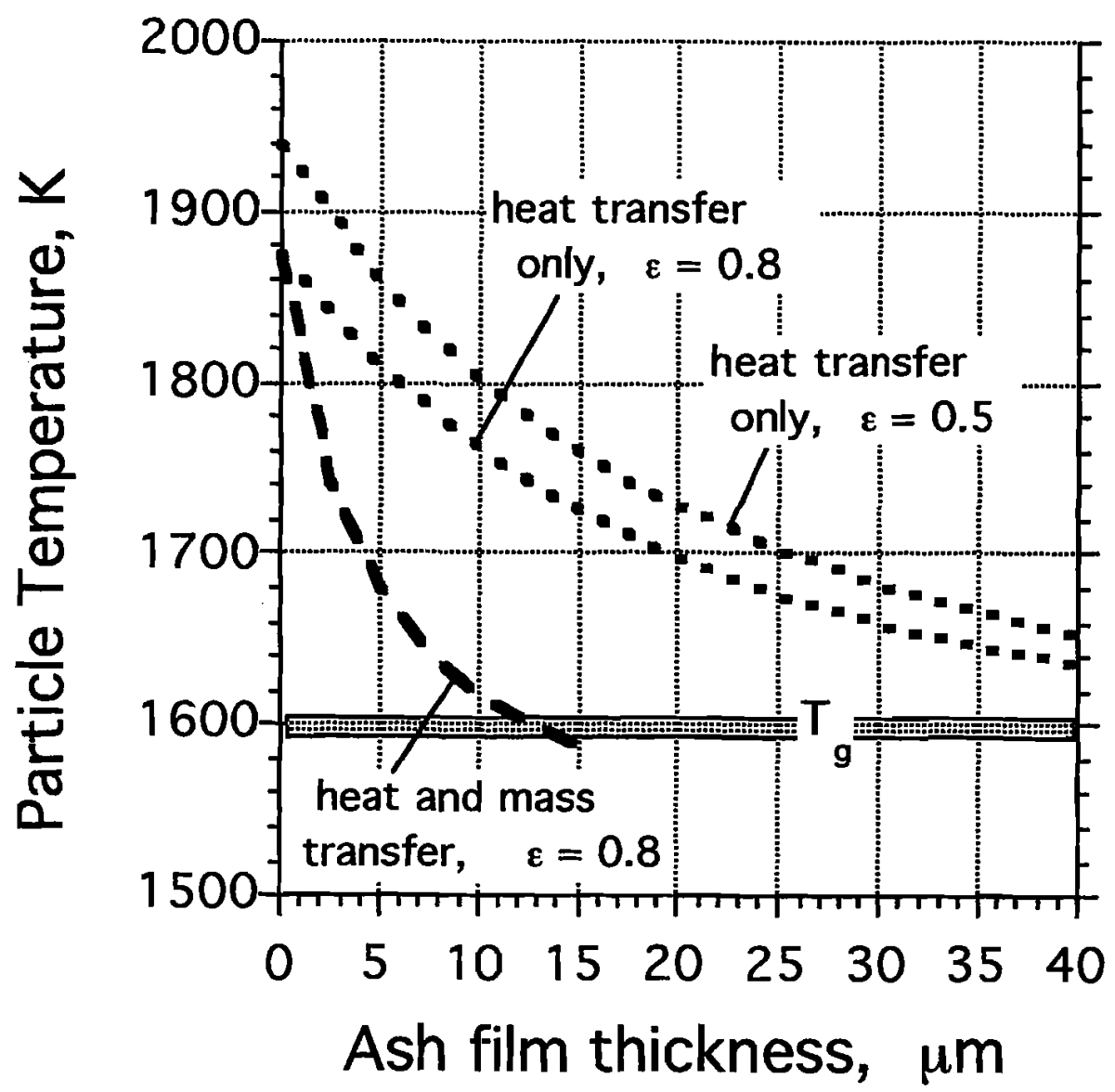

Figure 1 Effect of ash film thickness on pseudo steady state particle temperature under an example set of conditions. Carbon core diameter: $50 \mu \mathrm{m}$; gas temperature: 1600 $\mathrm{K}$; wall temperature: $500 \mathrm{~K}$; oxygen concentration: 12 mole- $\%$; ash film porosity: $50 \%$, thermal conductivity of fully dense ash film: $0.005 \mathrm{cal} / \mathrm{cm}-\mathrm{s}-\mathrm{C}$. 
dotted curves give the heat transfer effect only for a total emissivity characteristic of carbon (0.8) and of ash (0.5). The plot shows that fully developed ash films thicker than $5 \mu \mathrm{m}$ can have a large effect on particle temperature (and burning rate). The magnitude of the mass transfer effect depends critically on the geometry of the composite particle - the solution shown is for a fully encapsulated carbon core in spherical geometry. In this geometry the mass transfer limitation is much more pronounced than the heat transfer enhancement. If the ash does not effectively encapsulate the carbon, the heat transfer effect may be similar, but the mass transfer limitation will be much less pronounced. Analyses were performed to study how the pseudo steady state particle temperature changes under conditions of decreasing particle size, gas temperature, and reactivity. Significant decreases in particle temperature are observed in all cases. Under CPI conditions the changes are discontinuous extinction events while at the higher temperature PC conditions the changes are continuous near-extinction events. The classification of the CPI curves as true extinction events has been confirmed by separate analysis of the $Q_{\mathrm{rxn}}$ and $\mathrm{Q}_{\text {loss }}$ curves. These results will be useful in the interpretation of the complete numerical simulations in the next section.

\section{Numerical Simulations}

A large set of comprehensive single-particle simulations were carried out, accounting for internal reaction and diffusion, annealing, and ash effects. The goal of these simulations is to determine if a single set of coal-general parameters can describe the complete set of observations summarized in the introduction. The main parameters varied were $\tau / \mathrm{f}$, which establishes the effective diffusivity; and $\delta_{\mathrm{m}}$ and $\theta_{\text {taf }}$ which give the ash grain size, and fully developed film porosity respectively. Table 1 summarizes the best value of these three parameters and gives other important constants that fully define the new model, CBK Version 8.

Table 1

\section{Recommended CBK8 Model Parameters}

\section{Adjusted parameters}

Name

carbon pore

structure parameter

mineral grain size

porosity of

thick ash film

correlation for

initial reactivity:

$\begin{array}{ll}\frac{\text { Symbol }}{\tau / \mathrm{f}} & \frac{\text { Value }}{6} \\ \delta_{\mathrm{m}} & 5 \mu \mathrm{m} \\ \theta_{\mathrm{taf}} & 0.17 \text { (PC temperatures) } \\ & >0.5 \text { (CPI temperatures) }\end{array}$

$\log _{10}\left(k_{o} S_{o}\right)=14.97-0.0764(w t-\% C)$

(daf in parent coal)

Additional parameters

intrinsic reaction order $\mathrm{n} \quad 0.5$

intrinsic activation energy E $\quad 35 \mathrm{kcal} / \mathrm{mol}$

empirical constants ${ }^{\dagger} \quad A_{c} \quad 200$

for $\mathrm{CO} / \mathrm{CO}_{2}$ ratio $\quad \mathrm{E}_{\mathrm{c}} \quad 9 \mathrm{kcal} / \mathrm{mol}$

$\dagger$ derived from summary of literature kinetic data by Skokova [1997]. 
Figures 2 - 5 show example predictions generated with CBK8 and the above parameter set. Figure 2 compares the measured and predicted mass loss profiles for Illinois \#6 and Beulah lignite. The model correctly predicts the shape and magnitude of the mass loss curves at all times and conversions (to 99\%). Also shown on Fig. 2 are the predictions for hypothetical chars with no mineral content. The two predictions for Illinois \#6 are the same, indicating that mineral effects are not responsible for the near-extinction of this char. The two predictions for Beulah lignite do show an effect of mineral matter on near-extinction. The different behavior of the two coals can be understood as follows. The much more reactive Beulah lignite maintains high burning rates in zone II reaction mode until high conversion (90-95\%), at which point the abundant mineral matter modifies the heat and mass transfer to promote near-extinction. A similar effect is predicted for larger particles $(\sim 300 \mu \mathrm{m})$ which develop an ash film before they are small enough to approach the Zone I regime. In contrast, the less reactive llinois \#6 chars extinguish earlier by an mechanism that doesn't involve ash, and because the subsequent reaction occurs essentially in zone I, transport processes are no longer limiting and the increasing ash content has no effect on the rate, even as burnout advances well into the ash-rich region.

Insight into the underlying cause of the near-extinction event for Illinois \#6 coal chars is provided by Fig. 3. Particle temperature is seen to drop continuously after $30 \%$ conversion, and the drop becomes steep near $70-80 \%$ conversion, the point where the knee in the curve is observed in Fig. 2. This decreasing temperature is accompanied by a decrease in $R / R_{\max }$ to near zero, indicating the disappearance of boundary layer concentration gradients, and an increase in effectiveness factor, $\eta$, to near unity, indicating the disappearance of internal gradients. These particles begin their combustion lifetimes reacting in zone II/III transition region, and fall to zone I conditions during the near-extinction event. The underlying cause of the near-extinction in this case is a decreasing particle diameter (see Figure) with a lesser contribution from annealing-driven decreases in reactivity, $\mathrm{k}_{0} \mathrm{~S}$, and from decreasing density. It is found in most simulations that annealing occurs early, causing $k_{0} S$ to change rapidly at low conversion, but slowly thereafter. In these simulations, annealing is rarely if ever the primary cause of near-extinction events, which occur later in combustion (compare the shapes of the $T_{p}$ and $\mathrm{k}_{\mathrm{o}} \mathrm{S}$ curves in Fig. 3 ).

The role of the Zone II / I transition can be understood as follows. As diameter decreases during combustion, burning rates, heat release rates, and heat transfer rates decrease in absolute units (e.g. Watts). Zone II reaction rates scale as $\mathrm{d}_{\mathrm{p}}{ }^{2}$ (proportional to external area), while Zone I rates scale with the particle mass, or $\mathrm{d}_{\mathrm{p}} 3$. As the reaction approaches and enters Zone $I$, the reaction rates become more sensitive to diameter and begin to decrease more rapidly with decreasing particle mass. Accounting for the zone II / I transition is found to be important for the proper prediction of many near-extinction events, in agreement with the conclusions of Essenhigh and coworkers [1999a,b]. Additional simulations with constant particle diameter indicate that extinction can also occur by decreases in particle density, accompanied by a similar transition from Zone II to Zone I. 

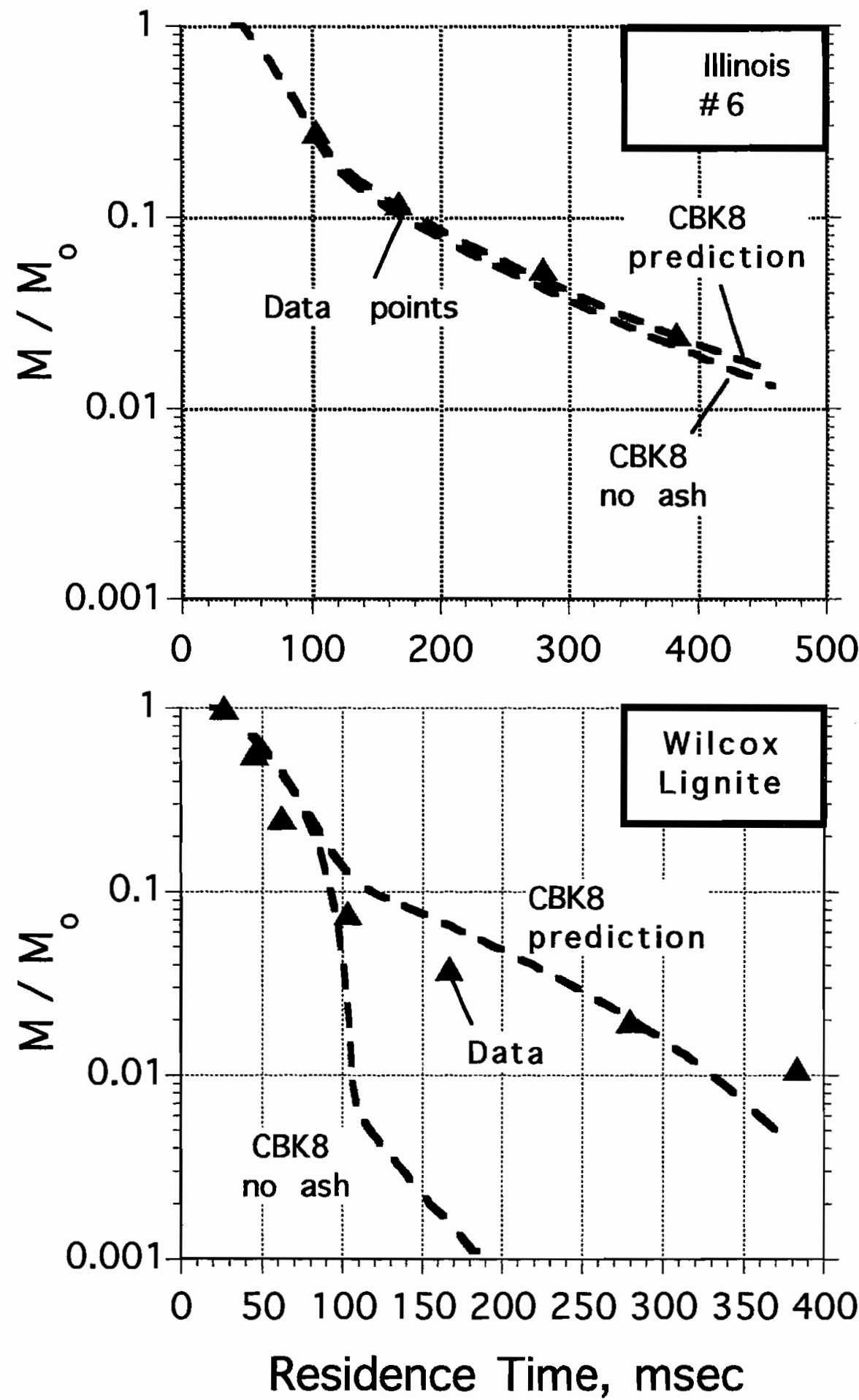

Figure 2. Overall carbon conversion as a function of time determined by extractive sampling in a hybrid flame-driven entrained flow reactor with supplementary electrical heating. Oxygen concentration is $12 \%$ and gas temperature is $1450 \mathrm{~K}$. Data taken from reference [Hurt et al., 1998]; data fits from the present paper (CBK8). 


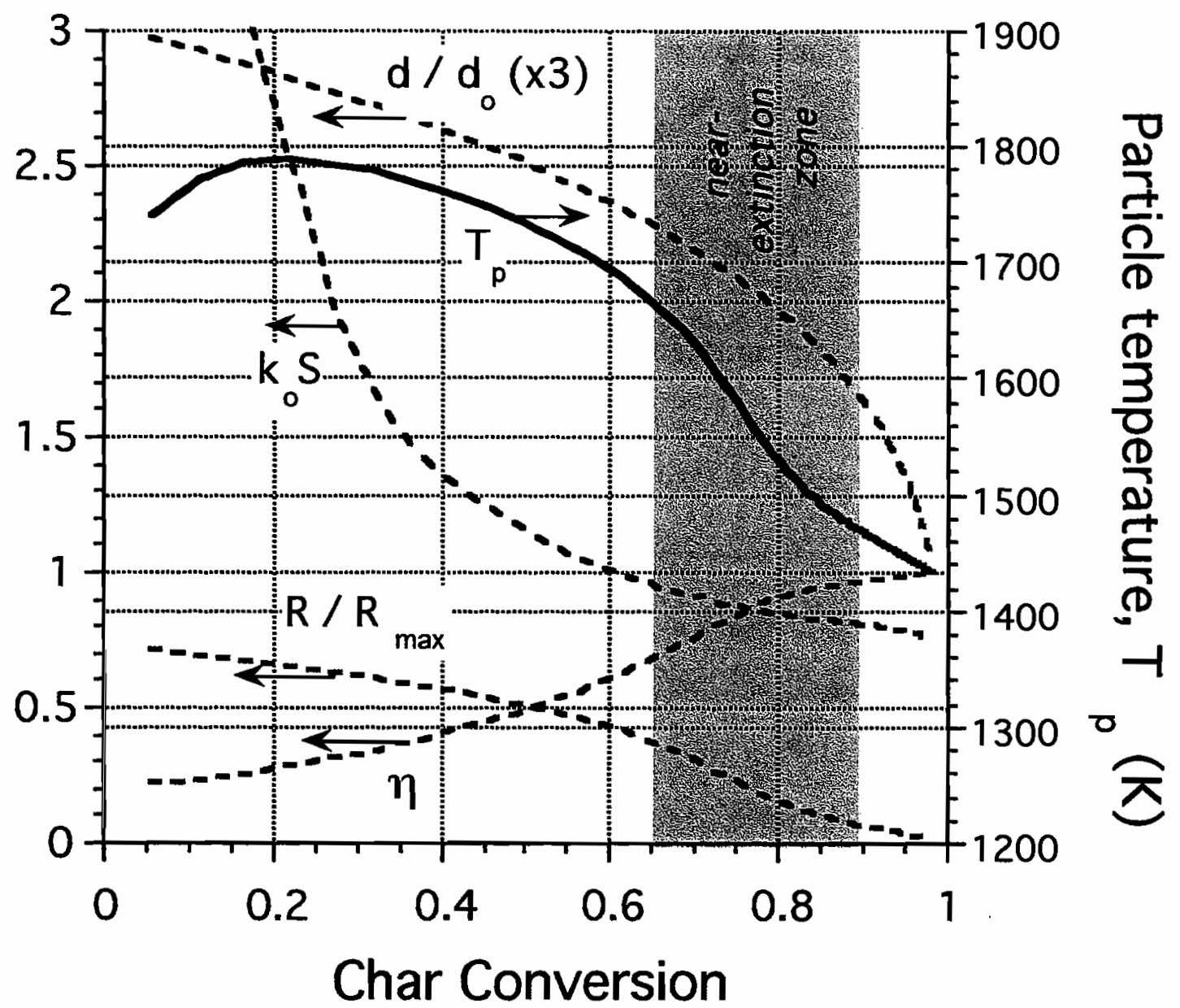

Figure 3. CBK8 predictions of Illinois char behavior giving insight into reaction zones and mechanisms of near-extinction. Conditions identical to those in Fig. 8. $R_{\max }$ is the theoretical diffusion limited rate, and $R / R_{\max }$ is a measure of the extent of approach to this limit (a measure of the severity of boundary layer diffusion limitations ranging from 0 to 1 ).

The same parameter set successfully predicts rank-dependent extinction under CPI conditions (see Fig. 4). The lignite char maintains a high particle temperature throughout burnout due its intrinsically high reactivity which maintains zone II/III conditions even for smaller particles. The ash effect which is needed to predict the mass loss curve for the lignite in the entrained flow reactor (Fig. 2) does not have a large effect on the CPI predictions in Fig. 4 due to the lacy, unfused, and highly porous nature of the ash at these low temperatures.

Finally, it has been noted that the Pocahontas char shows an unstable behavior under CPI conditions with 19 mol-\% oxygen, in the form of delayed ignition [Hurt and Davis, 1999] or 
even multiple ignition and extinction events. Calculation of the $\mathrm{Q}_{\text {loss }}$ and $\mathrm{Q}_{\mathrm{rxn}}$ curves for the Pocohontas CPI conditions reveal the two curves to be nearly tangent over a wide range of particle temperature. This tangency produces wide swings in particle temperature upon slight perturbation of conditions, providing a convincing explanation for the observed erratic behavior. Finally, Fig. 5 shows CBK8 predictions of absolute burning rates for a range of coals of various rank under two sets of conditions: PC conditions in the Sandia entrained flow reactor [Hurt and Mitchell, 1992] and very low temperature conditions $\left(500^{\circ} \mathrm{C}\right)$ in TGA data of Jenkins et al. [1973]. The successful fit of the Sandia data at high temperature is not significant in itself, as this very data was used to develop the reactivity correlation in CBK8 (see Table 1). In contrast, the successful fit to the TGA data is very encouraging, as the same parameter set was used (see Table 1), which is tantamount to an a priori extrapolation of over $1000{ }^{\circ} \mathrm{C}$ in particle temperature and 4 to 5 orders of magnitude in reaction rate. Note that both the annealing model and the explicit description of internal reaction / diffusion were important for achieving this

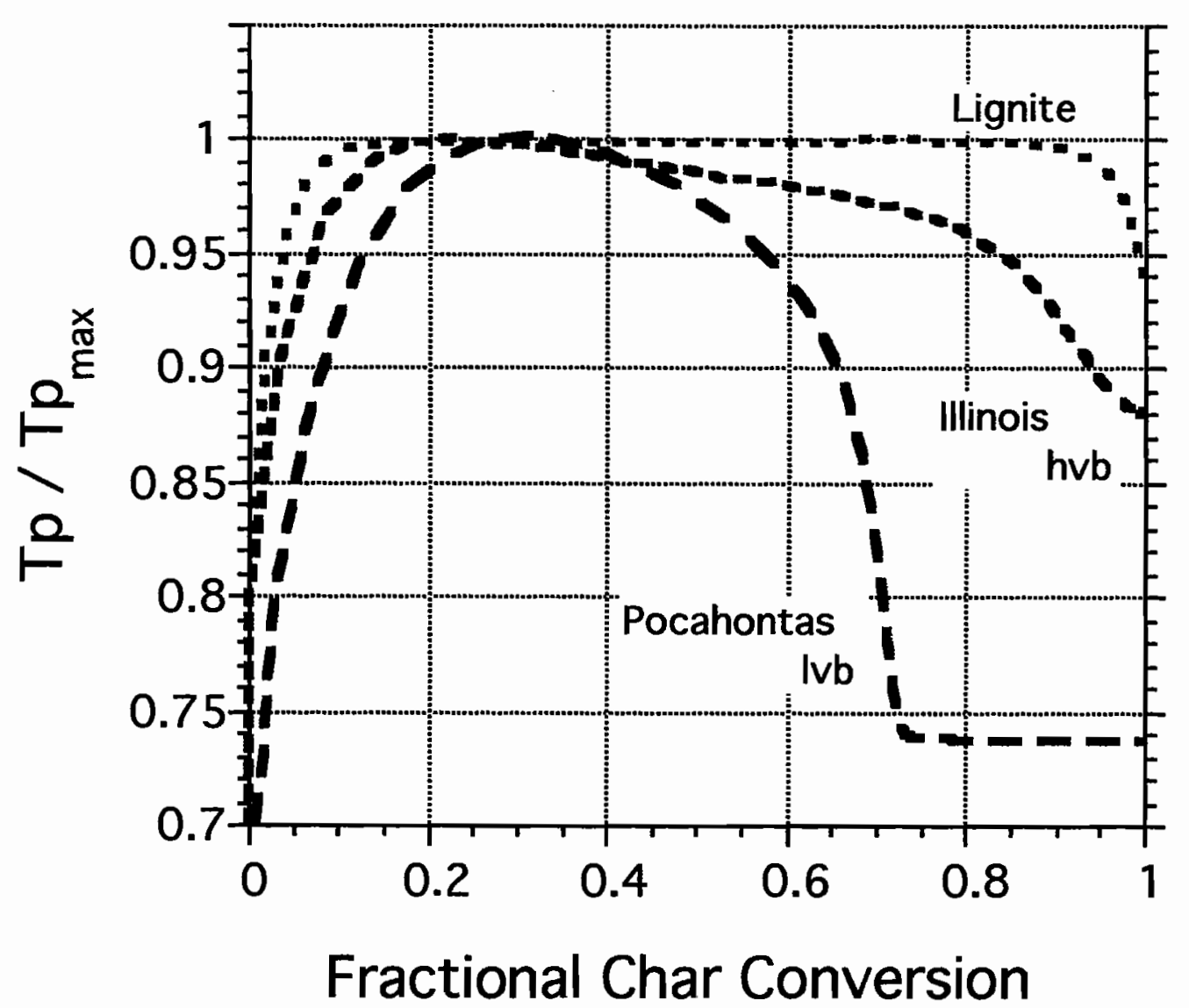

Figure 4. CBK8 predictions of particle temperature histories under CPI conditions, showing rank-dependent extinction behavior. Conditions: lignite: 3 mole-\% oxygen, $1175 \mathrm{~K}$ gas temperature; Illinois \#6: 6 mole- $\%$ oxygen, $1250 \mathrm{~K}$ gas temperature; Pocahontas: $19 \%$ oxygen, $1250 \mathrm{~K}$ gas temperature. 
unification over a wide temperature range ${ }^{\dagger}$. The results in Figs. $2-5$ were generated using the common parameter set presented in Table 1. Critical examination of these parameters shows them to be physically realistic. The ash grain size is 5 microns and the ash porosities are reasonable for the partially fused material seen in Fig. 2 at high temperature $(\theta=0.17)$ and the

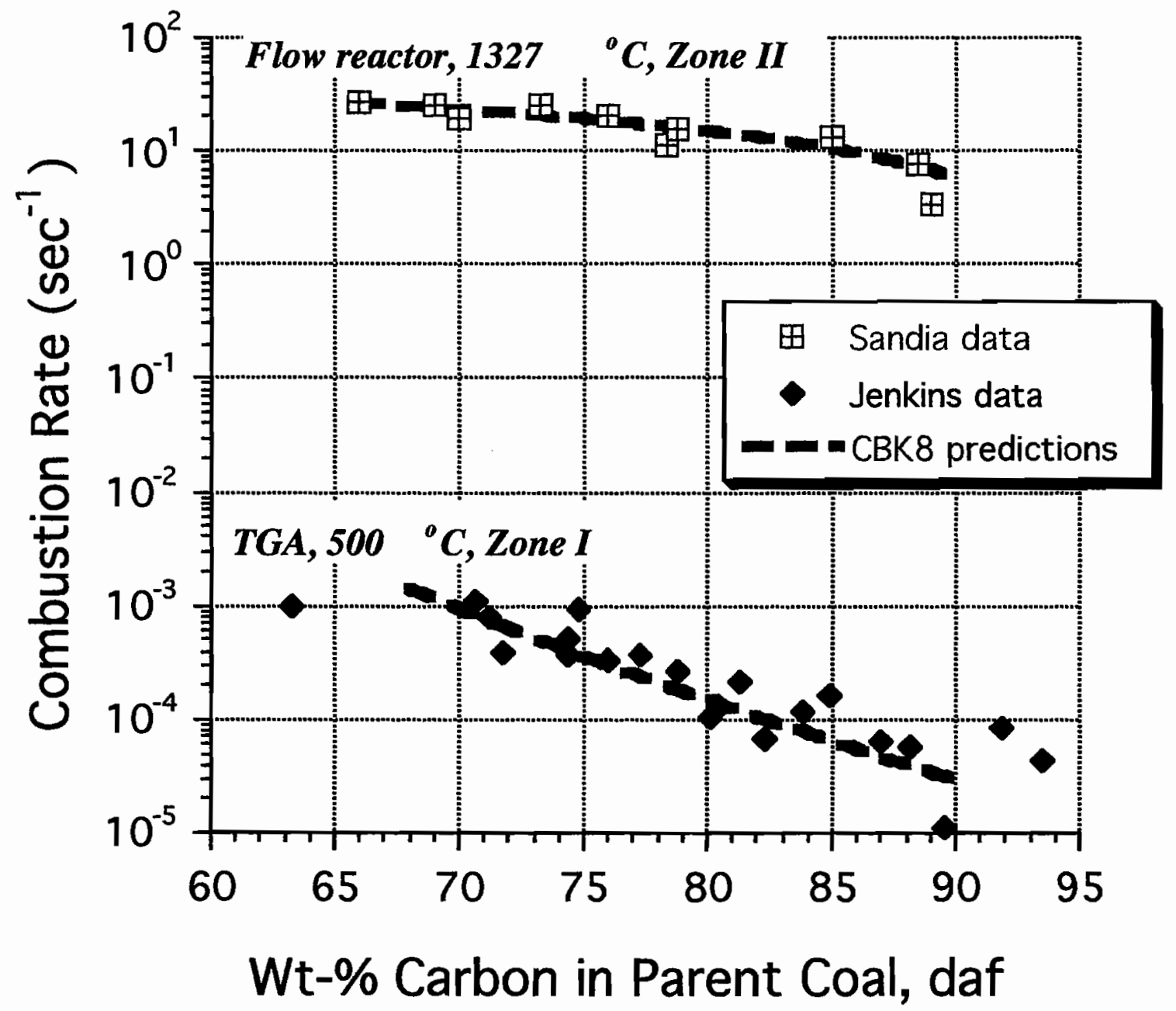

Figure 5. CBK8 predictions of absolute char reactivities and their rank dependencies under two sets of conditions. The upper points are maximum burning rates measured by sampling in the Sandia flame supported flow reactor [Hurt and Mitchell, 1992], with gas temperature $1600 \mathrm{~K}$, oxygen concentration $12 \mathrm{~mol}-\%$, nominal particle diameter $100 \mu \mathrm{m}$, using U.S. coals from lignites to low-volatile bituminous. The TGA data are from Jenkins et al. [1973] in air at $500^{\circ} \mathrm{C}$, on chars prepared by 2 -hr heat treatment in inert gas from U.S. of various rank.

\footnotetext{
$\dagger$ The internal reaction / diffusion treatment is essential for extrapolation to lower temperatures, due to the change in effective activation energy between zones I and II. The annealing model is required to translate reactivities between the different heat treatment histories in the two data sets. The TGA chars were thermally stabilized by 2-hr heat treatment at $1000{ }^{\circ} \mathrm{C}$ prior to oxidation at $500{ }^{\circ} \mathrm{C}$, while the Sandia chars were heat treated in situ at about $1750 \mathrm{~K}$ particle temperature for about 50 milliseconds.
} 
lacy ash observed in CPI experiments $(\theta=0.5)$. The intraparticle transport parameter, $\tau / f=6$ lies in the expected range, as $\tau$ values for heterogeneous catalysts typically lie between 2 to 6 [Satterfield, 1981], while $f$ must be less than unity. The unification of Zone I and Zone II data requires proper decoupling of intrinsic reaction and pore diffusion, so the successful result in Fig. 11 also suggests that $\tau / f$ has been appropriately estimated.

\section{Chapter 5 Conclusions}

1. The nonlinear equations describing heterogeneous reaction and transport predict extinction-like events during combustion of single char particles under some conditions, in agreement with data. The extinction-like events greatly increase the time required to achieve the high conversion desired in combustion practice.

2. There are multiple pseudo steady state solutions to the single particle energy balance at gas temperatures below about $1300 \mathrm{~K}$ and oxygen concentrations well above $12 \mathrm{~mol}-\%$ at atmospheric pressure. Under these conditions, decreases in gas temperature, diameter, or reactivity can eliminate the high temperature solution and cause a discontinuous jump to a much lower pseudo steady state temperature, referred to as extinction. Under conditions directly relevant to pulverized coal technologies (higher temperatures and lower oxygen concentrations) these multiple steady states do not occur. Particle temperatures decrease continuously but often significantly as burnout proceeds, a phenomenon referred to as near-extinction.

3. This work confirms the conclusions reached by Essenhigh, Förtsch, and Klimesh that a transition from Zone II to Zone I can occur under pulverized coal combustion conditions and that this transition must be described explicitly to correctly model extinction behavior.

4. The most common cause of extinction-like events under pulverized coal combustion conditions are reductions in particle diameter and gas temperature, accompanied by a transition from Zone II to Zone I burning. In high reactivity materials extinction can be delayed to very high conversion (95\%), where the primary cause is transport modification by mineral matter. Mineral matter can also cause the extinction of larger particles $(>300 \mu \mathrm{m})$ which can develop ash films while at relatively low conversions. Extinction events caused primarily by annealing are predicted to be rare.

5. An kinetic model accounting for internal reaction / diffusion, annealing, and ash effects is capable of describing a large set of data on extinction-like events. The model (CBK8) with the same parameter set also successfully describes the TGA data of Jenkins et al. at $500{ }^{\circ} \mathrm{C}$ for a wide range of U.S. coals, thus unifying reactivity measurements spanning over $1000{ }^{\circ} \mathrm{C}$ in particle temperature and $4-5$ orders of magnitude in reaction rate. 


\section{References for Chapter 5}

Carberry, J.J., A.I.Ch.E.J. 8557 (1962).

Essenhigh, R.H., in Chemistry of Coal Utilization, Second Supplementary Volume (M.A. Elliot, ed.), Wiley, New York, 1981, p. 1153.

Essenhigh, R.H., Klimesh, H.E., and Förtsch, D.: Combustion Characteristics of

Carbon: Dependence of the Zone I-Zone II Transition Temperature (Tc ) on Particle Radius; Energy and Fuels, in press (1999).

Essenhigh, R.H., Förtsch, D., and Klimesh, H.E.: Combustion Characteristics of Carbon: Influence of the Zone I-Zone II Transition of Particle Burn-Out in PC Flames; Energy and Fuels in press, (1999).

Froment, G.F., Bischoff, K.B., Chemical Reactor Analysis and Design, p. 162, John Wiley and Sons, New York, 1990.

Gavalas, G.R. A.I.Ch.E.J. 26 (4) 577 (1980).

Hurt, R. H. and R. E. Mitchell. Twenty-Fourth International Symposium on Combustion, The Combustion Institute, Pittsburgh, PA, 1992, pp. 1243 - 1250.

Hurt, R. H. and Davis, K. A., Twenty-Fifth Symposium (International) on Combustion, The Combustion Institute, Pittsburgh, 1994, pp. 561-568.

Hurt, R.H., Sun, J-K, and Lunden, M., Combustion and Flame, 113 181-197 (1998).

Hurt, R.H., Davis, K.D., Combustion and Flame, $116662-670$ (1999).

Hurt, R.H., Twenty-Seventh International Symposium on Combustion, The Combustion Institute, Pittsburgh, 1998, 2887-2904.

Lunden, M.M., Yang, N.Y.C., Headley, T.J., Shaddix, C., Twenty-Seventh Symposium (Int) on Combustion, The Combustion Institute, Pittsburgh, 1998, pp. 1695-1702.

Mitchell, R.E., Twenty-Third Symposium (International) on Combustion, The Combustion Institute, Pittsburgh, 1990, pp. 1297-1304.

Jenkins, R.G., Nandi, S.P., Walker, P.L.Jr., Fuel 52288 - 293 (1973).

Satterield, C.N., Mass Transfer in Heterogeneous Catalysis, Robert Krieger, Huntington, New York, 1981.

Simons, G.A, Prog. Energy Combust. Sci. 9 269-290 (1983). 


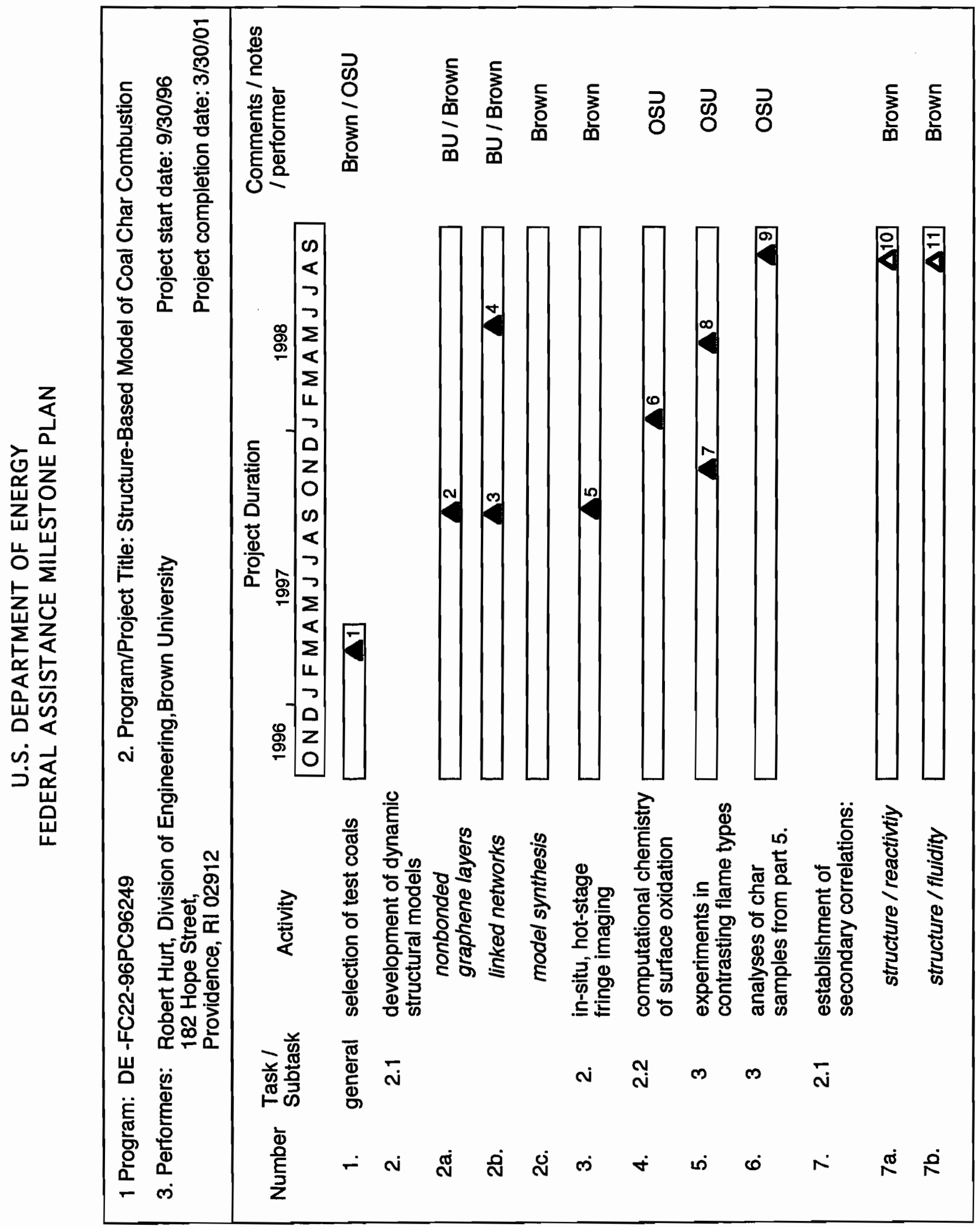




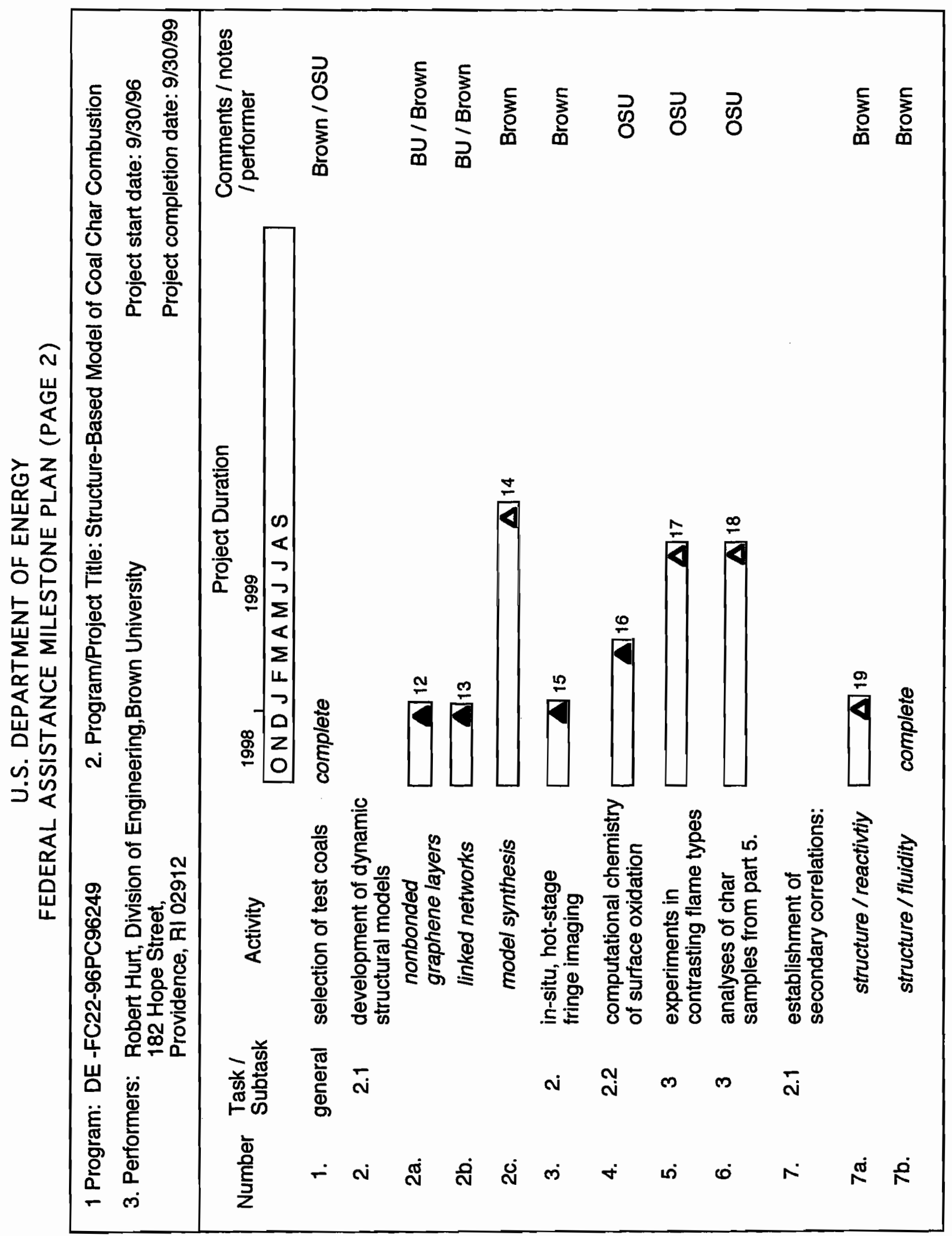




\section{U.S. Department of Energy \\ Milestone Log}

Project title: Structure-based models for char combustion

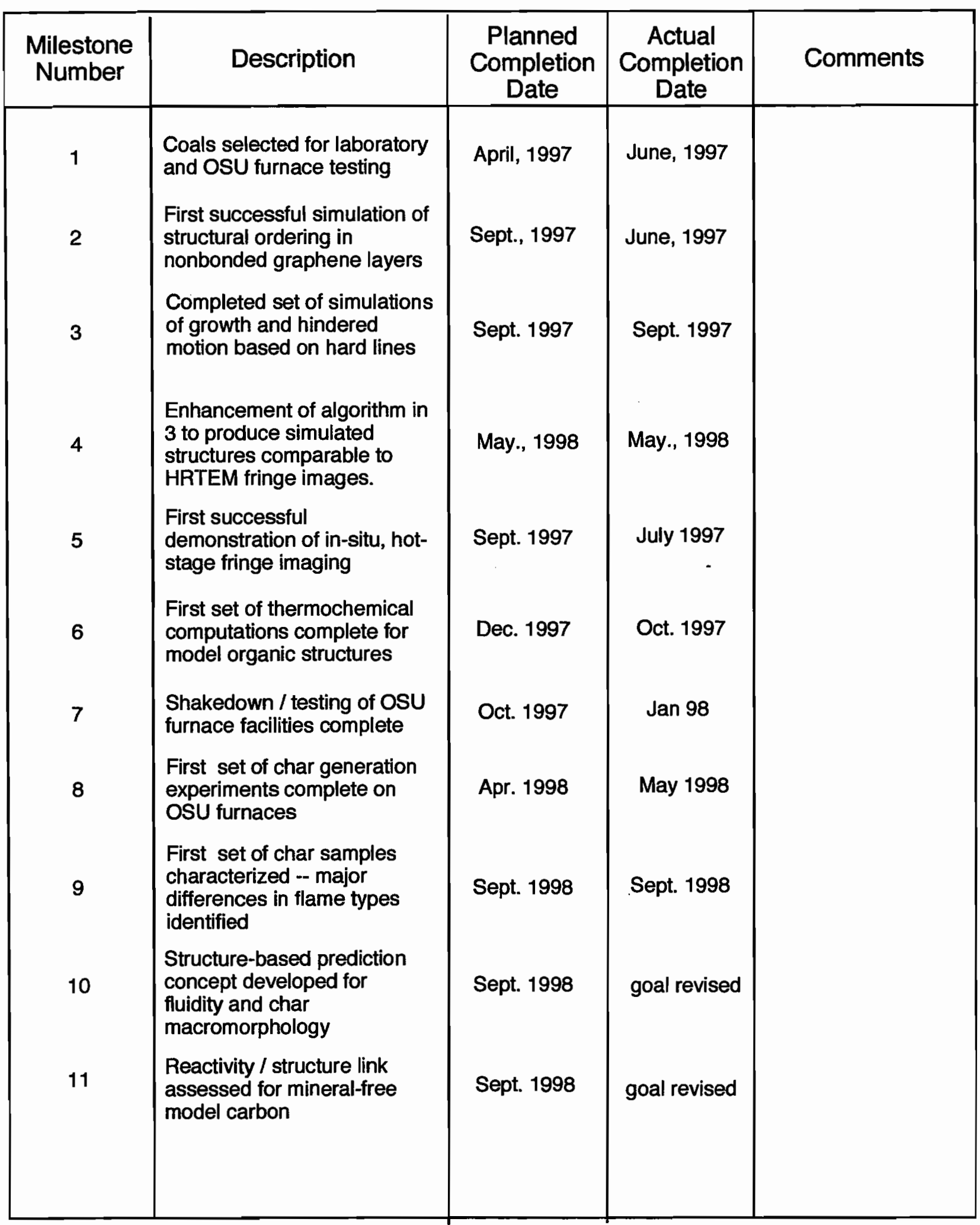




\section{U.S. Department of Energy \\ Milestone Log (page 2)}

Project title: Structure-based models for char combustion

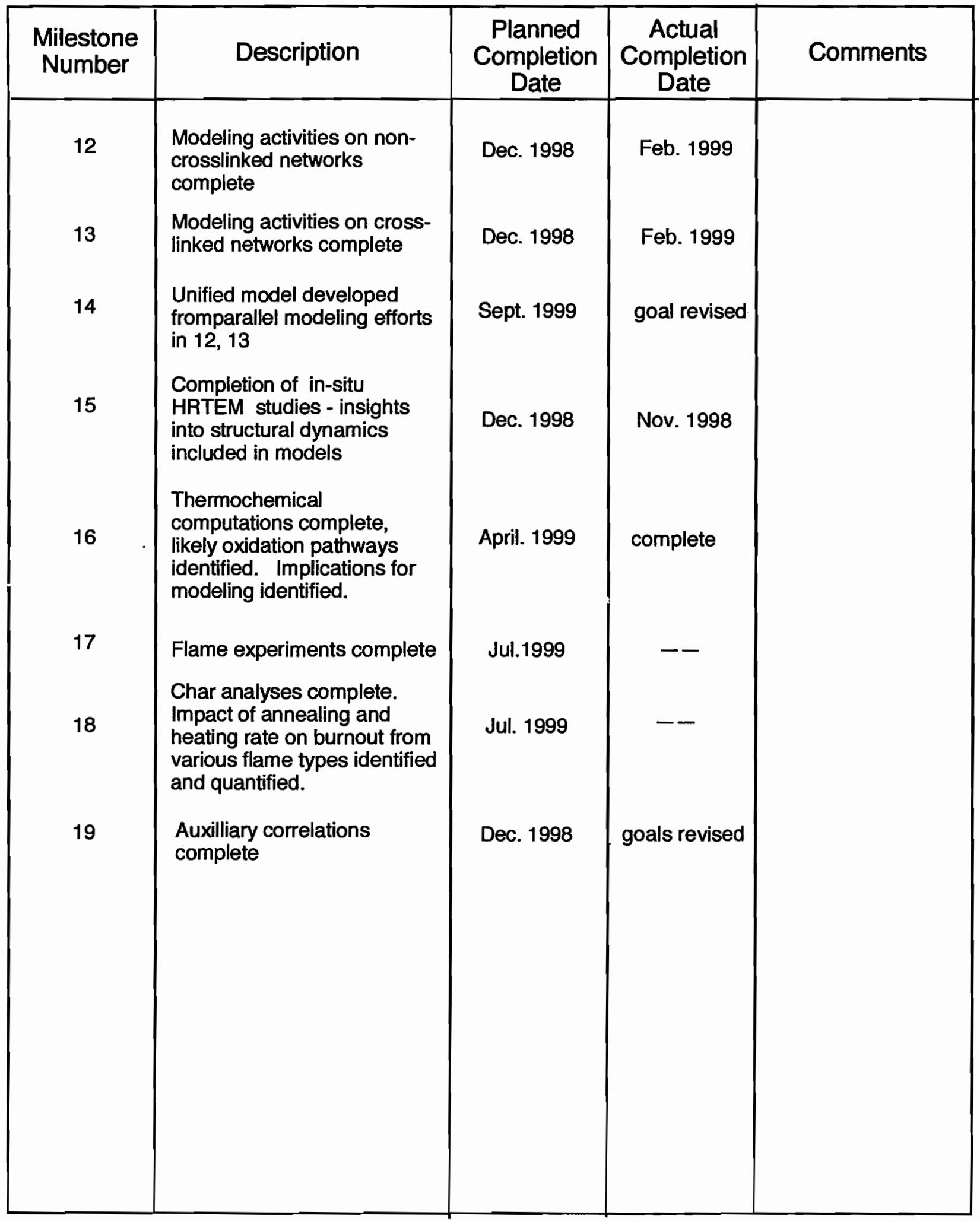

